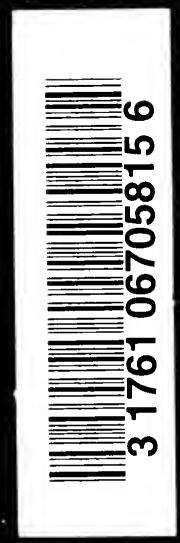




$\mathrm{OF}$

\section{GASES AND LIQUIDS}

BY

RICHARD D. KLEEMAN,

D.Sc. (Adelaide), B.A. (Cantab.)

Associate Professor of Physics, Union College; Consulting Physicist and Physical Chemist; formerly 1851 Exhibition Scholar of the

University of Adelaide, Research Student of Emmanuel

College, Cambridge, Mackinnon Student of the Royal Society of London, and Clerk Maxwell

Student of the University of Cambridge

FIRST EDITION

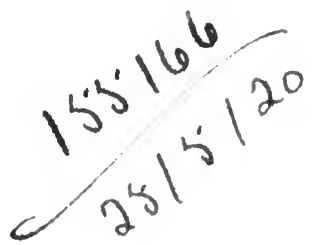

NEW YORK

JOHN WILEY \& SONS, INC.

LONDON: CHAPMAN \& HALL, LIMITED

1920 
Copyright, 1920

BY

RICHARD D. KLEEMAN 
DEDICATED

TO MY PARENTS 
Digitized by the Internet Archive in 2007 with funding from Microsoft Corporation 


\section{PREFACE}

ThE object of writing this book is to formulate a Kinetic Theory of certain properties of matter, which shall apply equally well to matter in any state. The desirability of such a development need not be emphasized. The difficulty hitherto experienced in applying the results obtained in the case of the Kinetic Theory of Gases in the well-known form to liquids and intermediary states of matter has been primarily due to the difficulty of properly interpretating molecular interaction. In the case of gases this difficulty is in most part overcome by the introduction of the assumption that a molecule consists of a perfectly elastic sphere not surrounded by any field of force. But since such a state of affairs does not exist, the results obtained in the case of gases hold only in a general way, and the numerical constants involved are therefore of an indefinite nature, while in the case of dense gases and liquids this procedure does not lead to anything that is of use in explaining the facts.

Instead of an atom, or molecule, consisting of a perfectly elastic sphere, it is more likely that each may be regarded simply as a center of forces of attraction and repulsion. If the exact nature of the field of force surrounding atoms and molecules were known, it would be a definite mathematical problem to determine the resulting properties of matter. But our knowledge in this connection is at present not sufficiently extensive to permit a development of the subject along these lines. But in whatever 
way the subject is developed fundamental progress will have been made only if molecular interaction is not, as is usually the case, represented by the collision of elastic spheres. It will be shown in this book that the subject may be developed to a considerable extent along sound mathematical lines yielding important results without knowing the exact nature and immediate result of molecular interaction. Thus it will be found, for example, that the definition of the free path of a molecule in connection with viscosity, conduction of heat, diffusion, etc., may be given a form in each case not involving the exact nature of molecular interaction, which is mathematically quite definite, and which therefore applies equally well to the liquid and gaseous states. Since in the gaseous state each kind of path is proportional to the volume of the gas, its interest is then mainly associated with the characteristic factor of the volume which makes the product numerically equal to the path. A direct physical meaning may be given to this factor.

In constructing a general Kinetic Theory the problem that presents itself first for investigation is the dependence of the velocity of translation of a molecule in a substance on its density and tempcrature. It is often assumed that this velocity is the same in the liquid as in the gaseous state at the same temperature. It can be shown, however, that this holds only for each molecule at the instant it passes through a point in the substance at which the forces of the surrounding molecules neutralize each other. The total average velocity corresponding to the whole path of a molecule is usually much greater than the foregoing velocity in a liquid and dense gas on account of the effect of the molecular forces of attraction and repulsion.

The pressure which the molecules exert tending to expand the substance, or the expansion pressure, and the number of molecules crossing an area of one square centimeter from one side to the other per second, evidently depend on the 
magnitude of the total average velocity. It can be shown that if the molecules have no apparent volume, or no volume associated with each molecule through which another molecule cannot pass, the expansion pressure is equal to the number of molecules crossing a square centimeter per second multiplied by a factor whose value is the same as if the substance were in the perfectly gaseous state at the same temperature and density. The apparent molecular volume of the molecules may be defined as the quantity whose change in magnitude at constant temperature and density would not affect the total average velocity. Its definition is thus mathematically definite. The effect of this quantity on the expansion pressure is to increase it in the ratio of the volume of the substance to the difference between the volume and the apparent molecular volume. The expansion pressure of a substance is evidently balanced by the sum of the external pressure and the intrinsic or negative pressure due to the attraction of the molecules upon each other, the equation expressing this relationship being a form of the equation of state.

In the case of a mixture it is convenient to introduce the terms " partial expansion pressure," "partial intrinsic pressure," and "partial external pressure," in connection with each constituent. Each partial expansion pressure is balanced by the sum of the corresponding partial intrinsic and partial external pressures, similarly as in the case of a pure substance. The property of osmotic pressure can then be shown to be a function of the corresponding partial intrinsic pressure of the mixture, and the number of molecules giving rise to the osmotic pressure which cross a square centimeter from one side to the other per second. This is what we would expect, since osmotic pressure must be the outcome of the fundamental properties of a substance-the motion of translation of its molecules, and the forces they exert upon each other. 
With the foregoing results as a basis, and the modified definitions of the free paths of a molecule mentioned, the foundation of a general Kinetic Theory can be laid which applies to matter in any state, and which furnishes a number of important formulæ. These formulæ may be given important extended forms containing quantities which are arbitrary in so far as they satisfy the formulæ as a whole. Formulæ may also be deduced along the same lines involving instead of the molecular free path the projection of the motion of a molecule along a line. This projection and its period are also arbitrary in so far as they satisfy the formulæ as a whole. Some of them find an interesting application in connection with colloidal solutions. The foundation of the subject may be said to be fairly complete, since it furnishes the structure about which further advances may be made so that the subject can be rendered more or less complete. These advances will consist largely in expressing the constants involved in the fundamental formulæ in terms of the constants of the molecular forces and the molecular volume. If the formulæ obtained in connection with viscosity, conduction of heat, and diffusion, be applied to a perfect gas, they assume the well-known forms, but the symbols have somewhat different meanings.

The development presented is perfectly sound without involving difficult mathematics. This has, in fact, been one of the main objects kept in view. As a physicist I have often failed to see the usefulness from a physical standpoint of the extremely intricate mathematical investigations purporting to work out to the utmost limit the results of certain assumptions (usually in connection with molecular collision), and which have usually led to results whose usefulness seems incommensurate with the labor involved, seeing that the assumptions are usually not likely to be true. It is desirable that there should be a simple and clearcut connection between 
theory and experiment, and the physical side of the subject has therefore been kept in prominence.

The development of a general Kinetic Theory of matter will be of service in the study of chemical action, principally in connection with the constant of mass-action and the reaction velocity constants. On the whole the kinetic aspect of the chemical interaction of molecules apart from the thermodynamical aspect, which does not take into account the individual nature of the different effects producing a resultant whole, can only proceed along lines having such a Kinetic Theory as a basis. Thus it will readily be seen that the rapidity of a chemical reaction in a gaseous or liquid mixture must be intimately connected with the number of molecules crossing a square centimeter per second in the case of each constituent. The constant of mass-action must evidently be intimately connected with the free diffusion path of a molecule, etc.

The study of viscosity, conduction of heat, diffusion, etc., has usually been confined to pure substances and to mixtures whose constituents do not interact chemically. It would evidently be of great interest to study these effects in the case of substances partly dissociated, since the interacting between molecules is then influenced by chemical affinity. Further light may be thrown on the nature of this property by the application of the formulæ obtained.

I have previously published some investigations in various scientific journals along the lines pointed out. Since the development of a subject can only be gradual, it was necessary to modify some of these results before incorporating them into this book. The other investigations in the book which more or less complete the Kinetic Theory along the lines mentioned I have not published previously. It seemed that it would be better to present the subject as a whole to the scientific public, since the various results are 
intimately connected and could therefore not be presented in detached parts without a good deal of reference to, and recapitulation of, preceding parts being necessary, to the inconvenience of the reader.

The book has also been brought up to date in matters not connected with molecular collision, and has been treated in a way so that the results are connected as directly as possible with the results of experiment.

The properties of matter treated in this book may be said to depend mainly on the dynamical properties of molecules modified in most cases by the molecular forces of attraction and repulsion. There is evidently, therefore, another side to the subject of the properties of matter, namely that which deals with those properties which depend in the main on the molecular forces modified in some cases by the dynamical properties of the molecules. Thus, for example, the internal heat of evaporation and the intrinsic pressure probably do not depend directly on molecular motion. This part of the physico-chemical properties of matter will be dealt with in a separate book under the title "Molecular Forces." The present book may serve together with the foregoing as an introduction to the study of the purely thermodynamical aspect of material properties.

Union College, Schenectady.

R. D. KleEman.

March, 1919. 


\section{CONTENTS}

\section{CHAPTER I}

The Molecular Constants, and the Dynamical Properties of a Molecule in the Gaseous State

8EC.

1. A Brief Historical Summary of the Development of the Kinetic Theory of Explaining the External Pressure and other Properties

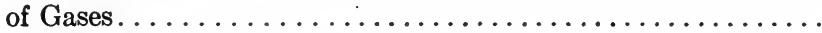

2. A Direct Experimental Proof that Matter consists of a large number of Entities or Atoms.................. 3

3. The Absolute Mass of an Atom determined from a Knowledge of the Electric Charge $e$ carried by an Electron.

PAGE

4. Indirect Experimental Evidence that the Molecules of Gases and Liquids are in Rapid Motion................. 8

5. The Absolute Temperature; and the Equation of a Perfect Gas, and of a Mixture of Gases

6. The Velocity of Translation of a Molecule in a Gas from Dynamics.

7. Maxwell's Law of Distribution of the Velocities of the Molecules of a Gas.

8. The Average Kinetic Energy Velocity of a Molecule, and the Relation between the Kinetic Energy of a Molecule and its Absolute Temperature..........................

9. The Equipartition of Kinetic Energy between the Different Molecules in a Mixture of Gases. . . . . . . . . . . . . . 31

10. The Number of Molecules per Cubic Cm. in a Gas.........

11. The Number of Molecules Crossing a Square Cm. in all Directions from one Side to the Other in a Gas. . . . . . . . . 34

12. The First Law of Thermodynamics.............. 37

13. The Specific Heats of Gases and Liquids............. 39

14. Evidence that Molecules and Atoms are surrounded by Fields

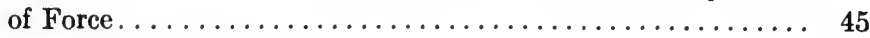

15. Molecular Interaction .................... 48 


\section{CHAPTER II}

The Effect of the Molecular Forces on the Drnamical Properties of a Molecule in a Dense Gas or Liquid

16. The Velocity of Translation of a Molecule in a Liquid or Dense Gas when passing through a Point at which the Forces of the Surrounding Molecules neutralize each other....... 51

17. The Total Average Velocity of Translation of a Molecule in a Substance.

18. The Number of Molecules crossing a Square Cm. in a Substance of any Density from one Side to the Other per Second. .

19. The Effect of Molecular Volume on $p$ and $n$ in the Case of an

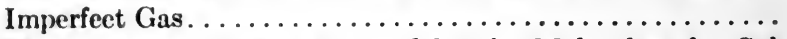

20. The Expansion Pressure exerted by the Molecules of a Substance of any Density.

21. The Intrinsic Pressure; and the Equation of Equilibrium of a Substance.......................... 69

22. Superior and Inferior Limits of $n$ of a Substance....... 74

23. Superior and Inferior Limits of $V_{t}$ of a Substance....... 75

24. The Real and Apparent Volumes of a Molecule, and their Superior Limits. ...................... 77

25. Inferior Limits of $n$ and $V_{t} \ldots \ldots \ldots \ldots \ldots \ldots \ldots \ldots \ldots \ldots$

26. The Equation of State of a Substance............. 81

27. The Conditions that the Equation of State has to satisfy .... 86

28. The Relation of Corresponding States............. 91

29. The Determination of the Quantities $n, V_{l}$, and $b$, of a Substance.............................. 95

30. An Equation Connecting the Intrinsic Pressure, Specific Heat, and other Quantities. . . . . . . . . . . . . . . 103

31. The Mean Free Path of a Molecule under Given Conditions. . 106

\section{CHAPTER III}

\section{Quantities which Depend Directly on the Nature of Molecular Motion}

32. The Coefficient of Viscosity of a Substance........... 110

33. The Viscosity Mean Momentum Transfer Distance of a Molecule in a Substance....................... 113

34. Formula for the Viscosity in Terms of Other Quantities..... 116

35. Formula for the Viscosity of Mixtures................ 142 
36. The Coefficient of Conduction of Heat. . . . . . . . . 147

37. The Mean Heat Transfer Distance of a Molecule in a Substance.............................. 148

38. Formulæ for the Coefficient of Conduction of Heat in Terms of Other Quantities....................... 150

39. Formulæ for the Coefficient of Conduction of Heat of a Mixture of Substances......................... 103

40. The Coefficient of Diffusion of a Substance............ 168

41. The Mean Diffusion Path of a Molecule in a Mixture....... 169

42. Formulæ Expressing the Coefficient of Diffusion in Terms of Other Quantities........................ 170

43. Maxwell's Expression for the Coefficient of Diffusion of Gases 187

\section{CHAPTER IV}

Miscellankous Applications, Connections, and Extensions of the Results of the Previous Chapters

44. The Direct Observation of some of the Quantities depending on the Nature of the Motion of a Molecule in a Substance, and their Use.......................... 189

45. The Coefficient of Diffusion in Connection with Osmotic Pressure and the Coefficient of Mobility . . . . . . . . 196

46. Partial Intrinsic Pressures.................... 202

47. Conditions of the Equilibrium of a Heterogeneous Mixture such as Two Phases in Contact. . . . . . . . . . . . 206

48. Osmotic Pressure expressed in Terms of the Kinetic Properties of Molecules. . . . . . .................. 208

49. The Velocity of Translation of Particles undergoing Brownian Motion. . . . . . ..................... 210

50. The Osmotic Pressure of a Dilute Solution of Molecules and their Velocity of Translation.................. 213

51. A Direct Determination of $N$, the Number of Molecules in a Gram Molecule........................ 217

52. Formulæ involving Stokes' Law . . . . . . . . . . . . 220

53. Extended Forms of the Diffusion Equations.......... 222

54. Extended Forms of the Viscosity Equations............ 230

55. Extended Forms of the Heat Conduction Equations........ 238

56. The Constant Period Displacement Diffusion Equation and

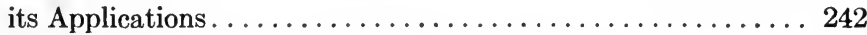

57. The Constant Displacement Diffusion Equation ........ 252 
sxc.

58. The Constant Period and Constant Displacement Viscosity

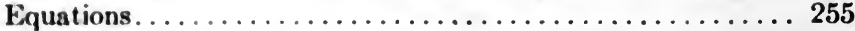

59. The Constant Period and Constant Displacement Heat Conduction Equations......................... 259

60. Another Method of Determining the Total Average Velocity of Translation of a Colloidal Particle............... 262

61. The Distribution of the Molecular Velocities in a Substance not Obeying the Gas Laws.................... 265 


\section{NOTE}

IT will be useful to give a list of the most important symbols used in a general way in this book, the kind of molecule and phase to which a symbol refers in any given case being indicated by the addition of suffixes and dashes when necessary.

$p=$ external pressure

$P_{n}=$ intrinsic pressure

$P_{s}=$ asmotic pressure

$v=$ volume of a gram molecule of a substance

$\vartheta=$ external molecular volume connected with the concentration changes of a mixture

$b=$ apparent internal molecular volume connected with molecular motion

$\rho=$ density

$T=$ absolute temperature

$S=$ specific heat

$n=$ number of molecules crossing a square $\mathrm{cm}$. from one side to the other per second

$m=$ relative molecular weight

$m_{a}=$ absolute molecular weight

$N=$ number of molecules in a gram molecule

$N$ with suffixes $=$ molecular concentration

$V=$ average kinetic energy velocity of a molecule in the gaseous state

$V_{a}=$ average velocity of a molecule in the gaseous state

$V_{l}=$ total average velocity of a molecule in any state

$\eta=$ coefficient of viscosity 
$C=$ coefficient of conduction of heat

$D=$ coefficient of diffusion

$\delta=$ rate of diffusion

$l_{\text {, }}=$ mean momentum transfer distance

$l_{c}=$ mean heat transfer distance

$l_{\mathrm{s}}=$ mean diffusion path

$\Phi=$ interference function

$\kappa=$ path factor

$d=$ projection of the motion of a molecule, or its displacement

$t=$ period of displacement. 


\section{A KINETIC THEORY \\ OF \\ GASES AND LIQUIDS}

\section{CHAPTER I}

THE MOLECULAR CONSTANTS, AND THE DYNAMICAL PROPERTIES OF A MOLECULE IN THE GASEOUS STATE

1. A Brief Historical Summary of the Development of the Kinetic Theory of Explaining the External Pressure and other Properties of Gases.

In order to explain the external pressure, or elasticity of a gas, vapor, or liquid, it is necessary to introduce a theory, since the mechanism giving rise to this property is not evident to the eye. A purely mechanical explanation based on the observed elasticity of solid materials, or of a mass of fibrous matter, no doubt presented itself to the minds of the early physicists. But since from the earliest times some philosophers regarded matter as consisting of a number of hard, indivisible, and similar parts, it was natural that it should occur to some to explain the elasticity of a gas by the motion and consequent change of momentum of these parts. Thus Gassendi in the 17th century elaborated an atomic theory of the properties of matter based upon the assump- 
tion that all the material phenomena can be referred to the indestructible motion of atoms. He supposed that all atoms are the same in substance, but different in size and form, and that they move in all directions through space. A number of processes, in particular the transition of matter from one state to the other, were explained on this basis. Later these ideas occurred independently to other investigators, who elaborated them to a greater extent. Thus Daniel Bernoulli in his Hydrodynamica, published in 1738 , pointed out that the elasticity of a gas may be explained by the impact of the particles of which it was supposed to consist on the walls of the containing vessel, and accordingly he deduced Boyle's law for the relation between pressure and volume. Later the subject was taken up by Herapath, Watertson, Joule, Krönig, and with great success by Clausius. Some time later Maxwell added some important contributions to the Kinetic Theory. In the hands of Clausius and Maxwell it developed with great rapidity and success. The subject now attracted numbers of theoretical and experimental investigators who helped to perfect it theoretically, and by testing the results experimentally demonstrated the soundness of the underlying assumptions. Of the theoretical investigators Boltzmann should be specially mentioned.

The endeavor by scientists in recent years to extend the well-known mathematical investigations of the Kinetic Theory of Gases to liquids and dense gases, supposing that molecular interaction may be represented by the collision of elastic spheres, has been almost barren of results. The object of this book, as has already been pointed out in the Preface, is to give the Theory such a form that these diffculties are removed, and that it accordingly applies as conveniently to liquids as to gases. Van der Waals has already rendered important service by means of his theory of continuity of state, in indicating the general nature of the relation between the pressure, volume, and temperature of a sub- 
stance in any state, and of two states in equilibrium with each other.

In this development a knowledge of the nature of the relative distribution of matter in space is of foremost importance, and therefore claims first attention.

\section{A Direct Experimental Proof that Matter} consists of a large Number of Entities or Atoms.

This was first furnished definitely by an experiment devised by Rutherford and Geiger.* It was arranged that $\alpha$ particles of radium were fired through a gas at low pressure, exposed to an electric field. This gave rise to ionization in the gas which was measured in the usual way. The amount of ionization obtained was considerably increased by increasing the field to near sparking value. The velocity given to the initial ions was then so large that they produced further ions by collision with neutral molecules. In this way the small ionization produced by one $\alpha$ particle in passing through the gas could be magnified several thousand times. The sudden current through the gas, due to the entrance of a single $\alpha$ particle in the detecting vessel, was by this method increased sufficiently to give an easily measurable deflection to the needle of an ordinary electrometer. Thus by limiting the number of $\alpha$ particles shot into the vessel by means of a stop, a succession of throws of the gal-

* Proc. Roy. Soc. A. 81, p. 141 (1908); Phys. Zeit. 10, p. 1 (1909).

Note. There existed previously a good deal of indirect evidence that matter consists of entities. Thus the law of constant proportion, and others, in chemistry could very simply be explained if this were the case. Also the formulæ obtained for the viscosity, conduction of heat, of a gas, deduced from the assumption that it consists of particles of matter in motion, gave a general agreement with the facts. But each of these results might also hold on mathematical grounds without matter necessarily consisting of atoms or molecules. A definite proof of the atomistic nature of matter was therefore desirable and of importance, and this was first furnished by the experiments quoted. 
vanometer needle was obtained. This proved the atomistic nature of the $\alpha$ radiation given off by radium.

Ramsay and Soddy* had previously shown that helium was produced from radium emanation, and that it is therefore one of the products of the disintegration of radium. It was suspected that helium might consist of $\alpha$ particles which have lost their electric charge. This was proved hy Rutherford and Royds $\dagger$ who showed that accumulated $\alpha$ particles, quite independently of the matter from which they were expelled, consist of helium. The radioactive material was enclosed in a glass tube whose walls were so thin that they were penetrated by the expelled $\alpha$ particles, which were retained by a vessel containing the glass tube. The matter collected in this way was tested spectroscopically and otherwise, and found to consist of the gas helium. The atomistic nature of one of the gases was thus proved; and since different gases possess similar physical properties this result is likely to hold for all of them.

Some interesting experiments by Dunoyer $\ddagger$ may be mentioned which can only be reasonably explained if matter consists of entities which possess motion of translation. A cylindrical tube was divided into three compartments by means of two partitions perpendicular to the axis of the tube, each partition being pierced centrally by a small hole so as to form a diaphragm. The tube was fixed with the axis vertical and a piece of some substance such as sodium, which is solid at ordinary temperatures, placed at the bottom of the lowest compartment. The tube was now exhausted and the substance heated to a sufficient temperature to vaporize it. The vaporized particles were projected in all

-Nature, 68, p. 246 (1903); Proc. Roy. Soc., A. 72, p. 204 (1903); • 73. p. 316 (1904).

† Phil. Mag., 17, p. 281 (1909). 142.

† Compies Rendus, 152 (1911), p. 592; Le Radium, VIII (1911), p. 
directions, some of which passed through the hole of the first diaphragm at various angles. Of these some passed through the hole in the second diaphragm forming a deposit on the top of the tube. This deposit was found to coincide exactly with the projection of the hole in the second diaphragm formed by radii drawn from the hole in the first diaphragm. A small obstacle placed in the path of the particles was found to form a shadow on the upper surface of the tube. The vaporized matter thus moved along straight lines, and therefore could conceivably consist only of particles, i.e., atoms.

3. The Absolute. Mass of an Atom is Most Accurately Determined from a Knowledge of the Electric Charge e Carried by an Electron.

For this reason, and that the accurate determination of the value of $e$ is important in itself, a number of different principles and methods have been employed in recent years to determine this quantity with the greatest possible accuracy.

A determination of $e$ from radioactive data was made by Rutherford and Geiger.* The number of $\alpha$ particles passing into a vessel of the kind mentioned in the previous Section were directly counted, and also the total electrical charge carried by the particles determined. It was thus found that each particle carried a charge $9.3 \times 10^{-10}$ units, and from various evidence it was concluded that this was twice the unit charge. Similar observations were carried out by Regener, $\uparrow$ who counted the particles by noting the scintillations that they produced on impinging on a diamond. The value found by him was $e=4.79 \times 10^{-10}$.

Millikan $\ddagger$ perfected a method in which minute drops of

* Proc. Roy. Soc., A 81, p. 162 (1908).

$\dagger$ Ber. d. K. Preuss. Akad. d. Wiss., 38, p. 948 (1909).

$\ddagger$ Phil. Mag., 19, p. 209 (1910); Science, 32, p. 436 (1910). 
a non-volatile liquid are introduced between two parallel and horizontal plates having a strong electric field between them. The drops were obtained by spraying and became electrically charged during the process. The movements of individual drops under the action of gravity and the electric field were observed by means of a microscope. By adjusting the electric field to counteract gravity, it was found possible to keep a charged drop suspended in nearly the same position in the gas for an hour at a time. In that case if $X$ denotes the electric field, $N^{\prime} e$, the charge on a drop

$$
X N^{\prime} e=\frac{4}{3} g \rho r^{3},
$$

where $r$ denotes the radius of the drop, $g$ the gravitational constant, and $\rho$ the density of the material. The value of $r$ was obtained from observations of the rate of fall of the drop under the action of gravity. According to Stokes' equation the rate of fall $V_{s}$ is given by

$$
V_{s}=\frac{2 g \rho r^{2}}{9 \eta}, \quad . . . . .
$$

where $\eta$ denotes the coefficient of viscosity of the gas through which the drop moves. A number of experiments were made to test the validity of Stokes' equation for drops of different sizes, and a suitable correction was made from the observations. The value of $N^{\prime} e$ accordingly could be determined. The experiments showed that each of these drops carried a definite multiple of a small charge, which accordingly is equal to $e$. The final result obtained gave a value* $e=4.77$ $\times 10^{-10}$ E. S. U.

If $e$ be the charge of electricity carried by the hydrogen atom in electrolysis, and $N$ the number of atoms in one

- The Electron, by R. A. Millikan. The University of Chicago Science Series. 
gram molecule of hydrogen, it is known from experiments on the electrolysis of solutions that

$$
N e=9647 \text { E.M.U. }
$$

Now according to Rutherford and Geiger

or

$$
\begin{aligned}
& e=4.65 \times 10^{-10} \text { E.S.U., } \\
& e=1.55 \times 10^{-20} \text { E.M.U., }
\end{aligned}
$$

and accordingly

$$
N=6.2 \times 10^{23},
$$

which is also the number of molecules in a gram molecule of any substance, according to the definition of a gram molecule. The absolute mass $m_{a H}$ of a hydrogen atom is equal to $1 / N$, and hence

$$
m_{a H}=1.60 \times 10^{-24} \mathrm{grm} .
$$

The number of molecules in a cubic $\mathrm{cm}$. of any gas at standard pressure and temperature can now be shown simply from density considerations to be equal to $2.78 \times 10^{19}$. Millikan's value of $e$ gives

$$
N=6.06 \times 10^{23},
$$

and

$$
m_{a H}=1.65 \times 10^{-24} \mathrm{grm} .
$$

The remarkable result that the number of molecules in a gas under standard conditions is a contant independent of the nature of the gas, and the fact that a gas as a whole is compressible, suggests that the molecules are not packed closely together, but separated from each other by distances much greater than their own dimensions, and that they undergo motion of translation so as to produce on the average an even distribution of matter in space, and give rise to the external pressure of the gas. 


\section{Indirect Experimental Evidence that the Mole-} cules of Gases and Liquids are in Rapid Motion.

It will first be shown that there are definite theoretical reasons that the molecules should be in motion.

Two ways only suggest themselves of explaining the pressure exerted by a gas, viz.: (a) By forces of repulsion between the molecules, $(b)$ by a molecular motion of translation. It can be easily shown that the pressure cannot be exerted according to $(a)$. The Joule-Thomson effect described in Section 14 indicates that forces of xttraction exist between molecules which act over distances of the order of the distances of separation of the molecules of a gas. The positive sign of the internal heat of evaporation of a liquid indicates that this also holds for much smaller distances of separation of the molecules. The pressure exerted by a gas cannot therefore be produced by forces of repulsion; and the molecules must therefore possess motion of translation to account for the pressure.

It will be shown in Section 6 from dynamical considerations that the magnitude of this motion decreases as the mass of the molecule increases. Accordingly if the molecules were sufficiently large to be visible to the eye, we might expect that this motion could actually be observed. It is evident that the motion will not be along a continuous straight line, but zig-zag shaped, since the molecules will collide with each other. This will also be the case with a particle, or a conglomeration of thousands of atoms, contained in a liquid or gas, for the particle would be struck on account of its size by a large number of molecules at the same instant, but the same number would not necessarily strike it on cach of two opposite sides, and thus the motion of the particle would be rendered undulatory in character. These conclusions are borne out in a striking way by experiment. 
On observing by means of a microscope small particles of matter suspended in a liquid, they are found to be undergoing rapid oscillatory motion. This was first studied by Brown in 1827 in the case of pollen of plants suspended in water, and hence has been called Brownian motion. The motion is best observed by means of the ultra-microscope introduced by Siedentopf and Zsigmondy.* In this apparatus a parallel beam of sunlight, or arc-light, is passed through the liquid under investigation at right angles to the axis of the microscope. If the liquid is completely homogeneous no scattered light enters the microscope. On the other hand if the liquid contains particles of matter these appear, through scattering some of the light passing through the liquid, as bright specks of light against a dark background. These specks of light undergo a rapid oscillatory motion indicating the motion of the particles. Through the contrast of the light and darkness a particle becomes much more conspicuous in this arrangement than under ordinary conditions, though a "proper view" of the particle is entirely lost. It is necessary that the incident light be as intense as possible, since the amount of scattered light increases in proportion. Also the layer of liquid should be as thin as possible in order to decrease the absorption of the scattered light, and minimize the overlapping of the scattered light from different particles.

The amplitude of the oscillation of a particle was found to depend upon its size. For a diameter of $1 \mu$, or $.001 \mathrm{~mm}$., of the particle, the amplitude is about equal to $1 \mu$, while for a diameter of $3 \mu$ the amplitude is practically zero. Particles $10-40 \mu \mu$ in diameter have amplitudes up to $20 \mu$. It appears from a discussion $\dagger$ of the ultra-microscope that it is still possible to detect by this method a linear magnitude of $6 \times 10^{-7} \mathrm{~cm} .=6 \mu \mu$, or about $1 / 100$ of a light wave.

* Drud. Ann., 10, p. 1 (1903).

† loco. cit., p. 14 (1903). 
Brownian motion has also been observed in the case of smoke particles in air.

Other designs of the ultra-microscope have been introduced besides the foregoing, of which that by Cotton and Monton* deserves special mention. In this arrangement the beam of light is passed through the liquid in such a manner that it is totally reflected from a cover glass placed on top of the liquid.

Liquids containing small particles in suspension may be prepared in various ways: by means of chemical reactions involving precipitation, etc.; by sparking different metal electrodes in a liquid, etc.

Some well-known experiments by Crookes also show that motion is associated with matter, and that an increase in temperature gives rise to an increase in this motion. Thus if a thin vane of light material is suspended vertically in a tube partly exhausted and heat radiation is allowed to fall upon it at right angles, it is deflected in the direction of propagation of heat. This effect is strikingly shown by an apparatus consisting of a number of vanes mounted on a horizontal frame which can rotate round a vertical axis in a partly exhausted tube, the planes of the vanes pass through the axis of rotation and each alternate side is covered with lampblack so as to make one side of each vane a better absorber of heat than the other side. This apparatus is known as Crookes' radiometer. An observer in the same horizontal plane as the frame carrying the vanes is therefore faced by blackened vanes on one side of the axis of rotation and by unblackened vanes on the other side of the axis. If heat radiation from a source in this plane is allowed to fall on the arrangement it rotates rapidly in a direction as if a greater pressure were applied to the blackened surfaces than to the unblackened ones. This is caused by the temperature of the vanes being raised above that of the gas through the

* Comptes Rendus, 136, p. 1657 (1903). 
absorption of heat radiation, and hence heat being conveyed from the vanes to the gas by conduction. Therefore if heat consists of kinetic energy, motion is given to the gas in the immediate neighborhood of the vanes, which by reaction gives rise to pressures acting upon them. Now the absorption of heat by the blackened sides of the vanes is greater than by the unblackened sides, and hence the rise in temperature is greater in the former case, giving rise to a greater transfer of kinetic energy to the gas, which results in a greater pressure on the blackened sides than on the unblackened ones. Therefore since the surface of a vane rapidly loses the absorbed heat when not exposed to the source a continual motion of the system results.

Further information as a guide to the development of a kinetic theory of matter is obtained from a consideration of the physical properties of a gas as a whole, which will be described in the next Section.

5. The Absolute Temperature; and the Equation of a Perfect Gas, and of a Mixture of Geses.

Experiments on the coefficient of expansion of a gas at constant pressure have shown that it is practically independent of the pressure, the temperature, and the nature of the gas, and is approximately equal to .0036625 . Therefore if $v_{0}$ and $v$ denote the initial and final volume of a gas when its temperature is changed by $t^{\circ}$, we have

$$
v=v_{0}\left(1+.0036625 t^{\circ}\right) \text {. }
$$

If the volume of the gas is kept constant and the coefficient of increase of pressure is measured, it is found to be independent of the volume, temperature, and nature of the gas, and practically the same as the foregoing coefficient, and thus

$$
p=p_{0}\left(1+.0036625 t^{\circ}\right), . \quad . \quad . \quad .
$$


where $p$ and $p_{0}$ denote the final and initial pressures respectively when the temperature is changed by $t^{\circ}$.

If we consider a mass of gas whose pressure is $p_{0}$ at the temperature $0^{\circ} \mathrm{C}$., and imagine the volume kept constant while the temperature is lowered to $-t^{\circ}$, the pressure $p$ will be given by

$$
p=p_{0}\left(1-.0036625 t^{\circ}\right) .
$$

If the cooling is continued to a temperature of $-1 / .0036625$ or $-273^{\circ} \mathrm{C}$., then

$$
p=p_{0}(1-1)=0,
$$

i.e. the gas would exert no pressure on the walls of the containing vessel at this temperature. According to the previous Section this can only occur when the velocity of translation of the molecules is zero. The temperature $-273^{\circ} \mathrm{C}$. has accordingly been called the absolute zero. The temperature of a substance may be measured from this zero and denoted by $T$, in which case $0^{\circ} \mathrm{C}$. corresponds to $273^{\circ} \mathrm{C}$., and in general $T^{\circ}=273+t^{\circ}$. The foregoing equations then give

and

$$
v \propto T,
$$

$$
p \propto T,
$$

while the experiments on the relation between $p$ and $v$ at constant temperature give

$$
v \propto \frac{1}{p},
$$

which is Boyle's law. Accordingly

$$
p v \propto T,
$$

or

$$
p v=R_{1} T,
$$

where $R_{1}$ is a constant. If $v$ refers to a mass of $M$ grams of gas we may write $R_{1}=R_{2} M$; experiment then shows that 
$R_{2}$ for different gases varies inversely as $m$ the molecular weight in terms of that of the hydrogen atom. Hence finally the equation of a gas becomes

$$
p v=\frac{M R T}{m}, . \quad . \quad . \quad . \quad . .
$$

where $R$ is an absolute constant, and $v$ refers to the volume of a gas of mass $M$. If $\rho$ denote the density of the gas it follows that $v \rho=M$. The foregoing equation may also be written

$$
p=\frac{\rho}{m_{a}} \frac{R T}{\frac{m}{m_{a}}}=N_{c} \frac{R T}{N}, . . . .
$$

where $N$ denotes the number of molecules in a gram molecule, $N_{c}$ the molecular concentration, and $m_{a}$ the absolute molecular weight of a molecule.

In the case of a mixture of molecules $e$ and $r$ the total pressure $p$ according to equation (5) is given by

$$
p=p_{e}+p_{r}=N_{e} \frac{R T}{N}+N_{r} \frac{R T}{N}=N_{e r} \frac{R T}{N},
$$

where $p_{e}$ and $p_{r}$ denote the partial pressures, $N_{e}$ and $N_{r}$ the partial concentrations, and $N_{e r}$ the total concentration of the molecules. This equation is the same in form as equation (5), which therefore also holds for a mixture of any number of constituents.

The value of $R$ may be determined by means of the foregoing equation on substituting the values of $p, v$, and $T$, of a given gas. Since, however, in practice a gas does not obey strictly equations (2) and (3), the value of the absolute zero of temperature deduced from them in terms of any arbitrary scale of temperature requires correction. The thermodynamical equation (47) which involves the absolute 
temperature is usually used for this purpose. If $t$ denote the temperature readings of any arbitrary thermometer the equation may be written

$$
\left(\frac{\delta U}{\delta v}\right)_{t}=T\left(\frac{\delta p}{\delta t}\right)_{0} \frac{d t}{d T}-p
$$

which may be integrated in the form

$$
\frac{T}{T_{0}}=\int_{t_{0}}^{t} \frac{\left(\frac{\delta t}{\delta p}\right)_{v}}{\left(\frac{\delta U}{\delta v}\right)_{t}+p} \cdot d t
$$

where the temperatures $T$ and $T_{0}$ on the absolute scale correspond to $t$ and $t_{0}$ on the arbitrary scale. The quantities on the right hand side of this equation can be directly measured. The quantity $\left(\frac{\delta U}{\delta v}\right)_{t}$ is most conveniently determined from experiments on the Joule-Thomson effect.* A number of such determinations of $T_{0}$ the absolute zero have been carried out by a number of observers; in the Recueil de Constantes Physiques, published under the auspices of the Société Française de Physique, the most probable value is taken to be

$$
T_{0}=-273.09^{\circ} \mathrm{C} .
$$

From values of $p$ and $v$ for oxygen Berthelot (Trav. et Mém. Bur. Intl.) obtains

$$
R=8.315 \times 10^{7} .
$$

Equation (4) is useful in helping to interpret the equation for the velocity of translation of a molecule deduced from purely dynamical considerations in the next Section.

- For further information see Planck's Thermodynamics, pp. 124131, and Partington's Thermorlynymics, pp. 162-167. 


\section{The Velocity of Translation of a Molecule in} a Gas from Dynamics.

Consider a single molecule of absolute mass $m_{a}$ moving to and fro between two parallel walls at right angles to each with a velocity $V$. The molecule on colliding with one of the walls has its momentum changed by $2 \mathrm{Vm}_{a}$, and since this occurs $V / 2 l$ times per second, where $l$ denotes the distance between the walls, the change in momentum per second is equal to $\frac{V^{2} m_{a}}{l}$. Now according to dynamics

$$
\text { force } \times \text { time }=\text { change in momentum, }
$$

and the force exerted by the molecule on the wall is therefore equal to the foregoing expression. Suppose now that there are $N_{c}$ molecules per cubic $\mathrm{cm}$. moving in all directions between the walls. It will be convenient first to suppose that these molecules consist of three streams each equal to $N_{c} / 3$ moving parallel to three axes at right angles to each other. If one of these axes is taken at right angles to the walls, $l N_{c} / 3$ molecules exert the foregoing pressure on each square $\mathrm{cm}$. of the walls. Therefore if $p$ denotes this pressure we have

$$
p=\frac{N_{c} m_{a} V^{2}}{3}=\rho \frac{V^{2}}{3}, . . . . .
$$

where $N_{c} m_{a}=\rho$, the density of the gas.

Let us now suppose that the molecules move in all directions. Let us take a line at right angles to the walls and a point on the line, and draw a sphere of radius $r$ around it. Molecules will pass through the point in all directions, which is equivalent to supposing that $n_{a}$ molecules fall per square $\mathrm{cm}$. per second at right angles on the surface of the sphere. Let us find an expression for the fraction of the molecules passing through the point which move towards one of the 
walls and make an angle $\theta$ with it. On taking two lines making angles $\theta$ and $\theta+d \theta$ with the normal and rotating these lines with the normal as axis, a belt whose area is $2 \pi r \sin \theta \cdot r \cdot d \theta$ is traced out on the sphere. The fraction in question is therefore equal to

$$
\frac{2 \pi r \sin \theta \cdot r \cdot d \theta \cdot n_{a}}{2 \pi r^{2} n_{a}}=\sin \theta \cdot d \theta .
$$

Thus of the total number of molecules $N_{t}$ between the walls $N_{t} \sin \theta \cdot d \theta$ molecules make an angle $\theta$ with a normal to one of the walls and move towards it. Each of these molecules on striking the wall gives rise to a change in momentum equal to $2 m_{a} V \cos \theta$ and this happens $\frac{V \cos \theta}{2 l}$ times per second through rebounding from the two walls. The molecules under consideration therefore exert a pressure equal to

$$
\frac{N_{\imath} m_{a} V^{2} \cos ^{2} \theta \cdot \sin \theta \cdot d \theta}{l}
$$

upon the wall. The pressure $P$ exerted on the whole wall by the total number of molecules moving in all directions is therefore given by

$$
\begin{aligned}
P & =\frac{m_{a} N_{l} V^{2}}{l} \int_{0}^{\frac{\pi}{2}} \cos ^{2} \theta \sin \theta \cdot d \theta \\
& =-\frac{m_{a} N_{l} V^{2}}{l}\left[\frac{\cos ^{3} \theta}{3}\right]_{0}^{\frac{\pi}{2}}=\frac{m_{a} N_{t} V^{2}}{3 l} .
\end{aligned}
$$

If $A_{a}$ denotes the area of the wall, $p$ the pressure per square cm., and $N_{c}$ the number of molecules per cubic cm., we have

$$
P=A_{a} p, \text { and } N_{c}=\frac{N_{l}}{A_{a} l} \text {. }
$$

By means of these two equations the preceding equation may be reduced to equation (6). It appears therefore that 
we may represent the molecules, as far as the pressure they exert is concerned, by three streams moving at right angles to each other.

Since $p$ and $\rho$ can be directly measured, the velocity of translation of a molecule is at once given by

$$
V=\sqrt{\frac{3 p}{\rho}} . \cdots \cdot \cdot \cdot \cdot
$$

If $p$ is expressed in dynes and $\rho$ in grams per cc. the velocity $V$ is expressed in cms. per second.

In illustration of this formula the values of $V$ in $\mathrm{cms}$. per second for a few gases at standard temperature and pressure are given by Table I.

\section{TABLE I}

\begin{tabular}{|c|c|c|}
\hline Substance. & Formula. & $V \frac{\mathrm{cms} .}{\mathrm{sec} .}$ \\
\hline 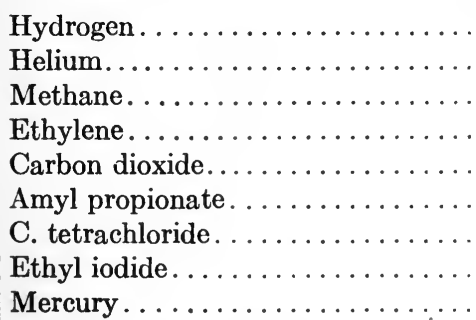 & $\begin{array}{c}\mathrm{H}_{2} \\
\mathrm{He} \\
\mathrm{CH}_{4} \\
\mathrm{C}_{2} \mathrm{H}_{4} \\
\mathrm{CO}_{2} \\
\mathrm{C}_{8} \mathrm{H}_{16} \mathrm{O}_{2} \\
\mathrm{CCl}_{4} \\
\mathrm{C}_{2} \mathrm{H}_{5} \mathrm{I} \\
\mathrm{Hg}\end{array}$ & $\begin{array}{r}169,200 \\
120,400 \\
60,060 \\
45,420 \\
36,250 \\
20,030 \\
19,390 \\
18,820 \\
17,000\end{array}$ \\
\hline
\end{tabular}

Since in the foregoing investigation the velocity of a molecule does not depend on the presence of another molecule, $V$ is independent of the volume of the gas. 'Therefore according to equation (6) the pressure of a gas is proportional to its density, or inversely proportional to its volume, which agrees with the facts and is known as Boyle's law. 
By means of equation (4) the foregoing equation may be written

$$
V=\sqrt{\frac{3 R T}{m}} \cdot \cdot \cdot \cdot \cdot \cdot \cdot
$$

The velocity of translation of a molecule thus varies inversely as the square root of its mass, and hence the greater the mass the smaller the velocity. It is due to this that it is possible to observe directly the motion of large particles in a liquid, as described in Section 4. The velocity is also proportional to the square root of the absolute temperature, and hence is equal to zero at the absolute zero of temperature.

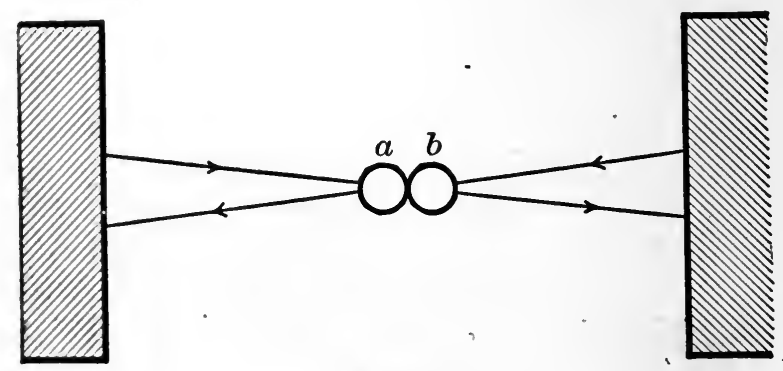

Fig. 1.

In the foregoing investigation it has been tactily assumed that the apparent velocity of translation of a molecule is not effected by the presence of other molecules. This, however, does not hold; the velocity is apparently increased by the volumes of the molecules. Thus consider two molecules, $a$ and $b$, which collide head on so that each retraces its path as shown in Fig. 1. This amounts to the same thing as if the molecules at the moment of collision were to be interchanged, the molecule $a$ then taking the place of the retreating molecule $b$, and the molecule $b$ the place of the retreating molecule $a$. The velocity of each molecule is thus apparently increased, since each passes instantaneously over 
a part of its path equal to the diameter of a molecule. Under these conditions the walls are struck oftener per second by each molecule, giving rise to a greater pressure than would be obtained if each molecule had no volume or space associated with it through which another molecule cannot pass. The values of $V$ given by equation (7) are therefore greater than they should be. It is easy to see, however, that if the diameter of a molecule is small in comparison with the distance of separation of the molecules, as is the case with a gas under ordinary conditions, the error introduced in this way is small. It will be evident now that the deviation of a gas from equation (7) is in part caused by the apparent volume associated with each molecule. The remaining part of the deviation is caused by the molecular forces, which will be discussed in Sections 14, 15, and 21.

It has also been assumed that each molecule possesses the same velocity. This does not hold in practice in a gas, since the interaction between the molecules tends to continually change their velocities. A theoretical investigation showing that this is the case, and the law of distribution of velocities obtained by Maxwell, will be given in the next Section. But we may still express $p$ by means of equation (6), where $V$ is now called the average kinetic energy velocity, a quantity which will be discussed in Section 8 . It is the velocity which gives the average kinetic energy of a molecule.

\section{Maxwell's Law of Distribution of the Veloci-} ties of the Molecules of a Gas.

It can be shown theoretically that the molecules in a gas at any instant have not the same velocity; moreover, the velocity of each molecule is continually changing in magnitude. This is what we would expect on account of the interaction between the molecules. According to Maxwell's law, which will be proved presently, the number of 
molecules $N_{1}$ of $N$ molecules which have a velocity lying between $V_{1}$ and $V_{1}+d V_{1}$ at any instant is given by

$$
N_{1}=\frac{4 N V_{1}^{2}}{\sqrt{\pi} V_{p}^{3}} e^{-\left(\frac{V_{1}}{V_{p}}\right)^{2}} \cdot d V_{1} . \quad . \quad . \quad .
$$

The most probable velocity is the value of $V_{1}$ which gives the foregoing expression a maximum value. Therefore if the differential coefficient of the expression with respect to $V_{1}$ is obtained and equated to zero we have

$$
V_{1}=V_{p} \text {. . . . . . . }
$$

Thus $V_{p}$ is the most probable velocity that occurs amongst the gas molecules.

The probability of the occurrence of other velocities decreases rapidly on increasing or decreasing the value of $V_{1}$ from $V_{p}$. That a molecule may have an infinitely small velocity is of zero probability, and this also holds for an infinitely large velocity. It appears that by far the larger number of molecules have velocities differing but little from the most probable velocity, and as a first approximation we may therefore suppose that the molecules have the same velocity.

The most probable velocity $V_{p}$ must not be confounded with the average velocity $V_{a}$. The latter quantity is obtained by adding up all possible velocities and dividing their number into the sum obtained. This corresponds to multiplying the expression for $N_{1}$ from equation (9) by $V_{1}$, integrating it between the limits $V_{1}=0$ and $V_{1}=\infty$, and dividing by $N$, giving

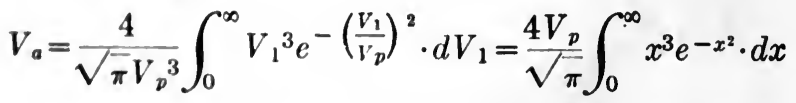

$$
\begin{aligned}
& =\frac{4 V_{\nu}}{\sqrt{ } \frac{1}{\pi}}\left[-\frac{x^{2} e^{-x^{2}}}{2}+\int x e^{-x^{2}} \cdot d x\right]_{0}^{\infty}=\frac{2 V_{p}}{\sqrt{\pi}}, .
\end{aligned}
$$


on writing $V_{1} / V_{p}=x$. Thus the average velocity is greater than the most probable.

Maxwell's law can at once be strictly deduced if it can be shown that the velocity components of a molecule along three axes at right angles are indspendent of each other. It will be recognized that this need not necessarily hold without being proved, on account of the interaction between the molecules. Maxwell's law has therefore been deduced by a number of mathematicians in different ways from a general investigation of molecular motion, but which all involve one or more assumptions which are stated, or partly hidden in the deductions. Mathematicians are therefore not yet agreed that a perfect proof has been given, while some maintain that the law cannot hold. The writer has developed a thermodynamical proof that the velocity components mentioned should be independent, and on this the proof of Maxwell's law will be based in this book.

It can be shown from thermodynamics that osmotic equilibrium is not of a temporary nature, but must be permanent. A number of thermodynamical formulæ have been deduced involving the isothermal separation of substances by means of semipermeable membranes. Van't Hoff's formula for the heat of formation of a substance in the gaseous state, for example, depends upon the properties of semipermeable membranes. The thermodynamical equation (47) in Section 21 may evidently be applied to a system involving semipermeable membranes. Now the isothermal separation of substances must take place infinitely slowly only, otherwise the process would not be isothermal. Therefore the gases on the two sides of the semipermeable membrane of a system must be in permanent equilibrium with each other when the movable parts of the system are kept in a fixed position, that is, in the limiting case when the movable parts are kept in fixed positions the diffusion of the different gaseous constituents from one side of the 
membrane to the other must be infinitely small. If this were not the case the formulæ deduced would not apply in practice. Thus in the limiting case the diffusion of certain gases through a given membrane may be infinitely small, while other gases pass readily. Each of the constituents of a gaseous mixture may thus exist on the two sides of a membrane, when initially placed on one side, but the condition of the system may be such that some of them exist on the other side of the membrane in infinitely small amounts only, which condition remains unaltered.

The partial pressure of each constituent of a mixture of gases is the same as if the other gases were absent. Hence the redistribution of the velocities of the molecules of a gas that might take place on addition of another gas is such that each molecule produces the same average pressure as before, which is equivalent to each molecule having the constant velocity $V$ given by equation (7), which will be called the pressure velocity in this Section.

Let us now consider a mixture of molecules $a$ and $b$ in a chamber $A B$ separated from a chamber $A$ by a semipermeable membrane, which is permeable only to the molecules $a$. A migration of the molecules $a$ from the chamber $A$ through the membrane into the chamber $A B$, and in the reverse direction is continually going on. This is shown by the fact that on increasing the volume of the chamber $A$ molecules $a$ at once pass into it from the chamber $A B$, and that therefore there is a free passage for the molecules $a$ through the membrane which can only be interfered with occasionally by molecules coming in the opposite direction. The number of molecules passing in one direction per second is evidently equal to the number of molecules passing in the opposite direction, otherwise the number of molecules on each side of the membrane would gradually change. We have seen that the pressure velocity $V$ of translation of the molecules $a$ is the same in the two chambers. The 
pressure velocity of each set of migrating molecules must also be the same and equal to the foregoing velocity, otherwise the pressure would gradually change in the two chambers. Let $N^{\prime}$ denote the number of molecules migrating from one chamber into the other when no molecules $b$ are in the chamber $A B$, and $N^{\prime \prime}$ the number corresponding to a given number of molecules $b$ in the chamber. Thus the addition of the molecules $b$ to the chamber $A B$ has the effect of dividing the number of molecules $N^{\prime}$ into two parts, the part $N^{\prime \prime}$ which migrates and the part $N^{\prime}-N^{\prime \prime}$ which does not, the pressure molecular velocity being the same for each part according to what has gone before. Now a set of molecules of different velocities can be divided in one way only into two parts satisfying the foregoing conditions, viz.: which corresponds to the same distribution of velocities in each part. For if the number of molecules $N^{\prime \prime}$ is decreased by $n^{\prime}$, the pressure velocity of these $n^{\prime}$ molecules must be equal to $V$, since this holds for the molecules $N^{\prime \prime}-n^{\prime}$ and $N^{\prime}-$ $\left(N^{\prime \prime}-n^{\prime}\right)$, which can only be realized in the foregoing way unless each molecule $a$ has the same velocity. Thus the distribution of velocities between the molecules migrating from the chamber $A$ into the chamber $A B$ is the same as that of the molecules in the chamber $A$, and the distribution amongst the molecules migrating from the chamber $A B$ into the chamber $A$ is the same as that of the molecules in the chamber $A B$. These two distributions can be shown to be identical. If this were not the case the molecules passing into the chamber $A$ would give rise to a different distribution of velocities near the membrane than exists at other parts of the chamber. This would effect the migration of the molecules $a$ from the chamber $A$ into the chamber $A B$, and thus disturb the equilibrium. From this it follows that the distribution of velocities between the molecules $a$ in the chamber $A$ is the same as that in the chamber $A B$. The addition of molecules $b$ to the chamber $A B$, accord- 
ing to the foregoing investigation, does not alter the distribution of the velocities between the molecules $a$ from that obtained when molecules $a$ only are in the chamber. Thus the velocity components at right angles to each other of the molecules $a$ are not changed by collision with the molecules $b$, and hence not changed by the collision of the molecules $a$ with each other, i.e., the velocity components are independent of each other. This result will now be used to prove Maxwell's law.

Let the rectangular components of the velocity $V_{1}$ of a molecule be denoted by $a, b$, and $c$, in which case

$$
V_{1}^{2}=a^{2}+b^{2}+c^{2} \text {. . . . . . }
$$

Let the probability that the component velocity along the $x$ axis has a value lying between $a$ and $a+d a$ be expressed by the function $f(a)$, similarly let $f(b)$ and $f(c)$ express the probabilities that the component velocities along the $y$ and $z$ axes lie between $b$ and $b+d b$, and between $c$ and $c+$ $d c$, respectively. The probability of the three components occurring simultaneously is therefore $f(a) \cdot f(b) \cdot f(c)$, since the components are independent. The situation of the system of coordinates in space is arbitrary, and therefore

$$
f(a) \cdot f(b) \cdot f(c)=\phi_{1}\left(a^{2}+b^{2}+c^{2}\right),
$$

where $\phi_{1}$ is a definite function of $V_{1}{ }^{2}$. On differentiating this equation, keeping $V_{1}$ constant, and dividing the resultant equation by $f(a) \cdot f(b) \cdot f(c)$, we obtain

$$
\frac{f^{\prime}(a)}{f(a)} \cdot d a+\frac{f^{\prime}(b)}{f(b)} \cdot d b+\frac{f^{\prime}(c)}{f(c)} \cdot d c=0 .
$$

The differentiation of equation (12) under the same condition gives

$$
a \cdot d a+b \cdot d b+c \cdot d c=0 .
$$


On multiplying this equation by the undetermined multiplier $\lambda$, the foregoing two equations may be combined into the equation

$$
\left(\frac{f^{\prime}(a)}{f(a)}+\lambda a\right) d a+\left(\frac{f^{\prime}(b)}{f(b)}+\lambda b\right) d b+\left(\frac{f^{\prime}(c)}{f(c)}+\lambda c\right) d c=0 .
$$

Since the changes $d a, d b$, and $d c$, are independent their factors may be separately equated to zero, giving

$$
\frac{f^{\prime}(a)}{f(a)}+\lambda a=0,
$$

and two similar equations involving $b$ and $c$. The integration of these equations gives $f(a)=C_{1} e^{-\frac{\lambda}{2} a^{2}}, f(b)=C_{1} e^{-\frac{\lambda}{2} b^{2}}$, and $f(c)=C_{1} e^{-\frac{\lambda}{2} c^{2}}$, where $C_{1}$ denotes an arbitrary constant whose value is infinitely small, since the probability of a certain case in an infinitely large number of possible cases is infinitely small. We may therefore write $C_{1}=C_{2} \cdot d a$, and if besides we write $\frac{\lambda}{2}=\left(\frac{1}{V_{p}}\right)^{2}$ we have

$$
f(a)=C_{2} e^{-\left(\frac{a}{V_{p}}\right)^{2}} \cdot d a .
$$

Since the sum of the probability of an event happening and the probability of it failing is equal to unity, we have for all possible cases

$$
C_{2} \int_{-\infty}^{\infty} e^{-\left(\frac{a}{V_{p}}\right)^{2}} \cdot d a=1
$$

the integral expressing the sum of all the probabilities. Similarly we have

$$
C_{2} \int_{-\infty}^{\infty} e^{-\left(\frac{b}{V_{p}}\right)^{2}} \cdot d b=1
$$


which on multiplying by the preceding equation becomes

$$
C_{2}{ }^{2} \int_{-\infty}^{\infty} \int_{-\infty}^{\infty} e^{-\frac{a^{2}+b^{2}}{V p^{2}}} \cdot d a \cdot d b=1
$$

or

$$
C_{2}^{2} V_{p}^{2} \int_{-\infty}^{\infty} \int_{-\infty}^{\infty} e^{-\left(x^{2}+y^{2}\right)} d x \cdot d y=1
$$

if we write $a / V_{p}=x$ and $b / V_{p}=y$. If $x$ and $y$ are now regarded as coordinates in a system of rectangular coordinates they may be transformed into polar coordinates by writing

$$
x^{2}+y^{2}=r^{2} \text { and } d x \cdot d y=r \cdot d r \cdot d \phi,
$$

which transforms the foregoing integral into

and hence

$$
C_{2}^{2} V_{p}^{2} \int_{0}^{\infty} \int_{0}^{\pi} e^{-r^{2}} r \cdot d r \cdot d \phi=\pi C_{2}^{2} V_{p}^{2}=1,
$$

Accordingly

$$
C_{2}=\frac{1}{V_{p} \sqrt{\pi}}
$$

$$
\begin{gathered}
f(a)=\frac{1}{V_{D} \sqrt{\pi}} e^{-\left(\frac{a}{V_{p}}\right)^{2}} \cdot d a, \quad f(b)=\frac{1}{V_{D} \sqrt{\pi}} e^{-\left(\frac{b}{V_{p}}\right)^{2}} \cdot d b, \\
f(c)=\frac{1}{V_{p} \sqrt{\pi}} e^{-\left(\frac{c}{V_{D}}\right)^{2}} \cdot d c,
\end{gathered}
$$

and the probability that the three components $a, b$, and $c$, occur simultaneously is therefore

$$
f(a) \cdot f(b) \cdot f(c)=\frac{1}{V_{p}^{3} \pi^{3 / 2}} e^{-\frac{a^{2}+b^{2}+c^{2}}{V^{2} p}} \cdot d a \cdot d b \cdot d c .
$$

To obtain the probability of the occurrence of a certain velocity $V_{1}$ let us as before take $a, b$, and $c$, as coordinates, in which case

$$
V_{1}^{2}=a^{2}+b^{2}+c^{2}
$$


and

$$
d a \cdot d b \cdot d c=V_{1}^{2} \cdot d V_{1} \cdot \sin \theta \cdot d \theta \cdot d \phi,
$$

where $\theta$ denotes the angle between $V_{1}$ and the $c$ axis, and $\phi$ the angle between the $a$ axis and the projection of $V_{1}$ on the $(a, b)$ plane. The probability of a velocity $V_{1}$ having a definite direction is therefore

$$
\frac{1}{V_{p}^{3} \pi^{3 / 2}} e^{-\left(\frac{V_{1}}{V_{p}}\right)^{2} V_{1}^{2} \cdot d V_{1} \cdot \sin \theta \cdot d \theta \cdot d \phi .}
$$

The probability independent of any direction is obtained on integrating with respect to $\theta$ from 0 to $\pi$, and with respect to $\phi$ from 0 to $2 \pi$, which is taking into account all possible directions. This can easily be shown to give

$$
\frac{4}{V_{p_{s}}^{3} \sqrt{ } \pi} V_{1}^{2} e^{-\left(\frac{V_{1}}{V_{p}}\right)^{2}} \cdot d, V_{1}
$$

which expresses the probability that a molecule has a velocity lying between $V_{1}$ and $V_{1}+d V_{1}$. The fraction of $N$ molecules possessing this velocity is then immediately given by equation (9), which completes the proof.

It will easily be seen that the average velocity of translation of a molecule depends on the nature of the distribution of the velocities amongst the molecules. But the average kinetic energy of a molecule is independent of this distribution, as will appear from the next Section.

8. The Average Kinetic Energy Velocity of a Molecule, and the Relation between the Kinetic Energy of a Molecule and its Absolute Temperature.

If $N_{1}$ molecules in a cubic $\mathrm{cm}$. of a gas have a velocity. $V_{1}$, and $N_{2}$ molecules a velocity $V_{2}$, etc., where

$$
N_{1}+N_{2}+\ldots N_{n}=N_{c} \text {, }
$$


the total number of molecules in the cubic $\mathrm{cm}$., the average kinetic energy of a molecule is given by

$$
\frac{1}{2} m_{a}\left(\frac{N_{1} V_{1}^{2}+N_{2} V_{2}^{2}+\ldots N_{n} V_{n}^{2}}{N_{1}+N_{2}+\ldots N_{n}}\right),
$$

where $m_{a}$ denotes the absolute mass of a molecule. This expression for the kinetic energy may also be written

$$
\frac{1}{2} m_{a} V^{2},
$$

where $V$ will be called the average kinetic energy velocity of a molecule, and is given by

$$
V=\sqrt{\frac{N_{1} V_{1}^{2}+N_{2} V_{2}^{2}+\ldots N_{n} V_{n}^{2}}{N_{1}+N_{2}+\ldots N_{n}}} .
$$

This velocity is evidently not equal to the average velocity $V_{a}$, which is given by

$$
V_{a}=\frac{N_{1} V_{1}+N_{2} V_{2}+\ldots N_{n} V_{n}}{N_{1}+N_{2}+\ldots N_{n}} .
$$

It can be shown that the velocity $V$ is independent of the distribution of the molecular velocities. Thus if $p_{1}$ denote the partial pressure of the $N_{1}$ molecules having a velocity $V_{1}$, and $p_{2}$ the partial pressure of the $N_{2}$ molecules having a velocity $V_{2}$, etc., the total pressure $p$ of the gas is given by

$$
p=p_{1}+p_{2} \ldots p_{n} .
$$

But according to equation (6)

$$
\begin{aligned}
& p_{1}=\frac{N_{1} m_{a}}{3} V_{1}{ }^{2}, \\
& p_{2}=\frac{N_{2} m_{a}}{3} V_{2}{ }^{2},
\end{aligned}
$$


and

$$
p_{n}=\frac{N_{n} m_{a}}{3} V_{n}^{2}
$$

and hence

$$
p=\frac{m_{a}}{3}\left(N_{1} V_{1}^{2}+N_{2} V_{2}^{2}+\ldots N_{n} V_{n}^{2}\right)=\frac{N_{c} m_{a}}{3} V^{2} .
$$

This equation, which is same as equation (6), expresses $V$ in terms of $p$ and $m_{a}$, and it is therefore independent of the distribution of molecular velocities.

The kinetic energy of a gram molecule of molecules in the gaseous state therefore becomes

$$
\frac{1}{2} m V^{2}=\frac{3 R}{2} T=\gamma T=1.247 \times 10^{8} T \mathrm{ergs}, \quad \cdot
$$

by the help of equation (8). The average kinetic energy of a single molecule, which is $1 / N$, or $1 / 6.2 \times 10^{23}$, the foregoing value, is therefore equal to

$$
\frac{1}{2} m_{a} V^{2}=\frac{3 R}{2 N} T=2.012 \times 10^{-16} T \text { ergs. . . }
$$

The values of $R$ and $N$ used are given in Sections 5 and 3 . Thus the average kinetic energy of a molecule, whatever the distribution of molecular velocities, is simply proportional to the absolute temperature, and thus independent of molecular mass.

It will be of interest to obtain the connection between the average kinetic energy velocity $V$ of a molecule and its most probable velocity $V_{p}$ according to Maxwell's law. The number of molecules of $N_{c}$ molecules in a cubic $\mathrm{cm}$. whose velocities lie between $V_{1}$ and $V_{1}+d V_{1}$ at any instant is equal to

$$
\frac{4 N_{c} V_{1}^{2}}{\sqrt{\pi} V_{p}^{3}} e^{-\left(\frac{V_{1}}{V_{p}}\right)^{2}} \cdot d V
$$


according to the preceding Section. The pressure these molecules exert is obtained by multiplying this expression by $\frac{m_{a}}{3} V_{1}^{2}$, according to equation (6). Therefore on integrating the foregoing product from $V_{1}=0$ to $V_{1}=\infty$ we obtain the total pressure $p$ exerted by the molecules, that is

$$
\begin{aligned}
& p=\frac{4 m_{a} N_{c}}{3 \sqrt{\pi} V_{p}^{3}} \int_{0}^{\infty} V_{1}^{4} e^{-\left(\frac{V_{1}}{V_{p}}\right)^{2}} \cdot d V_{1}=\frac{4 m_{a} N_{c} V_{p}^{2}}{3 \sqrt{\pi}} \int_{0}^{\infty} x^{4} e^{-x^{2}} \cdot d x \\
& =\frac{6 m_{a} N_{c} V_{p}^{2}}{3 \sqrt{\pi}} \int_{0}^{\infty} x^{2} e^{-x^{2}} \cdot d x=\frac{3 m_{a} N_{c} V_{p}^{2}}{3 \sqrt{\pi}} \int_{0}^{\infty} e^{-x^{2}} \cdot d x \\
& =\frac{N_{c} m_{a} V_{p}^{2}}{2}=\frac{\rho}{2} V_{p}^{2},
\end{aligned}
$$

since $\int_{0}^{\infty} e^{-x^{2}} \cdot d x=\frac{\sqrt{\pi}}{2}$, where $\rho$ denotes the density. On comparing the foregoing value of $p$ with that given by equation (6) we obtain

$$
V=\left(\frac{3}{2}\right)^{1 / 2} V_{p}=1.23 V_{p}
$$

or the average kinetic energy velocity is about $23 \%$ greater than the most probable velocity. The relation between $V$ and $V_{a}$ is obtained from a comparison of the foregoing equation and equation (11), which gives

$$
V_{a}=\sqrt{\frac{8}{3 \pi}} V=.922 \mathrm{~V} .
$$

Thus the average velocity is about $92 \%$ of the average kinetic energy velocity.

In the case of a mixture of gases of molecules $r$ and $e$ the total pressure $p$ is given by

$$
p=p_{e}+p_{r}=\frac{N_{\epsilon} m_{a e} V_{e}^{2}}{3}+\frac{N_{r} m_{a r} V_{r}^{2}}{3},
$$


on applying equation $(6)^{*}$, where $p_{e}$ and $p_{r}$ denote the partial pressures, $N_{e}$ and $N_{r}$ the partial concentrations, $m_{a e}$ and $m_{a r}$ the absolute molecular weights, and $V_{e}$ and $V_{r}$ the average kinetic energy velocities of the molecules $e$ and $r$ respectively. If the temperature of each set of molecules is the same an application of equation (8) to the foregoing equation gives

$$
p=N_{e} \frac{m_{a e}}{m_{e}} R T+N_{r} \frac{m_{a r}}{m_{r_{r}}} R T=N_{e r} \frac{R T}{N},
$$

where $\mathrm{N}_{e r}$ denotes the total concentration of the molecules per cubic cn., and $m_{e} / m_{a e}=m_{r} / m_{a r}=N$ the number of molecules in a gram molecule of a pure substance. This equation, which is the same as equation (5), agrees with the facts, showing that when two gases are mixed they rapidly assume the same temperature. This would arise in part through both gases being in contact with the vessel whose temperature they would gradually assume, in other words, the vessel usually being a conductor of heat would act as an intermediary in adjusting the gases to the same temperature. The question then arises, would an equalization of temperature take place through the interaction of the molecules alone, and what is the distribution of velocities when the two sets of molecules in some way or other have acquired the same temperature? This will be discussed in the next Section.

9. The Equipartition of Energy between the Different Molecules in a Mixture of Gases.

When two sets of molecules of different kinds at different temperatures are mixed, the average kinetic energy of each molecule of each set, according to the Law of Equipartition of Energy, eventually becomes the same through molec-

${ }^{*}$ We may do this since each set of molecules acts independently. 
ular interaction, and the corresponding distribution of the molecular velocities at any instant is the same as if the gases were isolated. This law is usually proved, or attempts are made to prove it, from purely dynamical considerations in treatises on the Kinetic Theory of Gases. The analysis is very intricate, and the suppositions introduced are not unobjectionable. The subject has always attracted a good deal of attention from mathematicians with the object of putting the proof on a sounder basis. In the earlier development of the subject the investigations of Maxwell and Baltzmann are pre-eminent.

The dynamical proof of the law usually depends on the assumption that the molecules consist of perfectly elastic spheres not surrounded by fields of force, and that the equipartition of energy is solely caused by molecular collision. As a matter of fact in practice the equipartition would be caused in other ways if not caused by molecular collision. Thus we know from experience that a mass of a hot gas located in a colder gas radiates heat to the latter till the temperature is equalized. A well-known example of this is the radiation manifested by the hot air rising from a fire, or from a furnace. This shows that each individual molecule in a gas is continually radiating heat energy whose amount per second depends on its kinetic energy. Therefore if two sets of molecules at different temperatures are mixed heat will be radiated from one set of molecules to the other till the average kinetic energy of each molecule is the same, or the two sets of molecules have the same temperature, as would be the case if the two sets of molecules were separate but adjacent to each other. It will follow then from Section 7 that after temperature equilibrium has been obtained the distribution of velocities in each set of molecules is the same as if it were isolated.

It seems unnecessary and futile, therefore, to endeavor to establish the Iaw of Equipartition of Energy on assumptions 
relating to the interaction of molecules, when the law follows directly from the fact that a molecule is continually radiating heat energy. Although equipartition of energy would be brought about as indicated, it is very likely that it would also be brought about by the collision of elastic spheres, since it follows from mechanics that on the average two colliding bodies of different kinetic energies have their energies more evenly distributed after collision. In fact, every kind of interaction between two molecules, whether by "collision," or through the molecular forces of attraction and repulsion, has on the average the effect of redistributing the kinetic energy in favor of the body possessing the lesser energy. A definite mathematical proof that equipartition of energy may take place along these lines is difficult on account of having to deal with a large number of molecules possessing different velocities, each of which interacts some time with one or more molecules, and it is therefore necessary to show that equilibrium in energy partition exists after an infinite time beginning from an indefinite state of affairs.

\section{The Number of Molecules per Cubic Cm. in a Gas.}

According to equation (5) the number $N_{c}$ of molecules per cubic $\mathrm{cm}$. of a gas is given by

$$
N_{c}=\frac{\rho}{m_{a}}=\frac{N}{R} \frac{p}{T}=7.46 \times 10^{15} \frac{p}{T}, \quad . \quad .
$$

where $N$ and $R$ are given in Sections 3 and 5 . Hence if two different gases possess the same temperature and pressure each contains the same number of molecules per cubic cm. This result is known as Avogadro's Law. It follows also that two gases at different temperatures contain the same number of molecules per cubic $\mathrm{cm}$. if the ratio $p / T$ has the same value for both. It will appear 
from Section 5 that these results also hold for mixtures of gases, in which case $p$ denotes the sum of the partial pressures.

The foregoing equation may also be written

$$
N_{c}=N \frac{\rho}{m}=6.2 \times 10^{23} \frac{\rho}{m}, \quad \cdot \quad \cdot \quad \cdot
$$

according to equation (5), from which it follows that two gases not necessarily at the same temperature contain the same number of molecules per cubic $\mathrm{cm}$. if they have the same values for the ratio $\rho / m$.

Since the molecules possess motion of translation this leads us to the consideration of another quantity.

\section{The Number of Molecules Crossing a Square} $\mathrm{Cm}$. in all Directions from one Side to the Other in a Gas.

This number is of importance, since it is one of the fundamental quantities occurring in the different formulæ relating to a gas. It can be expressed in terms of quantities which can be measured directly, and hence its numerical value obtained when desired. We will see in Section 29 that its value can also be found in the case of a liquid. It will be convenient first to obtain the number corresponding to the supposition that the molecules move parallel to three axes at right angles to each other. We have seen that this supposition may be made when dealing with the connection between the velocity of translation of the molecules and the pressure which they exert. Let $n_{0}$ denote the number of molecules crossing a plane one square $\mathrm{cm}$. in area per second in one direction situated at right angles to one of the foregoing axes. It follows then that

$$
n_{0}=\frac{N_{c} V_{a}}{2 \times 3}=\frac{\rho V_{a}}{6 m_{a}}, \quad \text {. . . . . }
$$


where $V_{a}$ denotes the average velocity of a molecule, $N_{c}$ the molecular concentration, and $\rho$ the density of the gas. To prove this we may suppose that we are dealing with a single cubic $\mathrm{cm}$. of gas whose faces are parallel to the axes mentioned, in which case a molecule having a velocity $V_{x}$ will cross it $V_{x} / 2$ times in one direction per second. Therefore if there are $N_{1}, N_{2}, N_{3} \ldots$, molecules having respectively the velocities $V_{1}, V_{2}, V_{3} \ldots$, we have

$$
\begin{aligned}
n_{0} & =\frac{1}{3 \times 2}\left\{N_{1} V_{1}+N_{2} V_{2}+\ldots\right\} \\
& =\frac{N_{c}}{6}\left\{\frac{N_{1} V_{1}+N_{2} V_{2}+\ldots}{N_{c}}\right\}=\frac{N_{c} V_{a}}{6} .
\end{aligned}
$$

Another expression for $n_{0}$ which is very useful may be obtained. The pressure $p$ in dynes exerted by a gas may be written

$$
p=n_{0} A, \quad . \quad . \quad . \quad . \quad .
$$

where $A$ represents an appropriate factor, which has other important applications which will be found in Section 20 . The value of the quantity $A$ may be determined by substituting in the foregoing equation successively for the quantities $p, n_{0}$ and $V_{a}$, assuming that $V_{a}=V$, from the equations (17), (8), and (4), giving

$$
A=6 \frac{m_{a}}{m} \sqrt{\frac{R}{3}} \sqrt{T m}=5.087 \times 10^{-20} \sqrt{T m}, \quad .
$$

where $m_{a} / m=1.61 \times 10^{-24}$, the absolute value of the mass of a hydrogen atom.

Let us now consider the case when the molecules are moving in all directions. Let a point $b$ be taken on a plane $a b c$ in the gas, Fig. 2, and a sphere of radius $r$ described round it. The molecules passing through the point $b$ strike the surface of the sphere at right angles, and let us therefore 
suppose that their number corresponds to $S$ molecules entering the sphere per second per $\mathrm{cm} .^{2}$ of its surface. The number of molecules impinging on a circular belt of the sphere of breadth $r \cdot d \theta$, and radius $r \cos . \theta$ is therefore $2 \pi r^{2} S$ $\cos \theta \cdot d \theta$. The force exerted by a molecule impinging on the plane $a b c$ at an angle $\theta$ is proportional to its component velocity at right angles to the plane according to equations (17) and (18), and thus according to equation (18) equal to $\frac{V \sin \theta}{V} \cdot A$, or $\sin \theta \cdot A$. Hence the molecules entering the

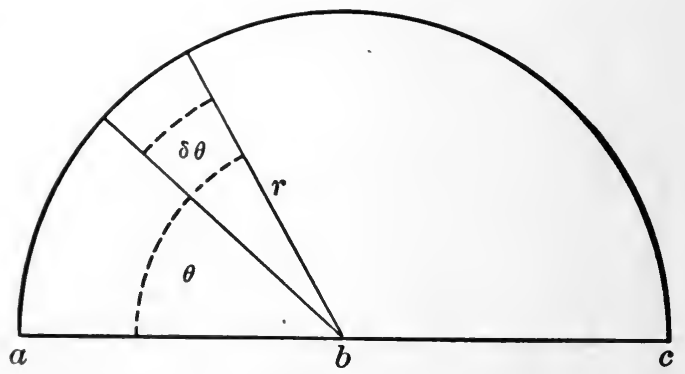

FIG. 2.

upper hemisphere, which lie in the solid angle $\pi$, on impinging on the point $b$ exert the force

$$
\int_{0}^{\frac{\pi}{2}} 2 \pi r^{2} S A \sin \theta \cdot \cos \theta \cdot d \theta=\pi r^{2} S A .
$$

If $u$ denotes this number of molecules we have $u=2 \pi r^{2} S$, and the pressure exerted may therefore be written $u \cdot A / 2$. Hence if $n$ denotes the number of molecules crossing a square $\mathrm{cm}$. from one side to the other in all directions, the pressure $p$ of the gas is given by

$$
p=n \frac{A}{2}=n 2.543 \times 10^{-20} \sqrt{T m} \quad . \quad . \quad .
$$


This equation may be used to find $n$. The equation may also be written in the form

$$
n=\frac{1}{v} \frac{1}{m_{a}} \sqrt{\frac{R T m}{3}}, \quad . \quad . \quad .
$$

by means of equations (19) and (4), where $v$ denotes the volume of a gram molecule of molecules.

In the foregoing investigation we have assumed that $V_{a}=V$, or the average velocity of translation of a molecule is equal to the average kinetic energy velocity. But this is not the case according to Section 8. According to Maxwell's law $V_{a}=\sqrt{\frac{8}{3 \pi}} V=.922 \mathrm{~V}$. If this is accepted and taken into account the value of $A$ is $5.521 \times 10^{-20} \sqrt{T m}$, or 1.085 times that given by equation (19). In subsequent investigations we shall, however, use the value of $A$ given by equation (19). The necessary change if desired can always be readily made.

The value of $n$ given by equation (21) is corrected according to Maxwell's law by multiplying the right-hand side of the equation by $\sqrt{\frac{8}{3 \pi}}$, or by .922 .

Since a molecule in motion represents a certain amount of kinetic energy, general energy considerations will be of interest and importance.

\section{The First Law of Thermodynamics.}

According to this law energy is indestructible. Since the expenditure of energy on a substance is accompanied by a change in temperature heat represents a form of energy. The amount of change in temperature depends on the heat capacity, or the specific heat, of the substance. By definition the heat capacity of water between the temperatures 
$14.5^{\circ} \mathrm{C}$. and $15.5^{\circ} \mathrm{C}$. is unity, and the amount of heat absorbed corresponding to this change in temperature is called a calorie. Other definitions of the calorie have been proposed, but the foregoing is mostly used. The units of heat capacity and mechanical work are quite arbitrary, and therefore a factor according to the above law should exist which would enable heat units to be converted into mechanical units, and vice versa, that is, if $W$ denotes the amount of work in ergs expended to produce an amount of heat $Q$ in calories, the relation between the two quantities is expressed by the equation

$$
W=J Q,
$$

where $J$ denotes the factor in question.

The first law of thermodynamics was first enunciated by Mayer in 1842, who obtained a value for $J$ from the difference between the specific heats of air at constant pressure and at constant volume, which is equal to $R / J$ according to the next Section. The value obtained is not very accurate on account of the existence of the Joule-Thomson effect according to which a certain amount of work is done in overcoming molecular attraction during the expansion of a gas. But it would have been possible to obtain a much better value by this method by using the gas at a temperature and pressure for which the Joule-Thomson effect is zero; the method has, however, now been superseded by others. In 1843 Joule began a series of classical experiments to test the law from different aspects, the best values of $J$ being obtained from the experiments on the agitation of liquids. More recently $J$ has been determined from the heating produced by an electric current. In the Recuiel de Constants Physiques (Gauthier-Villars, 1913) published under the ảuspices of the Société Française de Physique, its most probable value is taken to be

$$
J=4.184 \times 10^{7} .
$$


We may now consider more closely the nature of heat capacity of substances.

\section{The Specific Heats of Gases and Liquids.}

The specific heat of a perfect gas at constant volume consists of the sum of the changes in kinetic energy of motion of translation and internal molecular energy per degree change in temperature. If the specific heat is expressed in calories, the first term is equal to $3 R / 2 J$ for a gram molecule according to the preceding Section, and equation (13); the second term may be written $\left(\frac{\delta u_{a}}{\delta T}\right)_{0}$, where $u_{a}$ denotes the internal molecular energy per gram molecule expressed in terms of calories. Hence if $S^{\prime}{ }_{0}$ and $S_{v}$ denote the specific heats per gram molecule and per gram respectively we have

$$
S^{\prime}{ }_{0}=\frac{3 R}{2 J}+\left(\frac{\delta u_{a}}{\delta T}\right)_{0}, \ldots . . .
$$

and

$$
S_{\imath} m=S_{{ }^{\prime}}^{\prime} \quad \text {. . . . . . }
$$

If the pressure of the gas is kept constant during the change of temperature additional heat is absorbed in doing the external work $\frac{p}{J}\left(\frac{\delta v}{\delta T}\right)_{p}$ expressed in calories, which is equal to $R / J$ according to equation (4). Therefore if $S^{\prime}{ }_{p}$ and $S_{p}$ denote the specific heats at constant pressure per gram molecule and per gram respectively we have

$$
S_{p}^{\prime}=\frac{5 R}{2 J}+\left(\frac{\delta u_{a}}{\delta T}\right)_{p}, \quad \cdot \quad \cdot \quad \cdot \quad \cdot
$$

and

$$
S_{\nu} m=S^{\prime}{ }_{p}, \quad \text {. . . . . . . }
$$


where $\left(\frac{\delta u_{a}}{\delta T}\right)_{D}=\left(\frac{\delta u_{a}}{\delta T}\right)$ for a perfect gas. It follows then that

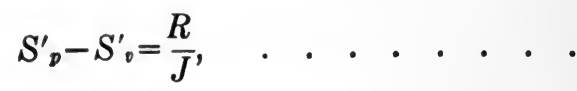

and

$$
\frac{S_{p}^{\prime}{ }_{p}}{S_{0}^{\prime}}=\frac{\frac{5 R}{2 J}+\left(\frac{\delta u_{a}}{\delta T}\right)_{0}}{\frac{3 R}{2 J}+\left(\frac{\delta u_{a}}{\delta T}\right)_{0}} . . . . .
$$

In the special case that $\left(\frac{\delta u_{a}}{\delta T}\right)_{0}=0$ the foregoing equation becomes $\frac{S_{p}^{\prime}}{S_{v}}=1.666$.

It will be instructive to consider the ratio of the specific heats for a number of gases at $0^{\circ} \mathrm{C}$. given in Table II. It

\begin{tabular}{|c|c|c|}
\hline Substance. & Formula. & $\frac{S_{p}^{\prime}}{S_{0}^{\prime}}$ \\
\hline Mercury .................. & $\mathrm{Hg}$ & 1.666 \\
\hline 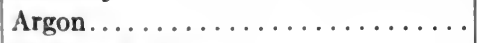 & A & 1.667 \\
\hline Carbon monoxide.............. & $\mathrm{CO}$ & 1.403 \\
\hline Carbon dioxide ............... & $\mathrm{CO}_{2}$ & 1.311 \\
\hline Ethelene................... & $\mathrm{C}_{2} \mathrm{H}_{4}$ & 1.245 \\
\hline Propane................... & $\mathrm{C}_{3} \mathrm{H}_{8}$ & 1.153 \\
\hline Methyl ether. . . . . . . . . . . . . & $\mathrm{C}_{2} \mathrm{H}_{6} \mathrm{O}$ & 1.107 \\
\hline Ethyl ether $\ldots \ldots \ldots \ldots \ldots \ldots$ & $\mathrm{C}_{4} \mathrm{H}_{10} \mathrm{O}$ & 1.097 \\
\hline
\end{tabular}
TABLE II

will be seen that for the mon-atomic gases argon and mercury $\cdot$ the ratio is practically the same as that given by the foregoing equation, and that therefore for these gases $\left(\frac{\delta u_{z}}{\delta T}\right)_{0}=0$, i.e., the kinetic energy of motion of translation only changes with change of temperature. This does not, however, hold 
when the molecules of the gas consist of two or more atoms, in a general way the deviation of this ratio from the value 1.666 increases with the complexity of the molecule. This is what we would expect since a change in temperature increases the violence of molecular collision through the increase of velocity of translation, which would give rise to an increase in the velocity of rotation of each molecule, changes of the atomic configuration through collision and increased velocity of rotation, etc.

It will be instructive to calculate the value of $\left(\frac{\delta u_{a}}{\delta T}\right)_{\text {. }}$ in the case of one of the complex gases-say ether. We have

and therefore

$$
1.097=\frac{\frac{5 R}{2 J}+\left(\frac{\delta u_{a}}{\delta T}\right)_{0}}{\frac{3 R}{2 J}+\left(\frac{\delta u_{a}}{\delta T}\right)_{0}}
$$

$$
\left(\frac{\delta u_{a}}{\delta T}\right)_{0}=\frac{17.6 R}{2 J}
$$

and thus the heat absorbed in changing the molecular internal energy is very much greater than the heat $3 R / 2 J$ absorbed in changing the kinetic energy of motion of translation, or in doing the external work $R / J$ on carrying out the heating at constant pressure. The values of $\left(\frac{\delta u_{a}}{\delta T}\right)_{0}$ thus vary very much with the complexity of the molecule.

The ratio of the two specific heats of a gas, it may be pointed out, can be determined with a much greater accuracy than either of these quantities separately from measurements of the velocity of sound $V_{s}$ which is given by the equation

$$
V_{s}=\sqrt{\frac{p}{\rho} \frac{S_{p}}{S_{0}}}
$$


where $p$ and $\rho$ denote the pressure and density of the gas. The value of $V_{s}$ for any given gas may be determined in the laboratory by measuring the wave length $\lambda$ in a Kundt's tube corresponding to a musical note of known frequency $n$, these quantities being connected by the equation $V_{s}=n \lambda$. It may be noted that since sound consists of the propagation of waves of compression and rarefraction, the velocity with which they travel is closely connected with the velocity of translation of the molecules.

The specific heat $S^{\prime}{ }_{v l}$ at eonstant volume per gram molecule of a liquid, or gas not obeying Boyle's law, is expressed by the equation

$$
S^{\prime}{ }_{\mathrm{rl}}=\left(\frac{\delta U_{e}}{\delta T}\right)_{0}+S_{{ }_{0}}^{\prime}, \quad \ldots \quad . \quad .
$$

where $U_{e}$ denotes the heat absorbed in internal energy changes on allowing the substances to expand at constant temperature till its volume is infinite. This equation may be proved by passing the substance through a cycle and equating to zero the internal work done. Thus on expanding the substance at constant temperature till its volume is infinite, and then raising the temperature of the resultant gas by $\delta T$ at constant volume, the internal work $U_{e}+S^{\prime}{ }_{0} \cdot \delta T$ is done. On compressing the substance to its original volume at constant temperature, and lowering its temperature by $\delta T$ at constant volume, the internal energy changes by $-U_{e}-\delta U_{e}-S_{r l}^{\prime} \cdot \delta T$. On equating the sum of the internal energies to zero equation (28) is obtained.

If the equation of state (Section 26) of the substance under consideration is known the value of $\left(\frac{\delta U_{e}}{\delta T}\right)_{0}$ can at once be calculated. According to thermodynamics

$$
\left(\frac{\delta U}{\delta v}\right)_{T}=T\left(\frac{\delta p}{\delta T}\right)_{v}-p
$$


where $U$ denotes the total internal energy of a substance of volume $v$, temperature $T$, and pressure $p$. On multiplying this equation by $\delta v$ and integrating it between the limits $v$ and infinity we obtain

$$
-U_{e}=U_{0}-U_{\infty}=\int_{v}^{\infty}\left\{T\left(\frac{\delta p}{\delta T}\right)_{v}-p\right\} \delta v .
$$

The right hand side of this equation may be evaluated by writing the equation of state in the form $p=\phi(T, v)$ and substituting for $p$.

Similarly it can be shown that the specific heat $S_{p l}^{\prime}$ per gram molecule at constant pressure is given by

$$
S_{p l}^{\prime}=\left(\frac{\delta U_{e}}{\delta T}\right)_{p}+S^{\prime}{ }_{v}+\frac{p}{J}\left(\frac{\delta v}{\delta T}\right)_{p} \cdot \quad \cdot
$$

The internal specific heat $S^{\prime}{ }_{i p l}$ per gram molecule at constant pressure is therefore given by

$$
S_{i p l}^{\prime}=\left(\frac{\delta U_{e}}{\delta T}\right)_{p}+S^{\prime}{ }_{v}, \quad \text {. . . . . . }
$$

which is $m$ times the specific heat $S_{g}$ per gram.

In the case that the molecules of a substance are partly dissociated the expressions for the two specific heats for the gaseous state will have to be modified. Consider a gram molecule of molecules containing $N$ molecules of which $N_{s}$ are dissociated, each into $q$ molecules. The kinetic energy term $\frac{3 R}{2 J}$ has then to be replaced by the term

$$
\frac{3 R}{2 J} \cdot \frac{\delta}{\delta T}\left(1+\frac{N_{s} q}{N}\right) T \text {. }
$$

The term $\left(\frac{\delta u_{a}}{\delta T}\right)_{0}$ has to be replaced by the term

$$
\delta T\left(u_{a}-\frac{H N_{s}}{N}\right)
$$


where $H$ denotes the heat of formation of a gram molecule of molecules. Equations (22) and (24) are accordingly replaced by the equation

$$
\begin{aligned}
S^{\prime}=\frac{3 R}{2 J N}\{N & \left.+q\left(N_{s}+T\left(\frac{\delta N_{s}}{\delta T}\right)_{0}\right)\right\} \\
& +\left(\frac{\delta u_{a}}{\delta T}\right)_{0}-\frac{N_{s}}{N}\left(\frac{\delta H}{\delta T}\right)_{0}-\frac{H}{N}\left(\frac{\delta N_{s}}{\delta T}\right)_{0} \ldots . .
\end{aligned}
$$

and the equation obtained by substituting $S^{\prime}{ }_{p},\left(\frac{\delta N_{s}}{\delta T}\right)_{p}$, for $S^{\prime},\left(\frac{\delta N_{8}}{\delta T}\right)_{0}$ in the foregoing equation, and adding the $\operatorname{term} \frac{p}{J}\left(\frac{\delta v}{\delta T}\right)_{p}$.

In the case of a mixture of substances it is useful to define the quantity partial specific heat. Thus if $S_{e r}$ denotes the internal heat capacity at constant pressure of a mixture of molecules $e$ and $r$, it may be written

$$
S_{e r}=S_{e}+S_{r}, \quad \cdot \quad \cdot \quad \cdot \quad \cdot \quad \text { : }
$$

where $S_{e}$ denotes the internal specific heat associated with the molecules $e$, and $S_{r}$ that associated with the molecules $r$. This equation may also be written

$$
S_{e r}=N^{\prime}{ }_{r} s_{r}+N^{\prime}{ }_{e} s_{e}, \quad . \quad . \quad . .
$$

where $N^{\prime}{ }_{r}$ and $N^{\prime}{ }_{e}$ denote respectively the numbers of molecules $r$ and $e$ in the mixture, and $s_{r}$ and $s_{e}$ the specific heats of a molecule $r$ and of a molecule $e$ respectively. The change in heat capacity that the mixture undergoes on adding a small number of molecules-say of $r$, is expressed by differentiating the foregoing equation with respect to $N^{\prime}$, giving

$$
\left(\frac{\delta S_{e r}}{\delta N_{r}^{\prime}}\right)_{D}=s_{r}+N_{r}^{\prime}\left(\frac{\delta s_{r}}{\delta N_{r}^{\prime}}\right)_{D}+N^{\prime}\left(\frac{\delta s_{e}}{\delta N_{r}^{\prime}}\right)_{D}
$$


Now the value of $s_{r}$ of a molecule $r$ depends on the nature and relative positions of the surrounding molecules, and the same holds for $s_{e}$. These conditions will evidently be altered to a vanishing extent by the addition of a small number of molecules $r$ to the mixture, and therefore very approximately $\left(\frac{\delta s_{r}}{\delta N_{r}^{\prime}}\right)_{p}$ and $\left(\frac{\delta s_{e}}{\delta N_{r}^{\prime}}\right)_{p}$ are equal to zero. The foregoing equation then gives

$$
s_{r}=\left(\frac{\delta S_{e r}}{\delta N_{r}^{\prime}}\right)_{p}, \quad \cdot \quad \cdot \quad \cdot \quad \cdot \quad \cdot
$$

and thus $s_{r}$ can be determined by measuring $\left(\frac{\delta S_{e r}}{\delta N_{r}^{\prime}}\right)_{p}$. The value of $s_{e}$ may now be determined from equation (33), or in the same way as $s_{r}$. In the latter case the values of $s_{r}$ and $s_{e}$ obtained should make equation (33) vanish, and in this way therefore the accuracy of the determinations may be tested.

It may be mentioned here that the matter in Sections 30 and 39 , has a bearing on specific heats.

The quantity $U_{e}$ in the foregoing equations has in practice a finite value depending on the temperature, unless the substance behaves as a perfect gas, in which case the quantity is zero. It appears, therefore, that on changing the temperature of a substance not obeying the gas laws, a part only of the heat energy absorbed is converted into kinetic energy of translation of the molecules. The remaining part is expended in other ways, most probably in overcoming the attraction between the molecules, evidence of the existence of which will now be considered.

14. Evidence that Molecules and Atoms are surrounded by Fields of Force.

There is a good deal of evidence that molecules and atoms are surrounded by strong fields of force which decrease 
rapidly in intensity from the center outwards. The internal heat of evaporation $L$ of a liquid, for example, represents almost entircly work done against these forces during the separation of the molecules. We may write accordingly

$$
L=\left(U_{1}-U_{2}\right)+\left(u_{1}-u_{a}\right),
$$

where $U_{1}-U_{2}$ denotes the change in potential energy of the liquid during evaporation due to the molecular attraction, and $u_{1}-u_{a}$ the change in internal energy of the molecules. The free kinetic energy of the molecules is not altered during the process of separation according to Section 21, and hence $u_{1}-u_{a}$ represents the change in molecular energy due to changes in atomic configuration and velocity of molecular rotation. It is likely to be small in comparison with $U_{1}-U_{2}$, the work done against molecular attraction.

When the volume of a dense gas not obeying Boyle's law is increased at constant temperature, a larger amount of heat is absorbed than corresponds to the external work done. The explanation is the same as in the case of the evaporation of a liquid.

The forces in question are appreciable over much greater distances of separation of the attracting molecules than of the order of the distance of separation in the liquid state, i.e., $10^{-8} \mathrm{~cm}$. This is shown by the Joule-Thomson effect. On passing a stream of gas at a volume $v$ per gram and pressure $p$ through a porous plug on the other side of which the gas has a volume $v^{\prime}$ and pressure $p^{\prime}$ the temperature of the stream emerging from the plug is not the same as that entering it. If the gas does not obey Boyle's law $p^{\prime} v^{\prime}$ is not equal to $p v$, and a part of the temperature change is accounted for by the external work done in the process, which is equal to $p^{\prime} v^{\prime}-p v$. This can evidently at once be calculated. The other part of the temperature change is accounted for by the work necessary to separate the molecules against their molecular forces during their passage 
through the porous plug, which is done at the expense of their kinetic or heat energy. The temperature change is usually negative, but it may also be positive, depending on the values of $p, p^{\prime}, v$, and $v^{\prime}$. In the first case energy is required to separate the molecules, and hence attraction is the paramount force, while in the second case work is done on the molecules, and the paramount force is therefore repulsion. The change in internal molecular energy during the process, represented by $u_{1}-u_{a}$, is probably negligible. Thus we see that forces of attraction and repulsion exist between molecules whose relative magnitudes depend upon the distances of separation; and the resultant force may therefore be represented by the sum of at least two terms, one representing attraction and the other repulsion, the value of the attraction term decreasing according to the nature of the internal heat of evaporation of a liquid and the foregoing experiments more rapidly with the distance of separation of the molecules than the repulsion term.

At the absolute zero of temperature the molecules of a substance have no kinetic energy of translation. It is fairly certain that the volume of the substance under those conditions is not zero. There exists therefore very probably another repulsion term in the law of force counteracting the attraction term for very small distances of separation of the molecules, in which case the apparent volume of the substance would not vanish at the absolute zero.

The considerations in the foregoing paragraph discard the notion of molecular volume. It is important in this connection to note that the volume associated with matter manifests itself to us only by resistance to force; and molecules may therefore be, and probably ought to be, simply regarded as centers of force. The fact that the apparent volume of a molecule depends on external conditions such as the temperature, etc., appears to show that this view ought to be taken. (See Sections 19, and 24.) 
It follows from the foregoing considerations that as a first approximation the force of attraction between two molecules may be represented by an expression consisting of the sum of three terms, one of which has a positive and the remaining two negative signs. The numerical value of one of the negative terms decreases more rapidly with the distance of separation of the molecules than the value of the positive term, while the value of the other negative term decreases less rapidly. The value of the last-mentioned term will thus be predominant for large distances of separation of the molecules, giving rise to a repulsion between them which shows itself as a heating effect in Joule-Thomson experiments. The value of the other negative term will be predominant for close distances of approach of the molecules, giving rise to a repulsion between them which shows itself as an apparent volume of the molecules. For intermediate distances of separation of the molecules the positive term will be predominant and the force one of attraction.

It is obvious that the effect of the molecular forces on the properties of a substance as a whole arises througn their effect on the relative motion of the molecules. This leads us to consider the influence of one molecule upon the motion of another in a substance.

\section{Molecular Interaction.}

In treatises on the Kinetic Theory of Gases molecules are usually supposed to consist of perfectly elastic spheres having no fields of force surrounding them. The interaction of the molecules would then consist simply of collisions. This is no doubt far from being the case in practice, according to the previous Section. If molecules and atoms consist, simply of centers of force it would be hard, if not impossible, to define what is meant by a collision. The mathematical 
investigations based on molecular collision may therefore lead to results having little to do with the actual facts. It appears therefore that the proper development of the subject should be along lines which do not involve molecular collision.

This will be emphasized by a consideration of the general nature of the interaction of two molecules when the law of force of interaction is of the nature described in the previous Section. This is graphically shown in Fig. 3, which in a general way shows the paths described by a molecule having a high velocity in approaching a molecule at rest, correspond-

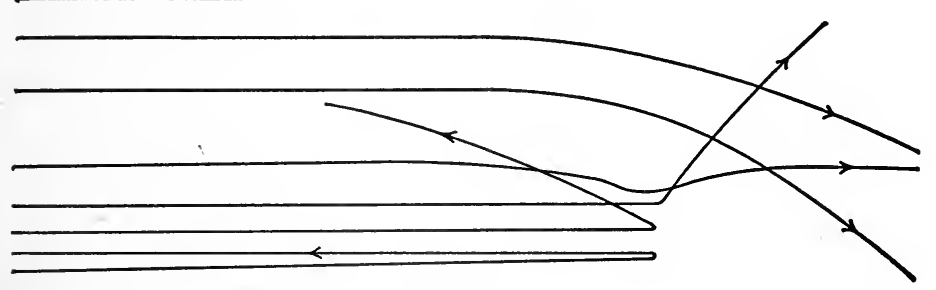

FIG. 3.

ing to different distances of the molecule at rest from the line of prolongation of the initial direction of the moving molecule. For small values of these distances the path of the molecule will be mainly affected by the repulsion existing on close approach; for greater distances the path is mainly affected by forces of attraction, which deflects the moving particle towards the one at rest; for still greater distances a slight repulsion between the molecules tends to deflect the moving molecule in the opposite direction. When both molecules are moving their paths are similar to the foregoing, the molecules then simultaneously approach or recede from each other, or move in the same direction.

The nature of the path of a molecule, it will be seen, 
depends considerably on the initial direction of motion of the molecule, and in each case is complex. In practice each path would probably be much more complex than indicated by Fig. 3, which does not pretend to exhibit the actual state of affairs that exists in practice, but has been drawn to give the reader merely some idea of the complexity of the motion of a molecule under the influence of another molecule. It is evident, therefore, that the effect of the molecular forces, in whatever connection, could scarcely be represented by the effect of the collision of elastic spheres.

In the case of a liquid the paths of two molecules under each other's influence would be affected by the vicinity of other molecules, and would therefore be very much more complicated than those indicated by Fig. 3 . 


\section{CHAPTER II}

THE EFFECT OF THE MOLECULAR FORCES ON THE DYNAMICAL PROPERTIES OF A MOLECULE IN A DEN̦SE GAS OR LIQUID

16. The Velocity of Translation of a Molecule in a Liquid or Dense Gas when passing through a Point at which the Forces of the Surrounding Molecules neutralize each other.

It is often assumed, without any apparent reason, that the average velocity of a molecule in a liquid or dense gas is the same as that it would have in the gaseous state at the same temperature. But this is not likely to hold on account of the interaction of the molecules due to the existence of molecular forces. Information in this matter is obtained from considering what is meant by a thermometer indicating the temperature of a gas in which it is placed.* From the Kinetic Theory it follows that a thermometer placed in a gas assumes its temperature through being bombarded by the gas molecules. The temperature indicated does not depend on the number of molecules falling per square $\mathrm{cm}$. per second on the surface of the thermometer bulb, but only on the average kinetic energy of a molecule in the gas according to Section 8 . The velocity with which a molecule actually strikes the bulk depends, however, on the attraction exerted by its material. Therefore, if the bulb is covered with a layer of material of much greater density

* R. D. Kleeman, Phil. Mag., July 1912, pp. 100-102. 
than the bulb itself the velocity of impact may be very much increased. But the temperature indicated by the thermometer is not altered thereby, as we know from experiment. Thus the temperature indicated corresponds to the kinetic energy of a molecule when not under the attraction of the thermometer bulb, or the additional velocity given to the molecule by the attraction of the bulb has no effect on the temperature indicated.

Again, if a very dense solid were placed somewhere in the gas, the molecules in its zone of attraction would possess a greater velocity of translation than outside the zone. The temperature indicated by the thermometer would, however, not be altered thereby, but would correspond to the kinetic energy of the motion of translation of the gas molecules outside this zone, and the zone of attraction of the thermometer bulb, i.e., to the kinetic energy when the molecules are not under the action of any forces.

The same result will also hold in the case of a liquid, or dense gas, in which 'case the temperature indicated by the thermometer corresponds to the velocity each molecule has when not under the action of a force, which occurs when passing through a point where the forces of the surrounding molecules neutralize each other. There are evidently numbers of such points in a liquid which change their positions with the motion of the molecules. The velocity which a molecule has when passing through such a point is not necessarily always the same, but an average velocity may be associated with it. When the molecule is not passing through such a point in a dense gas or liquid it is under the action of forces, and its velocity on the average is then greater (Section 17) than the foregoing average velocity. Therefore the average velocity of a molecule when not under the action of forces may be called its average minimum velocity.

It is evident then that if we consider two masses of the 
same substance, one in the perfectly gaseous state and the other in the liquid state, and they possess the same temperature, the average kinetic energy of a gas molecule is the same as the average minimum kinetic energy of a molecule in the liquid state, in other words, the average kinetic energy of a molecule when not under the action of a force in a liquid or. dense gas is the same as the average kinetic energy it would have in the perfect gaseous state at the same temperature.

We may also reason in a reverse manner. A thermometer placed in a liquid indicates its temperature through being bombarded by the liquid molecules. The velocity of impact and the general nature of the bombardment may be changed without changing the temperature indicated by changing the nature of the thermometer bulb, or compressing the liquid at constant temperature. The question then arises: what is the connection between the temperature indicated and the velocity of translation of a molecule under stated conditions in the liquid? Now the velocity of a molecule that means anything definite in a liquid corresponds to its independent velocity, or the velocity when not under the action of an external force, and we must therefore endeavor to connect this velocity with the temperature indicated. Now in the case of a gas a thermometer indicates the temperature corresponding to the kinetic energy of a molecule when not under the action of a force. There is no reason whatever why this should not hold in the case of a liquid. We conclude, therefore, that the average kinetic energy of a molecule in a liquid under these conditions is the same as in the gaseous state at the same temperature.

This result may be established in a somewhat different way. Consider two chambers having a wall in common filled with gases at the same temperature. Suppose that one-half of the common wall adjacent to one of the gases is replaced by a much denser material so that the molecules strike the 
wall on that side with a greater velocity than on the other side. This change in the nature of one side of the wall will not give rise to a flow of heat from one side to the other, since this would be contrary to the laws of thermodynamics. The heat effect produced by the molecules on impinging on the wall therefore does not depend on the velocity of impact, but on the velocity when not under the action of the attraction of the wall. The foregoing result follows then in a similar way as before.

We will now turn our attention to the velocity of a molecule at other points in a liquid than those considered.

\section{The Total Average Velocity of Translation} of a Molecule in a Substance.

When a molecule is not passing through a point in a dense substance at which the forces of the surrounding molecules neutralize each other, its velocity is likely to differ, on account of the effect of molecular attraction and repulsion, from its velocity at such a point, which we have seen in the previous Section is the same as that it would have in the perfectly gaseous state at the same temperature. The total average velocity of the molecule, corresponding to the complete path desoribed during an infinitely long time, is therefore likely to differ from the velocity defined as the average minimum velocity. When a substance is in the perfectly gaseous state these two velocities are evidently very approximately equal to one another, or denote approximately the same thing. But evidently this cannot be the case when the density of the substance is such that the motion of the molecule is constantly influenced by the attraction and repulsion of the surrounding molecules. In fact, it can be shown* that in the case of a liquid the total average

* R. D. Kleeman, Phil. Mag., July, 1912, pp. 101-103. 
velocity is several times that of the average minimum velocity.

Suppose that the volume of a gram of liquid is changed by a small amount at a low temperature in contact with its saturated vapor by changing the temperature. The energy spent per molecule in overcoming the molecular attraction is equal to $m_{a} \cdot d L$, where $L$ denotes the internal heat of evaporation in ergs per gram and $m_{a}$ the absolute molecular weight of a molecule. The average force acting on a molecule during the expansion is therefore $\frac{d L}{d x} m_{a}$, where $x$ denotes the distance of separation of the molecules. Now $x=\left(\frac{m_{a}}{\rho}\right)^{1 / 3}$, where $\rho$ denotes the density of the liquid, and the force may therefore be written $3 m_{a}{ }^{2 / 3} \rho^{4 / 3} \frac{d L}{\delta \rho}$. We may assume without any serious error that on the average the expenditure of energy on a molecule during its motion of translation is proportional to the distance traversed, and the force acting equal to the foregoing value. Therefore, when a molecule traverses a distance equal to half the distance of separation of the molecules it may on the average gain or lose the amount of energy $\frac{3}{2} m_{a} \rho \frac{d L}{d \rho}$, which corresponds to a change in velocity in cms. equal to $\sqrt{3 \rho \frac{d L}{\delta \rho}}$. For example, in the case of ether at $0^{\circ} \mathrm{C}, \rho=.7362$, and $\frac{d L}{\delta \rho}=\frac{2.72 \times 4.18 \times 10^{7}}{.0014}$ corresponding to a change of $10^{\circ} \mathrm{C}$., which gives a change in velocity equal to $1.69 \times 10^{5} \mathrm{cms}$. $/ \mathrm{sec}$. of the molecule. The average minimum velocity of a molecule, which corresponds to that of a molecule in the gaseous state at the same temperature, is equal to $3.03 \times 10^{4} \mathrm{cms}$. $/ \mathrm{sec}$. We see therefore that the total average velocity of a molecule in a liquid may be several times that corresponding to its temperature. 
It will be convenient now to connect this velocity by a general equation with a quantity which has already been discussed in connection with gases.

18. The Number of Molecules crossing a Square Cm. in a Substance of any Density from one Side to the Other per Second.

If the molecules consisted in three equal streams moving at right angles to each other, and a plane one square $\mathrm{cm}$. in area were taken at right angles to one of the streams, $N_{c} V_{t}(2 \times 3)$ molecules would cross it in one direction per second, and the same number in the opposite direction, where $N_{c}$ denotes the number of molecules per cubic cm., and $V_{t}$ the total average velocity. This expression may be obtained and along the same lines as the similar expression in equation (17). If, however, the molecules move in all directions, as is actually the case, the number of molecules $n$ crossing a square $\mathrm{cm}$. from one side to the other in all directions is double the above number, that is

$$
n=\frac{N_{c} V_{t}}{3} \text {. . . . . . }
$$

This may be proved by means of the geometrical considerations used in Section 11. The result may, however, be at once deduced from the results obtained in that Section. Thus it was shown that the pressure of a gas may be written

$$
p=n_{0} A=n \frac{A}{2},
$$

where $n_{o}$ denotes, if the molecules consist of three equal streams at right angles, the number crossing a square $\mathrm{cm}$. taken at right angles to one of the streams, and $n$ denotes the number of molecules when they move in all directions, as is the case in practice. Hence it follows that $n=2 n_{0}$, 
the result just used, which obviously does not hold only for a gas, but in general.

A molecule probably does not consist of a point in space, but possesses a real or apparent molecular volume, which influences its motion and consequently the properties of the substance of which it forms part. This effect will be first considered in connection with a gas.

\section{The Effect of Molecular Volume on $p$ and} $n$ in the Case of an Imperfect Gas.

We have seen in Section 6 that the effect of the volume of the molecules of a substance is to increase its external pressure from that it would have if the molecules possessed no volume. The effect of a molecular volume is evidently to decrease the space available for molecular motion of translation. It is therefore equivalent to supposing that the molecules are devoid of volume, and that the volume of the substance (as a whole) is decreased by an amount $b$, as is shown by means of a diagram in Fig. 4. This quantity is the apparent volume of the molecules connected with molecular motion. The external pressure $p$ in this case might thus be written

$$
p=\frac{R T}{v-b}, . \quad . \quad . \quad . \quad .
$$

in conformity with the equation $p=R T / v$ of a perfect gas, where $v$ refers to a gram molecule of molecules.

It will not be difficult to see that this result will also hold if the apparent volume of the molecules is caused by molecular forces of repulsion, which do not permit an approach of two molecules beyond a certain distance.

If the molecules of a gas consist of a number of hard elastic spheres not surrounded by fields of force, their volume would not interfere with their velocity of translation, 
which would simply correspond to the temperature of the gas. This will be made clear by an inspection of Fig. 4 according to which a set of molecules possessing molecular volume of the foregoing kind move in the space $v$ with the same velocity.as if they were devoid of volume and moved in the space $v-b$. Equality of velocities is necessary in the two cases since the external pressures are the same. Since the molecular forces may give rise to an apparent volume of the molecules as well as affect their velocity of

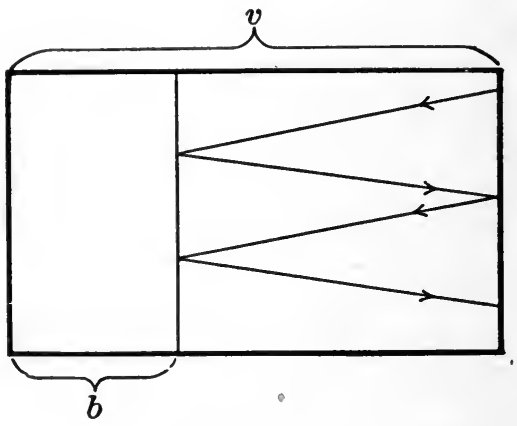

Fig. 4.

translation, the foregoing considerations suggest a definition of molecular volume which is quite definite and applies to all states of matter. Thus the apparent molecular volume of a substance may be defined as the quantity associated with the molecules whose magnitude affects the external pressure of the substance at constant temperature and volume, but does not affect the average velocity of translation of the molecules. The latter property is expressed by the equation

$$
\left(\frac{\delta V_{t}}{\delta b}\right)_{T, 0}=0
$$


where $V_{t}$ denotes the average velocity of translation, and $b$ the apparent volume of the molecules. The velocity $V$, is a function of the temperature and therefore

$$
\left(\frac{\delta V_{t}}{\delta T}\right)_{v}=\left(\frac{\delta V_{t}}{\delta b}\right)_{T, v}\left(\frac{\delta b}{\delta T}\right)_{0}+\left(\frac{\delta V_{t}}{\delta T}\right)_{b, v}=\left(\frac{\delta V_{t}}{\delta T}\right)_{b, v},
$$

by the preceding equation. It is also a function of the volume and therefore

$$
\left(\frac{\delta V_{t}}{\delta v}\right)_{T}=\left(\frac{\delta V_{t}}{\delta b}\right)_{T, 0}\left(\frac{\delta b}{\delta v}\right)_{T}+\left(\frac{\delta V_{t}}{\delta v}\right)_{T, b}=\left(\frac{\delta V_{t}}{\delta v}\right)_{T, b} .
$$

These equations express the relations between $V_{t}$ and $b$ according to the above definition of $b$.

It should be carefully noted that $b$ is a perfectly definite mathematical quantity, though there might be some difficulties in defining the exact nature and geometrical configuration of the real, or apparent, volume of a single molecule.

The apparent volume of a molecule, whatever its cause, is likely to change little if at all with the density of the substance. The value of $b$ may therefore without the risk of introducing any serious error be taken constant over small changes in density. This is very useful in the determination of the values of $b$ from simultaneous equations, as is carried out in Section 29.

The value of $b$ would vary with the temperature if caused by forces of repulsion between the molecules. Thus two molecules moving towards each other in a substance along the same straight line continue approaching each other until their kinetic energy of translation is completely transformed into potential energy of repulsion, after which they retrace their path. Hence a nearer approach must take place with an increase of temperature, which corresponds to an increase in kinetic energy, and a corresponding decrease in the value of $b$ would result. 
It will be evident on reflection that the forces of attraction and repulsion between two molecules might be changed at constant temperature and volume so that $V_{\imath}$ remains the same, in which case a change in $b$ may result, or so changed that $b$ remains the same, in which case a change in $V_{t}$ may result. This shows that the definition of $b$ given is admissible.

A change in temperature or density of a substance may evidently result in a change of both $b$ and $V_{t}$. The foregoing equations then express that whether or no $b$ changes, the changes in $V_{\imath}$ are the same as if $b$ remained constant.

It can be shown that $n$, the number of molecules passing through a square $\mathrm{cm}$. in one direction per second, is independent of the apparent volume of the molecules, since the velocity of translation is also independent of this quantity. Let us suppose that we are initially dealing with molecules devoid of volume, and to which is then given an apparent volume $b$. We may then suppose that the resultant molecules possess no volume and occupy the volume $v-b$ of the volume $v$ for motion of translation. If $N_{c}$ denote the number of molecules per cubic $\mathrm{cm}$. when the gas occupies the volume $v$, the number when it occupies the volume $v-b$ is $N_{c} v /(v-b)$. The number of molecules crossing a square $\mathrm{cm}$. per second is accordingly changed from $n$ to $n v /(v-b)$, since the velocity of translation remains unaltered, where $n$ corresponds to the molecules having no volume and occupying as a whole the volume $v$. But in practice the molecules with their apparent volumes would be distributed throughout the volume $v$, instead of being separated from their volumes as shown in Fig. 4, and the number of molecules crossing a square $\mathrm{cm}$. per second is therefore equal to the foregoing expression reduced in the ratio of $v-b$ to $v$, which makes it equal to $n$, the value when the molecules have no volume.

This result ean be very simply deduced from equation (35). If the apparent volume $b$ of the molecules is changed 
without changing $V_{t}$, by definition $n$ remains constant according to this equation, and it is thus independent of the volume of the molecules. This method of obtaining the result is perhaps not so instructive as the preceding one. The result is mathematically expressed by the equation

$$
\left(\frac{\delta n}{\delta b}\right)_{T, 0}=0 .
$$

By means of this equation and the Differential Calculus it can be shown, similarly as in connection with the quantity $V_{t}$, that

$$
\left(\frac{\delta n}{\delta T}\right)_{v}=\left(\frac{\delta n}{\delta T}\right)_{v, b}, \quad \text { and } \quad\left(\frac{\delta n}{\delta v}\right)_{T}=\left(\frac{\delta n}{\delta v}\right)_{T, b} \cdot
$$

These equations express the relations between $n$ and $b$ according to its definition.

If the molecules of a gas were devoid of volume and molecular forces the laws of a perfect gas apply, and the external pressure $p$ of the gas may then be written

$$
p=n \frac{A}{2},
$$

according to equation (20) in Section 11.

The acquisition of an apparent volume $b$ by the molecules does not change $n$, we have seen, but it changes the external pressure in the ratio of $v$ to $v-b$. Hence the external pressure of a gas under these conditions may be written

$$
p=\frac{v}{v-b} n \frac{A}{2} \text {. }
$$

Since $n$ is defined and used in connection with the pressure produced by the molecules of a substance, the crossing of a plane by a molecule, if the molecules are devoid of volume, is obviously associated with the crossing of its 
center of mass. This also holds when the molecules possess volume, according to the method the subject has been developed.

The external pressure of a gas is equal to the expansion pressure due to the motion of translation of the molecules tending to expand the gas. But in the case of a liquid or dense gas this does not hold, due to the effect of the forces of attraction and repulsion between the molecules, and the expansion pressure of a dense substance is therefore a quantity requiring special consideration.

20. The Expansion Pressure exerted by the Molecules of a Substance of any Density.

The pressure exerted by a gas in a closed vessel upon its walls is, according to the Kinetic Theory of Gases, due to the change in momentum the molecules undergo through collision with the vessel's walls. The velocity of translation of the molecules, the density and external pressure of the gas, are then connected by equation (7) in Section 6 . This equation holds, however, strictly only if the walls of the vessel do not exert any attraction upon the molecules. It holds very approximately in the cases usually occurring in practice, in which the size of the vessel is so large that the greater mass of the gas is only slightly under the influence of the attraction of the walls. But under certain conditions this influence may be so large that the equation cited does not hold. It will be of importance to investigate this effect of molecular forces more closely, as it has a bearing on the kinetic properties of liquids. Thus let $A B$ and $C D$, in Fig. 5 , represent two opposite walls of a vessel, and let the planes $A^{\prime} B^{\prime}$ and $C^{\prime} D^{\prime}$ denote the boundaries of the zones in which the attraction of the walls is of appreciable magnitude. A molecule on entering onc of the zones has its velocity increased through the attraction of the material of the 
adjacent wall. Thus the molecule would exert a pull upon the wall during the whole time it is in the zone. Now it follows from the dynamical equation $F t=m_{a} V$ that the average pull per unit time exerted by the molecule during the time it is in the zone, is balanced by the average thrust acting in the opposite direction upon the wall during that period, due to the reversal of the direction of the additional momentum acquired by the molecule in the zone. The force exerted by the molecule upon the wall during one rebound is thus not affected by molecular attraction. The

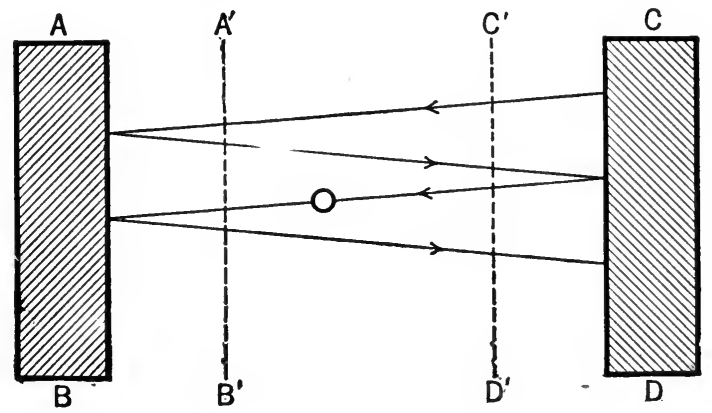

FIG. 5 .

total pressure exerted by the molecules of the vessel on the wall would therefore depend only on the number impinging per square $\mathrm{cm}$. per second on the wall. This number is equal to the number of molecules entering a zone per square cm. per second. It evidently depends upon the velocity of translation of the molecules outside the zones, and the number of molecules per cubic $\mathrm{cm}$. in that region. If the thickness of each zone is small in comparison with the diameter of the vessel, the average velocity of translation of the molecules may be taken equal to their velocity outside their zones.

If, however, the thickness of the zone is comparable with the diameter of the vessel this no longer holds. Thus 
suppose that the walls $A B$ and $C D$ are so close to one another that the zones of attraction of the walls overlap as is shown in Fig. 6. The attraction exerted by one of the walls would be neutralized by that of the other wall in a plane $E F$ which lies midway between the walls. The velocity of a molecule in that plane would therefore be the same as that it would have between the planes $A^{\prime} B^{\prime}$ and $C^{\prime} D^{\prime}$ in Fig. 5. For according to Section 16 the velocity of translation of a molecule at a point where the various forces neutralize each

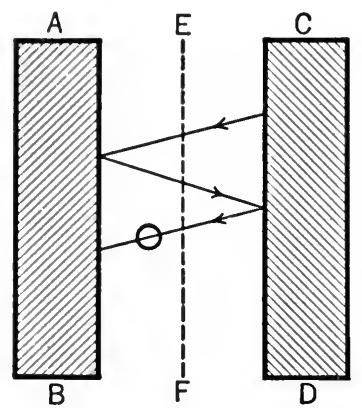

Fig. 6. other, is, at constant temperature, the same as its velocity when under the action of no force. The velocity for all points outside of the plane $E F$, would, however, be considerably greater than that in the plane. The latter velocity would therefore be considerably smaller than the total average velocity. As before the attraction of the walls would not affect the force exerted by a molecule corresponding to a single rebound. But it would evidently affect the number of times a molecule crosses from one wall to the other per second. Therefore the pressure the molecules would exert upon the walls $A B$ and $C D$ under the conditions shown in Fig. 6 would indirectly be increased by the molecular attraction of the walls. The pressure per square $\mathrm{cm}$. on each wall would accordingly be equal to $n A / 2$, where $n$ denotes the number of molecules crossing a square $\mathrm{cm}$. per second of the plane $E F$ from one side to the other-this quantity being affected by the attraction of the walls on the molecules, and $A / 2$ denotes the force exerted by a single molecule on rebounding from one of the walls, this quantity having the same value as when the walls possess no attrac- 
tion, its value having been determined in Section 11. A similar state of affairs exists in a liquid, or in a substance which does not behave as a perfect gas, the attraction between the molecules taking the place of the attraction of the foregoing walls.

These deductions will now be used to find an expression for the expansion pressure $P_{e}$ of a substance tending to expand it, due to the motion of translation of the molecules. The number of molecules $n$ crossing a plane one square $\mathrm{cm}$. in area in all directions from one side to the other, is equal to the number crossing in the opposite direction. We may therefore suppose, if the molecules possess no volume, that each stream of molecules is reflected from the foregoing plane and thus exerts a pressure upon it. Hence the expansion pressure $P_{e}$ would be equal to the number of molecules crossing the plane in one direction multiplied by the factor $A / 2$, which expresses the force exerted by a single molecule, which factor is the same as applies in the case of a perfect gas according to the preceding investigation, that is, under these conditions,

$$
P_{e}=n \frac{A}{2} \text {. }
$$

But if each molecule has an apparent volume it may exert a pressure across the plane without crossing it, and the foregoing formula has to be modified accordingly. We have seen in Section 19 that the molecular volume does not affect the value of $n$, but increases the expansion pressure in the ratio of $v$ to $v-b$. Hence in general the expansion pressure is given by

$$
P_{e}=n \frac{v}{v-b} \frac{A}{2}=n \frac{v}{v-b} 2.543 \times 10^{-20} \sqrt{\overline{T m}}, .
$$

where $v$ denotes the volume of a gram molecule, and $b$ 
the molecular volume, the value of $A$ being given by equation (19). This equation may be written

$$
P_{e}=\frac{v}{v-b} \frac{V_{t} N_{c}}{3} \frac{A}{2}=8.48 \times 10^{-21} \frac{v N_{c} V_{t}}{v-b} \sqrt{T m}, .
$$

by means of equation (35), where $V_{\imath}$ denotes the total average velocity of a molecule, and $N_{c}$ the molecular concentration. Since $V=\sqrt{\frac{3 R T}{m}}$, and $\frac{A}{2}=m_{a} \sqrt{\frac{3 R T}{m}} \quad$ (Sections 6 and 11), the foregoing equation may be written by the help of the equation $v N_{c} m_{a}=m$,

$$
P_{e}=\frac{V_{t}}{V} \frac{R T}{v-b}, \quad . \quad . \quad . \quad . \quad .
$$

where $V$ denotes the average kinetic energy velocity of a molecule, and $b$ is taken to refer to a gram molecule or molecules.

The foregoing equations are not corrected for the distribution of molecular velocities in the gaseous state, since the function $A$ in equation (38), from which the other equations are derived, has not been thus corrected. If this correction is carried out according to Maxwell's law the right hand side of each equation has to be multiplied by $\left(\sqrt{\frac{3 \pi}{8}}\right)$, or 1.085 , according to Section 11. It may be noted that if the kinetic energy velocity $V$ of a molecule in the gaseous state is eliminated from equation (40) by means of the equation $V_{a}=.922 \mathrm{~V}$ given in Section 8 , and the equation is corrected for Maxwell's law, an equation is obtained in which $V$ is replaced by $V_{a}$ the total average velocity of a molecule in the gaseous state. The equation in this form is independent of the distribution of molecular velocities when applied to the gaseous state, since then $V_{t}=V_{a}$.

If a substance behaved as a perfect gas at all densities 
we would have $V_{t}=V_{a}$ and $b=0$. The expansion pressure is then equal to the external pressure, according to equation (40) in the corrected form where $V_{a}$ takes the place of $V$, and the gas equation. But since this is not the case in practice, for $V_{t}>V_{a}$ according to Section 17, and $b$ is not zero according to Section 29, the expansion pressure is greater than the pressure $p=R T / v$. Hence there m' $1 \mathrm{~s}^{t}$, exist a negative pressure acting in the opposite direction to the external pressure to ensure equilibrium of the súbstance, since in practice the external pressure is usually less than $R T / v$. This negative pressure is called the intrinsic pressure and is discussed in the next Section.

The foregoing results may be extended to a mixture of molecules. Let us consider a mixture of two different molecules $e$ and $r$. Since the expansion pressure of a substance is caused by the motion of translation of the molecules, and each molecule produces pressure independently, the total expansion pressure is equal to the sum of the expansion pressures exerted by the molecules $e$ and $r$. The expansion pressure exerted by the molecules $e$ is evidently equal to

$$
n_{e} \frac{v_{e}}{v_{e}-b_{e}^{\prime}} 2.543 \times 10^{-20} \sqrt{T m_{e}},
$$

where $n_{e}$ denotes similarly as before the number of molecules $e$ crossing a square $\mathrm{cm}$. from one side to the other per second, $m_{e}$ denotes the relative molecular weight of a molecule, $v_{e}$ may be taken to refer to the volume of the mixture containing a gram molecule of molecules $e$, and $b^{\prime}{ }_{e}$ denotes the apparent volume in the volume $v_{c}$ which the molecules $e$ and $r$ appear to possess in obstructing the motion of a molecule $e$. Similarly the expansion pressure exprted by the molecules $r$ is equal to

$$
n_{r} \frac{v_{r}}{v_{r}-b_{r}^{\prime}} 2.543 \times 10^{-20} \sqrt{T m_{r}}
$$


where the symbols, $v_{r}, n_{r}, m_{r}$, and $b_{r}^{\prime}$, have meanings similar to the symbols, $v_{e}, n_{e}, m_{e}$, and $b_{e}^{\prime}$. The total expansion pressure is therefore given by

$$
P_{e}=2.543 \times 10^{-20}\left\{\frac{n_{e} v_{e}}{v_{e}-b^{\prime}{ }_{e}} \sqrt{T m_{e}}+\frac{n_{r} v_{r}}{v_{r}-b_{r}^{\prime}} \sqrt{T m_{\epsilon}}\right\},
$$

and its value in the case of a mixture of any number of different kinds of molecules is therefore given by

$$
P_{e}=2.543 \times 10^{-20} \sum \frac{n v}{v-b} \sqrt{T m} . . .
$$

Similarly corresponding to equations (39) and (40) we have

$$
P_{e}=8.48 \times 10^{-21} \sum \frac{v N_{c} V_{t}}{v-b} \sqrt{T m}, . .
$$

and

$$
P_{e}=\sum \frac{V_{t}}{V} \frac{R T}{v-b}
$$

for any number of constituents in the mixture. The foregoing equations may be corrected for Maxwell's distribution law similarly as before.

Approximate values of $b^{\prime}{ }_{e}$ and $b^{\prime}{ }_{r}$ in equation (41) may be obtained from the values of $b_{e}$ and $b_{r}$ referring to the constituents in the pure state. The value of $b^{\prime}{ }_{e}$ arises from the interaction of molecules $e$ with each other and with the molecules $r$, while the value of $b^{\prime}{ }_{r}$ arises from the interaction of the molecules $r$ with each other and with the molecules $e$. It is evident, therefore, that if $N_{r}$ denotes the concentration of the molecules $r$, and $v_{e}$ the volume of the mixture containing a gram molecule of molecules $e$, we have approximately

$$
b^{\prime}{ }_{e}=b_{e}+v_{e} \frac{N_{r}}{N}\left\{\frac{\sqrt[3]{b_{e}}+\sqrt[3]{b_{r}}}{2}\right\}^{3},
$$

where $N$ denotes the number of molecules in a gram molecule of a pure substance. The first term on the right-hand 
side of the equation expresses the apparent molecular volume of the molecules $e$ when a molecule $e$ interacts with them, while the second term expresses the apparent volume of the molecules $r$ when a molecule $e$ interacts with them. Similarly for $b^{\prime}{ }_{r}$ we have approximately

$$
b_{r}^{\prime}=b_{r}+v_{r} \frac{N_{e}}{N}\left\{\frac{\sqrt[3]{b_{e}}+\sqrt[3]{b_{r}}}{2}\right\}^{3},
$$

where $N_{e}$ denotes the number of molecules $e$ in a volume of the mixture containing a gram molecule of molecules $r$.

The expansion pressure $P_{e}$ may now be connected with other quantities.

21. The Intrinsic Pressure; and the Equation of Equilibrium of a Substance.

On account of the attraction between the moiecules of a liquid, or dense gas, a negative pressure is associated with it usually called the intrinsic pressure, which acts in

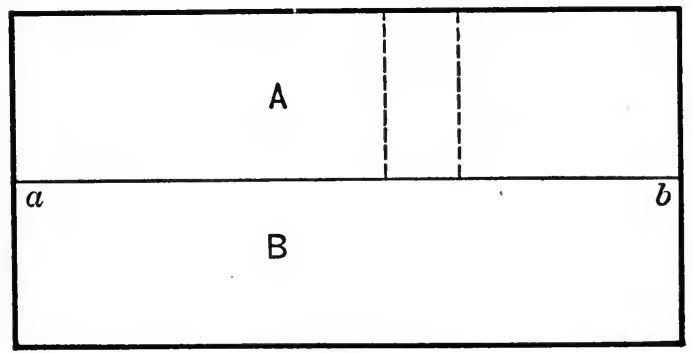

FIG. 7.

the opposite direction to the external pressure regarded from an external point. The existence of this pressure follows from the following consideration. Suppose a substance is cut into two parts $A$ and $B$ by an imaginary plane $a b$ as shown in Fig. 7. If attraction between the molecules exists the parts $A$ and $B$ exert an attraction 
upon each other which gives rise to a pressure between them. This pressure per square $\mathrm{cm}$. in the plane $a b$ is numerically equal to the intrinsic pressure, or negative pressure which the parts $A$ and $B$ exert upon each other.

The expansion pressure $P_{e}$ exerted by the molecules in crossing a square $\mathrm{cm}$. in the plane $a b$ is evidently balanced by the intrinsic pressure, which will be denoted by $P_{w}$, and the external pressure $p$, that is,

$$
P_{n}+p=P_{e}, \quad . \quad . \quad .
$$

or

$$
{ }^{\ulcorner} P_{n}+p=\frac{n v}{v-b} \frac{A}{2}=2.543 \times 10^{-20} \frac{n v}{v-b} \sqrt{T m}, .
$$

according to equation (38). The foregoing equation* is the equation of equilibrium of a substance. The quantity $P_{n}$ may be expressed very approximately in terms of quantities which may be measured directly, as will now be shown.

The average position of a molecule in a substance corresponds to a point about which the surrounding molecules are symmetrically situated. The molecular forces therefore neutralize each other at such a point. The average kinetic energy of a molecule at its average position is therefore the same as that it has in the perfectly gaseous state according to Section 16. Now we may suppose that each molecule occupies its average position in a substance at the same time for purposes of calculation, for the properties of a molecule in connection with its forming part of a substance are average properties. It appears then that if the volume of a substance is changed by $\delta v$ at constant temperature, the average minimum or temperature kinetic energy is not changed. Hence the heat $\delta U$ absorbed repre-

* Reconstructed form of an equation given by the writer in the Phil. Mag., July, 1912, pp. 101-108. 
sents, if no dissociation occurs - as we will suppose, the work $\delta U_{a}$ done against molecular attraction in separating the molecules, and in changing their internal molecular energy by $\delta u_{m}$. The change in energy $\delta u_{m}$ may be caused by a change in the atomic sonfiguration of the molecules, and a change in their velocities of rotation, etc. The latter energy is very likely small in comparison with the former, in which case we have

$$
P_{n} \cdot \delta v=\delta U_{a}=\delta U,
$$

since work is done against the intrinsic pressure during expansion. Now according to thermodynamics we have

$$
\left(\frac{\delta U}{\delta v}\right)_{T}=T\left(\frac{\delta p}{\delta T}\right)_{0}-p, \quad . \quad . .
$$

where $p$ and $T$ denote the pressure and absolute temperature of the substance, and hence

$$
P_{n}=T\left(\frac{\delta p}{\delta T}\right)_{0}-p=\frac{T \alpha}{\beta}-p, \ldots .
$$

since by the help of the Differential Calculus

$$
\left(\frac{\delta p}{\delta T}\right)_{0}=-\left(\frac{\delta v}{\delta T}\right)_{p} /\left(\frac{\delta v}{\delta p}\right)_{T}, \alpha=\frac{1}{v}\left(\frac{\delta v}{\delta T}\right)_{p}, \text { and } \beta=-\frac{1}{v}\left(\frac{\delta v}{\delta p}\right),
$$

where $\alpha$ denotes the coefficient of expansion at constant pressure, and $\beta$ the coefficient of compression at constant temperature of the substance. These coefficients can be measured directly and hence numerical values of $P_{n}$ be obtained. The values obtained in this way are likely to be very approximately correct, since the dissociation of the molecules with the expansion of a substance is usually negligible, and the change in internal molecular energy we would expect to be small in comparison with the work 
done against molecular attraction, i.e., against the intrinsic pressure.

Table III gives the values of the intrinsic pressure at different temperatures for a few liquids calculated* by

TABLE III

\begin{tabular}{|c|c|c|c|c|c|}
\hline \multicolumn{6}{|c|}{ Ether, $\left(\mathrm{C}_{4} \mathrm{H}_{10} \mathrm{O}\right)$. } \\
\hline$t^{\circ} \mathrm{C}$ & $\beta 10^{6}$ & $\alpha$ & $\rho$ & $\begin{array}{l}P_{n}=\frac{T \alpha}{\beta} \\
\text { atmos. }\end{array}$ & $p^{\prime}$ atmos. \\
\hline 13.5 & 169 & .001574 & .7214 & 2669 & 232.3 \\
\hline 25.4 & 190 & .001632 & .7077 & 2467 & 237.3 \\
\hline 63 & 300 & .001809 & .6620 & 2026 & 250.0 \\
\hline 78.5 & 367 & .001892 & .6421 & 1812 & 253.6 \\
\hline 99 & 539 & .001992 & .6105 & 1375 & 255.2 \\
\hline \multicolumn{6}{|c|}{ Benzene, $\left(\mathrm{C}_{6} \mathrm{H}_{6}\right)$. } \\
\hline 15.4 & 87 & .001215 & .8840 & 4083 & 271.8 \\
\hline 50.1 & 111 & .001305 & .8466 & 3796 & 291.6 \\
\hline 78.8 & 126 & .001379 & .8145 & 3850 & 305.5 \\
\hline \multicolumn{6}{|c|}{ Chloroform, $\left(\mathrm{CHCl}_{3}\right)$. } \\
\hline 0 & 101 & .001107 & 1.5264 & 2991 & 289.8 \\
\hline 20 & 128 & .001294 & 1.4885 & 2970 & 303.7 \\
\hline 40 & 162 & .001484 & 1.4503 & 2869 & 315.8 \\
\hline 60 & 204 & .001670 & 1.4108 & 2726 & 326.9 \\
\hline \multicolumn{6}{|c|}{ Pentane, $\left(\mathrm{C}_{5} \mathrm{H}_{12}\right)$. } \\
\hline 0 & 229 & .001465 & .6454 & 1747 & 203.5 \\
\hline 20 & 318 & .001589 & .6262 & 1337 & 211.9 \\
\hline 40 & 416 & .001721 & .6062 & 1294 & 219.2 \\
\hline 60 & 486 & .001830 & .5850 & 1260 & 225.0 \\
\hline
\end{tabular}

* R. D. Kleeman, Proc. Camb. Phil. Soc., Vol. XVI, Pt. 6 (1912), p. 545 . 
means of the foregoing formula, and for comparison the values of the external pressures $p^{\prime}$ given by $p^{\prime}=\frac{R T \rho}{m}$ which the substances would have if they behaved as perfect gases.

The values of the coefficient $\beta$ in the Table refer to the compression per atmosphere, and hence the values of $P_{n}$ are expressed in terms of the same quantity. The values of $p^{\prime}$ have therefore also been expressed in terms of this quantity by dividing the value of $R$ in the gas equation (Section 5) by $10^{6}$. The values of $\alpha, \beta$, and $\rho$, were taken from Landolt and Börnstein's Tables, the values of $p$ being unnecessary, since they are so small in comparison with those of $T_{\bar{\beta}}^{\alpha}$ that they may be neglected. The values of $P_{n}$ are striking on account of their magnitude, being much greater than $p^{\prime}$, and illustrate the powerful attraction that exists between molecules for close distances of approach. Since the attraction of a molecule probably increases with its mass, the magnitude of the intrinsic pressure for constant volume probably varies in a similar way. It would evidently decrease with the density $\rho$ of the substance since the attraction between two molecules decreases with their distance of separation.

The intrinsic pressure is evidently greater than the external pressure, and this holds therefore also for the expansion pressure $P_{e}$ according to equation (45), or $P_{n}>p$ and $P_{e}>p$. Also according to this equation $P_{e}>P_{n}$, and since $P_{n}>p^{\prime}$ according to the Table, we also have $P_{e}>p^{\prime}$. The result that the expansion pressure $P_{e}$ of a liquid is greater than the external pressure $p^{\prime}$ it would have if it behaved as a perfect gas is caused according to Section 20 by the molecules possessing an apparent volume, and that the total average velocity of a molecule is greater than the velocity it would have in the perfectly gaseous state, or that $b>0$ and $V_{\imath}>V_{a}$. 
If the equation of state (Section 26) of a substance be known the values of $\alpha$ and $\beta$ may be calculated for different temperatures and densities of a substance by means of the equations defining these quantities, or better, the value of $\left(\frac{\delta p}{\delta T}\right)_{0}$ may be directly calculated and hence the corresponding intrinsic pressure be obtained.

It is possible now to obtain superior and inferior limits of the values of $n$ and $V_{t}$ of a substance.

\section{Superior and Inferior Limits of $n$ of $a$} Substance.

It will appear from an inspection of equation (46) that a superior limit of $n$, the number of molecules crossing a square $\mathrm{cm}$. from one side to the other per second, which will be written $n^{\prime \prime}$, is obtained by supposing that the apparent volume of the molecules is zero, or $b=0$, in which case the equation becomes

$$
n^{\prime \prime}=\frac{P_{n}+p}{2.543 \times 10^{-20}} \sqrt{ } \overline{T m} .
$$

The quantity $P_{n}$ on the right hand side of this equation, we have seen, can be calculated from quantities determined by experiment.

If $P_{n}$ is zero, $V_{t}$ has the same value as if the substance were in the perfectly gaseous state, since it is independent of the apparent molecular volume (Section 19). Hence according to equations (35) and (8), the value of $n$ under these conditions, which will be written $n^{\prime}$, is given by

$$
n^{\prime}=\frac{N_{c} V_{t}}{3}=\frac{N_{c}}{3} \sqrt{\frac{3 R T}{m}} .
$$

This is an inferior limit of $n$ since $V_{t}$ cannot have a smaller value than corresponding to the perfectly gaseous state. 
Table IV contains as an illustration the superior and inferior limits of $n$ for a few substances in the liquid state*. The values of $P_{n}$ used in the calculations are given in the Table III.

TABLE IV

\begin{tabular}{|c|c|c|c|c|c|}
\hline \multicolumn{3}{|c|}{ ETHER } & \multicolumn{3}{|c|}{ Pentane } \\
\hline$t^{\circ} \mathrm{C}$ & $\operatorname{Sup}_{\text {of } n} \lim _{10-25}$. & $\begin{array}{l}\text { Inf. } \operatorname{Lim}_{\text {of }} \text {. } \\
\text { 10-25. }\end{array}$ & $t^{\circ} \mathrm{C}$ & $\begin{array}{l}\text { Sup. } \operatorname{Lim} . \\
\text { of } n \text { 10-25. }\end{array}$ & $\begin{array}{l}\text { Inf. Lim. } \\
\text { of } n 10^{-25} \text {. }\end{array}$ \\
\hline 13.5 & 71 & 6.24 & 0 & 49 & 5.23 \\
\hline 78.5 & 44 & 6.16 & 60 & 32 & 5.68 . \\
\hline \multicolumn{3}{|c|}{ Chloroform } & \multicolumn{3}{|c|}{ Benzene } \\
\hline 0 & 65 & 6.30 & 15.4 & 108 & 7.0 \\
\hline 60 & 54 & 6.50 & 78.8 & 94 & 7.12 \\
\hline
\end{tabular}

It will be seen that in the case of each substance one of the limits is many times that of the other, the actual value of $n$ of course lying between the two. Since the values of $P_{n}$ are large in comparison with the values of $p^{\prime}$ (the external pressure a substance would have in the perfectly gaseous state (Section 21)), and the former are the outcome of molecular attraction which in proportion effects the velocities of translation of the molecules, and therefore the values of $n$, we would expect the actual values of $n$ to lie nearer to the superior than to the inferior limits obtained.

23. Superior and Inferior Limits of $V_{t}$ of $a$ Substance.

These limits are obtained by substituting in equation (35) the superior and inferior limits of $n$ obtained by the

${ }^{*}$ If the values of $n$ are corrected according to Maxwell's law they have to be divided by 1.085 according to Section 20 . 
method given in the previous Section. Table V gives the values obtained in this way for a few substances. For reasons stated in the previous Section we would expect that the actual total average velocity of a molecule in a substance should lie nearer 'o the superior, than to the inferior limit. The inferior limit is the velocity when the substance is in the perfectly gaseous state.

\section{TABLE V}

\begin{tabular}{|c|c|c|c|c|c|}
\hline \multicolumn{3}{|c|}{ Ether } & \multicolumn{3}{|c|}{ Pentane } \\
\hline$t^{\circ} \mathrm{C}$. & $\left\{\begin{array}{c}\text { Sup. Lim. } \\
\text { of } V_{t} 10^{+} \frac{\mathrm{cms}}{\mathrm{gec}}\end{array}\right.$ & $\begin{array}{l}\text { Inf. Lim. } \\
\text { of } V_{l} 10^{4} \frac{\mathrm{cms}}{\mathrm{sec} .}\end{array}$ & $t^{\circ} \mathrm{C}$ & $\begin{array}{l}\text { Sup. Lim. } \\
\text { of } V_{t} 10^{4} \frac{\mathrm{cms}}{\mathrm{sec}}\end{array}$ & $\begin{array}{l}\text { Inf. Lim. } \\
\text { of } V_{t} 10^{4} \frac{\mathrm{ems}}{\mathrm{sec} .}\end{array}$ \\
\hline 13.5 & 35.7 & 3.10 & 0 & 26.4 & 2.83 \\
\hline \multicolumn{3}{|c|}{ ChLoroform } & \multicolumn{3}{|c|}{ Benzene } \\
\hline 0 & 24.6 & 2.38 & 15.4 & 46.0 & 2.98 \\
\hline
\end{tabular}

The quantities $V_{t}$ and $n$ do not depend on the quantity $b$ by definition according to Section 19 . Therefore if $P_{n}$ and $p$ were also independent of $b$, equation (49) would give the actual value of $n$ instead of a superior limit, while equation (35) would give the actual value of $V_{t}$. But $p$ obviously depends on $b$ (Section 19); it is, however, small in comparison with $P_{n}$ in the cases considered. Therefore if $b$ did not depend on the molecular forces $P_{n}$ would be independent of $b$, and the superior limits of $n$ and $V_{\imath}$ obtained would very approximately represent the actual values of these quantities. There is some indirect evidence, however, with which we will get acquainted as we proceed, that $b$ is the outcome of molecular forces, as we would expect.

The mathematical definition of $b$ was given in Section 19 , but so far no information about its value and properties 
has been discussed. It will be convenient now to discuss the quantity from a certain aspect.

\section{The Real and Apparent Volumes of a} Molecule, and their Superior Limits.

The property of volume of a substance is known to us only through the resistance to force: thus if two portions of matter are pressed together there is a resistance to the operation which need not be accounted for by actual contact taking place-whatever that may mean, but by an approach of the molecules of the substances to such distances that the resultant of their forces of repulsion equals the force with which the substances are pressed together. It seems unsafe therefore, and little warranted by the facts, to associate with an atom an impenetrable perfectly elastic volume of constant magnitude. There is no doubt, though, that it may be said that a volume is associated with each molecule through which the center of another molecule cannot pass, but whose magnitude depends upon external conditions. If this volume is due to the existence of forces of repulsion, as is highly probable, its magnitude will depend upon the force exerted in approaching another molecule. Thus it is evident that in the case of a gas this volume will decrease with increase of temperature, for this is attended by an increase in the kinetic energy of the molecules, and therefore by a decrease in the minimum distance of approach, the molecules approaching each other to a distance at which their kinetic energy is completely or partly converted into potential energy of repulsion. It seems futile, therefore, to expect to obtain a constant value for the quantity in question since it varies with the external conditions, which often are not altogether known. The best we can hope to obtain is the order of magnitude of the quantity, which is likely to be always the same. 
The apparent volume of a molecule, or of a gram molecule of molecules, which was investigated in Section 19, is, however, a perfectly definite mathematical quantity. But its connection with the "real volume," whatever that may mean, cannot be ascertained with certainty, if at all, since the apparent volume would depend on the geometrical configuration of the "real volume." Thus for example, Van der Waals has shown that if the "real volume" of a molecule consists of an impenetrable sphere, the apparent volume is four times that of the "real volume." Now if the apparent volume is caused by forces of repulsion between the molecules, it is difficult, if not impossible, to define exactly what is meant by the "real volume," and therefore to determine its magnitude. It will be evident on reflection that the "real volume" of a molecule is not exactly the volume through which another molecule will never pass.

The volume occupied by a molecule, or associated with a molecule, at absolute zero, is of special interest in this connection. Since at that temperature the molecules have no motion of translation each will take up a position so that the forces of attraction and repulsion acting upon it balance each other, in other words, since $P_{e}$ and $p$ are zero $P_{n}$ is zero according to equation (45). The apparent and "real volume" are evidently equal to each other under these conditions. The apparent volume of a molecule at the absolute zero is likely to be greater than at a higher temperature, since the velocity of the molecules due to their temperature is likely to make the molecules approach closer to each other in opposition to their repulsion (Section 15) than they would otherwise. This volume is thus a superior limit of the apparent molecular volume for higher temperatures than the absolute zero. An approximate value of this volume at the absolute zero may be obtained from considerations involving the coefficient of expansion, or by means of Cailletet and Mathias' linear diameter 
law. According to this law the densities $\rho_{1}$ and $\rho_{2}$ of a liquid and its saturated vapor respectively are given by the equation

$$
\rho_{1}+\rho_{2}=a+c T,
$$

where $T$ denotes the absolute temperature, and $a$ and $c$ denote constants depending only on the nature of the liquid. These constants may be determined from values of $\rho_{1}$ and $\rho_{2}$ corresponding to two different temperatures of the liquid. At the absolute zero, or $T=0$, we accordingly have $\rho_{1}=a$, since $\rho_{2}=0$ and $T=0$. As an illustration the values of $v_{0}$ of a gram molecule for a number of substances at the absolute zero, or the values of $m / \rho_{1}$, and the corresponding values of $v_{0}^{\prime}$ and $\left(v^{\prime}\right)^{1 / 3}$ for a single molecule, are given in Table VI. It will be seen that the values of

TABLE VI

\begin{tabular}{|c|c|c|c|c|c|}
\hline Substance. & $\begin{array}{r}v_{0} . \\
\text { c.c. }\end{array}$ & $\begin{array}{l}{ }^{v}{ }^{v} \\
\text { c.c. }\end{array}$ & $\frac{v_{c} c}{v_{0}}$. & $\begin{array}{c}v_{0}^{\prime} 10^{24} . \\
\text { c.c. }\end{array}$ & $\begin{array}{l}\left(v_{0}\right)^{1 / 3} \\
10^{8} . \\
\mathrm{cm} .\end{array}$ \\
\hline Oxygen... & 20.8 & 49.2 & 2.37 & 33.5 & 3.22 \\
\hline Nitrogen..... & 25.0 & 70 & 2.80 & 40.2 & 3.42 \\
\hline Carbon dioxide. & 25.5 & 96 & 3.77 & 41.1 & 3.44 \\
\hline Ether........ & 71.7 & 280 & 3.91 & 115 & 4.87 \\
\hline Benzene..... & 70.6 & 256 & 3.63 & 114 & 4.84 \\
\hline Carbon tetrachloride. & 72.2 & 276 & 3.82 & 116 & 4.88 \\
\hline Propyl acetate...... & 86.2 & 345 & 4.00 & 139 & 5.18 \\
\hline
\end{tabular}

$v_{0}^{\prime}$ increase with increase of molecular weight, as we would expect. The values of $\left(v^{\prime}\right)^{1 / 3}$ probably give the order of magnitude of the apparent diameter of a molecule under various conditions. The Table also gives the values of the critical volume $v_{c}$, and the values of the ratio $v_{c} / v_{0}$, which, it will be seen, lie in the neighborhood of 4 . The values of $v_{0}$ and $v_{c}$ were taken from a Table given in Nernst's Theoretische Chemie, 7th ed., p. 234. A superior limit of $b$ is thus $v_{c} / \mathbf{4}$. 
An important application of the foregoing results may immediately be made.

\section{Inferior Limits of $n$ and $V_{t}$.}

The results of the preceding Section enable us to obtain another inferior limit for $n$ of a substance by means of equation (46), on writing $b=v_{c} / 4$, which is a superior limit of $b$. On substituting the inferior limit of $n$ thus obtained in equation (35) it gives an inferior limit of $V_{t}$. Table VII

TABLE VII

\begin{tabular}{|c|c|c|c|c|c|}
\hline & \multicolumn{2}{|c|}{$\mathrm{CO}_{2}$ at $40^{\circ} \mathrm{C}$} & \multicolumn{2}{|c|}{$V_{a}=4.165 \times 10^{4} \frac{\mathrm{cms}}{\mathrm{sec} .}$} & \multirow[b]{2}{*}{$\begin{array}{l}\text { Inf. Lim. } \\
\text { of } V_{t} 10^{4} \frac{\mathrm{cms}}{\mathrm{sec}}\end{array}$} \\
\hline $\begin{array}{c}p \text { in } \\
\text { atmos. }\end{array}$ & $\begin{array}{l}\text { Inf. Lim. } \\
\text { of } n 10^{2} \text {. }\end{array}$ & $\begin{array}{l}\text { Inf. Lim. } \\
\text { of } V_{t} 10^{4} \frac{\mathrm{cms}}{\text { sec. }}\end{array}$ & $\begin{array}{l}p \text { in } \\
\text { atmos. }\end{array}$ & $\begin{array}{l}\text { Inf. Lim. } \\
\text { of } n 10^{20} \text {. }\end{array}$ & \\
\hline 70 & 3.88 & 4.60 & 94 & 14.4 & 5.94 \\
\hline 80 & 5.76 & 4.79 & 100 & 16.4 & 6.24 \\
\hline 85 & 8.01 & 5.06 & 112 & 18.7 & 6.63 \\
\hline
\end{tabular}

contains the inferior limits for $\mathrm{CO}_{2}$ at $40^{\circ} \mathrm{C}$. calculated from the values of $P_{n}+p$ obtained in Section 34 and given in Table XIV. It will be seen that at constant temperature the inferior limit of $V_{t}$, the total average molecular velocity, gradually increases with increase of pressure or density of the substance, and that its value corresponding to an external pressure of 112 atmos. is about 50 per cent greater than the values of $V_{a}$, the total average velocity of a molecule of the substance in the perfectly gaseous state at the same temperature*. This definitely shows that the total average molec-

* The values obtained for $\mathrm{V}_{t}$ and $\mathrm{V}_{a}$ have not been corrected for an uneven distribution of molecular velocities. According to Maxwell's Law of Distribution each value has to be divided by 1.085 according to Sections 8, 11, and 20. 
ular velocity at constant temperature in a substance not obeying the laws of a perfect gas increases with the density, and that it may be considerably larger than the velocity when the substance is in the perfectly gaseous state. The results thus fall into line with those of Sections 16 and 17, and we will see later with those of Section 29 .

From this it follows that $n$ according to equation (35) increases more rapidly with the density of a substance than proportionally to it.

In the previous Sections we have mainly considered individual properties of molecules. It will be of interest and importance now to consider some properties of a substance as a whole which depend on the dynamical and attractional properties of the molecules.

\section{The Equation of State of a Substance.}

The state of a given mass of a substance according to experiment is completely defined by two variables. Therefore each of the variables of a substance may be expressed in terms of any two by means of an equation. Such a relation is called an equation of state of the substance, and contains two independent variables. The most useful equation is that connecting the variables pressure, volume, and temperature.

One of the equations of state is

$$
p+P_{n}=P_{e}, \quad \cdot \quad \cdot \quad \cdot \quad \cdot
$$

obtained in Section 21, where $p, P_{n}$, and $P_{e}$ denote the external, intrinsic, and expansion pressures respectfully of the substance. This equation may also be written

$$
p+P_{n}=2.534 \times 10^{-20} \frac{n v}{v-b} \sqrt{T m}, . .
$$

according to equation (38), where $n$ denotes the number of molecules crossing a square $\mathrm{cm}$. per second, and $b$ the appar- 
ent volume of the molecules. According to equation (40) and subsequent remarks the preceding equation may also be written

$$
P_{n}+p=\frac{V_{t}}{V_{a}} \frac{R T}{v-b}, \quad \cdots \quad .
$$

where $V_{t}$ denotes the total average velocity of a molecule of a substance for any state, $V_{a}$ the average velocity in the gaseous state, $v$ the volume of a gram molecule, $b$ the apparent molecular volume, and $R$ the gas constant. The foregoing equations are fundamental in character, since they do not depend upon any hypothesis.

If equation (52), (like (53)), is corrected according to Maxwell's law of distribution of molecular velocities in the gaseous state, the right-hand side of the equation has to be multiplied by $\sqrt{\frac{3 \pi}{8}}$, or 1.085 , according to Section 20 .

The problem that remains to be solved in connection with the foregoing equations is to find an expression for the quantities, $P_{e}, n, V_{t}$, and $b$, in terms of other quantities, particularly in terms of the quantities $p, v$, and $T$, which can be measured directly. This cannot be done without the introduction of assumptions, and therefore yields results which are likely to hold only approximately.

Van der Waals has proposed the equation of state

$$
p+P_{n}=p+\frac{a}{v^{2}}=\frac{R T}{v-b}, . . . .
$$

where $a$ and $b$ are constants. On comparing this equation with equation (53) we see that $P_{n}=a / v^{2}$, and $V_{t}=V_{a}$. According to the latter equation the velocity of translation of the molecules is not influenced by molecular attraction. But this is inadmissible according to Sections 17, 20, and 25 , nor does it hold approximately, for we will see in Section 
29 that when a substance is in the liquid state the value of $V_{t}$ may be more than four times the value of $V_{a}$. Thus the values of $b$ calculated from Van der Waals' equation would be too large.

Van der Waals deduced his equation from the suppositions that the total average velocity of a molecule is not affected by molecular attraction, or is the same as the velocity in the gaseous state, and $b$ represents the apparent volume of a gram molecule of molecules as a whole due to a true impenetrable spherical volume being associated with each molecule. The value of $b$ is then four times the true volume occupied by the molecules.

The equation from Van der Waals as a whole, however, fairly well represents the facts, that is, the values of each of the quantities $p, v$, and $T$, may be obtained by means of it given the corresponding values of the remaining two quantities. The equation is particularly useful for such determinations on account of its simplicity.

It can easily be shown that the intrinsic pressure term $a / v^{2}$ in Van der Waals' equation corresponds to attraction between two molecules varying inversely as the fourth power of their distance of separation. Thus the molecules of a substance may be divided into a number of parallel rows separated from each other by a distance $x_{1}$, the distance of separation of the molecules. Therefore if $A_{1} / x^{s}$ represents the law of molecular attraction, the attraction of onehalf of a row on the other half may evidently be written $A_{2} / x^{s}$, where $A_{2}$ represents an appropriate constant. Since $1 / x^{2}$ rows pass through a square $\mathrm{cm}$. situated at right angles to the rows, the attraction of the molecules in one-half of a oylinder of unit cross-section and infinite length on the molecules in the other half is given by

$$
\frac{A_{2}}{x^{s+2}}
$$


and this is the intrinsic pressure. Now $x_{1}=\left(\frac{m_{a}}{\rho}\right)^{1 / 3}=\left(\frac{v m_{a}}{m}\right)^{1 / 3}$, where $\rho$ denotes the density, and $v$ the volume of a gram molecule of the substance, and thus the intrinsic pressure may be written

$$
A_{3} \rho^{\frac{s+2}{3}}, \text { or } \frac{A_{4}}{v^{\frac{8+2}{3}}}
$$

where $A_{3}$ and $A_{4}$ are constants. Therefore when $\frac{s+2}{3}=2$, $s=4$.

The effect of molecular attraction is, however, not so simple that it may be expressed by a single term. According to Section 14 it should contain at least two additional terms having negative signs which express repulsion between the molecules. The attraction terms are probably approximately represented by the foregoing single term involving the inverse fourth power. The subject of molecular attraction and its bearing on the form of the intrinsic pressure term is not of primary importance in connection with the object of this book, and will therefore not be discussed here any further but reserved for another place. For purposes of calculating the values of one of the variables $p, v$, and $T$, from given values of the other two variables most of the empirical equations of state answer equally well. An account of a number of them will be found in Winckelmann's Handbuch der Physik, II. Edition, pp. 1135-1143.

Equation (53) may be given a form by means of which the values of $V_{\imath}$ and $P_{n}$ of a substance may approximately be calculated. The quantity $V_{t}$ may be taken equal to the sum of two terms, one equal to $V_{a}$, and the other equal to $V_{1}$, which represents the effect of molecular attraction on the value of $V_{t}$. Now a molecule will pass over a distance proportional to $x_{1}$ in passing out of the influence of one mole- 
cule and under the influence of another. Therefore if $A_{1} / x^{8}$ represents the law of molecular attraction the change of kinetic energy of the molecule over that distance may be written $\frac{D_{1} x_{1}}{x_{1}^{s}}$, and the velocity $V_{1}$ is therefore approximately given by

$$
\frac{D_{2}}{x_{1}^{\frac{s-1}{2}}}, \text { or } \frac{D_{3}}{v^{\frac{s-1}{6}}} \text {, }
$$

where $D_{3}$ is a constant. Equation (53) may accordingly be written

$$
p+\frac{A_{4}}{v^{\frac{s+2}{3}}}=\left(V_{a}+\frac{D_{3}}{v^{\frac{s-1}{6}}}\right) \frac{R T}{V_{a}(v-b)}
$$

where $V_{a}=\sqrt{\frac{8}{3 \pi}} \sqrt{\frac{3 R T}{m}}$ according to Sections 6 and 8 .

On applying this equation to three states of a substance not differing much from one another at constant temperature the quantities $A_{4}, D_{3}$, and $b$, may be taken as constant and determined from these equations. The values of $V_{t}$ and $P_{n}$ corresponding to these three states may then at once be calculated. Similarly the equation may be applied to another set of three states and the corresponding values of $V_{t}, P_{n}$ and $b$, obtained, and so on. The values of $V_{t}$ and $b$ obtained in this way by means of equation (55) will obviously not be so accurate as those obtained by means of equation (67) in Section 29. The former equation can, however, be more readily applied to the facts. The values of $b$ obtained by this equation should more accurately represent the facts according to the definition of $b$ in Section 19 than those obtained by Van der Waals' equation, since $V_{t}$ is not taken equal to $V_{a}$. If $s$ is not known it may be determined by applying equation (55) to four different states of the substance. 
The quantities $P_{e}$ and $P_{n}$ in the equation of state must very approximately satisfy the relation obtained on eliminating $p$ from equations (51) and (48), which gives

$$
P_{e}=T\left\{\left(\frac{\delta P_{e}}{\delta T}\right)_{0}-\left(\frac{\delta P_{n}}{\delta T}\right)_{0}\right\}
$$

It follows from this equation that if $P_{n}$ is a function of $v$ only $P_{e}$ is of the form $T \cdot \phi(v)$, where $\phi(v)$ is a function of $v$. Van der Waals' equation of state satisfies the above condition, but of course it does not follow that therefore its form is correct. It is fairly certain that $P_{n}$ is a function of $T$ as well as of $v$. For the intrinsic pressure of a substance, since it depends upon molecular attraction, will depend upon the change in atomic configuration of a molecule with change in temperature. Since such a change undoubtedly occurs, as is shown by the deviation of the ratio of the specific heats considered in Section 13 from the value 1.66, the form of the function for $P_{e}$ is not as simple as a linear function of $T$.

\section{The Conditions that the Equation of State} has to satisfy.

If the pressure of a substance is plotted against its volume at different temperatures, curves of the form shown in Fig. 8 are obtained. For certain temperatures each curve consists of two parts, each of which has one of the terminal points lying on a line (dotted in the figure) parallel to the volume axis. Thus corresponding to the pressure indicated by the dotted line the substance can only have either of the two volumes corresponding to the abscissæ of the points at the extremities of the line. As the temperature of the substance is increased the length of the dotted line is decreased, and for a certain temperature, called the critical temperature, it entirely disappears. 
Van der Waals has proposed a theory to explain this behavior of substances. According to it the two curves corresponding to a given temperature are theoretically parts of a single curve as shown in Fig. 9, the form of the absent part being such as to render the curve continuous. The fact that the intermediate part of the curve is not realized in practice is explained by supposing that a sub-

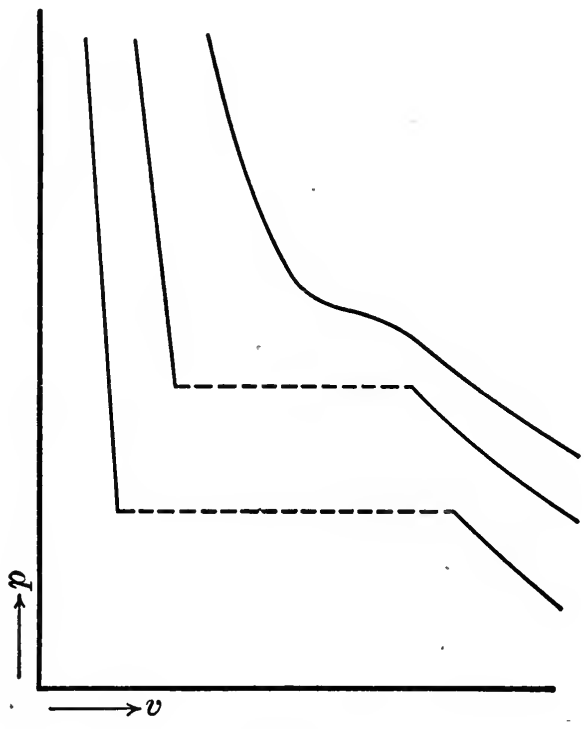

FIG. 8.

stance in each of the states represented by it is in unstable equilibrium, accordingly a disturbance from the outside would make the substance change to one or both the stable forms represented by the points $a$ and $b$ at the extremities of this part of the curve.

Thus according to this theory there is no discontinuity of state; and therefore the equation connecting $p$ and $v$ 
for the two stable portions of a curve will also represent the unstable condition.

It will appear from an inspection of the curves in Fig. 9 that the point $e$, which corresponds to the critical point,

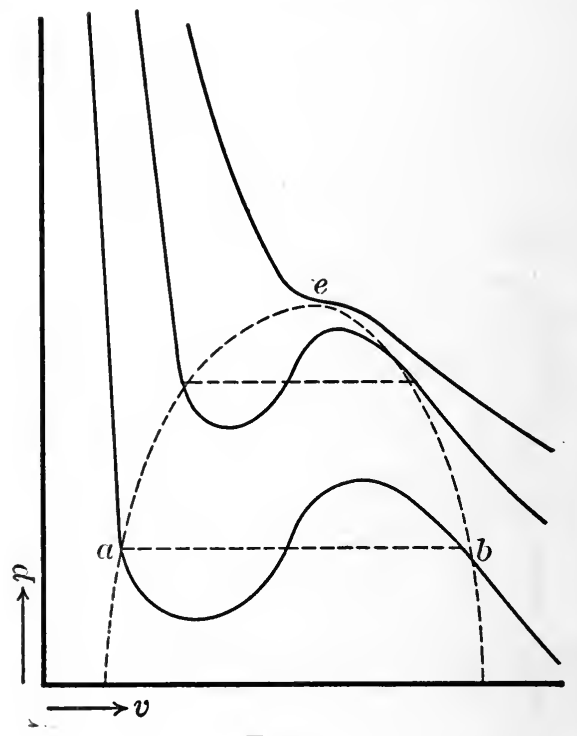

Fia. 9.

is a point of inflexion. The geometrical properties of the point are expressed by the equations

$$
\left(\frac{\delta p}{\delta v}\right)_{T}=0, \quad . \quad . \quad . \quad . \quad .
$$

and

$$
\left(\frac{\delta^{2} p}{\delta v^{2}}\right)_{T}=0, \quad \cdot \quad \cdot \quad \cdot \quad \cdot
$$

which the equation of state has to satisfy at the critical point. These equations express relations between the constants of the equations of state and the critical constants. 
The foregoing equations, it may be mentioned, can also be deduced from the Laws of Thermodynamics.

The equation of state must also possess the property that it vanish if the substitutions $p=0, T=0$, and $v=\infty$, are made, which correspond to the absolute zero of temperature. This may indicate that a relation exists between the coefficients of the equation, or that it has a certain form.

If the temperature and volume at constant pressure of a substance are plotted against each other instead of the pressure and volume at constant temperature, a set of curves similar to those shown in Fig. 8 is obtained. A portion of each curve cannot be realized in practice. The theoretical curve representing this portion must for reasons of continuity cut a volume ordinate in three points, similarly as shown in Fig. 9. But this cannot hold at the absolute zero, since the absolute temperature cannot have negative values, and therefore no part of the curve can lie on the negative side of the volume axis. The part of the curve lying between the volumes $v_{0}$ and infinity inclusive in that case coincides with the volume axis. This condition is expressed by the equation

$$
\left(\frac{\delta T}{\delta v}\right)_{p}=0, \quad \cdot \quad \cdot \quad \cdot \quad \cdot .
$$

which holds for $v=$ infinity, $v=v_{o}$, and inclusive values, corresponaiing to $p=0$, and $T=0$. This probably indicates that the equation of state must have a certain general form which satisfies this condition.

Experiment shows that the variables pressure, volume, and temperature, of a liquid in contact with its saturated vapor, may each be expressed by an equation in terms of one of the variables, and this holds also for the saturated vapor. It can be shown that this is a consequence of Van der Waals' theory of the continuity of state, and the Laws of Thermodynamics. Thus it follows from thermodynamics 
that the work done during an isothermal process between. given limits is independent of the path of the process. Hence the work done in passing from the point $a$ to the point $b$ in Fig. 9 is the same for the two paths indicated, one of which corresponds to that obtained in practice with a substance, while the other to that given by the equation of state. This condition is expressed by the equation

$$
p\left(v_{2}-v_{1}\right)=\int_{v_{1}}^{v_{2}} p \cdot \delta v, \quad . \quad . \quad .
$$

where the suffixes 1 and 2 refer to the liquid and vaporous states respectively.

On eliminating two of the variables $p_{1}, v_{1}, v_{2}$, and $T$ from the foregoing equation and the two equations obtained by applying the equation of state to the liquid and the vaporous state of the substance, a number of equations are obtained each of which contains two variables only.

Another set of equations each of which contains two variables only may be obtained as follows. * According to Clapeyron's equation the internal heat of evaporation $L$ of a gram molecule of a substance is given by

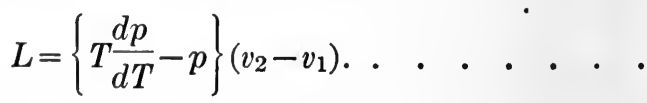

Now according to equation (47)

$$
L=\int_{v_{1}}^{v_{2}} U \cdot \delta v=\int_{v_{1}}^{v_{2}}\left\{T\left(\frac{\delta p}{\delta T}\right)_{0}-p\right\} \delta v,
$$

and therefore Clapeyron's equation by the help of equation (60) may be written

$$
\int_{v_{1}}^{r_{2}}\left(\frac{\delta p}{\delta T}\right)_{0} \cdot \delta v=\frac{d p}{d T}\left(v_{2}-v_{1}\right) . . . . .
$$

* R. D. Kleeman, Phil. Mag., Sept., 1912, pp. 391-102. 
From this equation and the equation of state, a number of equations may be obtained similarly, as before, each of which contains two variables only.

Now if one of the variables is eliminated from two equations taken from the foregoing two sets of equations which contain the same two variables, an equation is obtained containing one variable only. This equation must evidently identically vanish with respect to this variable. This requires that some of the constants in the equation be equated to zero. Since these constants are functions of the constants of the equation of state, the equations thus obtained express relations between the latter constants. The number of these equations evidently depends on the form assumed for the equation of state.

It appears, therefore, that the equation of state has to satisfy a number of conditions which govern its form and the relation between its constants. Other equations of condition besides those pointed out may of course exist, which have not yet been discovered.

The equation of state possesses besides an important property which indicates certain properties of its constants. This property, which is discussed in the next Section, is based upon the facts, but a theoretical reason may also be found for it.

\section{The Relation of Corresponding States.}

The quantities $p, v$, and $T$, of a substance may be expressed as fractions or as multiples $r_{1}, r_{2}$, and $r_{3}$, of their critical values thus: $p=r_{1} p_{c}, v=r_{2} v_{c}$, and $T=r_{3} T_{c}$. According to the relation of corresponding states if the value of each of two of the quantities $r_{1}, r_{2}$, and $r_{3}$, for each of a number of substances be taken the same, the remaining quantity has the same value for each substance. In the case that 
each substance consists of two phases in equilibrium, and one of the foregoing quantities is taken the same for each substance, each of the remaining two quantities is the same for each substance. Substances under these conditions are said to be at corresponding states, or obey the relation of corresponding states. This relation was first deduced by Van der Waals from his equation of state by applying to it the conditions of the critical point expressed by equations (57) and (58). The resultant equations (one of which is the equation of state), on eliminating the constants they contain, give an equation of the form

$$
\phi\left(\frac{p}{p_{c}}, \frac{v}{v_{c}}, \frac{T}{T_{c}}\right)=0,
$$

which expresses the first part of the relation in question. The second part follows from the fact that the equations of state of each of the phases of the two phases in equilibrium can be obtained from the foregoing equation and the thermodynamical equation (60), giving three equations each of which involves only two of the above ratios.

Meslin* has investigated the general conditions under which the relation may be deduced. The equation of state in its most general form is

$$
\psi_{1}\left(p, v, T, g_{1}, g_{2}, \ldots\right)=0
$$

where $g_{1}, g_{2} \ldots$, are constants depending only on the nature of the substance. Suppose that the various equations of condition applying to the equation of state are equal in number to the foregoing constants. On eliminating these constants an equation is obtained which is of the form

$$
\begin{gathered}
\psi_{2}\left(p, v, T, p_{c}, v_{c}, T_{c}\right)=0 . \\
{ }^{*} \text { Comp. Rend., 116, 135, } 1893
\end{gathered}
$$


This equation must be independent of the nature of the units chosen and therefore be of the form

$$
\psi_{2}\left(\frac{p}{p_{c}}, \frac{v}{v_{c}}, \frac{T}{T_{c}}\right)=0 \text {. }
$$

Therefore if $\psi_{2}$, (or $\psi_{1}$ ), has the same fundamental form for each substance the relation of corresponding states at once follows.

The number of equations of condition applying to a substance is not exactly known according to the previous Section, but it is evidently greater than two, and the number of constants that must be associated with an equation of state to obtain the relation of corresponding states is therefore at present undetermined. If we are given the relation of corresponding states, it obviously follows that the number of the foregoing equations of condition is equal to the number of constants in the equation of state.

The relation has been tested by a number of observers of whom Young may be especially mentioned, and found to agree fairly well with the facts. (An account of these investigations will be found in Winckelmann's Handbuch der Physik, 2d Edition, pp. 936-946). The deviations that occur are probably entirely due to polymerization, which renders a substance a mixture, and to which the relationship is not likely to apply.

The relation may be extended to a number of other quantities besides those mentioned which occur frequently in this book. According to Section 21

$$
P_{n}=\frac{T \alpha}{\beta}=T \frac{\delta v}{\delta T} / \frac{\delta v}{\delta p}-p .
$$

On substituting for $p, v$, and $T$ from the equations expressing these quantities in terms of their critical values we obtain

$$
P_{n}=\left\{r_{3} \frac{\delta r_{2}}{\delta r_{3}} / \frac{\delta r_{2}}{\delta r_{1}}-r_{1}\right\} p_{c}=r_{4} p_{c}, \quad . \quad . .
$$


where $r_{4}$ is a corresponding state quantity. At the critical point the equation becomes

$$
P_{n c}=r^{\prime}{ }_{4} p_{c}
$$

where $r^{\prime}{ }_{4}$ is a constant, and thus the preceding equation may be written

$$
P_{n}=r_{5} P_{n c}, \quad . \quad . \quad . \quad \text {. }
$$

where $r_{5}$ is a factor obeying the relation of corresponding states, and $P_{n c}$ denotes the intrinsic pressure at the critical point. Equation (63) can immediately be tested by the facts. The intrinsic pressures of ether and benzene in the liquid states at temperatures oorresponding to $2 T_{c} / 3$ can be calculated by the method of Section 21 and are found to be 2756 , and 3893 atmos. respectively. The ratio of these pressures is .708 , while the corresponding ratio of the critical pressures is .733 , which is practically equal to the preceding ratio, as should be the case.

It can be shown in a similar way that the quantities $n^{\prime \prime}$ and $V^{\prime \prime}{ }_{t}$, which are superior limits of the quantities $n$ and $V_{t}$, Sections (22) and (23), should also obey the relation of corresponding states.

If the quantity $b$ in the equation (52) may be expressed as a fraction obeying the relation of corresponding states of the critical volume $v_{c}$, or of the volume at the absolute zero, which is highly probable, it can be deduced in a similar way from this equation and equations (63) and (35) that $n$ and $V_{t}$ obey the relation of corresponding states. But even if $b$ did not possess this property, $V_{l}$ would obey the foregoing relation approximately since $b$ is usually small in comparison with $v$.

It will be of importance now to discuss a method of obtaining the actual values of the foregoing quantities in any given case. 
29. The Determination of the Quantities $n$, $V_{\imath}$, and $b$, of a Substance.

These quantities may most conveniently be determined by means of the equation

$$
\frac{T \alpha}{\beta}=\frac{A}{2} \frac{n v}{v-b}=2.543 \times 10^{-20} \frac{n v}{v-b} \vee \overline{T m}, .
$$

obtained from equations (46) and (48) in Section 21. We have seen that the quantity $b$ is likely to vary little with the density of a substance. Therefore over small regions of densities it may be taken as constant. The quantity $n$ of a substance we would expect to increase more rapidly than proportional to the density. If the substance behaved as a perfect gas $n$ would be proportional to the density, or inversely proportional to the volume according to Section 11. For densities at which the substance does not obey the law of a perfect gas the molecular attraction according to Section 25, has the effect of increasing $n$ above the value corresponding to the density. We may therefore suppose $n$ to consist of the sum of a number of terms, the first expressing the effect of the average minimum velocity (Section 16) on the value of $n$, and the other terms the effect of the increase in velocity produced by molecular attraction. The first term corresponds to supposing that the substance behaves as a perfect gas, and it therefore varies inversely as $v$ and may thus be written $C_{1} / v$, where $C_{1}$ is a constant which may be determined from the substance in the gaseous state. Of the remaining terms the first depends on the chance of each molecule coming under the influence of another molecule, which varies inversely as $v^{2}$, and the second term depends upon the chance of each molecule coming under the influence of two molecules, which varies 
inversely as $v^{3}$, and so on. Thus the sum of these terms may be written

$$
C_{2} / v^{2}+C_{3} / v^{3}+\ldots C_{2} / v^{2}
$$

and henee

$$
n=C_{1} / v+C_{2} / v^{2}+C_{3} / v^{3}+\ldots C_{2} / v^{2} .
$$

The quantities $C_{2}, C_{3}, \ldots C_{2}$ are evidently functions of $v$, for the ehance of two molecules coming close together will be influenced by the forees of attraction and repulsion of the surrounding molecules, and hence $C_{2}$ will depend on the molecular concentration, or on $v$, similarly in general the chance of any number of molecules coming close together will be influenced by the molecular forces of the surrounding molecules, and hence $C_{2}, C_{3}, \ldots C_{2}$ will be functions of $v$. But it will be evident on reflection that the variation of $n$ with $v$ in the foregoing equation will in the main be expressed by the inverse powers of $v$, in other words, the quantities $C_{2}, C_{3}, \ldots C_{2}$ are functions of $v$ which are not sensitive to variations of $v$. They are obviously functions of $T$ since the chance of a number of molecules coming close together will depend on their velocity of translation.

It follows from probability considerations that the terms $C_{2} / v^{2}, C_{3} / v^{3}, \ldots C_{z} / v^{2}$, decrease rapidly in magnitude in the order they stand. For the chance of each molecule coming close to another molecule and influencing its velocity of translation is equal to a fraction $p_{1}$, and the chance of each pair of molecules coming close to a third molecule and influeneing its velocity of translation is evidently therefore equal to $p_{1} p_{2}$, where $p_{2}$ is a fraction which is probably smaller than $p_{1}$, etc., and aecordingly

$$
p_{1}>p_{1} p_{2}>p_{1} p_{2} p_{3}>\ldots .
$$

If the quantities $C_{2}, C_{3}, \ldots C_{z}$ be expanded in inverse powers of $v$, an expression for $n$ is obtained which may be written

$$
n=C_{1} / v+C_{2 a} / v^{2}+C_{3 a} / v^{3} \ldots C_{2 a} / v^{2}
$$


where $C_{2 a}, C_{3 a}, \ldots C_{z a}$, are functions of $T$ only. It will be evident on reflection that since the first term of each expansion will be large in comparison with the remaining terms, the terms $C_{2 a} / v^{2}, C_{3 a} / v^{3}, \ldots C_{z a} / v^{2}$ decrease in magnitude in the order they stand, and probably rapidly.

In using equation (66) we may as a first approximation, from what has gone before, omit all the terms on the righthand side except the first two. Equation (65) then assumes the form*

$$
\frac{T \alpha}{\beta}=\left\{\frac{1}{v m_{a}} \sqrt{\frac{R T m}{3}}+\frac{C_{2}}{v^{2}}\right\} \frac{A v}{2(v-b)}, \ldots .
$$

since $C_{1}=\frac{1}{m_{a}} \sqrt{\frac{R T m}{3}}$ according to equation (21), where $A=5.087 \times 10^{-20} \sqrt{T m}$ according to equation (19), $\alpha$ denotes the coefficient of expansion and $\beta$ the coefficient of compression per dyne at the absolute temperature $T, v$ denotes the volume of a gram molecule of the substance and $b$ the apparent volume occupied by the molecules, $m_{a}$ denotes the absolute and $m$ the relative molecular weight of a molecule, and $R$ the gas constant whose value is given in Section 5 . If the distribution of molecular velocities is taken into account the right-hand side of the foregoing equation according to Maxwell's law has to be multiplied by 1.085 (Section 20).

The quantity $C_{2}$ we have seen is a function which varies little with $v$ and therefore over a small region of densities it may be taken as constant. Therefore on applying equation (67) at constant temperature to a substance at two densities not differing much from each other, two equations are obtained from which $C_{2}$ and $b$ may be determined. The values of $n$ corresponding to these two densities may then be obtained by equation (66) retaining two terms only of the right-hand side. Similarly the equation may be ${ }^{*}$ Not previously published and used. 
applied to another pair of densities not differing much from each other and from the other pair of densities, and the corresponding values of $n$ calculated. The values of $C_{2}$ and $b$ obtained in the two cases are not necessarily the same, though they will obviously differ very little from each other. Thus by a repeated application of equations (67) and (66) the values of $n$ and $b$ may be obtained for different densities of a substance. The accuracy of the results will evidently increase with the number of density values into which a given region of densities is divided. On the whole the method should give very good results.

On having obtained values of $n$ of a substance, the corresponding values of $V_{t}$, the total average velocity of a molecule, may be obtained by means of the equation

$$
V_{t}=\frac{3 n}{N_{c}}
$$

given in Section 18.

As an example of the foregoing investigation the values of $n$ and $V_{t}$ have been calculated for $\mathrm{CO}_{2}$ at $0^{\circ} \mathrm{C}$. corresponding to the pressures 100 and 200 atmospheres, which reduce the substance to the liquid state, the results being given in Table VIII.

\section{TABLE VIII}

\begin{tabular}{|c|c|c|c|c|}
\hline \multicolumn{5}{|c|}{$\mathrm{CO}_{2} \mathrm{AT} 0^{\circ} \mathrm{C}}$. \\
\hline $\begin{array}{c}p \text { in } \\
\text { atmos. }\end{array}$ & $\begin{array}{c}p+P_{n} \\
\text { in atmos. }\end{array}$ & $\begin{array}{c}r \text { in e.c. per } \\
\text { grm. mol. }\end{array}$ & $n$. & $V_{t} \frac{\text { cms. }}{\text { sec. }}$ \\
\cline { 1 - 4 } & 1 & $\ldots$ & $3.68 \times 10^{23}$ & $3.96 \times 10^{4}$ \\
100 & 2730 & 45.03 & $7.17 \times 10^{26}$ & $1.56 \times 10^{5}$ \\
200 & 4586 & 42.90 & $7.80 \times 10^{26}$ & $1.62 \times 10^{5}$ \\
\hline
\end{tabular}

The values of $P_{n}$ which were used to obtain the values of $T \alpha / \beta$ by equation (48), were deduced by the method of Section 21 from Amagat's experimental results on the 
relation between $v$ and $p$. The Table contains the values of $n$ and $V_{t}$ corresponding to a pressure of one atmosphere, in which case the substance is in the gaseous state, the values of the foregoing quantities in that case being given by equations (21) and (35). It will be seen that the value of $V_{t}$ is increased to about four times its original value as the pressure of the substance is increased from 1 to 100 or 200 atmospheres. This falls into line with the results of Sections 17, 21, and 25, according to which the effect of molecular attraction on the molecular velocity in a substance in the liquid state should be quite large. If the molecular forces had no effect on the magnitude of the motion of translation of the molecules, as is usually supposed, the value obtained for $C_{2}$ should have been zero, or at least very small. The result conclusively shows that the factor $V_{t} / V_{a}$ in equation (53) cannot be put equal to unity, and that the right hand side of Van der Waals' equation of state must be modified accordingly. The values of $n$ and $V_{t}$ obtained may be corrected according to Maxwell's law by dividing them by 1.085 .

The value of $b$, the apparent molecular volume of a gram molecule of molecules at the pressure 100 and 200 atmospheres is equal to 12.2 in cc., and thus is about $\frac{1}{4}$ of the total volume of a gram molecule of the substance. It is smaller than the volume at absolute zero, which according to Section 24 is approximately $\frac{1}{4}$ the volume $v_{c}$ at the critical point, or equal to $25.5 \mathrm{cc}$. This difference is due, as was explained in Sections 19 and 24, to the molecules getting nearer to each other at higher temperatures than the absolute zero through possessing kinetic energy of motion of translation.

If the value of the right hand side of the equation (67) is calculated for $\mathrm{CO}_{2}$ at a temperature $0^{\circ} \mathrm{C}$. and pressure of 50 atmos., using the foregoing values of the constant $b$ and $C_{2}$, the value of 2562 atmos. is obtained. This agrees 
well with the value of 2542 atmos. obtained directly for the left-hand side of the equation.

This investigation may be extended to a mixture of substances. In the case of a mixture of-say molecules $e$ and $r$, we would have

$$
\frac{T \alpha}{\beta}=2.543 \times 10^{-20}\left\{\frac{n_{e} v_{e}}{v_{e}-b_{e}^{\prime}} \sqrt{T m_{e}}+\frac{n_{r} v_{r}}{v_{r}-b_{r}^{\prime}} \sqrt{T m_{r}}\right\} .
$$

according to equations (41), (45), and (48), where $n_{e}$ denotes the number of molecules $e$ crossing a square $\mathrm{cm}$. from one side to the other in the mixture, and $n_{r}$ has a similar meaning with respect to the molecules $r$. Now we may write

$$
n_{r}=\frac{C_{1 r}}{v_{r}}+\frac{C_{2 r}^{\prime}}{v_{r}^{2}}, \ldots . . . .
$$

and

$$
n_{e}=\frac{C_{1 e}}{v_{e}}+\frac{C_{2 e}^{\prime}}{v_{e}^{2}}, . \quad . \quad . \quad . \quad .
$$

similarly as before, where $C_{1 r}$ and $C_{1 e}$ may be obtained from the substances $r$ and $e$ isolated and in the gaseous state. The resultant equation will then contain the four variables $C^{\prime}{ }_{2 r}, C^{\prime}{ }_{2 e}, b^{\prime}$, and $b_{r}^{\prime}$, which may be determined by applying the equation to the mixture at constant temperature corresponding to four densities not differing much from each other. Similarly a mixture of any number of constituents may be treated.

Another method may be used depending on variations of the relative concentration of the constituents of the mixture instead of variations of its density at constant relative concentration. Thus for $n_{e}$ and $n_{r}$ we may in this case write

and

$$
\left.\begin{array}{l}
n_{r}=a_{r}{ }^{\prime} N_{r}+a_{r}{ }^{\prime \prime} N^{2}+a_{r}{ }^{\prime \prime \prime} N_{r} N_{e} \\
n_{e}=a_{e}{ }^{\prime} N_{c}+a_{e}{ }^{\prime \prime} N^{2}{ }_{e}+a_{e}{ }^{\prime \prime \prime} N_{r} N_{e}
\end{array}\right\}
$$


where $a_{r}^{\prime}, a_{r}^{\prime \prime}, a_{r}^{\prime \prime \prime}, a_{e}^{\prime}, a_{e}^{\prime \prime}$, and $a_{e}^{\prime \prime \prime}$, are constants of which $a_{r}^{\prime}$ and $a_{e}^{\prime}$ may be determined according to Section 11 from the constituents isolated and in the gaseous state, and $N_{e}$ and $N_{r}$ denote the concentrations of the molecules $e$ and $r$ respectively. Similarly for $b_{e}^{\prime}$ and $b_{r}^{\prime}$ we may write

and

$$
\left.\begin{array}{c}
b_{e}{ }^{\prime}=k_{e}+N_{r} v_{e} k_{e r} \\
b_{r}{ }^{\prime}=k_{r}+N_{e} v_{r} k_{e r}
\end{array}\right\}, \quad \cdot \quad \cdot
$$

where $k_{e}, k_{r}$, and $k_{e r}$ are constants. It will be recognized that $k_{e}$ denotes the apparent volume due to the interaction of a molecule $e$ with the remaining molecules $e$, and $k_{r}$ has a similar meaning, while $N_{r} v_{e} k_{c r}$ denotes the apparent volume due to the interaction of a molecule $e$ with the molecules $r$, or vice versa, etc. On substituting from these equations in equation (68) an equation is obtained containing seven variables, which may be determined by applying at constant temperature the equation to seven different relative concentrations of the mixture differing little from each other.

In the case of a dilute solution we may evidently write

and

$$
\left.\begin{array}{l}
n_{r}=a_{r} N_{r} \\
n_{e}=a_{e} N_{e}
\end{array}\right\}, \quad \cdot . \cdot \cdot . \cdot
$$

where $a_{r}$ anu $a_{e}$ are constants. The foregoing process is thus much simplified in this case.

The foregoing method may be simplified by using the values of $b_{e}^{\prime}$ and $b_{r}^{\prime}$ calculated from the values of $b_{e}$ and $b_{r}$ of the constituents in the pure state; or we might make the assumption that $b_{r}{ }^{\prime}=b_{e}{ }^{\prime}$, which would probably introduce no serious error.

In Section 20 a method was given for finding approximate values of $b_{e}{ }^{\prime}$ and $b_{r}{ }^{\prime}$ from the values of $b_{e}$ and $b_{r}$ of the constituents in the pure state. Approximate values of 
$C_{2 r}^{\prime}$ and $C_{2 e}^{\prime}$ may similarly be obtained, and hence approximate values of $n_{r}$ and $n_{e}$ be obtained from equations (69) and (70). Let $C_{2}$, stand for $C_{2}$ in equation (67) when the substance consists of molecules $r$, and similarly let $C_{2 e}$ stand for $C_{2}$ when the substance consists of molecules $e$. The quantity $C_{2 r} / v_{r}^{2}$ then depends on the chance of each molecule in a pure substance of volume $v_{r}$ per gram molecule coming close to another molecule and changing its velocity of translation. This dependence may be exhibited in a certain way which is useful in dealing with mixtures. The chance of each molecule to get close to another molecule is proportional to the product of the concentrations of the molecules, or proportional to $N_{r} N_{r}$. Since $N_{r} m_{a r} v_{r}=m_{r}$, where $m_{a r}$ denotes the absolute and $m_{r}$ the relative molecular weight of a molecule, we have $N_{r}=\frac{\kappa}{v_{r}}$, where $\kappa$ denotes a constant. Thus the foregoing chance is proportional tc $1 / v_{r}^{2}$, and the resultant change in $n_{r}$ expressed by $C_{2 r} / v_{r}^{2}$. But if the gram molecule of molecuies $r$ contains molecules $e$ besides, a molecule $r$ may have its velocity changed through coming close to a molecule $e$. The chance of that happening to each molecule $r$ is proportional to the product of the concentrations of the molecules $r$ and $e$, or proportional to $N_{e} N_{r}$. This chance according to the foregoing results is proportional to $1 / v_{r} v_{e}$, where $v_{e}$ denotes the volume of the mixture containing a gram molecule of molecules $e$. The resultant change in the value of $n_{r}$ through the addition of molecules $e$ is therefore approximately given by $\frac{\sqrt{C_{2 r} C_{2} e}}{v_{r} v_{e}}$, where $C_{2 e}$ and $C_{2 r}$ refer to the molecules $e$ and $r$ in the pure state. The term $C^{\prime}{ }_{2 r} / v_{r}{ }^{2}$ in equation (69) consists of the sum of the foregoing two changes in $n_{r}$ brought about by molecular interaction, or

$$
\frac{C_{2 r}^{\prime}}{v_{r}^{2}}=\frac{C_{2 r}}{v_{r}^{2}}+\frac{\sqrt{C_{2 r} C_{2 e}}}{v_{r} v_{e}} .
$$


Similarly it can be shown that

$$
\frac{C_{2 e}^{\prime}}{v_{e}^{2}}=\frac{C_{2 e}}{v_{e}^{2}}+\frac{\sqrt{C_{2 r} C_{2 e}}}{v_{r} v_{e}} . \quad \cdot . .
$$

The values of $n_{r}$ and $n_{e}$ obtained from equations (69) and (70) by the help of equations (74) and (75) are likely to be fairly accurate. The accuracy may be. increased by using the third and higher power terms in the series for $n_{r}$ and $n_{e}$ in the equations.

The deduction of equation (65) used in this Section involves the use of the quantity intrinsic pressure. This quantity is therefore one of importance, and it will therefore be of interest to obtain further relations between it and other quantities.

30. An Equation Connecting the Intrinsic Pressure, Specific Heat, and other Quantities.

If $U$ denote the internal energy of a gram molecule of a substance, we have according to the Differential Calculus that

$$
\left(\frac{\delta U}{\delta T}\right)_{p}=\left(\frac{\delta U}{\delta v}\right)_{T}\left(\frac{\delta v}{\delta T}\right)_{p}+\left(\frac{\delta U}{\delta T}\right)_{v}
$$

The term on the left-hand side of the equation is the specific heat per gram molecule at constant pressure and was written $S_{i p l}$ in Section 13. For $U$ we may write

$$
U=U_{a}+u_{m}+\frac{3 R T}{2}, \ldots . \quad . \quad .
$$

as in Section 13, where $U_{a}$ denotes the potential energy of molecular attraction per gram molecule, $u_{m}$ the internal molecular energy, and $\frac{3 R T}{2}$ the kinetic energy of the molecules when situated at points at which the forces of the surrounding molecules neutralize one another, this energy 
being equal to the kinetic energy in the gaseous state according to Section 16. According to Section $21, \frac{\delta U_{a}}{\delta v}=P_{n}$ the intrinsic pressure, where $v$ denotes the volume of a gram molecule, and since $\frac{1}{v}\left(\frac{\delta v}{\delta T}\right)_{p}=\alpha$ the coefficient of expansion, the equation may be written

$$
S_{i p l}=\left(P_{n}+\left(\frac{\delta u_{m}}{\delta v}\right)_{T}\right) v \alpha+\left(\frac{\delta U_{a}}{\delta T}\right)_{0}+\left(\frac{\delta u_{m}}{\delta T}\right)_{0}+\frac{3 R}{2} .
$$

The quantity $\left(\frac{\delta u_{m}}{\delta v}\right)_{T}$ is very probably negligible in comparison with $P_{n}$, and may therefore be omitted from the equation. The quantity $\left(\frac{\delta U_{a}}{\delta T}\right)_{0}$ expresses the change in potential energy of molecular attraction of a substance with change in temperature at constant volume. It is very likely not zero in the case of a substance consisting of complex molecules, since according to Section 13 the relative distribution of the atoms in a molecule is changed with change in temperature, which would give rise to a change in the forces of attraction of the molecules upon each other. For the same reason the quantity $\left(\frac{\delta u_{m}}{\delta T}\right)_{0}$ is not likely to be zero in the case of a complex substance.

But if the molecules consist of atoms it is highly probable that these quantities and the quantity $\left(\frac{\delta u_{m}}{\delta v}\right)_{T}$ are zero. The equation then becomes*

$$
P_{n}=\frac{S_{i p l}-\frac{3 R}{2}}{v \alpha}, . . . . .
$$

and may thus be used to calculate $P_{n}$ in such a case.

${ }^{*}$ Not previously published. 
If the value of $P_{n}$ for a substance in the liquid state, which is mon-atomic in the gaseous state, is calculated by the foregoing equation, and it does not agree with that given by equation (48), it shows that the substance is polymerized in the liquid state. Thus, for example, equation (78) gives the value of $8.8 \times 10^{4}$ atmos. for $P_{n}$ for $H g$ at $0^{\circ} \mathrm{C}$., while equation (48) gives $1.25 \times 10^{4}$ atmos. It appears therefore that $\mathrm{Hg}$ is polymerized when in the liquid state. There is a good deal of other evidence pointing to the same conclusion.

Equation (78) may also give approximate values of $P_{n}$ in the case of substances consisting of complex molecules, since in most cases the differential quantities in equation (77) are relatively so small that they may be neglected. The difficulty of applying the equation to a substance arises principally through being dependent on a knowledge of the nature of the molecules, since the quantities $\frac{3 R}{2}$ and $v$ depend upon it. In the case of a partly polymerized substance a considerable error may therefore be introduced.

In the previous Sections we have considered various properties of substances which do not depend directly on the nature of the motion of a molecule and on its rapidity. There are, however, some properties that do, and which will be considered in the next Chapter. These properties are directly connected with the recurring nature of the path of a molecule, which exists according to the laws of probability. This introduces the idea of a recurring path of average length associated with a molecule, which will be discussed in the next Section, the results being introductory to the matter contained in the next Chapter. 
31. The Mean Free Path of a Moleculc under Given Conditions.

A molecule in a substance has its velocity continually changed in direction and magnitude through the influence of the surrounding molecules. At certain points along its path it may therefore satisfy certain conditions in respect. to the surrounding medium. The straight lines joining these points will be called the molecular free paths corresponding to the given conditions. These paths will obviously not have the same length, from probability considerations, but evidently the mean free path, the mean length of a large number of paths, should have a definite magnitude.

It is of importance and interest in this connection to calculate the probability that a molecule will pass over a distance $x$ without satisfying the conditions of the free path. This was first done by Clausius in connection with the mean free path of molecular collision. The investigation will be given a form here which applies to any kind of a free path.

The probability $P_{x}$ that a molecule will pass over the distance $x$ without satisfying the path conditions may be written

$$
P_{x}=\phi(x),
$$

and the probability that it will pass over the path $x+d x$ is therefore

$$
P_{x+d x}=\phi(x+d x)=\phi(x)+\phi^{\prime}(x) \cdot d x=P_{x}+\frac{d P_{x}}{d x} \cdot d x .
$$

But this is also according to the rules of probability equal to the product of the probability $P_{x}$ and the probability $P_{d x}$ that the molecule will pass over the distance $d x$ without satisfying the path conditions, that is

$$
P_{x} P_{d x}=P_{x}+\frac{d P_{x}}{d x} \cdot d x
$$


Now if $l$ denotes the mean free path of the molecule, the probability of it satisfying the path conditions in passing over the distance $d x$ is $d x / l$, and the probability of it not satisfying the path conditions is therefore

$$
1-\frac{d x}{l}=P_{d x}
$$

Hence the preceding equation may be written

$$
P_{x}\left(1-\frac{d x}{l}\right)=P_{x}+\frac{d P_{x}}{d x} \cdot d x,
$$

which on integrating gives

$$
P_{x}=C e^{-\frac{x}{l}},
$$

where $C$ denotes an arbitrary constant. Since the probability that the molecule will not satisfy the path conditions in passing over the distance $x=0$ is unity, it follows that $C=1$, and hence

$$
P_{x}=e^{-\frac{x}{l}}, \quad . \quad . \quad . \quad . \quad .
$$

which expresses the probability $P_{x}$ that the moiecule will pass over the distance $x$ without satisfying the free path condition, in terms of the quantities $x$ and the mean free path $l$.

The number $n_{x}$ of 100 molecules passing over the distance $x$ without satisfying the mean free path conditions is given as an illustration for a number of different values of $x$ in Table IX, where $x$ is expressed in terms of the mean free path $l$. It will be seen that a molecule rarely passes over a distance greater than its mean free path.

The probability that a molecule has a free path lying between the lengths $x$ and $x+d x$ is evidently

$$
P_{x}-P_{x+d x}=\frac{1}{l} e^{-\frac{x}{l}} \cdot d x, \quad . \quad . \quad .
$$

according to equation (79). 
TABLE IX

\begin{tabular}{|l|l||l|l||r|l|}
\hline$n_{x}$ & $x$. & $n_{x}$ & \multicolumn{1}{|c||}{$x$} & $n_{x}$ & $x$. \\
\hline 99 & $0.01 l$ & 78 & $0.25 l$ & 14 & $2 l$ \\
\cline { 1 - 2 } 9 & $0.02 l$ & 72 & $0.333 l$ & 5 & $3 l$ \\
90 & $0.1 l$ & 61 & $0.5 l$ & 2 & $4 l$ \\
82 & $0.2 l$ & 37 & $1.0 l$ & 1 & $4.5 l$ \\
\hline
\end{tabular}

In Section 21 the number of molecules $n$ crossing a plane one square $\mathrm{cm}$. in area in a substance was investigated. In connection with the investigation of this Section it is of interest to determine the probability that the path of a molecule crossing the plane has a length lying between $x$ and $x+d x$. It is evident that on moving the plane from one position to another parallel to itself its chance of cutting a free path of length $x$ will be proportional to the value of $x$. Therefore, since a molecule in its migrations has different consecutive paths the probability of the plane cutting one of length $x$ is equal to the ratio of $x$ to the sum of all of the different paths of the molecule divided by their number. The latter quantity is equal to the mean free path $l$, and hence the ratio is equal to $x / l$. This probability must be multiplied by the probability that the molecule has a path lying between $x$ and $x+d x$ which is given by equation (80). Therefore the probability $P_{c}$ that the free path of a molecule which lies between the lengths $x$ and $x+d x$ cuts the plane is

$$
P_{c}=\frac{x}{l^{2}} e^{-\frac{x}{l}} \cdot d x .
$$

It should be noted that the foregoing investigation is independent of the nature of the conditions defining the free path of a molecule and that therefore these conditions may be given any form we please.

It will be of interest to compare the square of the aver- 
age path $l$, or $l^{2}$, with the mean of the squares of the different paths. The latter quantity is given by

$$
\int_{0}^{\infty} \frac{x^{2}}{l} e^{-\frac{x}{l}} \cdot d x=2 l^{2},
$$

by the help of equation (80). Thus the latter quantity is double the former.

We are in a position now to develop formulæ for the quantities viscosity, conduction of heat, and diffusion, which depend directly on the nature of the motion of a molecule, in terms of quantities referring to the nature of this motion, and other quantities. In order that the formulæ may be given forms applying to different states of matter I have introduced new definitions in connection with the path of a molecule under different conditions, which make no reference to molecular collision, and apply to all states of matter. They lead to formulæ involving quantities usually not considered in treatises on the Kinetic Theory of Gases. These give interesting information as to how the various molecular properties give rise to, or modify, the viscosity, conduction of heat, and diffusion, of a substance. I have previously made an attempt to obtain formulæ for the foregoing properties of matter independent of the idea of molecular collision*, and finally developed the results given in this book, which, however, differ considerably from the previous results, and are therefore more or less new.

*Phil. Mag., July, 1912, pp. 101-118. 


\section{CHAPTER III}

QUANTITIES WHICH DEPEND DIRECTLY ON THE NATURE OF MOLECULAR MOTION

\section{The Coefficient of Viscosity of a Substance.}

A solid in contact with a liquid or gas at rest experiences a resistance when displaced depending on the nature of the gas or liquid, the resistance remaining constant when the motion is uniform. This arises through matter being carried along by the surface of the solid. This property of liquids and gases is known as viscosity. It can be shown that it primarily arises from the fact that the molecules of a substance undergo a rapid motion of translation. Thus suppose that $n$ molecules per square $\mathrm{cm}$. per second strike the lower surface of the solid $A$ shown in Fig. 10, which is moving with a velocity $V_{1}$ parallel to its surface. If the average momentum parallel to the surface of the solid of the molecules which per second are about to collide with the solid, is $n V_{2} m_{a}$, the momentum after collision is that corresponding to the velocity of motion of the solid, or equal to $n V_{1} m_{a}$, if the colliding molecules assume the velocity of the solid, and thus the solid imparts a momentum equal to $n m_{a}\left(V_{1}-V_{2}\right)$ to the substance per second. According to the equation $F t=m_{a} V$, this momentum is equal to the force $F$ that has to be applied to the solid per square $\mathrm{cm}$. of the surface in order to maintain uniform motion, or $F=n m_{a}\left(V_{1}-V_{2}\right)$. The layer of liquid in the immediate vicinity of the surface is accordingly set in motion, and this. 
motion is communicated to the next layer, and so on. The whole liquid is thus set in motion along planes parallel to the surface of the solid, the velocity of motion decreasing with the distance from the surface.

It follows from the above equation that the force necessary to maintain a velocity $V_{1}$ of the solid depends upon the value of $V_{1}-V_{2}$, which is proportional to the velocity

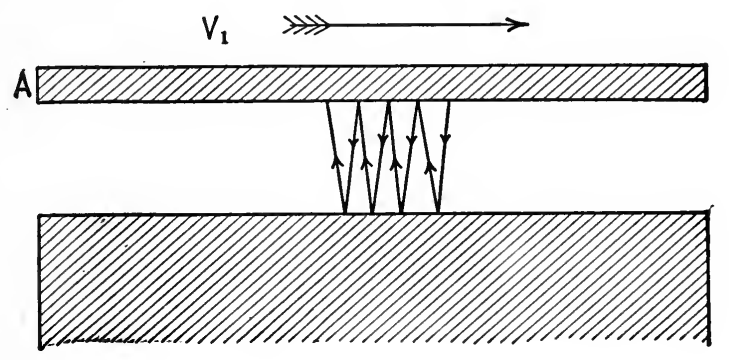

Fig. 10.

gradient of the liquid in the immediate vicinity of the surface, and thus the foregoing equation may be written

$$
F=n m_{a} K \cdot \frac{\delta V}{\delta x} \cdot \cdot \cdot \cdot \cdot \cdot \cdot
$$

where the distance $x$ is measured at right angles to the surface of the solid, and $K$ denotes a constant. If the velocity gradient is unity the corresponding force $F$ is called the coefficient of viscosity, and will be denoted by $\eta$. Hence if a plane moving with a velocity $V_{1}$ is at a distance $d$ from a fixed plane, the force $F$ that has to be applied per square $\mathrm{cm}$. of the moving plane may be written

$$
F=\eta V_{1} / d,
$$

which accordingly becomes equal to $\eta$, when the velocity gradient is unity. The magnitude of the viscosity may be 
smaller than indicated by the foregoing investigation through the molecules on striking the moving solid not acquiring exactly its velocity. This effect is known as slipping of the fluid over the surface of contact of the solid. Experiment has shown that there is no appreciable slipping in the case of liquids, and this also holds in the case of gases, except at very low pressures. This point will be further discussed in Section 34. It will not be difficult to see that if the moving solid exerted a repulsion upon the molecules of the surrounding fluid this would have the effect of tending to turn back the molecules approaching it without giving to them a motion parallel to that of the surface of the solid. In that case slipping would occur. It is not improbable that the double layer of electricity which exists at the interface of a fluid and solid may give rise to some slipping in this way.

The coefficient of viscosity of a substance is usually obtained in practice by measuring the volume $v_{1}$ of the substance which passes per second through a narrow tube of radius $r$ and length $L_{1}$ under the action of a pressure $p$. If the substance escapes from the tube with a velocity which is negligible in comparison with the velocity that would be obtained with a tube of large radius, the value of $\eta$ according to Poiseuille is given by

$$
\eta=\frac{\pi p r^{4}}{8 v_{1} L_{1}}
$$

If, however, the escaping liquid possesses an amount of kinetic energy which eannot be neglected the term $\frac{\rho V^{\prime}}{8 \pi L_{1}}$ has to be subtracted from the right-hand side of the foregoing equation where $V^{\prime}$ denotes the velocity of the escaping fluid and $\rho$ its density.

The viscosity of a substance depends not only on the velocity of translation of a molecule, but on the nature of 
the motion, and it will therefore be necessary to define a quantity connected with the path described by a molecule.

33. The Viscosity Mean Momentum Transfer Distance of a Molecule in a Substance.

Consider a viscous medium in motion parallel to the plane $A B$ which is kept at rest, as shown in Fig. 11. Let $a b c$
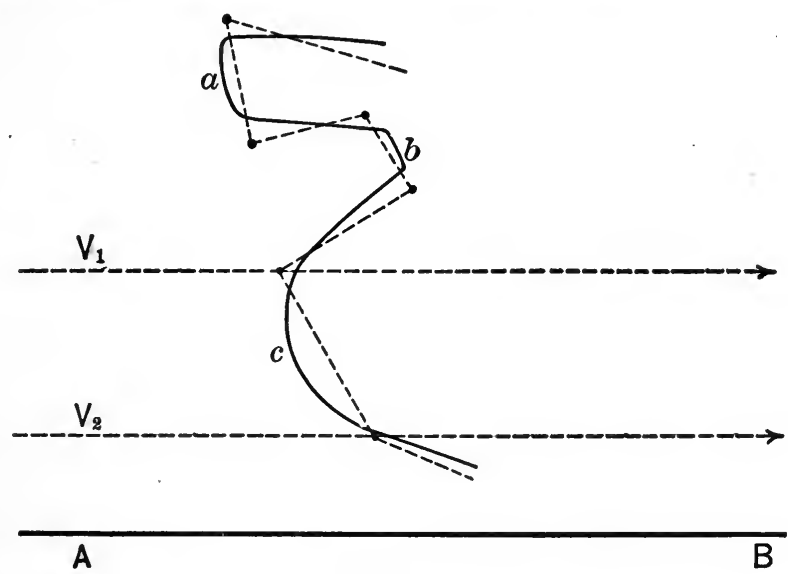

FIG. 11.

denote the path of a molecule moving towards the plane $A B$, and which therefore passes progressively into slower moving portions of the substance. The molecule during its course loses momentum, continually but in varying amounts to the medium parallel to the direction it is moving, through the interaction of the molecules due to their molecular forces of attraction and repulsion and their molecular volume. Similarly a molecule passing in the opposite direction continually acquires momentum from the medium. The medium evidently does not acquire or lose momentum only at isolated points. We may suppose, however, that 
the effect is equivalent to a row of points being associated with the molecular path, not necessarily lying on it, at which only the medium acquires or loses momentum. Further let us suppose that the momentum lost by the medium at a point is equal to $V_{2} m_{a}$, where $V_{2}$ denotes the velocity of the medium at the point, and $m_{a}$ the molecular weight of a molecule, this momentum being absorbed by the migrating molecule, while the momentum gained by the medium at the same point is equal to $V_{1} m_{a}$, where $V_{1}$ denotes the velocity of the medium at the preceding point, this momentum being abstracted from the migrating molecule. The distance between two consecutive points will be called the momentum transfer distance corresponding to the path of the migrating molecule.

The moiecule thus appears to abstract the momentum $V_{1} m_{a}$ from a point in the medium and to transfer it to a consecutive point, while at the latter point it abstracts the momen $u$ um $V_{2} m_{a}$ from the medium and transfers it to a point consecutive to the latter, and so on. The positions of these points, and their number per unit length of path, are to a certain extent arbitrary, as will easily be recognized, and it is therefore necessary to introduce conditions which will render the lines joining them quite definite. One condition that the points obviously have to satisfy is that the flow of momentum in the substance should be uniform everywhere. But this condition alone is not sufficient. Let us therefore impose the two additional conditions that the number $n$ of molecular paths crossing a square $\mathrm{cm}$. from one side to the other shall be equal to the number of transfer distances passing through the square cm., and that all directions of a transfer distance in space are equally probable. We will see in the next Section that these conditions completely determine the distribution of the points in question. According to Section 31 the various transfer distances corresponding to these points would not be of 
the same magnitude, but grouped about a mean transfer distance $l_{\eta}$ according to a general law whose form was obtained.

It should be noted that according to the definition of a transfer distance the length of path of a molecule between two points is equal to the sum of the corresponding momentum transfer distances. The transfer distances will therefore be associated with the molecular path in the way shown in Fig. 11.

A migrating molecule is evidently continually under the action of the resultant force arising from the forces of the

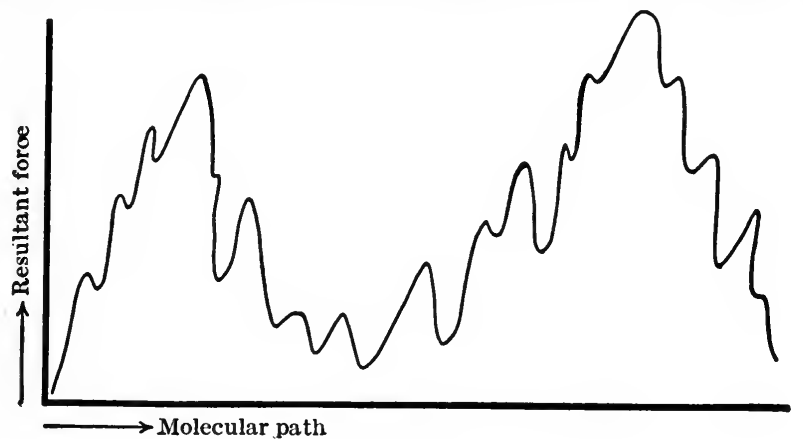

Fig. 12.

surrounding molecules. This resultant force is continually changing in direction and magnitude. It will therefore pass through a series of maxima and minima of different magnitudes, which may graphically be illustrated by the curve in Fig. 12. The largest maxima correspond to the closest possible approach of two molecules, while the other maxima correspond to various distances of approach, whose effect is modified by the situation of the surrounding molecules. Thus some of the maxima and minima will have very small though finite values. This holds for a gas as well as for a liquid. The theoretical points at which a molecule is supposed to absorb momentum from the medium or transfer 
momentum to it evidently therefore do not necessarily correspond to maxima or minima values of the resultant force acting on the molecule, and besides on account of the small values of some of the maxima and minima several of these are likely to be situated between two such consecutive points. These considerations show that if molecular forces exist between molecules the momentum free path cannot be defined with respect to molecular collision, but must be defined along such lines as developed in this Section.

It will not be difficult to see that even if the molecules consisted of hard elastic spheres not surrounded by fields of force the average distance between two consecutive collisions in a gas need not be equal to the length of the average momentum transfer distance as just defined. In fact it can be shown that these distances are not equal to one another. But the opposite is usually tacitly implied when the mean free path of a molecule in a gas is defined according to molecular collision and then used to obtain a formula for the viscosity of a gas in the usual way. Of course, some mathematicians have begun to realize this and endeavored to determine the appropriate factor that has to be associated with the mean free path in the formula. But this cannot lead to anything definite because the interaction between molecules is largely, if not altogether, due to the existence of the molecular forces.

A general formula for the viscosity of a substance in the gaseous, liquid, and intermediary states of matter, in terms of the momentum transfer distance will now be developed and applied to the facts.

\section{Formula for the Viscosity in Terms of Other Quantities.}

Let us consider as before a substance moving in planes parallel to a plane $A B$ which is at rest, as is shown in Fig. 
13. Suppose that $n$ molecules per square $\mathrm{cm}$. cross the plane $E F$ from one side to the other. The migration of these molecules gives rise to a transference of momentum across the plane from top to bottom which per square $\mathrm{cm}$. is numerically equal to the coefficient of viscosity if the velocity gradient is equal to unity. The expression for this momentum we will now proceed to find. Consider the momentum transfer distances which cut the plane $E F$ and are inclined at the angle $\theta$ with a perpendicular to the plane. If we suppose for purposes of calculation that these distances start from the same point which we will suppose lies in the plane $E F$, it follows from the figure, which shows the sec-
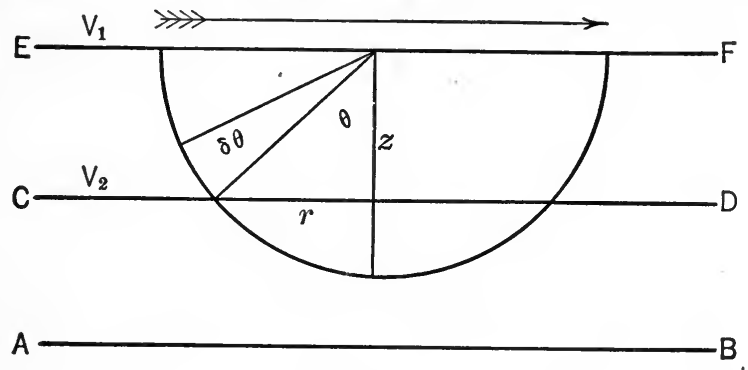

Fig. 13.

tion of a hemisphere of radius $z$ made by a plane at right angles to the plane $E F$, that the number of these distances is equal to

$$
n \frac{2 \pi r z \cdot d \theta}{2 \pi z^{2}},
$$

where $z$ denotes the length of one of these distances, $r=z \sin \theta$, and this number is therefore equal to

$$
n \sin \theta \cdot d \theta .
$$

If $n_{z}$ denote the number of the foregoing distances whose lengths lie between $z$ and $z+d z$, we have

$$
n_{z}=n \sin \theta \cdot d \theta \cdot \frac{z}{l_{\eta}^{2}} e^{-\frac{z}{l_{\eta}}} \cdot d z,
$$


by the help of equation (81), where $l_{\eta}$ denotes the mean momentum transfer distance. The molecules corresponding to these distances abstract the momentum $n_{2} m_{a} V_{1}$ from the plane $E F$, which moves with the velocity $V_{1}$, and transfers it to the plane $C D$ which moves with the velocity $V_{2}$. From considerations of equilibrium it follows that an equal number of molecules migrate in the opposite direction. These abstract the momentum $n_{2} m_{a} V_{2}$ from the plane $C D$ and transfer it to the plane EF. Thus a transference of momentum across the plane takes place which is equal to

$$
n_{2} m_{a}\left(V_{1}-V_{2}\right) \text {. }
$$

On taking the velocity gradient equal to unity we have

$$
\frac{V_{1}-V_{2}}{z \cos \theta}=1,
$$

and the foregoing expression accordingly becomes

$$
n_{z} m_{a} z \cos \theta
$$

To obtain the total momentum transferred per square $\mathrm{cm}$. across the plane $E F$, or the coefficient of viscosity $\eta$, the foregoing expression on substituting for $n_{z}$, must be integrated from 0 to $\infty$ with respect to the transfer distance $z$, and from 0 to $\pi / 2$ with respect to the angle $\theta$, giving

Since

$$
\eta=n m_{a} \int_{0}^{\frac{\pi}{2}} \int_{0}^{\infty} \cos \theta \cdot \sin \theta \cdot \frac{z^{2}}{l_{\eta}^{2}} e^{-\frac{z}{l_{\eta}}} \cdot d \theta \cdot d z .
$$

and

$$
\int_{0}^{\frac{\pi}{2}} \cos \theta \cdot \sin \theta \cdot d \theta=\frac{1}{2},
$$

$$
\int_{0}^{\infty} \frac{z^{2}}{l_{\eta}^{2}} e^{-\frac{z}{l_{\eta}}} \cdot d z=2 l_{\eta}
$$


the foregoing equation becomes

$$
\eta=n m_{a} l_{\eta}, \quad \cdot \quad \cdot \quad \cdot \quad \cdot \quad \cdot \quad \cdot
$$

where $n$ denotes the number of molecules of absolute molecular weight $m_{a}$ crossing a plane of one square $\mathrm{cm}$. from one side to the other per second, and $l_{\eta}$ denotes the mean momentum transfer distance.

The foregoing equation in the form it stands is mainly of use in determining the value of $l_{\eta}$, since $\eta$ may be measured directly, and $n$ determined by the method described in Section 29.

The quantity $l_{\eta}$ may be expressed in terms of other quantities. Let us suppose that the substance is represented by another substance possessing the same viscosity and expansion pressure, but whose molecules possess no volume of the kind represented by the symbol $b$ (Section 19). This is possible since the law of molecular attraction of the representative substance is initially left arbitrary, and may therefore be given a form that the foregoing conditions are satisfied. The law may evidently involve as many arbitrary constants as we please. The viscosity of the representative substance is given by

$$
\eta=n^{\prime} m_{a} l_{\eta}^{\prime}
$$

where $n^{\prime}$ and $l^{\prime}{ }_{\eta}$ have meanings corresponding to $n$ and $l_{\eta}$ in equation (83). Since the representative substance has the same expansion pressure as the original substance, and the molecules of the former substance have no volume, we have from equations (38) and (45) that

$$
n^{\prime}=n \frac{v}{v-b}=\frac{P_{n}+p}{A / 2}
$$

where the quantities $n, v, b, P_{n}, p$, and $A$, refer to the original 
substance, and the preceding equation may therefore be written

$$
\eta=\frac{v}{v-b} n m_{a} l_{\eta}^{\prime}=\frac{\left(P_{n}+p\right)}{A / 2} m_{a} l_{\eta}^{\prime} . \quad . .
$$

Each quantity in this equation, except $l_{\eta}^{\prime}$, may be determined directly or indirectly by experiment, and the latter quantity is therefore determined by the equation. The fact that the equation contains a variable which does not refer directly to the original substance shows that it may be represented by another substance under the conditions stated.

On comparing the foregoing equation with equation (83) we see that

$$
l_{\eta}=\frac{v}{v-b} l_{\eta}^{\prime}, \quad . \quad . \quad . \quad . .
$$

and thus $l_{\eta}^{\prime}$, the mean transfer distance in the representative substance, is evidently an inferior limit of $l_{\eta}$. We will see later that $l_{\eta}$ does not differ appreciably from $l_{\eta}^{\prime}$ except when the density of the substance corresponds to that of the liquid state.

It is often of interest to compare the distance of separation $d$ of the molecules in a substance with the quantity $l^{\prime}{ }_{\eta}$. We have immediately $d=\frac{w_{1}}{v^{1 / 3}}$, where $w_{1}$ is a constant. For $l^{\prime}{ }_{\eta}$ we may write $l_{\eta}^{\prime}=w_{2} v$, where $w_{2}$ is a function of $v$ which becomes a constant when the substance is in the gaseous state. The ratio of $l^{\prime}{ }_{\eta}$ to $d$ is then given by

$$
\frac{l_{\eta}^{\prime}}{d}=v^{2 / 3} w_{3}, \quad . \quad \cdot \quad \cdot \cdot \cdot
$$

where $w_{3}$ is a function of $v$ which becomes a constant when the substance is in the gaseous state.

The quantity $l_{\eta}^{\prime}$ in equation (84) may be expressed in 
terms of quantities which may be taken to refer to the original substance. If the molecules in the representative substance were devoid of forces of attraction and repulsion which extend beyond a small distance from each molecule, $l_{\eta}^{\prime}$ would be inversely proportional to the chance of one molecule encountering another, and thus inversely proportional to the concentration of the molecules, or proportional to the volume $v$, and thus we may write

$$
l_{\eta}^{\prime}=\kappa_{\eta} v
$$

under these conditions, where $\kappa_{\eta}$ is a constant at constant temperature. The existing forces of attraction and repulsion have the effect of modifying the interaction of two molecules, in which case $l_{\eta}^{\prime}$ would not vary according to the foregoing equation. The effect of the molecular forces may be expressed by introducing a factor into the right-hand side of the foregoing equation, which is determined from the following considerations. The chance of a migrating molecule coming under the influence of another molecule, and the pair of interacting molecules coming under the influence of a third molecule, is proportional to the square of the molecular concentration, or inversely proportional to $v^{2}$; the chance of a migrating molecule coming under the influence of another molecule, and the pair of interacting molecules coming under the influence of two molecules, is inversely proportional to $v^{3}$; and so on. Thus the factor in question consists of the series

$$
\left(1+\frac{\phi^{\prime}}{v^{2}}+\frac{\phi_{\eta}^{\prime \prime} \eta}{v^{3}}+\ldots\right)=1+\Phi_{\eta}, \ldots .
$$

where $\phi_{\eta}^{\prime} / v^{2}$ expresses the average effect of a single molecule on a pair of interacting molecules, $\phi^{\prime \prime}{ }_{\eta} / v^{3}$ the effect of a pair of molecules, and so on, while $\Phi_{\eta}$ denotes the sum of the series involving $v$. The quantities $\phi_{\eta}^{\prime}, \phi^{\prime \prime}{ }_{\eta}, \ldots$, are 
functions of $v$ and $T$, which, however, probably vary little with $v$. For $l_{\eta}$ and $l_{\eta}^{\prime}$ we have therefore the expressions

$$
l_{\eta}=l_{\eta}^{\prime} \frac{v}{v-b}=\frac{\kappa_{\eta} v^{2}}{v-b}\left\{1+\Phi_{\eta}\right\}
$$

and

$$
l_{\eta}^{\prime}=\kappa_{\eta} v\left\{1+\Phi_{\eta}\right\}
$$

The interpretation of equation (88) is interesting. When two molecules interact the process is modified by the surrounding molecules, which effect may be called molecular interference. The value of $b$ depends on molecular interference, but probably only to a small extent. But the quantity $\Phi_{\eta}$ depends entirely on it, and this quantity may therefore be called the interference function in viscosity. The effect of molecular interference on the value of $l_{\eta}$, according to equation (88), is expressed by the quantities $b$ and $\Phi_{\eta}$, but mainly by $\Phi_{\eta}$, in other words, the effect of molecular interference on the value of $l_{\eta}$ which is not included in the value of $b$ is represented by the interference function $\Phi_{\eta}$, which may now be used without referring to the representative substance.

Equation (83) referring to the original substance may now be written in the forms

$$
\begin{aligned}
& \eta=n m_{a} \kappa_{\eta} \frac{v^{2}}{v-b}\left\{1+\Phi_{\eta}\right\}, \ldots . . \\
& \eta=\frac{1}{3} V_{\imath} m \kappa_{\eta} \frac{v^{-}}{v-b}\left\{1+\Phi_{\eta}\right\}, \ldots . .
\end{aligned}
$$

and

$$
\eta=\frac{2\left(P_{n}+p\right) m_{a} \kappa_{\eta} v}{A}\left\{1+\Phi_{\eta}\right\} .
$$

by means of equations (35), (38), (45), and (88), remembering that $N_{c} m_{a} v=m$, the molecular weight in terms of that of the hydrogen atom. 
The values of $n, b$, and $V_{t}$, the number of molecules crossing a square $\mathrm{cm}$., the apparent molecular volume, and the total average velocity, respectively, may be determined by the method described in Section 29 , and the value of the intrinsic pressure $P_{n}$ by the method described in Section 21. The calculated values of $n, b$, and $V_{t}$ depend on the assumed distribution of molecular velocities, ${ }^{*}$ and may be corrected according to Maxwell's law, if desired, in the way described. The value of $A$ in equation (92) not corrected for the distribution of molecular velocities is given by

$$
A=5.087 \times 10^{-20} \sqrt{\mathrm{Tm}}
$$

according to Section 11. If corrected according to Maxwell's law the value has to be multiplied by $\sqrt{\frac{3 \pi}{8}}$, or 1.085 . It will appear, however, from Sub-section $a$ that the calculated value of $\kappa_{\eta}$ similarly corrected involves the factor 1.085, and that therefore the uncorrected values of $\kappa_{\eta}$ and $A$ may be used in equation (92). It follows then that in equations (90) and (91) the uncorrected values of $\kappa_{\eta}, n, b$, and $V_{t}$ may be used, since $p$ is independent of molecular motion (Section 8$), P_{n}$ is obviously so by nature, and therefore the factor $\kappa_{\eta} / A$ in equation (92) is represented in the preceding equations by factors involving $\kappa_{\eta}, n, b$, and $V_{t}$.

The value of $\kappa_{\eta}$ for a given substance is immediately determined by applying equation (92) to the substance in the perfectly gaseous state, which corresponds to $v=\infty$, and therefore to $P_{n}=0$ and $\Phi_{\eta}=0$. It may be noted that according to equations (85), (88), and (89) we have $l_{\eta}=$ $l_{\eta}^{\prime}=\kappa_{\eta} v$ when the original and representative substances are in the perfectly gaseous state, and the value of $\kappa_{\eta}$ is therefore the same for both substances. It evidently destate.

* This distribution refers to molecules in the perfectly gaseous 
pends on the molecular forces and the molecular volume of the molecules of the original substance.

It will be evident on reflection that the quantities $n, V_{l}, b, P_{n}$, and $p$ are affected to a certain extent by the influence of the molecules of the substance on two molecules while they interact, or are affected by molecular interference. The quantity $\Phi_{\eta}$, or the interference function, therefore, represents the effect of molecular interference on the viscosity of the substance which is not represented by the foregoing quantities in equations (90), (91), and (92). Thus we may now use these equations without referring to the representative substance.

The series for the interference function $\Phi_{\eta}$ which occurs in the foregoing equations may be given a simpler form which holds approximately. The probability of a migrating molecule coming under the influence of another molecule, and the pair of interacting molecules coming under the influence of $y$ molecules, is much greater than the probability of their coming under the influence of $y+1$ molecules, and thus the terms in this series stand in the order of magnitude $\frac{\phi_{\eta}^{\prime}}{v^{2}}>\frac{\phi^{\prime \prime}{ }_{\eta}}{v^{i}}>\ldots$, and are likely to decrease in magnitude very rapidly. As a first approximation we may therefore retain only the term $\frac{\phi^{\prime}}{v^{2}}$, or replace the series by the term $\frac{\Phi^{\prime}}{v^{x}}$, where $x$ would differ little from 2.

It is instructive to apply equation (91) to a hypothetical substance whose molecules consist of perfectly elastic spheres not surrounded by fields of force. The value of $V_{t}$ then corresponds to that when the substance is in the gaseous state at the same temperature according to Section 19, and $\Phi_{\eta}$ represents the interference by actual contact of one or more molecules with two molecules about to collide or undergoing collision. Since in all cases $\Phi_{\eta}$ in the representa- 
tive substance represents an effect due to molecular attraction, the quantity will have the same sign in this particular case as found in practice, namely positive according to Subsection $(d)$. The effect of the true molecular volume according to equation (91) would thus be to increase the viscosity by means of the positive apparent volume $b$, and the molecular interference represented by $\Phi_{\eta}$, to both of which it would give rise.

The number $N_{t}$ of times the mean transfer distance associated with the path of a molecule is passed over per second is a quantity of interest. Since the length of molecular path between two points is equal to the sum of the momentum transfer distances, we have immediately

$$
N_{t}=\frac{V_{t}}{l_{\eta}}=\frac{V_{t}^{2} \rho}{3 \eta}, \ldots . . . .
$$

by the help of equations (83) and (35), where $V_{\imath}$ denotes the total average velocity of a molecule, and $N_{c} m_{a}=\rho$ the density of the substance. The value of $V_{t}$ may be determined by the method of Section 29 in the case of a liquid, or gas not obeying Boyle's law.

Applications of the foregoing equations will now be given.

(a) On applying equation (90) to the perfectly gaseous state, which corresponds to $v=\infty$, it becomes

$$
\eta=n m_{a} \kappa_{\eta} v=\kappa_{\eta} \sqrt{\frac{R T m}{3}}, . . . .
$$

by the help of equation (21). It follows from this equation that, since $\kappa_{\eta}$ is constant at constant temperature, $\eta$ is independent of the density of the gas. This remarkable result was first deduced by Maxwell,* and has been amply confirmed by experiment. The deviations that have been obtained are due in part to the conditions of the experi* This deduction depends on the method of molecular collision. 
ment not conforming to the conditions underlying the deduction of the above equation. Thus for example, the result is found to break down completely at extremely low pressures. Now it will be evident from an examination of the deduction of the foregoing equation that it will hold only so long as $l_{\eta}$ is small in comparison with the thickness of the moving layer of gas. The equation would therefore begin to break down when the value of $l_{\eta}$ becomes comparable with the linear dimensions of the apparatus used for measuring the viscosity.

There is, however, another cause operating giving rise to a deviation of equation (94) from the facts at low pressures. The effect of the slipping of the gas along the moving plane on the value of the viscosity increases as the pressure decreases. Thus let $V_{a}$ denote the velocity of the moving plane, $V_{b}$ the component velocity of a molecule parallel to the plane before striking it, and $V_{c}$ the component velocity of rebound parallel to the plane. Then if the molecule undergoes slipping along the plane during rebound $V_{a}>V_{c}>V_{b}$. The viscosities $\eta$ and $\eta_{1}$ when there is no slipping and slipping respectively are proportional to the velocity gradients in the gas, and hence proportional to $V_{a}$ and $V_{c}$ the corresponding velocities of the molecules on rebound, which gives $\eta / \eta_{1}=V_{a} / V_{c}$. Now the difference between $V_{a}$ and $V_{b}$ increases with increase of the mean momentum transfer distance, or distance of the layer of gas from which the molecule comes, and thus increases with decrease of pressure. The difference between $V_{a}$ and $V_{c}$ therefore also increases with decrease of pressure, which increases the value of the ratio $\eta / \eta_{1}$ according to the foregoing equation. This corresponds to a decrease of $\eta_{1}$, and thus the effect of slipping becomes the greater the lower the pressure.

The dynamical mechanism underlying the result that the viscosity of a gas is independent of its density may be 
illustrated by the following considerations. A molecule transfers a certain amount of momentum to the gas at the end of each transfer distance on migrating at right angles to the motion of the gas in the direction of the decrease of motion. If the concentration of the gas is halved the length of each transfer distance is doubled, while the momentum transferred at the end of each transfer distance is also doubled since the velocity gradient of the gas remains the same. Since a change in molecular concentration of a gas does not alter the molecular velocities, the momentum transferred per second by a molecule moving between two parallel plates of material one of which is at rest while the other moves parallel to itself is in the latter case double that in the former. But since the number of molecules per cubic $\mathrm{cm}$. available for momentum transference in the former case is half the number in the latter, the total momentum transferred is in each case the same, or the viscosity of the gas has not been altered by altering its density.

The quantity $\kappa_{\eta}$ is a function of the temperature. This is shown by the calculated values of $\kappa_{\eta}$ contained in Table $\mathrm{X}$ for a number of gases at different temperatures. These values are not corrected for Maxwell's law of distribution of velocities since $n$ given by equation (21) and substituted in equation (90) was not thus corrected. If this correction is carried out the values in the Table have to be multiplied by $\sqrt{\frac{3 \pi}{8}}$, or 1.085 . It will be seen that the values of $\kappa_{\eta}$ increase with increase of temperature in the case of each gas mentioned in the Table, and this was found to hold for every other gas that was examined. This indicates, since the mean momentum transfer distance $l_{\eta}$ is equal to $\kappa_{\eta} v$, that the distance over which a migrating molecule transfers momentum in a gaseous medium is increased by an increase of temperature. The reason probably is that the greater the velocity of two molecules approaching and 
receding from each other the shorter is the time they are under the influence of each other's attraction, and therefore the smaller is the change in momentum imparted to each other. Large changes in momentum will therefore take place only for close distances of approach, and these will therefore take place less frequently along the path of a molecule with increase of temperature.

\section{TABLE $\mathrm{X}$}

\begin{tabular}{|c|c|c|c|c|c|}
\hline \multicolumn{3}{|c|}{ MERCURY. $\quad m=200.4$} & \multicolumn{3}{|c|}{ KRYPTON. $\mathrm{m}=81.8$} \\
\hline$t^{\circ} \mathrm{C}$ & $\eta 107$ & $\kappa_{\eta} 10^{10}$ & $t^{\circ} \mathrm{C}$ & $\eta 10^{7}$ & $\kappa_{\eta} 10^{10}$ \\
\hline 300 & 5320 & 2.98 & 0 & 2334 & 2.97 \\
\hline 380 & 6560 & 3.94 & 100 & 3063 & 3.33 \\
\hline \multicolumn{3}{|c|}{ ARgon. $\quad \mathrm{m}=39.9$} & \multicolumn{3}{|c|}{ HYDROGEN. $\quad \mathrm{m}=2$} \\
\hline-183.2 & 735.6 & 2.62 & -194.9 & 374.2 & 5.69 \\
\hline 0 & 2104 & 3.83 & 0 & 822 & 6.69 \\
\hline 183.3 & 3243 & 4.57 & 302 & 1392 & 7.81 \\
\hline \multicolumn{3}{|c|}{ Ether. $\quad \mathrm{m}=74$} & \multicolumn{3}{|c|}{ ETHYL CHLORIDE. $\mathrm{m}=64.5$} \\
\hline 0 & 689 & .92 & 0 & 935 & 1.34 \\
\hline 100 & 967 & 1.11 & 157.3 & 1440 & 1.64 \\
\hline 212.5 & 1234 & 1.24 & 240.6 & 1714 & 1.79 \\
\hline \multicolumn{3}{|c|}{ Carbon dioxide. $\mathrm{m}=44$} & \multicolumn{3}{|c|}{ ETHyLENe. $\quad \mathrm{m}=28$} \\
\hline-21.5 & 1278 & 2.31 & -21.5 & 891 & 2.02 \\
\hline 100 & 1972 & 2.92 & 99.25 & 1278 & 2.38 \\
\hline 302 & 2682 & 3.21 & 302 & 1826 & 2.73 \\
\hline \multicolumn{3}{|c|}{ M. IsOBUtYRATE. $\mathrm{m}=102$} & \multicolumn{3}{|c|}{ Methane. $m=16$} \\
\hline 24 & 754 & .823 & 0 & 1040 & 2.99 \\
\hline 100 & 1122 & 1.19 & 20 & 1201 & 3.33 \\
\hline
\end{tabular}


The quantity $\kappa_{\eta}$ may be interpreted in other ways. $\eta$ represents the rate at which a plane at right angles to a unit velocity gradient loses momentum per square $\mathrm{cm}$. per second. Since $n$ molecules cross the plane per square $\mathrm{cm}$. per second in each direction, $\eta / n$ represents the momentum conveyed by a molecule across the plane on crossing it and recrossing it (sometime) in the opposite direction. According to equation (90) this momentum is equal to $\kappa_{\eta} m_{a} v$ in the case of a gas. Thus $\kappa_{\eta}$ represents the momentum conveyed per unit mass of the molecule at unit volume of a gram molecule of the gas. Hence for substances of equal $\kappa_{\eta}$ and the same molecular concentration the momentum conveyed by a molecule is proportional to its mass.

A molecule crossing the plane gots a distance away from it which on the average is proportional to the volume $v,{ }^{*}$ and the molecule therefore talies $a$ time proportional to $v / V_{a}$ in crossing and recrossing the plane, where $V_{a}$ denotes its average velocity. The amount of momentum conveyed across the plane per second by the same molecule is therefore proportional to $\kappa_{\eta} m_{a} V_{a}$, and thus independent of the volume of the gas.

The quantity $\kappa_{\eta}$, according to equation (91) applied to the gaseous state, is a measurc of the total momentum parallel to the motion of the gas conveyed per square $\mathrm{cm}$. per second across the plane per unit momentum of the momentum of translation motion of a molecule.

A number of formulæ of an empirical nature expressing the variation of $l_{\eta}$, or $\kappa_{\eta} v$, with $T$ at constant $v$, have been given, but which need not concern us here. $\dagger$ It is useful

* This distance is evidently proportional to the chance of the path of the molecule not undergoing a deflection per unit length, which is proportional to $l_{\eta}$, and hence proportional to $v$, since $l_{\eta}=\kappa_{\eta} v$.

$\dagger$ In these investigations the quantity $l_{\eta}$ is supposed to represent molecular free path according to the method of collisions, which is connected with the viscosity by the equation given at the end of this Sub-section. 
to note, however, that $l_{\eta}$ and $\kappa_{\eta}$ are roughly proportional to the square root of the absolute temperature.

The viscosity coefficient of a gas appears to obey approximately the relation of corresponding states, and this holds therefore also for the quantity $\kappa_{\eta}$ according to equation (94). This is shown by Table XI, which gives the ratio

\section{TABLE XI}

\begin{tabular}{|c|c|c|c|c|c|c|}
\hline Substance. & $T_{c}$. & $2 T_{c}$ & $\eta_{c} 10^{7}$. & $\eta_{2 c} 10^{7}$. & $\frac{r_{2 c}}{r_{1} c}$. & $\kappa_{\eta c} 10^{10}$. \\
\hline $\mathrm{CO}_{2}$ & 304.9 & 609.8 & 1625 & 2811 & 1.73 & 2.66 \\
\hline $\mathrm{C}_{2} \mathrm{H}_{4}$ & 282 & 564 & 1022 & 1802 & 1.76 & 2.19 \\
\hline $\mathrm{A}$ & 152 & 304 & 1216 & 2298 & 1.89 & 2.97 \\
\hline $\mathrm{N}_{2} \mathrm{O}$ & 318.4 & 637.8 & 1539 & 2885 & 1.88 & 2.41 \\
\hline $\mathrm{H}_{2}$ & 32 & 64 & 272 & 645 & 2.36 & 6.46 \\
\hline
\end{tabular}

of the viscosities corresponding to the temperatures $T_{c}$ and $2 T_{c}$ for a few substances, where $T_{c}$ denotes the critical temperature, which were interpolated from the viscosity data given in Landolt and Börnstein's Tables, 4th edition. The ratios, it will be seen, are approximately equal to each other. The somewhat large deviation in the case of $\mathrm{H}_{2}$ is probably due to the greater uncertainty attached to the data in that case, and to a lack of sufficiently extensive data for reliable interpolation. The deviation is, however, small in comparison with the difference between the viscosity of $\mathrm{H}_{2}$ and that of each of the other substances. The variation of $\kappa_{\eta}$ with the temperature is therefore approximately expressed by a function of the form $\kappa_{\eta c} \cdot \psi\left(\frac{T}{T_{c}}\right)$, where $\kappa_{\eta}$ c denotes the value of $\kappa_{\eta}$ at the critical temperature, and which is thus a fundamental and characteristic quantity of a gas. Its values for a few substances are given in Table XI, obtained in the same way as the values given in Table $\mathrm{X}$. 
The value of $l_{\eta}$ for a substance in the gaseous state at standard temperature and pressure is obtained by multiplying the corresponding value of $k_{\eta}$ by the volume of a gram molecule of the substance under standard conditions, which according to Avogadro's law has the same value for all substances and is equal to $22,700 \mathrm{cc}$.

It is often of interest to compare the value of $l_{\eta}$ with the average distance of separation $d$ of the molecules, which is given by $d=\left(\frac{m_{a} v}{m}\right)^{1 / 3}=\left(\frac{v}{6.2 \times 10^{23}}\right)^{1 / 3}$, and the ratio of these quantities is therefore given by

$$
\frac{l_{\eta}}{d}=8.5 \times 10^{7} \kappa_{\eta} v^{2 / 3} . \quad . \quad . \quad . \quad .
$$

It will be found that on substituting for $\kappa_{\eta}$ and $v$ at standard temperature and pressure that $l_{\eta}$ is considerably larger than $d$, showing that except for distances of approach of two molecules much less than $d$ the effect of their interaction is small.

If equation (91) is applied to the gaseous state, and $l_{\eta}$ is written for $\kappa_{\eta} v$ according to equation (88) applied to the gaseous state, the equation assumes the form

$$
\eta=\frac{1}{3} \frac{V_{t} m l_{\eta}}{v}=\frac{1}{3} V_{t} \rho l_{\eta}
$$

where $\frac{m}{v}=\rho$ the density of the gas. This equation is usually obtained in treatises on the Kinetic Theory of Gases on the supposition that each molecule has the same velocity $V_{t}$, where $l_{\eta}$ is supposed to denote the mean free path between consecutive collisions of a molecule. Since $l_{\eta}$ cannot represent exactly this quantity the equation has sometimes been modified by the introduction of an appropriate numerical factor as pointed out in Section 33. Another factor is introduced on taking into account the distribution of molec- 
ular velocities since this affects the chance of collision. This represents the most that has been achieved in the way of a Kinetic Theory of substances in connection with viscosity according to the idea of molecular collision.

(b) It will be of interest to consider the values of $l^{\prime}{ }_{\eta}$ of some substances in the liquid state, since they are inferior limits of $l_{\eta}$, from which they differ but little, and they can usually be more easily obtained than the values of $l_{\eta}$. Table XII gives the values of $l^{\prime}{ }_{\eta}$ calculated by means of equa-

TABLE XII

\begin{tabular}{|c|c|c|c|c|}
\hline \multicolumn{5}{|c|}{ ETHER } \\
\hline$t^{\circ} \mathrm{C}$ & $\eta$ & $l^{\prime}{ }_{\eta} 10^{8} \mathrm{~cm}$. & $d 10^{8} \mathrm{~cm}$ & $\frac{l_{\eta}^{\prime}}{d}$. \\
\hline $\begin{array}{l}13.5 \\
25.4 \\
63 \\
78.5 \\
99\end{array}$ & $\begin{array}{l}.001779 \\
.001649 \\
.001338 \\
.001241 \\
.001133\end{array}$ & $\begin{array}{l}2.06 \\
2.08 \\
2.21 \\
2.35 \\
2.91\end{array}$ & $\begin{array}{l}5.49 \\
5.52 \\
5.65 \\
5.70 \\
5.80\end{array}$ & $\begin{array}{l}.376 \\
.377 \\
.392 \\
.412 \\
.502\end{array}$ \\
\hline \multicolumn{5}{|c|}{ Benzene } \\
\hline $\begin{array}{l}15.4 \\
50.1 \\
78.8\end{array}$ & $\begin{array}{l}.004387 \\
.003641 \\
.003000\end{array}$ & $\begin{array}{l}3.25 \\
3.07 \\
3.60\end{array}$ & $\begin{array}{l}5.22 \\
5.29 \\
5.36\end{array}$ & $\begin{array}{l}.623 \\
.581 \\
.486\end{array}$ \\
\hline \multicolumn{5}{|c|}{ Chloroform } \\
\hline $\begin{array}{r}0 \\
20 \\
40 \\
60\end{array}$ & $\begin{array}{l}.003827 \\
.003419 \\
.003073 \\
.002791\end{array}$ & $\begin{array}{l}3.05 \\
2.84 \\
2.73 \\
2.68\end{array}$ & $\begin{array}{l}5.01 \\
5.06 \\
5.10 \\
5.15\end{array}$ & $\begin{array}{l}.607 \\
.562 \\
.535 \\
.520\end{array}$ \\
\hline
\end{tabular}

tion (84) for a number of substances in the liquid state at different temperatures, and for comparison the corresponding values of $d$ the average distance of separation of the mole- 
cules. The value of $A$ used in the equation was not corrected for the distribution of molecular velocities. If corrected according to Maxwell's law the values of $l^{\prime}{ }_{\eta}$ in the Table have to be multiplied by $\sqrt{\frac{3 \pi}{8}}$, or 1.085 . Both the quantities $l^{\prime}{ }_{\eta}$ and $d$ have values of the order of magnitude $10^{-8} \mathrm{~cm}$. The latter values are greater than the former, as is indicated by the values of the ratio $l_{\eta}^{\prime} / d$. The values of $P_{n}$ (which must be reduced to dynes) used in the calculations are contained in Table III, while the values of $\eta$ were calculated by means of the empirical formula of Thorpe and Rodgers.*

Table XIII gives the values of $l_{\eta}^{\prime}$ for $\mathrm{CO}_{2}$ at $40^{\circ} \mathrm{C}$. under high pressures in the gaseous state, and the corresponding values of $d$. According to equation (85), taking the

TABLE XIII

\begin{tabular}{|c|c|c||c|c|c|}
\hline \multicolumn{6}{|c|}{$\mathrm{CO}_{2} \mathrm{AT} 40^{\circ} \mathrm{C}}$. \\
\hline $\begin{array}{c}p \text { in } \\
\text { atmos. }\end{array}$ & $l_{{ }^{\prime}{ }_{\eta} 10^{8} \mathrm{~cm} .}$ & $d 10^{8} \mathrm{~cm}$. & $p$ in atmos. & $l^{\prime}{ }_{\eta} 10^{8} \mathrm{~cm}$. & $d 10^{8} \mathrm{~cm}$. \\
\hline 70 & 6.52 & 7.34 & 94 & 2.84 & 5.16 \\
80 & 4.55 & 6.52 & 100 & 2.83 & 5.03 \\
85 & 3.82 & 5.95 & 112 & 2.81 & 4.90 \\
\hline
\end{tabular}

value of $b$ for $\mathrm{CO}_{2}$ obtained in Section 29, the value of $l_{\eta}^{\prime}$ is smaller than the value of $l_{\eta}$ for the greatest density by about 16 per cent. Thus $l_{\eta}$ and $l_{\eta}^{\prime}$ do not differ much from each other. The values of $P_{n}+p$ and $\eta$ used in these calculations are given by Table XIV.

(c) The number of times $N_{t}$ that the mean momentum transfer distance of a molecule in a substance is passed over per second, given by equation (93), is a quantity of interest. This number for $\mathrm{CO}_{2}$ in the liquid state at $0^{\circ} \mathrm{C}$. under *Phil. Trans., A., 1894, p. 1. 
a pressure of 100 atmos. is $6 \times 10^{12}$, where the value of $V_{t}$ was obtained from Table VIII, $\eta=.000925$, and $\rho=.87$. The number obtained is mainly of interest on account of its great magnitude. It is interesting to note that this number is smaller than the number of times per second the resultant force acting on a molecule due to the surrounding molecules passes through a maximum or minimum.

(d) Let us next apply equation (92) to some of the facts to obtain values of $\Phi_{\eta}$. Table XIV contains values of

TABLE XIV

\begin{tabular}{|c|c|c|c|c|c|}
\hline \multicolumn{7}{c|}{$\mathrm{CO}_{2}$ AT $40^{\circ} \mathrm{C} . \quad \kappa_{\eta}=2.578 \times 10^{-10}$} \\
\hline $\begin{array}{c}p \text { in } \\
\text { atmos. }\end{array}$ & $\begin{array}{c}P_{n}+p \\
\text { in atmos. }\end{array}$ & $\begin{array}{c}v \text { per } \\
\text { grm. mol. }\end{array}$ & $\eta 10^{6}$ & $\Phi_{\eta}$ & $\frac{4.03 \times 10^{3}}{v^{2 \cdot 12}}$ \\
\cline { 2 - 6 } 70 & 128.7 & 245.7 & 200 & $\ldots$. &. .3 \\
80 & 200.9 & 172.2 & 218 & .027 & .073 \\
85 & 295.9 & 130.7 & 269 & .132 & .132 \\
94 & 611.2 & 85.35 & 414 & .292 & .325 \\
100 & 719.3 & 78.96 & 483 & .385 & .385 \\
112 & 854.3 & 73.24 & 571 & .486 & .448 \\
\hline
\end{tabular}

$\Phi_{\eta}$ for $\mathrm{CO}_{2}$ at different pressures at a temperature of $40^{\circ} \mathrm{C}$., at which the gas does not assume the liquid state however great the pressure. The values of $P_{n}+p$ used where calculated by means of Van der Waals' equation of state (Section 26) writing for $b$ the value $42.8 \mathrm{cc}$. per gram molecule, which results from the conditions expressed by equations (57) and (58). The value corresponding to a pressure of 70 atmos. thus obtained is very nearly equal to that given in Table XVI, which was obtained by a different method. The values obtained for $P_{n}+p$ are therefore likely to be at least approximately correct. The values of $\eta$ used are those obtained by Phillips*. Equation (94) gives the value of *Proc. Roy. Soc., A., Vol. LXXXVII, pp. 56-57. 
$\kappa_{\eta}$, for which the data will be found in Table XVI. It will be seen that the values of $\Phi_{\eta}$ are positive, and increase rapidly with the pressure from zero value, which applies when the substance is in the perfectly gaseous state.

This result and previous results furnish information about the effect and importance. of the various factors on which viscosity depends. The positive nature of $\Phi_{\eta}$ indicates according to equations (90), (91), and (92), that molecular interference tends to increase the viscosity of a substance from that it would have otherwise, and according to the Table the effect increases with the density of the substances, as predicted by equation (87). The molecular forces which do not affect the molecular volume tend to increase the total average velocity $V_{\boldsymbol{t}}$ of a molecule from that it would have in the perfectly gaseous state at the same temperature, the effect increasing with the density of the substance (Sections 19 and 29). Therefore according to equation (91) the viscosity of a substance is increased (through the existence of molecular forces which do not affect the molecular volume) corresponding to the increase in $V_{t}$. The existence of the apparent molecular volume $b$, since it is positive, also has the effect of increasing the viscosity, the magnitude of which increases with decrease of volume of the substance according to equation (91). The quantity $b$, it may be noticed, does not affect the total average molecular velocity $V_{t}$ according to its definition (Section 19), but an increase in molecular volume obviously gives rise to an increase in molecular interference. The characteristic quantity $\kappa_{\eta}$ in the viscosity equations is directly connected with the molecular volume, and the molecular forces which do not affect the molecular volume, it increases with an increase in either of these properties. But its value is not affected by molecular interference as will appear on applying equation (88) to the gaseous state which reduces it to the limiting form $\kappa_{\eta}=\left[l_{\delta} / v\right]_{v=\infty}=$ con- 
stant. The foregoing considerations indicate the fundamental reasons for the magnitude of the value of the viscosity of a substance, and the reason why its value increases rapidly with the density when the substance does not behave as a perfect gas.

Interesting information may now also be obtained on the effect of molecular interference on the motion of a molecule.

The positive nature of $\Phi_{\eta}$ indicates, according to equation (89), that the effect of molecular interference is to increase $l_{\eta}^{\prime}$ from the value it would have if it varied proportionally to $v$, which corresponds to the absence of molecular interference. The same remark applies to $l_{\eta}$ which is given by equation (88), since an increase of $b$ would be attended by an increase of molecular interference. This signifies that the chance of momentum being transferred by a migrating molecule to another molecule when moving along the velocity gradient of the substance is reduced by the vicinity of other molecules through the interaction of their forces of attraction and repulsion. But since the excess of momentum of the migrating molecule must eventually be transferred to the medium the act of transference when molecular forces exist is less frequent, but when it occurs more momentum is transferred, than would be the case in the absence of molecular forces. In the former case when a molecule transfers momentum to the medium the difference between its velocity and that of the medium in the direction the medium is moving is greater than in the latter case, and hence we would expect that the amount of momentum transferred would be greater.

Assuming that the series $\Phi_{\eta}$ may approximately be represented by the term $\phi_{\eta} / v^{x}$ the values of $\phi_{\eta}$ and $x$ corresponding to the data in Table XIV were calculated from the values of $\Phi_{\eta}$ corresponding to the pressures 85 and 100 atmos., giving the values $4.03 \times 10^{3}$ and 2.12 respectively. 
The value of $x$ does not differ much from 2 as was predicted. The last column of the Table contains the values of $\phi_{\eta} / v^{x}$ for different pressures, calculated by means of the foregoing values of $\phi_{\eta}$ and $x$. They agree fairly well with the values of $\Phi_{\eta}$ in the preceding column. A better agreement would evidently have been obtained if $\phi_{\eta}$ had been taken a function of $v$ which decreases with increase of $v$.

Table XV gives the values of $\Phi_{\eta}$ at different temperatures for a few liquids (whose density depends of course on the

\section{TABLE XV}

\begin{tabular}{|c|c|c|c|c|}
\hline \multicolumn{5}{|c|}{ Ether } \\
\hline$t^{\circ} \mathrm{C}$ & $\begin{array}{l}\text { Gas } \\
\eta 10^{7}\end{array}$ & $\kappa_{\eta} 10^{10}$. & $\Phi_{\eta}$ & $\phi_{\eta} 10^{4}$ \\
\hline $\begin{array}{l}13.5 \\
99\end{array}$ & $\begin{array}{l}723 \\
942\end{array}$ & $\begin{array}{c}.9612 \\
1.074\end{array}$ & $\begin{array}{l}1.09 \\
1.235\end{array}$ & $\begin{array}{l}1.148 \\
1.857\end{array}$ \\
\hline \multicolumn{5}{|c|}{ ChLoroform } \\
\hline $\begin{array}{r}0 \\
60\end{array}$ & $\begin{array}{r}959 \\
1163\end{array}$ & $\begin{array}{l}1.028 \\
1.129\end{array}$ & $\begin{array}{l}2.783 \\
1.802\end{array}$ & $\begin{array}{l}1.707 \\
2.011\end{array}$ \\
\hline \multicolumn{5}{|c|}{ Benzene } \\
\hline 15.4 & 755 & .974 & 2.772 & 2.158 \\
\hline 78.8 & 1079 & 1.263 & 1.154 & 1.058 \\
\hline
\end{tabular}

temperature), calculated by means of equation (89), using the values of $l_{\eta}^{\prime}$ contained in Table XII. The values of $\kappa_{\eta}$ were calculated by means of equation (94) using the values of $\eta$ corresponding to the gaseous state given in the second column of the Table. It will be seen that the values of $\Phi_{\eta}$ in some cases increase with increase of temperature, 
which, it should be noted, is attended by an increase of the volume, while in the other cases the values decrease. Thus the effect of an increase of the temperature on the value of $\Phi_{\eta}$ appears to be in the opposite direction to the effect of an inerease of the volume. If the quantity $\Phi_{\eta}$ behaves similarly for all substances, as we might expect, the foregoing results would indicate that at constant temperature it would decrease with increase of volume, as the preceding results have already shown, and increase with increase of temperature at constant volume. This indicates that the chance of transfer of momentum by a migrating molecule to a molecule of the surrounding medium through the existence of molecular forces is decreased by an increase of temperature.

Table XV also contains the values of $\phi_{\eta}$ in the approximate expression of $\phi_{\eta} / v^{x}$ for $\Phi_{\eta}$, putting $x=2$. The values of $\eta$ for different volumes at constant temperature of the substances mentioned in the Table might now be approximately calculated by means of equation (92) on calculating the values of $P_{n}+p$ by means of an empirical equation of state according to Section 21 .

Further investigation in connection with the experimental values of $\eta$ of liquids must proceed mainly along the line of comparing the values of the characteristic quantity $\Phi$ of different substances at various temperatures and volumes. This might furnish some information as to how this quantity depends on the molecular weight besides on the volume and temperature, and incidentally furnish further information in connection with the molecular forces.

(e) It is interesting to apply equation (91) to a substance not in the perfectly gaseous state at volumes for which $b$ is small in comparison with $v$, and $\Phi_{\eta}$ small in comparison with unity, in which case the equation becomes

$$
\eta=\frac{1}{3} V_{t} m_{\kappa_{\eta}}
$$


Corresponding to the smallest value of $v$ for which these conditions hold $\eta$ usually differs considerably from that applying to the substance in the perfectly gaseous state, and this therefore also holds for $V_{t}$ and $n$. The value of $V_{t}$ may therefore be calculated with fair accuracy by means of the foregoing equation over a region at the beginning of which a substance begins to deviate from the gas laws. The corresponding values of $P_{n}+p$, and hence of $P_{n}$, may be obtained from equation (92). Table XVI contains a

\section{TABLE XVI}

\begin{tabular}{|c|c|c|c|c|c|}
\hline \multicolumn{7}{|c|}{$\mathrm{CO}_{2}$ AT $40^{\circ} \mathrm{C} . \quad \kappa_{\eta}=2.578 \times 1.085 \times 10^{-10}$} \\
\hline $\begin{array}{c}p \text { in } \\
\text { atmos. }\end{array}$ & $\eta \times 10^{6}$. & $\begin{array}{c}v \text { per } \\
\text { grm. mol. }\end{array}$ & $\begin{array}{c}P_{n}+p \\
\text { in atmos. }\end{array}$ & $\begin{array}{c}P_{n} \text { in } \\
\text { atmos. }\end{array}$ & $V_{t} / V_{a}$. \\
\hline 1 & 157 & 25,550 & 1 & $\ldots .6$ & 1.000 \\
40 & 176 & 589.8 & 48.59 & 8.6 & 1.121 \\
60 & 187 & 328.7 & 92.70 & 32.7 & 1.191 \\
70 & 200 & 245.7 & 132.5 & 62.5 & 1.274 \\
\hline
\end{tabular}

set of calculations carried out for $\mathrm{CO}_{2}$ at $40^{\circ} \mathrm{C}$. over a range of volumes for which the foregoing conditions hold, since approximately $b=12$, Section 29 , and $\Phi_{\eta}=\frac{4.03 \times 10^{3}}{v^{2.12}}$ according to Table XIV. The value of $\kappa_{\eta}$ in equation (96) was determined by applying equation (94) to $\mathrm{CO}_{2}$ in the gaseous state at a pressure of one atmos. and temperature $40^{\circ} \mathrm{C}$., and correcting the value obtained according to Maxwell's law. It will be seen that $P_{n}$ and the ratio $V_{t} / V_{a}$, where $V_{a}$ denotes the average velocity of translation of a molecule of $\mathrm{CO}_{2}$ in the gaseous state, which is given by equation (8) and the equation $V_{a}=.922 \mathrm{~V}$, gradually increase with increase of pressure. Thus the more the molecules come under each other's influence the greater the total average 
velocity above that in the gaseous state, which falls into line with what has been obtained before. It is of importance to notice, however, that the foregoing deduction of the result does not depend on the results of Sections 16 and 17 .

(f) On using the term $\phi_{\eta} / v^{2}$ for the series $\Phi_{\eta}$ in equation (90), which holds approximately according to Sub-section $d$, and substituting for $n$ the expression given by equation (66) retaining only the first two terms, we obtain the equation

$$
\eta=\left(\frac{C_{1}}{v}+\frac{C_{2}}{v^{2}}\right)\left(\frac{v^{2}+\phi_{\eta}}{v}\right) \frac{m_{a} \kappa_{\eta}}{v-b}
$$

This equation contains the three unknowns $b, C_{2}$, and $\phi_{\eta}$. They may be determined by applying the equation at constant temperature to a substance at three densities not differing much from each other. The values of $n$ and $V_{t}$ may then at once be obtained similarly as in Section 29 .

It may be noted, however, that it is preferable to determine the quantities $b, C_{2}$, and $\phi_{\eta}$, if possible, without using simultaneous equations, or using as few as possible, as this gives more reliable results. For the variables of a set of simultaneous equations may usually be varied over a considerable range and yet approximately satisfy the equations, and hence slight errors in the constants of the equations (furnished by experiment) may considerably affect the values obtained for the variables.

(g) In Sub-section (a) of this Section it was shown that the values of $\eta$ for substances in the gaseous state obey the relation of corresponding states. This is also found to hold when the substances are not in the gaseous state,* as is shown by the approximate constancy of the ratio of $\eta_{1}$ to $\eta_{2}$ corresponding to the temperatures $T_{c} / 2$ and $4 T_{c} / 7$, shown by Table XVII for a number of liquids. Since the quantities $P_{\eta}+p, v, T$, and $\kappa_{\eta}$ in equation (92) obey this

* R. D. Kleeman, Proc. Camb. Phil. Soc., Vol. XVI, Pt. 7, p. 633. 


\section{TABLE XVII}

\begin{tabular}{|c|c|c|c|c|c|}
\hline \multicolumn{3}{|c|}{ ETHELENE-BROMIdE $\left(\mathrm{C}_{4} \mathrm{H}_{4} \mathrm{Br}_{2}\right)$} & \multicolumn{3}{|c|}{ Ethelene ChLORIDE $\left(\mathrm{C}_{4} \mathrm{H}_{4} \mathrm{Cl}_{2}\right)$} \\
\hline$t^{\circ} \mathrm{C}$ & $\eta_{1}$ and $\eta_{2}$. & $\eta_{1} / \eta_{2}$ & $t^{\circ} \mathrm{C}$ & $\eta_{1}$ and $\eta_{2}$. & $\eta_{1} / \eta_{2}$ \\
\hline $\begin{array}{l}18.4 \\
60\end{array}$ & $\begin{array}{l}.01767 \\
.009985\end{array}$ & 1.770 & $\begin{array}{r}7.7 \\
47.8\end{array}$ & $\begin{array}{l}.01000 \\
.005903\end{array}$ & 1.693 \\
\hline \multicolumn{3}{|c|}{ Ethyl propionate $\left(\mathrm{C}_{5} \mathrm{H}_{10} \mathrm{O}_{2}\right)$} & \multicolumn{3}{|c|}{ Butyric ACID $\left(\mathrm{C}_{4} \mathrm{H}_{8} \mathrm{O}_{2}\right)$} \\
\hline $\begin{array}{l}-3 \\
38.6\end{array}$ & $\begin{array}{l}.007040 \\
.003864\end{array}$ & 1.822 & $\begin{array}{l}32.5 \\
76.1\end{array}$ & $\begin{array}{l}.01255 \\
.007016\end{array}$ & 1.777 \\
\hline \multicolumn{3}{|c|}{ ThIophen $\left(\mathrm{C}_{4} \mathrm{H}_{4} \mathrm{~S}\right)$} & \multicolumn{3}{|c|}{ Benzene $\left(\mathrm{C}_{6} \mathrm{H}_{6}\right)$} \\
\hline $\begin{array}{l}22.1 \\
64.3\end{array}$ & $\begin{array}{l}.00644 \\
.00408\end{array}$ & 1.579 & $\begin{array}{r}3.8 \\
43.3\end{array}$ & $\begin{array}{l}.008680 \\
.004740\end{array}$ & 1.831 \\
\hline \multicolumn{3}{|c|}{ ETHYL BENZENE $\left(\mathrm{C}_{8} \mathrm{H}_{10}\right)$} & \multicolumn{3}{|c|}{ Propionic acid $\left(\mathrm{C}_{3} \mathrm{H}_{6} \mathrm{O}_{2}\right)$} \\
\hline $\begin{array}{l}36.7 \\
80.9\end{array}$ & $\begin{array}{l}.00550 \\
.00357\end{array}$ & 1.540 & $\begin{array}{l}33.4 \\
77.2\end{array}$ & $\begin{array}{l}.009169 \\
.005605\end{array}$ & 1.635 \\
\hline \multicolumn{3}{|c|}{ Octane $\left(\mathrm{C}_{8} \mathrm{H}_{18}\right)$} & \multicolumn{3}{|c|}{ CARBON TETRACHLORIDE $\left(\mathrm{CCl}_{4}\right)$} \\
\hline $\begin{array}{l}11.6 \\
52.2\end{array}$ & $\begin{array}{l}.006060 \\
.003783\end{array}$ & 1.603 & $\begin{array}{c}5 \\
44.7\end{array}$ & $\begin{array}{l}.0124 \\
.00696\end{array}$ & 1.781 \\
\hline
\end{tabular}

relation (Section 28 and Sub-section (a) of this Section), it follows from this equation that the factor $1+\Phi_{\eta}$ also approximately obeys the relation of corresponding states. We may therefore write $1+\Phi_{\eta}=\psi\left(\frac{T_{e}}{T}, \frac{\rho_{c}}{\dot{\rho}}\right)$, where $T_{c}$ denotes the critical temperature, and $\rho_{c}$ the critical density of the substance. 


\section{Formula for the Viscosity of Mixtures.}

It will be evident from an examination of the investigation in the previous Section that the effect of each molecule of a substance on its viscosity is additive in character. Therefore in the case of a mixture the effect of the different sets of molecules is additive. It will easily be seen therefore that in the case of a mixture of molecules $e$ and $r$ the viscosity is given by

$$
\eta=n_{r} m_{a r} l_{\eta r}+{ }_{r} n_{e} m_{a e} l_{\eta e}, \ldots . . . .
$$

where $l_{\eta^{r}}$ denotes the mean momentum transfer distance of a molecule $r$ of absolute mass $m_{a r}, n_{r}$ the number of molecules $r$ crossing a square $\mathrm{cm}$. from one side to the other per second, and the remaining symbols have similar meanings with respect to the molecules $e$. For $l_{\eta r}$ and $l_{\eta e}$ we may write

$$
l_{\eta r}=\kappa_{\eta r}^{\prime} \frac{v_{r}}{v_{r}-b_{r}^{\prime}} \frac{N}{N_{e r}}\left(1+\Phi_{\eta r}^{\prime}\right), \ldots . .
$$

and

$$
l_{\eta e}=\kappa^{\prime}{ }_{\eta e} \frac{v_{e}}{v_{e}-b^{\prime}} \frac{N}{N_{e r}}\left(1+\Phi^{\prime}{ }_{\eta e}\right),
$$

similarly as in the previous Section, where $v_{r}$ denotes the volume of the mixture containing a gram molecule of molecules $r, b_{r}^{\prime}$ the apparent volume of the molecules $r$ and $e$ in the volume $v_{r}$ with respect to the motion of a molecule $r$ (Section 20), $\kappa^{\prime}{ }_{\eta r}$ a characteristic constant of the molecules $r$ when the mixture is in the gaseous state, $N_{e r}$ the total concentration of the molecules $e$ and $r, N$ the number of molecules in a gram molecule of a pure substance, $\Phi_{\eta r}$ the effect of the molecules of the mixture on a molecule $r$ interacting with a molecule $r$ or $e$, or the interference function of the mixture with respect to the molecules $r$, and the remaining quantities have similar meanings with respect to 
the molecules $e$. It will be recognized that in deducing the foregoing two equations along similar lines as equation (88), we have written

$$
l_{\eta r}^{\prime}=\kappa^{\prime}{ }_{\eta r} \frac{N}{N_{e r}}\left(1+\Phi_{\eta r}, \text { and } l_{\eta e}^{\prime}=\kappa^{\prime}{ }_{\eta e} \frac{N}{N_{e r}} 1+\Phi_{\eta c}\right),
$$

or replaced volume by concentration, which in the case of mixtures is more convenient. It should be noted that in the case of a pure substance-say $e$, we have $v=\frac{N}{N_{e}}$. It is also more convenient to regard $\Phi^{\prime}{ }_{\eta e}$ and $\Phi^{\prime}{ }_{\eta r}$ as a series of powers of the concentration instead of the volume. As a first approximation we may write

$$
\Phi_{\eta r}^{\prime}=\phi_{\eta r}^{\prime} \frac{N^{2}{ }_{e r}}{N^{2}} \quad \text { and } \quad \Phi_{\eta e}^{\prime}=\phi_{\eta e}^{\prime} \frac{N^{2}{ }_{e r}}{N^{2}}
$$

similarly as in the previous Section. It will be easy to see that the various quantities involved are functions of the ratio $\xi$ of the number of molecules $r$ to $e$, as well as of their nature.

On applying equation (98) to the gaseous state after substituting from equations (99) and (100), it becomes

$$
\eta=\left\{\frac{\xi}{\xi+1} \kappa^{\prime}{ }_{\eta r} \sqrt{m_{r}}+\frac{1}{1+\xi} \kappa^{\prime}{ }_{\eta e} \sqrt{m_{e}}\right\} \sqrt{\frac{R T}{3}},
$$

by the help of equation (21), and since

$$
\xi=\frac{v_{e}}{v_{r}}, \text { and } \quad \frac{N}{v_{e}}+\frac{N}{v_{r}}=N_{e r},
$$

where $N$ denotes the number of molecules in a gram molecule, and $m_{r}$ and $m_{e}$ the molecular weights of a 
molecule $r$ and $e$ respectively in terms of that of the hydrogen atom. This equation expresses a relation between the quantities $\kappa^{\prime}{ }_{\eta r}$ and $\kappa^{\prime}{ }_{\eta e}$, which is of use in determining them.

Approximate values of $\kappa^{\prime}{ }_{\eta r}$ and $\kappa^{\prime}{ }_{\eta e}$ may be obtained from the values of $\kappa_{\eta r}$ and $\kappa_{\eta e}$ corresponding to the constituents separated from each other and in the gaseous state. In the case of a pure substance $r$ in the gaseous state $l_{\eta r}=\kappa_{\eta r} v_{r}=\kappa_{\eta r} \frac{N}{N_{r}}$. Thus $l_{\eta r}$ varies inversely as the probability of a molecule $r$ coming under the influence of another molecule $r$ under given conditions, which is proportional to $N_{r}$, and hence $1 / \kappa_{\eta r}$ is the probability factor of $N_{r}$. In the case of a mixture of molecules $r$ and $e$ in the gaseous state the transfer distance $l_{\eta r}$ varies inversely as the probability of a molecule $r$ coming under the influence of a molecule $r$ or $e$. This probability consists of the sum of the probabilities of a molecule $r$ coming under the influence of another molecule $r$, and of coming under the influence of a molecule $e$, since these processes are independent. Now the first probability is equal to $\frac{N_{r}}{N} \frac{1}{\kappa_{\eta r}}$, where $\kappa_{\eta r}$ refers to the molecules $r$ in the pure state, since the two probabilities are independent and we may therefore suppose the molecules $e$ absent. The second probability is equal to $\frac{N_{e}}{N} \frac{1}{\kappa_{x}}$, where $\frac{1}{\kappa_{x}}$ is the probability factor in this case. Hence we have

$$
\frac{1}{l_{\eta r}}=\frac{N_{e r}}{N} \frac{1}{\kappa_{\eta r}^{\prime}}=\frac{N_{r}}{N} \frac{1}{\kappa_{\eta r}}+\frac{N_{e}}{N} \frac{1}{\kappa_{x}},
$$

or

$$
\frac{1}{\kappa_{\eta r}^{\prime}}=\left\{\frac{N_{r}}{\kappa_{\eta r}}+\frac{N_{e}}{\kappa_{x}}\right\} \frac{1}{N_{e r}} . \ldots . . .
$$


The factor $1 / \kappa_{x}$ may be expressed as a mean of the factors $1 / \kappa_{\eta r}$ and $1 / \kappa_{\eta}$ referring to the substances $r$ and $e$ in the pure state. Thus we may write

$$
\frac{1}{\kappa_{x}}=\frac{1}{4}\left\{\frac{1}{\kappa_{\eta r^{1 / 2}}}+\frac{1}{\kappa_{\eta e^{1 / 2}}}\right\}^{2},
$$

or we may write

$$
\frac{1}{\kappa_{x}}=\frac{1}{2}\left\{\frac{1}{\kappa_{\eta r}}+\frac{1}{\kappa_{\eta}}\right\},
$$

which would hold approximately. Thus an approximate value of $\kappa_{\eta \tau}^{\prime}$ could be obtained from equation (102) in terms of quantities referring to the constituents of the gaseous mixture in the pure state. Similarly an approximate expression for $\kappa_{\eta e}^{\prime}$ may be obtained. The accuracy of the values obtained may be checked by means of equation (101).

Approximate values of $\phi_{\eta r}^{\prime}$ and $\phi_{\eta}^{\prime}$ may be calculated from the values of $\phi_{\eta r}$ and $\phi_{\eta e}$ referring to the isolated constituents of the mixture. In the case of a pure substance $r$ the term $\phi_{\eta r} N_{r}^{2} / N^{2}$ is a measure of the probability of a molecule $r$ coming under the influence of another molecule $r$ and the pair coming under the influence of a third molecule $r$. In the case of a mixture of molecules $r$ and $e$ the term $\phi^{\prime}{ }_{\eta r} N^{2}{ }_{e r} / N^{2}$ is correspondingly a measure of the probability of a molecule $r$ coming under the influence of another molecule $r$ and the pair coming under the influence of a molecule $r$ or $e$, or a molecule $r$ coming under the influence of a molecule $e$ and the pair coming under the influence of a molecule $r$ or $e$. These four probabilities may be taken as approximately independent of each other, and the total probability therefore equal to their sum. We may therefore write

$$
\phi_{\eta r}^{\prime} \frac{N^{2}{ }_{r}{ }^{2}{ }_{e}}{e_{e}}=\phi_{\eta r} \frac{N^{2} r}{N^{2}}+\phi_{x} \frac{N_{r} N_{e}}{N^{2}}+\phi_{\nu} \frac{N_{r} N_{e}}{N^{2}}+\phi_{z} \frac{N^{2} e_{e}}{N^{2}},
$$


where $\phi_{\eta r}$ refers to molecules $r$ in the pure state, and $\phi_{x}$, $\phi_{\nu}$, and $\phi_{z}$, are appropriate probability factors. These factors may approximately be expressed in terms of quantities referring to the molecules $r$ and $e$ in the pure state. Thus we may write

$$
\begin{aligned}
& \phi_{x}=\left(\phi_{\eta_{r}}^{2} \phi_{\eta e}\right)^{1 / 3}, \\
& \phi_{\nu}=\left(\phi_{{ }_{r}{ }^{r}} \phi_{\eta e}\right)^{1 / 3}, \\
& \phi_{z}=\left(\phi_{\eta \tau} \phi^{2}{ }_{\eta e}\right)^{1 / 3},
\end{aligned}
$$

approximately; or we may write

$$
\begin{aligned}
& \phi_{x}=\frac{1}{3}\left(2 \phi_{\eta r}+\phi_{\eta e}\right), \\
& \phi_{\nu}=\frac{1}{3}\left(2 \phi_{\eta r}+\phi_{\eta e}\right), \\
& \phi_{z}=\frac{1}{3}\left(\phi_{\eta r}+2 \phi_{\eta e}\right),
\end{aligned}
$$

approximately. Similarly an approximate expression for $c^{\prime}{ }_{n e}$ may be obtained.

The quantities just considered may be determined directly by the following method. In conformity with equation (102) we may write

$$
\frac{1}{\kappa_{\eta r}{ }^{\prime}}=\frac{1}{N_{e r}}\left\{N_{r} a_{r}+N_{e} a_{r e}\right\},
$$

and

$$
\frac{1}{\kappa_{\eta \ell}^{\prime}}=\frac{1}{N_{e r}}\left\{N_{e} a_{e}+N_{r} a_{r e}\right\}
$$

On substituting for $\kappa^{\prime}{ }_{\eta r}$ and $\kappa^{\prime}{ }_{\eta e}$ from these equations in equation (101) and applying it to a gaseous mixture at three different relative concentrations at constant temperature three simultaneous equations are ubtained from which the constants $a_{r}, a_{r e}$, and $a_{e}$ may be determined. 
Similarly in conformity with equation (103) we may write

$$
\phi_{\eta_{r}}^{\prime}=\frac{1}{N^{2}{ }_{e r}}\left\{b_{r} N^{2}{ }_{r}+\dot{b}_{r r e} N_{e} N_{r}+b_{r e e} N^{2}{ }_{e}\right\}
$$

and

$$
\phi^{\prime}{ }_{\eta e}=\frac{1}{N^{2}{ }_{e r}}\left\{b_{e} N^{2}{ }_{e}+b_{r e e} N_{e} N_{r}+b_{r r e} N_{r}^{2}\right\} .
$$

On substituting for $\phi_{\eta r}^{\prime}$ and $\phi_{\eta e}^{\prime}$ in equation (98) transformed by means of subsequent equations as indicated, and applying it to four different relative concentrations of the mixture at constant temperature four simultaneous equations will be obtained from which the constants $b_{r}, b_{r r e}, b_{r e e}$, and $b_{e}$, may be determined. The values of $b_{r}^{\prime}$ and $b^{\prime}{ }_{e}$ involved, it may be pointed out, may be obtained by the help of Section 29.

Knowing the values of the foregoing seven constants and the values of $b_{e}^{\prime}$ and $b_{r}^{\prime}$, the viscosity may be calculated for any density and relative concentration of the constituents.

Similarly a mixture of more constituents than two may be treated.

\section{The Coefficient of Conduction of Heat.}

When heat flows from one part of a substance to another at a different temperature without a bodily transference of matter taking place the heat is said to be propagated by conduction. The heat energy is then transferred from one molecule to another in the direction of the flow of heat through their interaction by means of their forces of attraction and repulsion. The coefficient of conduction of heat is usually defined in connection with the flow of heat across a slab of material of thickness $d$ and infinite extent whose surfaces are kept at the different temperatures $t_{1}$ and $t_{2}$, 
where $t_{1}>t_{2}$ say. The quantity of heat $Q$ transferred per second per square $\mathrm{cm}$. of each surface is defined by

$$
Q=\left(\frac{t_{1}-t_{2}}{d}\right) C, \quad \cdot \quad \cdot \quad \cdot \quad .
$$

where $C$ is a constant which is called the coefficient of conduction of heat, and $\frac{t_{1}-t_{2}}{d}$ is called the temperature gradient of the flow of heat. When the gradient is equal to unity it follows from the equation that the coefficient $C$ is equal to the quantity of heat transferred from one side of the slab to the other per second per square cm. of surface. The lines of flow of heat in the foregoing arrangement would obviously be everywhere perpendicular to each surface of the slab. In practice with a slab of finite dimensions this is realized only near the central portion, and the amount of heat transferred is therefore measured for this portion only, the rest of the slab acting as a guard ring arrangement.

As in the case of viscosity, the flow of heat in a substance is directly connected with the nature of the motion of a molecule, and it will therefore be necessary to define a quantity connected with the path described by a molecule in migrating from one portion of the substance to another.

\section{The Mean Heat Transfer Distance of a} Molecule in a Substance.

Consider a substance which is at a higher temperature in the plane $A B$, Fig. 14, than in the parallel plane $C D$, and which has a uniform temperature gradient between the planes. Let $a b c$ denote the path of a molecule migrating progressively into layers at lower temperatures. The molecule loses heat energy in continually varying amounts to the medium at the expense of its kinetic energy (Section $16)$, and the change in its potential energy of attraction 
brought about by passing progressively into denser layers of the substance. We may suppose that the medium acquires and loses energy at certain points only near the path of the migrating molecule, which energy is abstracted from, or transferred to, the migrating molecule as the case may be. The amount of energy lost at a point will be taken equal to $T_{2} S_{m}$, where $T_{2}$ denotes the absolute temperature at the point, and $S_{m}$ the internal specific heat at constant pressure of the molecule, while the energy acquired at the point will be taken equal to $T_{1} S_{m}$, where $T_{1}$ denotes the absolute temperature at the preceding point. The internal specific heat $S_{m}$ of a molecule is equal to the internal specific heat

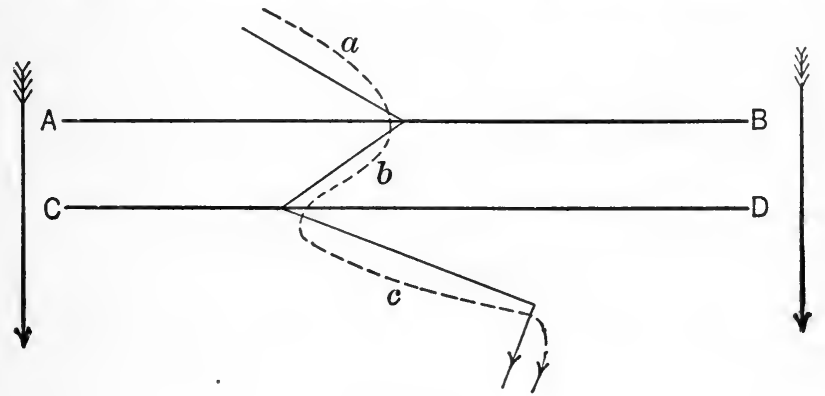

FIG. 14.

at constant pressure of a gram of molecules (Section 13) divided by the number of molecules it contains. The line joining two consecutive points will be called a heat transfer distance in the substance, since the molecule abstracts the quantity of heat $T_{1} S_{m}$ at one extremity of the distance and transfers it to the other extremity, while at the latter point it abstracts the quantity of heat $T_{2} S_{m}$ and transfers it to the other extremity of the adjacent distance, etc. Let us suppose, similarly as in the case of viscosity, that the positions of the points associated with the path of a migrating molecule are so selected that the number of heat transfer 
distances, and the number of molecular paths, cutting a plane of one square $\mathrm{cm}$., are equal to one another, and that all directions of a transfer distance in space are equally probable. These two conditions determine the positions and lengths of the transfer distances. These distances are not equal to each other, but are grouped about a mean distance $l_{c}$ according to the distribution law of Clausius given in Section 31.

The mean heat transfer distance of a molecule is evidently not equal to the mean momentum transfer distance in 'viscosity, for one refers to the transfer of momentum and the other to energy under different conditions. Experimental evidence will be considered later showing that that is so. In treatises on the Kinetic Theory of Gases both distances are usually assumed to be equal to the average distance between two consecutive collisions of a molecule.

We will now deduce formulæ involving the mean heat transfer distance as defined in this Section.

38. Formula for the Coefficient of Conduction of Heat in Terms of Other Quantities.

Let us suppose that the temperature of a substance is higher in the plane $A B$, Fig. 15, than in the parallel plane $C D$. Suppose that $n$ molecules cross the plane $E F$ from one side to the other. This migration of molecules gives rise to a transference of heat across the plane from top to bottom which per square $\mathrm{cm}$. is numerically equal to the coefficient of conduction of heat if the temperature gradient is unity. This amount of heat may be expressed in terms of other quantities as follows. Consider the heat transfer distances which cut the plane $E F$ and are inclined at an angle $\theta$ to a perpendicular to the plane. For purposes of calculation we may suppose that the upper extremities of these distances are located at the same point which may be 
taken to lie in the plane $E F$. It.follows then from the figure, which shows a section of a hemisphere of radius $z$ having the point mentioned as center, that the number of these distances is equal to

$$
n \frac{2 \pi r z \cdot d \theta}{2 \pi z^{2}}
$$

where $z$ denotes the length of one of these distances, $r=$ $z \sin \theta$, and the number is thus equal to $n \sin \theta \cdot d \theta$.

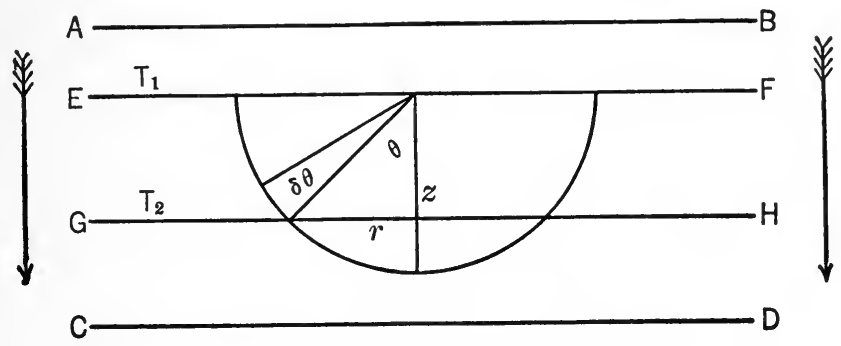

FIG. 15.

If $n_{z}$ denote the number of the foregoin $r$ distances whose lengths lie between $z$ and $z+d z$, we have

$$
n_{z}=n \sin \theta \cdot d \theta \cdot \frac{z}{l_{c}^{2}} e^{-\frac{2}{l_{c}}} \cdot d z
$$

by the help of equation (81). Each of the molecules corresponding to the foregoing distances abstract the energy $T_{1} S_{m}$ from the plane $E F$ which is at the temperature $T_{1}$, and transfers it to the plane $G H$ which is at the temperature $T_{2}$. An equal number of molecules inclined at the angle $\theta$ to a line at right angles to the plane $E F$ move in the opposite direction. Each of these molecules abstracts the energy $T_{2} S_{m}$ from the plane $G H$ and transfers it to the plane $E F$. Thus on the whole these two sets of molecules transfer the 
energy $n_{z}\left(T_{1} S_{m}-T_{2} S_{m}\right)$ across the plane $E F$. On taking the temperature gradient equal to unity we have

$$
\frac{T_{1}-T_{2}}{z \cos \theta}=1,
$$

and the preceding expression for the energy trainsfer becomes

$$
n_{z} S_{m} z \cos \theta .
$$

The total energy transferred per square $\mathrm{cm}$. across the plane $E F$ is obtained on substituting for $n_{2}$ in the foregoing expression and integrating it from 0 to $\infty$ with respect to the transfer distance $z$, and from 0 to $\pi / 2$ with respect to the angle $\theta$, which gives

$$
C=n S_{m} \int_{0}^{\frac{\pi}{2}} \int_{0}^{\infty} \cos \theta \sin \theta \frac{z^{2}}{l_{c}^{2}} e^{-\frac{z}{l_{c}}} \cdot d \theta \cdot d z,
$$

where $C$ denotes the coefficient of conduction of heat. The integral in this equation can be shown to be equal to $l_{0}$ (Section 34), which reduces the equation to

$$
C=n S_{m} l_{c}, \quad \text {. . . . . . }
$$

where $n$ denotes the number of molecules crossing a square $\mathrm{cm}$. from one side to the other, $l_{c}$ the mean heat transfer distance, and $S_{m}$ the internal specific heat at constant pressure per molecule. If $S_{\diamond}$ denote the internal specific heat per gram at constant pressure of the substance we have

$$
S_{m}=S_{\imath} m_{a}, \quad . \quad \text {. . . . . }
$$

where $m_{a}$ denotes the absolute molecular weight of a molecule, and the preceding equation may be written

$$
C=n m_{a} S_{0} l_{c} .
$$

By means of this equation the value of $l_{c}$ for any state of 
matter may be calculated, since $C$ and $S_{g}$ may be measured directly and $n$ determined indirectly according to Section 29 .

The quantity $l_{c}$ may be expressed in terms of other quantities similarly as the quantity $l_{\eta}$ in Section 34 , by representing the substance by another substance possessing the same coefficient of conduction of heat, internal specific heat at constant pressure, and expansion pressure, but whose molecules possess no apparent volume $b$. The coefficient of conduction of the representative substance is evidently given by

$$
C=n^{\prime} m_{a} S_{g} l_{c}^{\prime}
$$

where $n^{\prime}$ and $l^{\prime}{ }_{c}$ referring to the representative substance have meanings similar to $n$ and $l_{c}$ in equation (107). Since the representative substance has the same expansion pressure as the original substance, and the molecules of the former substance have no volume, we have according to equations (38) and (45) that

$$
n^{\prime}=n \frac{v}{v-b}=\frac{P_{n}+p}{A / 2}
$$

where the quantities $n, b, P_{n}, p, v$, and $A$ refer to the original substance. The preceding equation may therefore be written

$$
C=\frac{v}{v-b} n m_{a} S_{g} l_{c}^{\prime}=\frac{2\left(P_{n}+p\right)}{A} m_{a} S_{g} l_{c}^{\prime} . .
$$

This equation determines $l_{c}^{\prime}$ since each of the other quantities it contains may be determined from the original substance.

The relation between $l_{c}$ and $l_{c}^{\prime}$ according to equations (107) and (108) is given by

$$
l_{c}=l_{c}^{\prime} \frac{v}{v-b}, \quad \cdot \quad \cdot \quad \cdot \quad \cdot
$$

and thus $l_{c}^{\prime}$ is an inferior limit of $l_{c}$. 
Equation (108) may be given another form along the same lines as equation (84) in Section 34. If the molecules in the representative substance were devoid of molecular forces which extend beyond a small distance from each molecule, the quantity $l_{c}$ would vary inversely as the chance of one molecule meeting another, or be proportional to the volume $v$, and we may write

$$
l_{c}^{\prime}=\kappa_{c} v,
$$

where $\kappa_{c}$ is a constant at constant temperature. The existence of molecular forces extending some distance from each molecule gives rise to the interaction of two molecules not being independent of the surrounding molecules, and hence $l_{c}^{\prime}$ not being proportional to $v$. This effect may be expressed by a factor introduced into the right-hand side of the foregoing equation, which is obtained in the same way as a similar factor in Section 34. Since the chance of a molecule interacting with another molecule and the pair coming under the influence of $r$ molecules, is proportional to $1 / v^{r+1}$, the factor in question may be written

$$
1+\frac{\phi_{c}^{\prime}}{v^{2}}+\frac{\phi^{\prime \prime}{ }_{c}}{v^{3}}+=1+\Phi_{c}
$$

The quantities $\phi_{c}^{\prime}, \phi_{c}^{\prime \prime}, \ldots$, which are functions of ${ }^{\prime} T$ and $v$, insensitive to $v$, are not identical, it should be noted, with the quantities $\phi_{\eta}^{\prime}, \phi^{\prime \prime}{ }_{\eta}, \ldots$, in Section 34, because the quantities $l_{\eta}$ and $l_{c}$ have somewhat different meanings, but they will probably not differ much from each other. The quantities $l_{c}$ and $l_{c}^{\prime}$ are therefore given by the equations

$$
l_{c}=\frac{v}{v-b} l_{c}^{\prime}=\frac{\kappa_{c} v^{2}}{v-b}\left(1+\Phi_{c}\right), \ldots . .
$$

and

$$
l_{c}^{\prime}=\kappa_{c} v\left(1+\Phi_{c}\right) . . . . . .
$$


Equation (111) may be interpreted similarly as equation (88). The interaction of two molecules is modified by the surrounding molecules, an effect which was called molecular interference. The value of the apparent molecular volume $b$ in the equation depends to some extent (which is probably small) on molecular interference, while $\Phi_{c}$ depends wholly on it. The latter quantity may therefore be called the interference function in heat conduction. In value it probably differs not much from that of the interference function $\Phi_{\eta}$ in viscosity. Therefore according to equation (111) the effect of molecular interference on $l_{c}$ which is not represented by $b$ (a quantity which also occurs in equation (88)), is represented by the function $\Phi_{c}$, which may now be used without referring to the representative substance.

Equation (107) may therefore be written in the forms

$$
\begin{aligned}
& C=\frac{v^{2}}{v-b} n m_{a} \kappa_{c} S_{g}\left(1+\Phi_{c}\right), \ldots . \\
& C=\frac{v}{3(v-b)} V_{t} m_{\kappa_{c}} S_{g}\left(1+\Phi_{c}\right), \ldots
\end{aligned}
$$

and

$$
C=\frac{2}{A}\left(P_{n}+p\right) v m_{a \kappa_{c}} S_{l}\left(1+\Phi_{c}\right), \quad . \quad .
$$

by the help of equations (35) and (46), and remembering that $N_{c} m_{a} v=m$. The values of $n, b$, and $V_{t}$, the number of molecules crossing a square $\mathrm{cm}$., the apparent molecular volume in cubic cms. per gram molecule, and the total average velocity in cms. respectively, may be determined by the method described in Section 29. The value of the intrinsic pressure $P_{n}$, which must be expressed in dynes similarly as the pressure $p$, may be determined by the method described in Section 21. The internal specific heat $S_{g}$ per gram at constant pressure, and the conduction 
of heat $C$ per second across a square $\mathrm{cm}$. at right angles to unit heat gradient, must be expressed in terms of the same heat units, which for convenience may be taken the calorie, or the meehanical heat unit the erg. The ealculated values of $n, b$, and $V_{\imath}$ depend on the distribution of velocities assumed for the molecules; they may be corrected according to Maxwell's law, if desired, in the way described. The value of $A$ in equation (115) not corrected for the distribution of molecular velocities is given by

$$
A=5.087 \times 10^{-20} \sqrt{T m}
$$

according to Section 11. If corrected according to Maxwell's law the value has to be multiplied by $\sqrt{\frac{3 \pi}{8}}$, or 1.085 . It will appear, however, from Sub-section $a$ that the calculated value of $\kappa_{c}$ similarly corrected involves the factor 1.085 and that therefore the uncorrected values of $A$ and $\kappa_{c}$ may be used in equation (115). Since $p$ is independent of the molecular distribution of velocities (Section 8), and $P_{n}$ is obviously so by nature, the dependence of the factor $\kappa_{c} / A$ in equation (115) on this distribution is represented in equations (114) and (113) by factors involving the quantities $n, \kappa_{c}, b$, and $V_{t}$. The uncorrected values of these quantities may therefore be used in these equations. It may be noted here that the quantity, $S_{\vartheta}$ is probably very approximately, if not altogether, independent of the distribution of molecular velocities. This follows according to Section 13 from the fact that it depends mainly on the change in kinetic energy and ehange in potential energy of attraction of a gram of substance during a degree change of temperature, both changes being independent of the distribution of molecular velocities.

The value of $\kappa_{c}$ in these equations for a given substance is determined by applying equation (113) to the substance 
in the perfectly gaseous state, which corresponds to $v=\infty$, in which case the equation becomes

$$
C=n m_{a} v \kappa_{c} S_{g}=\kappa_{c} S_{g} \sqrt{\frac{R T m}{3}}, \quad . \quad .
$$

by means of equation (21), where $S_{\theta}$ is now equal to the specific heat at constant volume. The quantity $\kappa_{c}$, it should be noted, is not identical with the quantity $\kappa_{\eta}$. It is a characteristic constant depending on the molecular forces and volume of the substance.

The values of the quantities $n, V_{\imath}, b, P_{n}, p$, and $S_{o}$ are affected to a certain extent by the influence of the molecules of the substance on two molecules while interacting, or influenced by molecular interference. The quantity $\Phi_{c}$ therefore represents the portion of a similar effect on the heat conductivity of a substance which is not represented by the foregoing quantities in equations (113), (114), and (115). These equations may now be used without referring to the representative substance.

It follows similarly as in Section 34 in connection with the quantity $\Phi_{\eta}$, that the terms of the series for $\Phi_{c}$ decrease rapidly in magnitude. As a first approximation we may therefore retain only the term $\phi^{\prime}{ }_{c} / v^{2}$, or replace the series by the term $\phi_{c} / v^{x}$, where $x$ would differ little from 2 . It will readily be recognized that the quantities $\phi_{c}^{\prime}, \phi^{\prime \prime}{ }_{c}, \ldots$. , in the series for $\Phi_{c}$ are functions of $T$ and $v$, which are not very sensitive to $v$.

From equations (107) and (83) we have

$$
\frac{l_{c}}{l_{\eta}}=\frac{C}{\eta S_{g}}, \quad \cdot \quad \cdot \quad . \quad .
$$

which gives the ratio of the mean heat transfer distance associated with a molecule of a substance to the mean momentum transfer distance. This would furnish some 
information about the difference in the effect of molecular interference on the chances of a migrating molecule losing momentum or kinetic energy to a medium under the conditions of viscosity and heat conduction respectively, since these chances are respectively proportional to $1 / l_{\eta}$ and $1 / l_{c}$.

On substituting for $l_{c}$ and $l_{v}$ in the foregoing equation from equations (111) and(88), it becomes after rearranging

$$
\frac{1+\Phi_{c}}{1+\Phi_{\eta}}=\frac{\kappa_{\eta}}{\kappa_{c}} \frac{C}{\eta S_{\vartheta}}
$$

The quantities $\kappa_{\eta}$ and $\kappa_{c}$ in this equation may be determined from the viscosity and heat conduction of the substance in the gaseous state, while the quantities $C, S_{g}$, and $\eta$ (which refer to any state) may be determined directly. The equation may thus be used to compare the quantities $\Phi_{c}$ and $\Phi_{\eta}$. Since $\kappa_{c}$ and $\kappa_{\eta}$ do not depend on molecular interference according to their definitions, and $S_{g}$ also does not from its nature, while both $\Phi_{c}$ and $\Phi_{\eta}$ depend entirely on it, it follows from equation (118) that an increase of $\Phi_{c}$ relative to $\Phi_{\eta}$ is attended by an increase of $C$ relative to $\eta$. Thus this equation gives some information on the relative effects of molecular interference on the heat conductivity and viscosity of a substance.

A few applications of the foregoing investigation will now be given.

(a) Equation (116) gives the coefficient of heat conduction of a gas. Since $\kappa_{c}$ is independent of the density of the gas, it follows from the equation that this also holds for the quantity $C$. Experiment has demonstrated the truth of this result. It would of course cease to hold when the dimensions of the vessel in which conduction takes place are comparable with the length of the mean heat transfer distance, according to the conditions on which the deduction of the equation is based. 
The dynamical mechanism underlying this result may be illustrated by the following considerations. A molecule in a gas moving in the direction of the flow of heat transfers a certain amount of heat to the medium at the end of each transfer distance. If the concentration of the gas is halved the length of each transfer distance is doubled, while the heat transferred at the end of each transfer distance is also doubled since the temperature gradient remains the same. Since a change in molecular concentration of a gas does not alter the molecular velocities, the heat transferred per second by a molecule moving between two walls at different temperatures in the latter case is double that in the former. But since the number of molecules per cubic $\mathrm{cm}$. available for heat transference in the latter case is half the number available in the former case, the total heat transferred is the same in each case.

The value of $\kappa_{c}$ is found to increase with increase of temperature, as is the case with the similar quantity $\kappa_{\eta}$ connected with the viscosity of the substance. This is shown for a few gases by Table XVIII. The reason is undoubtedly the same as that holding in the case of viscosity, namely that the greater the velocity of a molecule the shorter the time it is under the influence of another molecule, and the smaller its chance of transferring heat energy, this chance being measured by $1 / l_{c}$, or $1 / \kappa_{c}$.

The values of $\kappa_{c}$ in the Table are not corrected for the distribution of molecular velocities since equation (116) was derived from equation (113) on substituting for $n$ the expression given by equation (21). If they are corrected according to Maxwell's law each value has to be multiplied by $\sqrt{\frac{3 \pi}{8}}$, or 1.085 , according to Section 11 . The same remarks apply to the values of the quantity $\kappa_{\eta}$ in the Table. 


\section{TABLE XVIII}

\begin{tabular}{|c|c|c|c|c|c|}
\hline \multicolumn{6}{|c|}{$\mathrm{C}_{2} \mathrm{H}_{4} . \quad m=44$} \\
\hline$t^{\circ} \mathrm{C}$ & $S_{0}$ cal. per gm. & $\mathrm{C}^{3} 0^{3} \mathrm{cal} . / \mathrm{cm} .{ }^{2} \mathrm{sec}$. & $\kappa_{c} 10^{10}$ & $\kappa_{\eta} 10^{10}$ & $\kappa_{c} / \kappa_{\eta}$ \\
\hline 0 & .3245 & .0395 & 2.64 & 2.08 & 1.27 \\
\hline 100 & .3403 & .0506 & 2.77 & 2.38 & 1.16 \\
\hline \multicolumn{6}{|c|}{$\mathrm{CO}_{2 .} \quad m=28$} \\
\hline 0 & .1541 & .0307 & 3.45 & 2.42 & 1.43 \\
\hline 100 & .1724 & .0506 & 4.36 & 2.92 & 1.49 \\
\hline \multicolumn{6}{|c|}{$\mathrm{H}_{2} . \quad m=2$} \\
\hline-150 & 2.41 & .1175 & 5.91 & 5.86 & 1.00 \\
\hline 0 & 2.41 & .3270 & 11.04 & 6.69 & 1.65 \\
\hline \multicolumn{6}{|c|}{ A. $\quad m=39.9$} \\
\hline 0 & .07379 & .03894 & 9.61 & 3.83 & 2.51 \\
\hline \multicolumn{6}{|c|}{ Hg. $\quad m=200.4$} \\
\hline 203 & .01476 & .01846 & 7.70 & 1.78 & 4.33 \\
\hline
\end{tabular}

On applying equations (118) and (117) to the gaseous state it follows that

$$
\frac{l_{c}}{l_{\eta}}=\frac{\kappa_{c}}{\kappa_{\eta}}=\frac{C}{\eta S_{o}}, \ldots . .
$$

since $\Phi_{\eta}=0$, and $\Phi_{c}=0$, under these conditions. The calculation of the ratio $\kappa_{c} / \kappa_{\eta}$ for different gases shows that it 
is not equal to unity, or the quantities $\kappa_{c}$ and $\kappa_{\eta}$ are not equal to each other, and that the value of the ratio depends on the nature of the gas and its temperature, as is shown by Table XVIII. We would expect that these quantities should not be equal to each other since they do not mean exactly the same thing. For $1 / l_{c}$, or $1 / \kappa_{c}$, is a measure of the chance of heat energy being transferred by a migrating molecule to its medium under the conditions of heat conduction, while $1 / l_{\eta}$, or $1 / \kappa_{\eta}$, is a measure of the chance of momentum being transferred to the medium under the conditions of viscosity. The former chance is smaller than the latter in gases, since according to the Table $\kappa_{c} / \kappa_{\eta}$, or $\frac{l_{c}}{l_{\eta}}$ is greater than unity.

It will be of interest to give a more definite and direct physical significance of $\kappa_{c}$, and compare it with that obtained for $\kappa_{\eta}$ in Sub-section (a) of Section 34. The quantity $C$ represents the amount of energy which flows per second across each square $\mathrm{cm}$. of a plane in a substance situated at right angles to a unit temperature gradient. Since $n$ molecules cross the plane per square $\mathrm{cm}$. per second in each direction, $C / n$ represents the energy conveyed by a molecule across the plane on crossing it and recrossing it (sometime) in the opposite direction. According to equations (105) and (111) this energy is equal to $\kappa_{c} S_{m} v$ in the case of a gas, where the value of $S_{m}$ is independent of the pressure of the gas. Thus $\kappa_{c}$ represents the energy conveyed by a molecule per unit of its specific heat at unit volume of a gram molecule of the gas. The quantity $\kappa_{\eta}$, we have seen, represents the momentum conveyed by a molecule across a plane at right angles to unit velocity gradient per unit mass of the molecule at unit volume of a gram molecule of the gas.

It can be shown similarly as in Section 34 that a molecule takes a time proportional to $v / V$ in crossing and recrossing a plane. The amount of energy conveyed by a 
molecule per second across a plane under the foregoing conditions is therefore proportional to $\kappa_{c} S_{m} V$, and thus independent of the volume of the gas.

(b) Equation (117) may immediately be applied to liquids to obtain the values of the ratio $l_{c} / l_{\eta}$. Table XIX

TABLE XIX

\begin{tabular}{|c|c|c|c|c|c|}
\hline Substance. & $t^{\circ} \mathrm{C}$ & $S_{\sigma}$ cal. per gm. & $\eta$ & $C$ cal./cm.$^{2} \mathbf{s e c}$ & $l_{c} / l_{\eta}$ \\
\hline E. bromide. & 15 & .2135 & .004212 & $.0{ }_{3} 247$ & .275 \\
\hline E. iodide... & 15 & .1641 & .006231 & $.0_{3} 222$ & .217 \\
\hline Chloroform. . & 15 & .237 & .006019 & $.0_{3} 288$ & .202 \\
\hline M. alcohol. ... & 15 & .5868 & .006429 & .03495 & .131 \\
\hline C. tetrachloride & 0 & .2010 & .01351 & $.0_{3} 2664$ & .0981 \\
\hline C. disulphide . . & 15 & .242 & .003905 & $.0_{3} 343$ & .363 \\
\hline Benzene....... & 10 & .4066 & .007631 & $.0_{3} 333$ & .107 \\
\hline
\end{tabular}

contains the values for a number of substances. It will be seen that they depend on the nature of the substances, and that they are smaller than unity. We have just seen that when the substances are in the gaseous state this ratio is greater than unity. This difference is due to the effect of the attraction of the molecules of a substance on a migrating molecule increasing with the density of the substance. It indicates that an increase of molecular interference increases to a greater extent the chance of a molecule transferring heat energy than of transferring momentum under the conditions of heat conduction and viscosity respectively, since these chances are measured by $1 / l_{c}$ and $1 / l_{\eta}$.

According to the foregoing result, and the result that $\kappa_{c} / \kappa_{\eta}$ is greater than unity, it follows from equation (118) and (117) that $\frac{1+\Phi_{c}}{1+\Phi_{\eta}}$ is less than unity, and that therefore $\Phi_{\eta}>\Phi_{c}$, or the interference function of viscosity is greater than the interference function of heat conduction. Since each of these quantities is zero for matter in the gaseous. 
state, an increase in the molecular interference as brought about by an increase of the density of the matter already in a dense state would therefore increase $\Phi_{\eta}$ to a greater extent than $\Phi_{c}$. Therefore according to equation (118) and the considerations following it the value of $\eta$ is increased to a greater extent by the increase of molecular interference brought about by an increase or density of the matter than the value of $C$.

(c) Equation (115) may be used to obtain the value of $\Phi_{c}$ for a liquid, and hence the corresponding value of $\phi_{c}^{\prime}$ may be deduced. The value of $P_{n}+p$ required for the calculations may be obtained from the coefficients of expansion and compression of the liquid according to Section 21 . It may also be obtained by the method of Section 46 . The value of $\kappa_{c}$ may be determined by means of equation (116), which applies to the substance in the gaseous state. At present the data are not sufficient to permit such a calculation being carried out for a substance.

(d) If the values of $C$ and $S_{g}$ be known for three different densities of a substance not differing much from each other, and the value of $\kappa_{c}$ be calculated by means of equation (116), the values of $b$ and $\phi^{\prime}{ }_{c}$ may be determined by means of a modified form of equation (113) obtained on substituting for $\Phi_{c}$ the approximate expression $\phi_{c}^{\prime} / v^{2}$, and for $n$ the expression given by equation (66) retaining only the first two terms. The equation will contain the three constants, $b, C_{2}$, and $\phi^{\prime} c$, which may be determined by applying the equation to the substance at three different densities. This operation also determines incidentally the quantities $n$ and $V_{t}$ similarly as in Section 29 .

39. Formula for the Coefficient of Conduction of Heat of a Mixture of Substances.

It will be evident from a consideration of the investigation in the previous Section that in the case of a mixture 
the heat conductivity is equal to the sum of the effective conductivities of the constituents. Hence in the case of a mixture of molecules $r$ and $e$ we may according to equation (105), write for the coefficient of conductivity

$$
C=n_{r} S_{m r} l_{c r}+n_{e} S_{m e} l_{c e}, \quad . \quad . \quad .
$$

where $S_{m r}$ and $S_{m e}$ denote the partial molecular specific heats of a molecule $r$ and $e$ respectively (Section 13), $l_{c r}$ denotes the mean heat transfer distance of a molecule $r$, $l_{c e}$ that of a molecule $e$, and $n_{r}$ and $n_{e}$ have the usual meanings. Similarly as in Section 35 we may write

$$
l_{c r}=\frac{v_{r}}{v_{r}-b_{r}^{\prime}} \frac{\kappa^{\prime}{ }_{r \tau} N}{N_{e r}}\left(1+\Phi^{\prime}{ }_{c r}\right), \ldots . .
$$

and

$$
l_{c e}=\frac{v_{e}}{v_{e}-b_{e}^{\prime}} \frac{\kappa_{c e}^{\prime} N}{N_{e r}}\left(1+\Phi_{c e}^{\prime}\right), . . . .
$$

where the symbols have similar meanings, that is, $v_{r}$ denotes the volume of the mixture containing a gram molecule of molecules $r, N_{e r}$ the total concentration of the molecules $e$ and $r_{r}, b^{\prime}{ }_{r}$ the apparent molecular volume of the mixture which appears to obstruct the motion of the molecules $r$, $\kappa^{\prime}{ }_{c r}$ the characteristic factor of the transfer path of a molecule $r$ when the mixture is in the gaseous state, and $\Phi_{c r}^{\prime}$ the heat conductivity interference function with respect to a migrating molecule $r$, while the other symbols have similar meanings. As a first approximation we may write as before

and

$$
\Phi^{\prime}{ }_{c r}=\phi_{c r}^{\prime} \frac{N^{2}{ }_{e r}}{N^{2}}
$$

$$
\Phi_{c e}^{\prime}=\phi_{c e}^{\prime} \frac{N^{2}{ }_{e r}}{N^{2}},
$$


where $N$ denotes the number of molecules in a gram molecule of a pure substance, and $\phi_{c r}^{\prime}$ and $\phi_{c e}^{\prime}$ are functions of $T$ and $N_{e r}$ but which are insensitive to variations in $N_{e r}$. On substituting from equations (122) and (121) for $l_{c r}$ and $l_{c e}$ in equation (120) and applying it to the gaseous state, it becomes

$$
C=\left\{\frac{\xi}{\xi+1} \kappa_{c r}^{\prime} S_{g r} \sqrt{m_{r}}+\frac{1}{1+\xi} \kappa^{\prime}{ }_{c e} S_{g e} \sqrt{m_{e}}\right\} \frac{1}{N} \sqrt{\frac{R T}{3}},
$$

by the help of equation (21), and since

$$
\xi=\frac{v_{e}}{v_{r}}, \frac{N}{v_{e}}+\frac{N}{v_{r}}=N_{e r}, S_{9 r}=\frac{S_{m r}}{m_{a r}}, \text { and } S_{g e}=\frac{S_{m e}}{m_{a e}},
$$

where $S_{g r}$ and $S_{g e}$ denote the partial specific heats per gram of the molecules $r$ and $e$ respectively, $\xi$ the ratio of the number of molecules $r$ to the number of molecules $e$ whose relative molecular weights referred to the hydrogen atom are $m_{r}$ and $m_{e}$, and $N$ denotes the number of molecules in a gram molecule of a pure substance. When the mixture is in the perfectly gaseous state the values of $S_{g r}$ and $S_{g e}$ are the same as for the pure substances in the gaseous state at constant volume. Equation (123) gives a relation between the quantities $\kappa_{c r}^{\prime}$ and $\kappa_{c e}^{\prime}$ which is of use in their determination.

The various quantities contained in the foregoing equations are evidently functions of $\xi$, the ratio of the constituents. With the exception of the quantities $v_{r}, v_{e}, b_{e}^{\prime}$, and $b^{\prime}{ }_{r}$, they are not identical with the quantities contained in the equations of Section 35.

The values of $\kappa^{\prime}{ }_{c r}$ and $\kappa_{c e}^{\prime}$ may be approximately calculated from the values of $\kappa_{c r}$ and $\kappa_{c e}$ referring to the substances isolated and in the perfectly gaseous state, by means of a formula similar to equation (102) in Section 35. The values thus obtained may be checked by means of equa- 
tion (123). Similarly the values of $\phi_{c r}^{\prime}$ and $\phi^{\prime}{ }_{c e}$ may approximately be ealculated by a formula similar to equation (103). The foregoing quantities may also be determined directly by the method given in the Section mentioned.

(a) It is instructive to apply equation (120) to the conduction of heat in metals. Since the molecules of a metal are more or less in relatively rigid positions the heat energy must in the main be transferred by carriers which are able to move about more freely than the molecules. It has been supposed that these carriers consist of electrons in the free state which arise througr a spontaneous dissociation of the molecules into electrons and positively charged molecules. Equilibrium exists when the number of electrons produced in this way per second is equal to the number neutralized through combination with oppositely charged parent molecules. The state of equilibrium may therefore be expressed by the equation of mass-action.

$$
K N_{n}=N_{e}^{2},
$$

where $N_{n}$ denotes the number of neutral molecules per cubic cm., $N_{e}$ the number of electrons or positively charged molecules per cubic cm., and $K$ denotes the constant of massaction. This constant is a function of the temperature, density, and nature, of the metal.

If the molecules of the metal are designated by $r$ and the electrons by $e$ in equation (120), we have $n_{r}=0$, since the molecules are more or less in relatively fixed positions, and the equation becomes

$$
C=n_{e} S_{m e} l_{c e}
$$

The value of $l_{c e}$ is probably governed by the spacing of the molecules of the metal, since the size of an electron is very small in comparison with that of an atom. As a first approximation we may therefore take $l_{c e}$ equal to the distance of 
separation of the atoms in a metal, which is usually of the order $10^{-8} \mathrm{~cm}$. The partial specific heat $S_{m e}$ of an electron probably differs little from $\frac{1}{2} m_{a} V^{2}=2.012 \times 10^{-16} T \mathrm{ergs}$, its specific heat at constant volume in the gaseous state. Whatever its value, it cannot be greater than the specific heat per atom of the metal, which is equal to twice the specific heat it would have in the gaseous state. We may therefore with a fair degree of certainty calculate by means of equation (124) the order of magnitude of $n_{e}$, the number of electrons crossing a square $\mathrm{cm}$. per second from one side to the other in a metal. Thus for example in the case of copper we have

$$
C=.7198 \text { cal. } S_{m e}=4.808 \times 10^{-24} \text { cal., }
$$

and

which gives

$$
l_{c e}=\left(\frac{m_{a r}}{\rho}\right)^{1 / 3}=2.25 \times 10^{-8} \mathrm{~cm} .
$$

$$
n_{e}=9.15 \times 10^{30} .
$$

It is of interest to compare this number with the number of molecules crossing a square $\mathrm{cm}$. from one side to the other per second in a liquid or dense gas. Thus in the case of $\mathrm{CO}_{2}$ at $40^{\circ} \mathrm{C}$. under a pressure of 200 atmos. this number is equal to $7.8 \times 10^{26}$ according to Table VIII. The density of the $\mathrm{CO}_{2}$ under these conditions is about equal to unity.

The value of $l_{c e}$ according to the foregoing considerations would increase with increase of temperature in the case of the pure metals, since they expand with increase of temperature, and the increase may probably be taken approximately equal to the increase in the distance of separation of the atoms. $S_{m e}$ is very likely practically independent of the temperature. Therefore for the cases that $C$ decreases with increase of temperature this very likely also holds for $n_{e}$ according to equation (124). Some of the metals falling 
into this category are: lead, cadmium, iron, silver, bismuth, zinc, and tin.

Other investigations having a bearing on the electrons in a metal will be found in Section 42 .

\section{The Coefficient of Diffusion of a Substance.}

If the constituents of a mixture of substances are not evenly distributed, diffusion from one part to another will take place till equilibrium is obtained. The primary

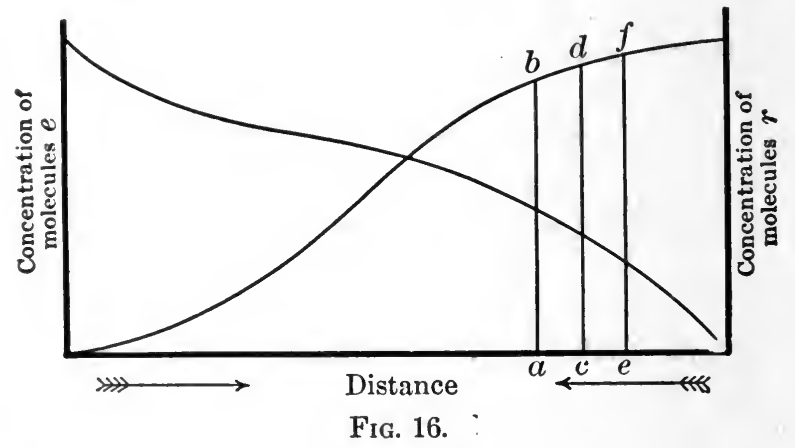

cause of diffusion is the motion of translation of the molecules. This will at once appear from the consideration of a mixture whose constituents $r$ and $e$ are distributed according to the curves shown in Fig. 16, the concentration being supposed uniform in planes parallel to the concentration axes. Molecules are projected from each element of volume of the mixture. The portion of the mixture between the planes $a b$ and $c d$ will therefore receive more molecules $r$ from the portion of the mixture between the planes $c d$ and ef, on account of the concentration gradient of the molecules $r$, than vice versa. Thus on the whole a migration of molecules $r$ in the direction of decrease of concentration would take place. Similarly it follows that the molecules 
$e$ on the whole migrate in the direction of decrease of concentration of the molecules $e$, or in the opposite direction to the molecules $r$. If $\delta_{r}$ denote the number of molecules $r$ which on the whole are transported across a square $\mathrm{cm}$., and $\frac{\delta N_{c}}{\delta x}$ denotes the concentration gradient, we have

$$
\delta_{r}=D_{r} \cdot \frac{\delta N_{c}}{\delta x}, \ldots . \quad \text {. . . . }
$$

where $D_{r}$ denotes the coefficient of diffusion of the molecules $r$. When the concentration gradient is unity $D_{r}=\delta_{r}$. It is evident that the coefficient of diffusion should depend on the density of the mixture, the ratio of the masses of the constituents, and the concentration gradient, at the point the coefficient is measured. It is found, however, to be approximately independent of the concentration gradient over a considerable range of gradients.

\section{The Mean Diffusion Path of a Molecule in} a Mixture.

The path of a molecule in a mixture in migrating from one place to another is undulatory in character on account of the interaction between the molecules due to the existence of molecular forces and molecular volume. It differs the more from a number of straight lines of various lengths joined consecutively together at various angles, the greater the density of the substance. The effect of this path on the rapidity of the diffusion of a molecule from one place to another may be represented by another path along which the molecule is supposed to move, consisting of straight lines joined together and lying near the path, and incidentally intersecting it, as shown by Fig. 14. In supposing that a molecule passes along its representative path it is obvious of course that in general the molecule at any instant does not occupy its actual position. But this does 
not matter when we are dealing with the behavior of a large number of molecules as a whole. The straight lines constituting the representative path will be called the diffusion free paths of the molecule. The average length of these paths is left arbitrary to a certain extent by their definition, and we will therefore impose the conditions that the sum of the representative free paths between any two points a considerable distance apart is equal in length to the corresponding actual path, and that each direction of a free path of given length is equally probable. These conditions completely determine the magnitude of the mean free path, as will appear from the next Section. These paths are not equal in magnitude but are grouped about the mean free path $l_{\delta}$ according to the law given in Section 31 .

\section{Formula Expressing the Coefficient of Dif- fusion in Terms of Other Quantities.}

Let us consider a heterogeneous mixture of molecules $r$ and $e$ in which the molecules are uniformly distributed in planes parallel to the plane $A B$ in Fig. 15, but non-uniformly distributed at right angles to the plane so that a diffusion of molecules $r$ towards the parallel plane $C D$ takes place, while a diffusion of molecules $e$ takes place in the opposite direction. We may suppose, to simplify the reasoning, that the molecular paths which cut the plane $E F$ in migrating towards the plane $C D$, begin their journey in the former plane at the same point. If $n_{r}$ denote the total number of molecules $r$ crossing the plane $E F$ per square $\mathrm{cm}$. from one side to the other, which is equal to the number of times the plane is cut by the corresponding free paths, the number whose paths make an angle $\theta$ with a perpendicular to the plane is similarly, as in Section 34, given by

$$
n_{r} \sin \theta \cdot d \theta \text {. }
$$


- The number of the foregoing molecules whose paths lie between $z$ and $z+d z$ is equal to

$$
n_{r} \sin \theta \cdot d \theta \cdot \frac{z}{l_{\delta r}^{2}} e-\overline{l_{\delta r}} \cdot d z,
$$

according to Section 31, where $l_{\delta r}$ denotes the mean diffusion path of the molecules $r$. This number of molecules, and the corresponding number of molecules moving in the opposite direction, which we may suppose begin their journey in the plane $G H$ in Fig. 15, are respectively proportional to the molecular concentrations of the molecules $r$ in the planes $E F$ and $G H$. If $N_{r}$ denotes the concentration in the plane $E F$, the concentration in the plane $G H$ is evidently $N_{r}-z \cos \theta \cdot \frac{d N_{r}}{d x}$, where $\frac{d N_{r}}{d x}$ is the concentration gradient measured in the direction of increase of concentration. Therefore the loss in molecules $r$ in the plane $E F$ is equal to the difference in the foregoing concentrations divided by $N_{r}$, and multiplied by the preceding number of molecules, which gives for the loss

$$
\frac{1}{N_{r}} \frac{d N_{r}}{d x} n_{r} \sin \theta \cos \theta \frac{z^{2}}{l^{2}{ }_{\delta r}} e^{-\frac{z}{l \delta r}} \cdot d z \cdot d \theta .
$$

The total loss of molecules $r$ in the plane is obtained by integrating the foregoing expression from 0 to $\infty$ with respect to the free path $z$, and from 0 to $\pi / 2$ with respect to the angle $\theta$. On referring to similar integrals in Sections 34 and 38 it will be evident that the integral in question is equal to

$$
\frac{n_{r} l_{\delta r}}{N_{r}} \frac{d N_{r}}{d x}
$$

Similarly it can be shown that the gain of molecules $e$ in the plane $E F$ is equal to

$$
\frac{n_{e} l_{\delta e}}{N_{e}} \frac{d N_{e}}{d x}
$$


where the concentration gradient of the molecules $e$ is measured in the direction of their increase of concentration, and thus in the opposite direction of the gradient of the molecules $r$. The total loss of molecules $r$ and $e$ in the plane $E F$ is therefore equal to

$$
\frac{n_{r} l_{\delta r}}{N_{r}} \frac{d N_{r}}{d x}-\frac{n_{e} l_{\delta e}}{N_{e}} \frac{d N_{e}}{d x}
$$

which may be written $M_{r}-M_{e}$. When the molecular paths are in their original positions the foregoing number represents, on the whole, the gain in molecules on the lower side of the plane $E F$. But the space occupied by these molecules is not zero. A portion of the mixture must therefore be transported bodily across the plane in the opposite direction to make room for the foregoing molecules. Let $P_{v}$ denote the volume of this portion of the mixture. The total number of molecules $r$ transported on the whole across the plane per square $\mathrm{cm}$. in the direction of decrease of concentrtaion gradient, which is equal to the diffusion $\delta_{r}$, is accordingly given by

$$
\delta_{r}=\frac{n_{r} l_{\delta r}}{N_{r}} \frac{d N_{r}}{d x}-\frac{P_{v} N_{r}}{N_{e}+N_{r}}
$$

Let $\vartheta_{r}$ and $\vartheta_{e}$ denote the external molecular volumes of a molecule $r$ and of a molecule $e$ respectively in the mixture, i.e., the decrease in the volume of the mixture when a molecule $r$ or $e$ at constant pressure is removed. These quantities are evidently connected by the equation

$$
N_{r} \vartheta_{r}+N_{e} \vartheta_{e}=1 \text {. }
$$

The quantity $P_{v}$ is therefore given by the equation

$$
\frac{P_{0} N_{r} \vartheta_{r}}{N_{r}+N_{e}}+\frac{P_{0} N_{e} \vartheta_{e}}{N_{r}+N_{e}}=M_{r} \vartheta_{r}-M_{e} \vartheta_{e},
$$


or

$$
P_{v}=\left\{\frac{M_{r} \vartheta_{r}-M_{e} \vartheta_{e}}{N_{r} \vartheta_{r}+N_{e} \vartheta_{e}}\right\}\left(N_{r}+N_{e}\right) .
$$

On substituting this expression for $P_{0}$ in the preceding diffusion equation, and then substituting the expressions for $M_{e}$ and $M_{r}$, the equation becomes

$$
\delta_{r}=\frac{\vartheta_{e}}{N_{r} \vartheta_{r}+N_{e} \vartheta_{\epsilon}}\left\{\frac{n_{r} l_{\delta r} N_{e}}{N_{r}} \frac{d N_{r}}{d x}+\frac{n_{e} l_{\delta e} N_{r}}{N_{e}} \frac{d N_{e}}{d x}\right\}, .
$$

which is a fundamental form of the diffusion equation. The rate of diffusion of the molecules $e$, which takes place in the opposite direction to the molecules $r$, is obtained on interchanging the suffixes $r$ and $e$ in the foregoing equation.

It will readily be seen that the rates of diffusion for the two different kinds of molecules may be written $\delta_{r}=K \vartheta_{e}$, and $\delta_{e}=K \vartheta_{r}$, and hence the ratio of the rates of diffusion is given by

$$
\frac{\delta_{r}}{\delta_{e}}=\frac{\vartheta_{e}}{\vartheta_{r}} . . . . . . .
$$

In the case of a liquid mixture the quantities $\vartheta_{r}$ and $\vartheta_{c}$ are not equal to each other. They may easily be measured in practice since

$$
\vartheta_{r}=\frac{\delta v}{\delta N_{r}}, \text { and } \vartheta_{e}=\frac{\delta v}{\delta N_{e}}
$$

where $v$ denotes the volume of the mixture.

In the case of a gaseous mixture $\vartheta_{e}=\vartheta_{r}$, and $N_{e}+N_{r}=$ $C_{e r}$ a constant since the pressure is everywhere the same. Equation (126) in that case becomes

$$
\delta_{r}=\frac{1}{N_{r}+N_{e}}\left\{\frac{n_{r} l_{\delta r} N_{e}}{N_{r}}+\frac{n_{e} l_{\delta e} N_{r}}{N_{e}}\right\} \frac{d N_{r}}{d x} . .
$$

If the concentration of one of the constituents of the mixture is small in comparison with that of the other, say 
of the molecules $r$ in comparison with the molecules $\boldsymbol{e}$, equation (126) becomes

$$
\delta_{r}=\frac{n_{r} l_{\delta r}}{N_{r}} \frac{d N_{r}}{d x}
$$

The rate of diffusion is then independent of $\vartheta_{r}$ and $\vartheta_{e}$.

The forms of the foregoing equations may be modified by considering similarly as in the four previous Sections a representative mixture which has the same rate of diffusion, expansion pressure, and molecular volumes $\vartheta_{r}$ and $\vartheta_{e}$, but whose molecules do not possess an apparent molecular volume $b$ whose defining property according to Section 19 is that a change in its value produces a change in the external pressure without changing the total average velocity. It should be carefully noted that the quantities $b$ and $\vartheta$ do not mean the same thing, one may be zero when the other is not. The former quantity represents the obstruction the molecules present to each other's motion, while the latter quantity represents the change in the external volume of a mixture on adding a molecule. It will be convenient now to replace the volume $v$ by the concentration $N_{e r}$ similarly as in Sections 35 and 39 . The molecular free paths of the molecules $r$ and $e$ may then be expressed similarly as before by the equations

and

$$
\left.\begin{array}{l}
l_{\delta r}=\frac{v_{r}}{v_{r}-b^{\prime}{ }_{r}} \frac{N \kappa_{\delta r}^{\prime}}{N_{e r}}\left(1+\Phi_{\delta r}^{\prime}\right) \\
l_{\delta e}=\frac{v_{e}}{v_{e}-b_{e}^{\prime}{ }_{e}} \frac{N \kappa_{\delta e}^{\prime}}{N_{e r}}\left(1+{\left.\Phi^{\prime}{ }_{\delta e}\right)}^{\prime}\right.
\end{array}\right\} \cdot \cdot \cdot \cdot
$$

where $v_{r}$ denotes the volume of the mixture containing a gram molecule of molecules $r, N_{e r}$ the total concentration of the molecules $r$ and $e, b^{\prime}{ }_{r}$ the apparent molecular volume of the mixture which appears to obstruct the motion of the molecules $r, \kappa^{\prime}{ }_{\delta r}$ the characteristic factor of the diffusion 
free path of a molecule $r$ when the mixture is in the gaseous state, and $\Phi^{\prime}{ }_{\delta r}$ the diffusion interference function with respect to a migrating molecule $r$, while the other symbols have similar meanings. As a first approximation we may write similarly as before

$$
\begin{aligned}
& \Phi_{\delta r}^{\prime}=\phi_{\delta r}^{\prime} \frac{N_{e r}^{2}}{N^{2}}, \\
& \Phi_{\delta e}^{\prime}=\phi_{\delta e}^{\prime} \frac{N_{e r}^{2}}{N^{2}},
\end{aligned}
$$

where $N$ denotes the number of molecules in a gram molecule of a pure substance, and $\phi^{\prime}{ }_{\delta r}$ and $\phi^{\prime}{ }_{\delta e}$ are functions of $T$ and $N_{e r}$, but which are insensitive to variations in $N_{e r}$.

.On applying equations (130) to the gaseous state they become

$$
l_{\delta r}=\kappa^{\prime}{ }_{\delta r} \frac{N}{N_{e r}},
$$

and

$$
l_{\delta e}=\kappa^{\prime}{ }_{\delta e} \frac{N}{N_{e r}} .
$$

Substituting for $l_{\delta r}$ and $l_{\delta e}$ from these equations in equation (128) and obtaining expressions for $n_{r}$ and $n_{e}$ by means of equation (21) the equation becomes

$$
\delta_{r}=\frac{N}{N_{e r}^{2}} \sqrt{\frac{R T}{3}}\left\{\frac{N_{e \kappa^{\prime}}{ }^{\prime}}{\sqrt{m_{r}}}+\frac{N_{r \kappa^{\prime}}{ }_{\delta e}}{\sqrt{m_{e}}}\right\} \frac{d N_{r}}{d x}, \cdots
$$

since $N_{e}+N_{r}=N_{e r}, v_{r} m_{a r} N_{r}=m_{r}$, and $v_{e} m_{a e} N_{e}=m_{e}$, where $m_{a r}$ and $m_{a e}$ denote the absolute and $m_{r}$ and $m_{e}$ the relative molecular weights of the molecules $r$ and $e$ respectively. This equation applies to the gaseous state and gives the relation between the characteristic quantities $\kappa_{\delta r}^{\prime}$ and $\kappa^{\prime}$ se which are functions of the temperature and the nature of the molecules. 
Since $l_{\delta r}=\kappa^{\prime}{ }_{\delta r} N / N_{c r}$ when the mixture is in the gaseous state, it will be evident from an inspection of Fig. 14, which shows the relation between the free paths of a molecule and its actual path, that $1 / l_{\delta r}$, or $1 / \kappa^{\prime}{ }_{\delta r}$ is a measure of the chance of the resultant force on a migrating molecule passing through a maximum or minimum and changing its direction.

When the mixture is in the gaseous state and the concentration of one set of molecules is relatively small, another interesting significance may be attached to the quantity $\kappa_{\delta r}^{\prime}$. Equation (129) then applies, and it indicates that for the same concentration gradient per molecule, or $\frac{1 d N_{r}}{N_{r} d x}=$ constant, the foregoing quantity is proportional to $\delta_{r} / n_{r}$. Therefore, since $n_{r}$ denotes the number of molecules crossing per second per square $\mathrm{cm}$. a plane situated at right angles to the direction of diffusion, and $\delta_{r}$ denotes the rate the molecules diffuse across the plane, the quantity $\kappa_{\delta r}^{\prime}$ is a measure of the chance of a molecule which has crossed the plane from one side to the other, to remain on the latter side.

The quantities $\Phi^{\prime}{ }_{\delta r}$ and $\Phi^{\prime}{ }_{\delta e}$ in the foregoing equations express the effect of the molecular interference of the mixture on two interacting molecules in changing the value of $\delta_{r}$, which is not expressed by the other quantities which are similarly affected.

The values of $n_{r}, n_{e}, b_{r}^{\prime}$, and $b^{\prime}{ }_{e}$ in the foregoing equations may be found by the methods of Section 29, or by those given in Section 46. The values thus obtained may be corrected according to Maxwell's distribution of molecular velocities in the way described. The values of the other quantities which cannot be measured directly may be determined by methods which will now be described.

In the case of a dilute solution of molecules $r$ in $e$ the value of $l_{\delta r}$ is immediately given by equation (129) since $\delta_{r}$ may be measured directly. If the coefficient of diffusion 
for the same kind of mixture in the gaseous state were measured, or otherwise were known, the value of $\kappa^{\prime}{ }_{\delta r}$ would be given by equation (133) to which equation (129) can be reduced. The value of $\Phi^{\prime}{ }_{\delta r}$ could then be obtained from equations (130).

In the case of a mixture in which one of the constituents is not small in comparison with the other the quantities $\kappa^{\prime}{ }_{\delta r}$ and $\kappa^{\prime}{ }_{\delta e}$ may be determined by means of equation (131). Thus we may write

$$
\frac{1}{\kappa_{\delta r}^{\prime}}=\frac{1}{N_{e r}}\left\{N_{r} a_{r}+N_{e} a_{r e}\right\}
$$

and

$$
\frac{1}{\kappa^{\prime} \delta e}=\frac{1}{N_{\iota r}}\left\{N_{e} a_{e}+N_{r} a_{r e}\right\}
$$

similarly as in Section 35, and substitute from these equations for the foregoing quantities in equation (131). The three constants $a_{r}, a_{e}$, and $a_{r e}$, at constant temperature may be determined by applying the resultant equation to three diffusing gaseous mixtures of different relative concentrations, which furnishes three simultaneous equations. The values of $\kappa^{\prime}{ }_{\delta r}$ and $\kappa^{\prime}{ }_{\delta e}$ may then be calculated for any relative concentration of the molecules by means of the foregoing two equations.

An interesting special case of such calculations corresponds to $N_{e}=0$. The resultant value of $\kappa_{\delta r}^{\prime}$ may be used to calculate the coefficient of diffusion of a molecule $r$ in a gas of the same kind (a quantity which cannot be measured directly) by means of equation (131) putting $N_{e}=0$.

The values of $\kappa^{\prime}{ }_{\delta r}$ and $\kappa^{\prime}{ }_{\delta e}$ obtained by the foregoing method would not be corrected for the distribution of molecular velocities. If this correction is carried out according to Maxwell's law each value has to be multiplied by 1.085 . 
The quantities $\phi^{\prime}{ }_{\delta r}$ and $\phi^{\prime}{ }_{\delta c}$ may now be determined by means of equation (126) on substituting for $\Phi_{\delta r}^{\prime}, \Phi_{\delta r}^{\prime}$, $l_{\delta r}$, and $l_{\delta \varepsilon}$ in the equation from the equations defining these quantities, and for $\phi_{\delta r}^{\prime}$ and $\phi_{\delta e}^{\prime}$ from the equations

$$
\phi_{\delta r}^{\prime}=\frac{1}{N_{e r}^{2}}\left\{b_{r} N_{r}^{2}+b_{r r e} N_{e} N_{r}+b_{r e e} N_{e}^{2}\right\},
$$

and

$$
\phi^{\prime}{ }_{\delta e}=\frac{1}{N_{e r}^{2}}\left\{b_{e} N_{e}^{2}+b_{r e t} N_{e} N_{r}+b_{r r e} N_{r}^{2}\right\},
$$

deduced similarly as similar equations in Section 35 . An equation is obtained containing the four constants $b_{e}, b_{r}$, $b_{r e e}$, and $b_{r r e}$ at constant temperature. Therefore on applying the equation to four dense diffusing mixtures of different relative concentrations of the molecules $r$ and $e$ four simultaneous equations will be obtained from which these constants can be determined. The values of $\phi_{\delta r}^{\prime}$ and $\phi_{\delta e}^{\prime}$ may then be calculated for any relative concentration of the molecules $r$ and $e$ in the mixture by means of the foregoing equations. An interesting special case of such a calculation is that corresponding to $N_{e}=0$.

The number of times $N_{\delta}$ a molecule passes over its mean free diffusion path $l_{\delta}$, which is inversely proportional to the number of times the force on a molecule passes through a maximum and changes its direction of motion, is given by

$$
N_{\delta}=\frac{V_{t}}{l_{\delta}}, \quad \text {. . . . . . }
$$

where $V_{t}$ denotes the total average velocity of a molecule. This equation follows from the fact that the representative path of a molecule is equal in length to the actual path.

(a) As an application of the foregoing investigation let us consider the diffusion of molecules $r$ and $e$ in the gaseous state into each other. If the concentration of the mole- 
cules $r$ is small in comparison with that of the molecules $e$ the coefficient of diffusion $D_{r}$ of the molecules $r$ is according to equations (125), (129), and (130), given by

$$
D_{r}=\frac{N n \kappa_{\delta r}^{\prime}}{N_{r} N_{e r}} \text {. . . . . . . }
$$

Now according to equations (35) and (8)

$$
n_{r}=\frac{N_{r} V_{t r}}{3}=\frac{N_{r}}{3} \sqrt{\frac{3 R T}{m_{r}}},
$$

and the foregoing equation may therefore be written

$$
D_{r}=\frac{N \kappa^{\prime}{ }_{\delta r}}{N_{e r}} \sqrt{\frac{R T}{3 m_{r}}} . \quad \text {. . . . . . . }
$$

The coefficient of diffusion is therefore inversely proportional to the total molecular concentration, since $\kappa^{\prime}{ }_{\delta r}$ is independent of the volume of the mixture. This result is borne out by experiment.,

The foregoing equation may also be written

$$
D_{r}=4.377 \times 10^{77} \frac{\kappa_{\delta r}^{\prime} T}{p} \sqrt{\frac{T}{m_{r}}} . \quad .
$$

since $N_{e r}=7.46 \times 10^{15} p / T$ according to equation (15), $N=6.2 \times 10^{23}$, and $R=8.315 \times 10^{7}$, where $p$ denotes the external pressure. This equation was used to calculate the values of $\kappa_{\delta}$ given in Table XX for the inter-diffusion of a number of different gases, where for convenience and simplicity $\kappa_{\delta}$ is now written for $\kappa_{\delta r}^{\prime}$, the values of $D$ used corresponding to $p=760 \mathrm{~cm}$. of mercury. The directions of the diffusion to which the coefficients in the Table refer are indicated by arrow heads.

It will be seen on inspecting the table that $\kappa_{\delta}$ increases with increase of temperature for the same two diffusing gases. The chance of the resultant force on a migrating 
molecule per unit length of path passing through a maximum and changing in direction is thus decreased by an increase of temperature. Actually this means that some of the smaller maxima or bends of the molecular path are more or less smoothed out by an increase of temperature. This would happen because the time any pair of interacting molecules are under each other's influence is decreased by an increase of temperature, since this increases the molecular velocities, and hence the amount of deflection each molecule undergoes is decreased.

TABLE XX

\begin{tabular}{|c|c|c|c|c|}
\hline$t^{\circ} \mathrm{C}$ & $D$ & $\kappa_{\delta} 10^{10}$ & $D$ & $\kappa_{\delta} 10^{10}$ \\
\hline & \multicolumn{2}{|c|}{$\mathrm{H}_{2} \mathrm{O} \rightarrow \mathrm{CO}_{2}$} & \multicolumn{2}{|c|}{$\mathrm{H}_{2} \mathrm{O} \rightarrow \mathrm{H}_{2}$} \\
\hline \multirow{3}{*}{$\begin{array}{l}0 \\
92.4\end{array}$} & .132 & 2.84 & .687 & 14.8 \\
\hline & .2384 & 3.31 & 1.179 & 16.3 \\
\hline & \multicolumn{2}{|c|}{$\mathrm{CH}_{4} \mathrm{O} \rightarrow \mathrm{CO}_{2}$} & \multicolumn{2}{|c|}{$\mathrm{CH}_{4} \mathrm{O} \rightarrow \mathrm{H}_{2}$} \\
\hline \multirow{3}{*}{$\begin{array}{c}0 \\
49.6\end{array}$} & .0880 & 2.52 & .5001 & 14.3 \\
\hline & .1234 & 2.75 & .6738 & 15.0 \\
\hline & \multicolumn{2}{|c|}{$\mathrm{C}_{6} \mathrm{H}_{6} \rightarrow \mathrm{CO}_{2}$} & \multicolumn{2}{|c|}{$\mathrm{C}_{6} \mathrm{H}_{6} \rightarrow \mathrm{H}_{2}$} \\
\hline 0 & .0527 & 2.36 & .294 & 13.1 \\
\hline \multirow[t]{2}{*}{45} & .0715 & 2.54 & .3993 & 14.2 \\
\hline & \multicolumn{2}{|c|}{$\mathrm{C}_{9} \mathrm{H}_{18} \mathrm{O}_{2} \rightarrow \mathrm{CO}_{2}$} & \multicolumn{2}{|c|}{$\mathrm{C}_{9} \mathrm{H}_{18} \mathrm{O}_{2} \rightarrow \mathrm{H}_{2}$} \\
\hline 0 & .0305 & 1.94 & .1724 & 11.0 \\
\hline 97.8 & .0568 & 2.29 & .3177 & 12.8 \\
\hline
\end{tabular}


It appears also that for the same medium the value of $\kappa_{\delta}$ decreases with an increase of molecular weight of the diffusing molecule. This indicates that the chance of the resultant force on a migrating molecule per unit length of its path passing through a maximum and changing in direction is increased by an increase in the molecular weight of the molecule. The relative change in $\kappa_{\delta}$, it will be noticed, is considerably smaller than the relative change in the molecular weight. Thus an increase in molecular weight acts in the opposite direction to an increase in temperature on the value of $\kappa_{\eta}$, as we might expect. It is evident that an increase in molecular weight increases the time the interacting molecules are under each other's influence, since this is attended by a decrease in their velocities, and the force that they exert upon each other is also increased. The values in the Table are not corrected for Maxwell's law of distribution of molecular velocities the correction corresponding to the introduction of the factor 1.085 .

If the quantities $n_{e}$ and $n_{r}$ in equation (128), which applies to the gaseous state, are expressed in terms of the corresponding molecular velocities, the equation assumes the form usually given in treatises on the Kinetic Theory of Gases. The quantities $l_{\delta r}$ and $l_{\delta e}$ are supposed to refer, however, to the free paths of molecular collision of the molecules $r$ and $e$ respectively, and therefore have not the same fundamental meaning given to them in this book.

(b) An interesting application of equation (129) may be made in connection with the electrons in a metal. We may write $N_{e}=K_{e} n_{e}$, where $N_{e}$ denotes the number of free electrons per cubic $\mathrm{cm}$. in a metal, and $n_{e}$ the number crossing a square $\mathrm{cm}$. from one side to the other in all directions per second. A change in $N_{e}$ evidently produces a change in $n_{e}$ through the increase in concentration of the electrons and the change in their interaction upon each other. 
It will not be difficult to see that the change in $n_{e}$ from the former cause is large in comparison with that due to the latter when the change in concentration is small. We may therefore consider $K_{e}$ a constant for small changes of $N_{e}$. Equation (129) applied to the diffusion of electrons in a metal may therefore be written

$$
\delta_{e}=l_{\delta e} \frac{d n_{e}}{d x} .
$$

Since $\frac{A}{2} n_{e}$ is equal to the partial expansion pressure $P_{e}$ of the electrons according to Section 20 the foregoing equation may be written

$$
\delta_{e}=\frac{2 l_{\delta e}}{A} \frac{d P_{e}}{d x},
$$

where $\frac{d P_{e}}{d x}$ is evidently the force acting on a cubic $\mathrm{cm}$. of diffusing electrons. We may therefore suppose that the electrons are uniformly distributed and this force produced by an electric field $X$, so that

$$
X e N_{e}=\frac{d P_{e}}{d x},
$$

where $e$ denotes the electric charge on an electron, and the equation therefore given the form

$$
\delta_{e}=\frac{2 l_{\delta e} X e N_{e}}{A} .
$$

The current $I$ produced by the electric field is given by

$$
I=e \delta_{e}=k X,
$$

where $k$ denotes the electric conductivity of the material; and the foregoing two equations therefore give

$$
N_{e}=\frac{2.543 \times 10^{-20} \mathrm{k} \sqrt{T m_{e}}}{l_{\delta e} e^{2}}, \quad \cdot .
$$


where $m_{e}$ denotes the mass of an electron relative to the hydrogen atom, and $A=5.087 \times 10^{-20} \sqrt{T m_{e}}$. This equation may be used to calculate the number of electrons per cubic $\mathrm{cm}$. of a metal:

The value of the free diffusion path $l_{\delta e}$ of an electron in a metal is probably in the main governed by the presence of the atoms, and as a first approximation may therefore be taken equal to the distance of separation of the atoms. Approximate values of $N_{e}$ may thus be obtained. Thus for example in the case of copper at $0^{\circ} \mathrm{C} . k=7.7 \times 10^{-4}$ E.M.U., and since $m_{e}=.001, e=1.6 \times 10^{-20}$ E.M.U., $T=$ $273^{\circ}$, and $l_{\delta e}=2.25 \times 10^{-8}$ approximately, we obtain

$$
N_{e}=2 \times 10^{24} \text { approximately. }
$$

Equation (136) gives definite information about the variation of $N_{e}$ with the temperature. Since $k$ for the pure metals varies approximately inversely as the absolute temperature, it follows from the equation that

$$
N_{e} \propto \frac{1}{l_{\delta e} \sqrt{\bar{T}}} .
$$

Therefore, since $l_{\delta e}$ can only increase with increase of temperature, the value of $N_{e}$ decreases with increase of temperature in the case of the pure metals. This result may of course not hold in the case of alloys whose conductivity usually increases with increase of temperature.

Experiment shows that the conductivity of a metal is greatly affected by small amounts of impurities. These should not alter the value of $l_{\delta e}$ to an appreciable extent. The change in conductivity must therefore be due according to equation (136) to a change in $N_{e}$ induced by the impurities. This is probably brought about by the impurities affecting the rate of dissociation of the atoms of the metal, and therefore the corresponding constant of mass-action. 
From equations (136) and (124) we obtain

$$
\frac{C}{k}=\frac{2.543 \times 10^{-20} n_{e} l_{c e} S_{m e} \sqrt{T m_{e}}}{N_{e} l_{\delta e} e^{2}} .
$$

This equation gives the value of the ratio $\frac{n_{e} l_{c e} S_{m e}}{N_{e} l_{\delta e}}$, since the quantities $C$ and $k$ can be measured directly, and the remaining quantities are known constants. The ratio $C / k$ has approximately the same value for all pure metals at the same temperature, and is approximately proportional to the absolute temperature $T$. This is shown by Table XXI, which was taken from Richardson's Electron Theory

\section{TABLE XXI}

\begin{tabular}{|c|c|c|}
\hline Material. & $\begin{array}{l}\text { Values of } \\
C / k \text { at } 18^{\circ} \mathrm{C} .\end{array}$ & $\begin{array}{l}\text { Temp. Coef. } \\
\text { of Ratio. }\end{array}$ \\
\hline Copper (pure)............. & $6.65 \times 10^{10}$ & $3.9 \times 10^{-3}$ \\
\hline Silver (pure) ............. & $6.86 \times 10^{10}$ & $3.7 \times 10^{-3}$ \\
\hline Gold (pure) ............... & $7.27 \times 10^{10}$ & $3.6 \times 10^{-3}$ \\
\hline Nickel (pure)............. & $6.99 \times 10^{10}$ & $3.9 \times 10^{-3}$ \\
\hline Zinc (pure) $\ldots \ldots \ldots \ldots \ldots \ldots$ & $7.05 \times 10^{10}$ & $3.8 \times 10^{-3}$ \\
\hline Cadmium (pure) .......... & $7.06 \times 10^{10}$ & $3.7 \times 10^{-3}$ \\
\hline 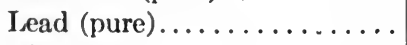 & $7.15 \times 10^{10}$ & $4.0 \times 10^{-3}$ \\
\hline Tin (pure) $\ldots \ldots \ldots \ldots \ldots$ & $7.35 \times 10^{10}$ & $3.4 \times 10^{-3}$ \\
\hline Aluminium .............. & $6.36 \times 10^{10}$ & $4.3 \times 10^{-3}$ \\
\hline Platinum (pure)............ & $7.53 \times 10^{1 n}$ & $4.6 \times 10^{-3}$ \\
\hline Palladium .............. & $7.54 \times 10^{10}$ & $4.6 \times 10^{-3}$ \\
\hline Iron $\ldots \ldots \ldots \ldots \ldots \ldots \ldots$ & $8.02 \times 10^{10}$ & $4.3 \times 10^{-3}$ \\
\hline Bismuth................ & $9.46 \times 10^{10}$ & $1.5 \times 10^{-3}$ \\
\hline
\end{tabular}

of Matter. Since $l_{c e} / l_{\delta e}$ is very probably independent of the temperature, and this is also likely to hold approximately for $S_{m e}$, the partial specific heat of the electrons, it follows that approximately

$$
\frac{n_{e}}{N_{e}} \propto T^{1 / 2} .
$$


Another expression for the electric conductivity of a metal, which is well known, may be obtained as follows: Consider a metal in which the electrons are under the action of an electric field of intensity $X$. Let $l$ denote the length of the actual path of an electron at the end of which it possesses the same amount of kinetic energy as at the beginning, being the path over which all the energy imparted to the electron by the electric field is imparted to the surrounding electrons and molecules of the metal. It will be noticed that again we define molecular path without the introduction of molecular collision, as this is more satisfactory. If $V_{t}$ as usual denotes the total average velocity (Section 17) of an electron, the time $t$ it takes to traverse the distance $l$ is given by $t=l / V_{t}$. The effect of the electric field is to give an acceleration equal to $X e / m_{a e}$ to the electron, whose absolute mass is $m_{a e}$. If we suppose that this is unimpeded along the path of the electron, it will have a component velocity equal to $\frac{X e t}{m_{a e}}$ at the end of the path. This causes a drift of the electrons which is approximately equal to $\frac{X e t^{2}}{2 m_{a e}}$ during the time $t$. The average velocity of the drift is therefore $\frac{X e t}{2 m_{a e}}$, or $\frac{X e l}{2 m_{a e} V_{i}}$. The electric current in the metal is therefore given by

$$
\frac{X e l}{2 m_{a e} V_{t}} \times N e,
$$

and accordingly the electric conductivity $k$ by

$$
k=\frac{N_{e} e^{2} l}{2 m_{a e} V_{t}}=\frac{N_{e}^{2} e^{2} l}{6 n m_{a e}}, \quad \text {. . . }
$$

by the help of equation (35). 
If the supposition is made that $l=l_{c}$, and that the electrons behave as if they were in the perfectly gaseous state, ${ }^{*}$ the ratio $C / k$ formed from equations (138) and (105) is proportional to the absolute temperature $T$, and its value agrees approximately with that found by experiment. It is hardly likely, however, that the suppositions made are true, since the theoretical ratio shows great deviations from the experimental when the metals contain small amounts of impurities, which should not affect the suppositions made. Moreover, it is obvious that in general $l$ cannot be equal to $l_{c}$. What we can say with certainty only is that the theoretical ratio of $C / k$ has a factor of $T$ which for pure metals has a value corresponding to the electrons behaving as if they were in the perfectly gaseous state, according to the results of experiment.

From equations (138) and (136) on eliminating $k$ we have

$$
V_{t}=\frac{l}{l_{\delta e}} \frac{2.545 \times 10^{-20} \sqrt{T m_{e}}}{2 m_{a e}} .
$$

This equation identically vanishes if we assume that $l=2 l_{\delta e}$, and that $V_{t}$ is given by equation (8), which corresponds to the electrons behaving as if they were in the perfectly gaseous state. Thus the latter assumption, and the assumption that $l=l_{c e}=2 l_{\delta e}$, satisfy equations (124), (136), and (138).

Accordingly the assumption that $l_{c e}=2 l_{\delta e}$, and that the electrons behave as if they were in the gaseous state, give a value for the theoretical ratio of $C / k$ expressed by equation (137) which agrees with the facts. But the assumptions are not likely to hold for the same reasons as stated previously.

* Which corresponds to $n$ being given by equation (21), $S_{m e}$ being equal to $4.808 \times 10^{-24}$ cal., and taking into account that $v N_{e} m_{a e}=m_{e}$ $=.001$. 
The expression for the ratio $C / k$ obtained from equations (124) and (138) applied to the gaseous state is the one usually given in connection with electric conductivity, but the meanings usually attached to $l$ and $l_{c e}$ are not exactly the same as those given in this book. The expression for the ratio given by equation (137) is mathematically more fundamental, as will easily be recognized.

The diffusion of gases may also be treated from another aspect, which involves the attraction of the molecules upon each other.

\section{Maxwell's Expression for the Coefficient of Diffusion of Gases.}

If a molecule may be regarded simply as a center of forces of attraction and repulsion the coefficient of diffusion of a gas into another gas is a function of the law of force between the molecules. The mathematics involved in finding the function for any given law of force is, however, of a very complicated character, and does not yield expressions of any practical use except in one case. Maxwell* has shown that if the attraction between two molecules separated by a distance $x$ may be represented by the expression $B / x^{5}$, where $B$ denotes a constant depending on the nature of the molecules, the coefficient of diffusion $D_{r}$ of a gas $r$ into a gas $e$ is given by

$$
D_{r}=\frac{p_{r}}{\rho_{\rho_{c}} A_{c}\left\{\frac{p_{e}}{m_{r} m_{e}\left(m_{r}+m_{e}\right)}\right\}^{1 / 2}} \frac{1}{p}, \ldots .
$$

where $\rho_{r}, p_{r}, \rho_{e}$, and $p_{e}$ denote the partial densities and pressures respectively of the molecules $r$ and $e$ whose molecular weights are $m_{r}$ and $m_{e}, p=p_{r}+p_{e}$, and $A_{c}$ denotes a

* "Dynamical Theory of Gases" Collected Papers, Vol. II, p. 36. 
numerical constant. The attraction between two molecules may probably over a certain region be approximately represented by a single term of the above form according to Section 26. Actually the force between two molecules according to Section 14 is expressed by a number of terms which is probably greater than three. The equation has been applied to gases by the writer * to calculate their relative coefficients of diffusion, in connection with investigations of the nature of the forces of molecular interaction. The discussion of the results is therefore reserved for another place.

In the previous Sections the diffusion, viscosity, conduction of heat, was investigated without any reference to the exact nature of the law of force of molecular interaction. The free paths introduced have not the direct physical significance associated with the paths defined by the old method of molecular collision. But we have seen that some other important physical significance can be attached to each path, and to the more important component factor $\kappa$ about which really the interest of each kind of path centers. This procedure, there can be no doubt, is more fundamental than that involving molecular collision, and moreover it is mathematically quite sound, besides being very much simpler. The constants involved in the various expressions obtained in this way are obviously functions of the law of molecular attraction and repulsion. The results obtained will be used in the next chapter to interpret various aspects of Brownian motion, and the diffusion and mobility of particles. The molecular free paths will also be given extended forms along the lines worked out in the previous Sections, by the aid of which useful and interesting information may be obtained about molecular motion under various conditions.

*Phil. Mag., May, pp. 783-809, 1910. 


\section{CHAPTER IV}

MISCELLANEOUS APPLICATIONS, CONNECTIONS, AND EXTENSIONS OF THE RESULTS OF THE PREVIOUS CHAPTERS

44. The Direct Observation of some of the Quantities depending on the Nature of the Motion of a Molecule in a Substance, and their Use.

In Section 4 we have seen that the motion of translation of a colloidal particle in a liquid may be of such a magnitude, depending on the size of the particle, that it can conveniently be observed directly by means of the ultra microscope. The motion is oscillatory in nature, and takes place in haphazard directions, giving rise to a migration of the particle which is also oscillatory and haphazard in character. If the solution is given a motion at right angles to the microscope by passing a stream of the solution through the containing vessel, the motion of each particle as it appears to the eye is the resultant of the motion of the particle and that of the liquid, and thus the particle appears to traverse an undulatory or wavy curve. This method of observation was introduced by Svedberg*, who accordingly speaks of the average amplitude $A_{1}$ and wave-length $\lambda$ of the apparent path of the particle. Fig. 17 shows as an illustration some curves that he obtained in this way. The left-hand side of the figure shows cases

*Zs. f. Electroch., 12, 1906, pp. 853-909; Zs. f. Phys. Chem., 71, 1910, p. 571. 


\section{MisCELLANEOUS APPLICATIONS, CONNECTIUNS}

of motion of particles as they would appear to the eye if the fluid were at rest, while the right-hand side shows the curves they would trace out in the plane of observation on giving a parallel motion to the fluid. If the actual motion of a particle in a liquid at rest were along a number of straight lines joined together consecutively at different angles into a continuous line, and this motion were compounded with a uniform motion in a given direction, the motion of the
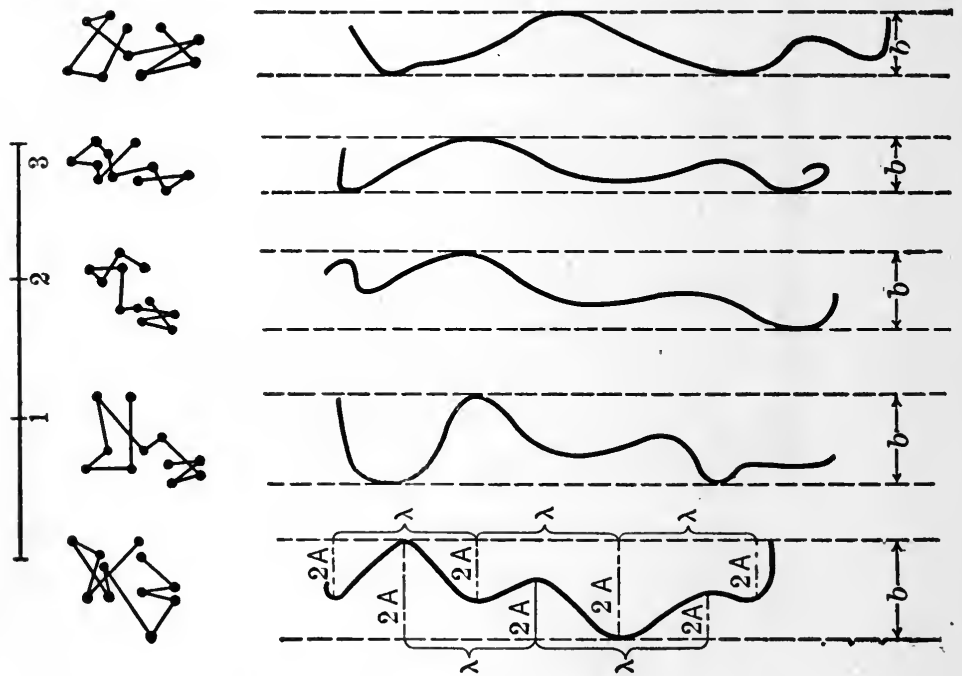

FJG. 17.

particle projected on to a plane would appear as a curve of a similar character, namely consisting of straight lines joined together at various angles. This is not observed in practice, however, and it follows therefore that the motion of a particle is not suddenly changed in direction at certain points only along its path, but continually so more or less, showing that it is continually under the influence of the surrounding molecules. 
If a point is chosen in the vicinity of each bend of the actual path of the particle, and each consecutive pair of points joined by a straight line subject to the conditions that the sum of the lengths of the lines between two points a considerable distance apart is equal in length to the corresponding path, and each direction of a line in space is equally probable, each line corresponds to a diffusion free path of the particle according to Section 41 .

The projection of a diffusion path on to a plane can be shown to reduce it on the average in the ratio $\pi / 4$, since it may point in any direction. Thus if $l_{\delta}$ denotes the length of the mean diffusion path of the particles making an angle $\theta$ with a line at right angles to the plane of observation, and $n_{0}$ particles pass through each point per second, the length of the projection of these paths on to the plane is equal to

$$
\begin{aligned}
\int_{0}^{\pi} 2 \pi l_{\delta} \sin \theta \cdot l_{\delta} \cdot d \theta \cdot \frac{n_{0}}{4 \pi l_{\delta}^{2}} \cdot l_{\delta} \sin \theta=\frac{l_{\delta} n_{0}}{2} \int_{0}^{\pi} \sin ^{2} \theta \cdot d \theta \\
=\frac{l_{\delta} n_{0}}{2}[\sin \theta \cdot \cos \theta]_{0}^{\pi}+\frac{\pi n_{0}}{2} l_{\delta}-\frac{l_{\delta} n_{0}}{2} \int_{0}^{\pi} \sin ^{2} \theta \cdot d \theta \\
=\frac{\pi n_{0}}{2} l_{\delta}-\frac{l_{\delta} n_{0}}{2} \int_{0}^{\pi} \sin ^{2} \theta \cdot d \theta
\end{aligned}
$$

and thus is equal to $\frac{\pi n_{0}}{4} l_{\delta}$, or $\frac{\pi}{4} l_{\delta}$ for a single path. The average projection $p$ of a diffusion path on to an axis in the plane of observation can now be shown to be given by

$$
p=\frac{4}{n_{0}} \int_{0}^{\frac{\pi}{2}}\left(\frac{\pi}{4} l_{\delta}\right) \cdot d \phi \cdot \frac{n_{0}}{2 \pi\left(\frac{\pi}{4} l_{\delta}\right)} \cdot\left(\frac{\pi}{4} l_{\delta}\right) \sin \phi .
$$

or

$$
p=\frac{l_{\delta}}{2}
$$


where $\phi$ denotes the angle the projection $\frac{\pi}{4} l_{\delta}$ makes with a line at right angles to the foregoing axis.

The projection $p$ is evidently somewhat greater than twice the average amplitude $A_{1}$ of the curve traced out in the plane of observation by the projected motion of a particle when the solution is given a motion at right angles to the axis of projection of $p$. It is evident that the closer the actual path of the particle resembles a number of straight lines joined together at various angles the smaller is this difference. Its magnitude is impossible to determine theoretically, but it is probably safe to say that it is not likely to be greater than 10 per cent, probably it is often much less. Thus as a first approximation we may take $l_{\delta}$ equal to $4 A_{1}$.

It is possible, however, to determine the exact average value of the mean diffusion path $l_{\delta}$ from curves of the nature shown in Fig. 17. The average wave length $\lambda$ of the curve is evidently equal to the average length of the projection of the diffusion path on to an axis in the plane of observation parallel to the direction of the motion given to the solution. The magnitude of this projection, it should be noticed, depends on the magnitude of the motion of the solution. If $n_{m}$ denote the number of maxima in the curve passed over by the particle in one second, and $V_{s}$ the velocity given to the solution, we have the relation

$$
\lambda=\frac{V_{s}}{n_{m}} .
$$

Each of the quantities in the foregoing equation may be measured directly. The length of path $l_{p}$ traced out in the plane of observation in one second may also be obtained by direct measurement from the curve. This length $l_{p}$ is equal to the length of the curve that would be traced out in the plane of observation if the particle passed over 
its diffusion path according to its definition instead of over its actual path. The value of $l_{p} / n_{m}$ is thus equal to the length of the average projection of the diffusion path on to the plane of observation under the conditions of the experiment. Therefore, since the projection of the diffusion path on to an axis parallel to the motion of the fluid in the plane of observation is $\lambda$, it follows from geometrical considerations that the projection of the path on to an axis in the plane of observation at right angles to the motion of the solution is

$$
\sqrt{\left(\frac{l_{p}}{n_{m}}\right)^{2}-\lambda^{2}}
$$

or

$$
\frac{\lambda}{V_{s}} \sqrt{l_{p}^{2}-V_{s}^{2}}
$$

since $n_{m}=V_{s} / \lambda$. This projection is independent of the motion given to the solution, and is therefore equal to $p$ the projection corresponding to the solution at rest. Therefore, since we have previously obtained that $p=l_{\delta} / 2$, we have

$$
l_{\delta}=\frac{2 \lambda}{V_{s}} \sqrt{l_{p}^{2}-V_{s}^{2}} \text {. . . . . . }
$$

Thus we see that it is possible to determine directly the average diffusion path of a colloidal particle as defined in Section 41.

It is possible therefore to calculate the coefficient of diffusion and the total average velocity of such a particle. According to equation (129) the coefficient of diffusion $D_{r}$ of particles $r$ in a substance consisting of particles $e$ is given by

$$
D_{r}=\frac{n_{r} l_{\delta r}}{N_{r}}
$$

where $n_{r}$ denotes the number of particles $r$ crossing a square $\mathrm{cm}$. from one side to the other per second, and $N_{r}$ the con- 


\section{MISCELLANEOUS APPLICATIONS, CONNECTIONS}

centration of the particles. Now according to equation (35)

$$
n_{r}=\frac{V_{t r} N_{r}}{3},
$$

while directly

$$
t_{r}=\frac{l_{\delta r}}{V_{t r}}
$$

where $t_{r}$ denotes the average period of the diffusion path $l_{\delta r}$ of a particle $r$, and $V_{t r}$ its total average velocity. The diffusion equation may therefore be written

$$
D_{r}=\frac{l_{\delta r}^{2}}{3 t_{r}} \cdot \text {. . . . . . }
$$

The period $t_{r}$ is also given by

$$
t_{r}=\frac{1}{n_{m}}=\frac{\lambda}{V_{s}}
$$

and may thus be determined from curves of the nature shown in Fig. 17. Equation (141) may therefore be used to determine the coefficient of diffusion of colloidal particles.

Since I have not previously published the definition of the free diffusion path used, and the method of determining it directly in the case of a colloidal particle, no values of it have yet been determined. But the average values of the amplitudes $A_{1}$ (Fig. 17) of the curve described by a particle in the plane of observation are, however, available, which, we have seen, are approximately equal to $l_{\delta} / 4$. Svedberg has measured the average amplitude $A_{1}$, and average period $t$, of platinum particles in various solvents. Using these values I have calculated the coefficients of diffusion of the particles, which will be found in Table XXII. These values are probably nearer to the truth than could be obtained by direct measurement. The last column in the Table gives the product $D_{\eta}$, where 
$\eta$ denotes the viscosity of the solvent. It will be seen that it is very approximately constant, and the coefficient of diffusion of a particle thus varies inversely as the viscosity of the medium. This is what we would expect to hold in the case of particles considerably larger than a molecule.

\section{TABLE XXII}

\begin{tabular}{|c|c|c|c|c|c|c|c|}
\hline Solvent. & $\begin{array}{c}\text { Radius } \\
\text { in } \\
\text { cms. } \\
10^{5} .\end{array}$ & $\begin{array}{c}\text { Temp. } \\
\text { Cent. }\end{array}$ & $\begin{array}{c}\text { Time } t \\
\text { in } \\
\text { seconds. }\end{array}$ & $\begin{array}{c}\text { Viscos- } \\
\text { ity } \eta\end{array}$ & $\begin{array}{c}4 A_{1} \text { in } \\
\text { cms. } \\
10^{5} .\end{array}$ & $\begin{array}{c}D \times 10^{7} \\
\text { cm. } \\
\text { sec. }\end{array}$ & $D \eta 10^{10}$ \\
\hline Acetone....... & 0.25 & 18 & 0.032 & .0023 & 14.2 & 2.10 & 4.83 \\
E. Acetate..... & 0.25 & 19 & 0.028 & .0046 & 9.4 & 1.05 & 4.83 \\
Amyl acetate. & 0.25 & 18 & 0.026 & .0059 & 8.0 & .82 & 4.84 \\
Water.......... & 0.25 & 20 & 0.013 & .0102 & 4.3 & .47 & 4.80 \\
Propyl alcohol . & 0.25 & 20 & 0.009 & .0226 & 2.4 & .21 & 4.75 \\
\hline
\end{tabular}

An expression for the total average velocity of a colloidal particle is obtained from the two preceding equations giving the period of the diffusion path, and equation (140), which gives

$$
V_{t r}=\frac{l_{\delta r} V_{s}}{\lambda}=2 \sqrt{l_{p}^{2}-V_{s}^{2}} . . . .
$$

Since values of $l_{p}$ are not available this equation cannot yet be used to calculate values of $V_{t r}$. We may, however, use the approximate average value of $4 A_{1}$ for $l_{\delta r}$, which gives to the equation the form

$$
V_{t r}=4 A_{1} \frac{V_{s}}{\lambda}=\frac{4 A_{1}}{t_{r}}
$$

This equation would hold exactly if the actual path of a particle were along a number of straight lines joined together at various angles. It has been obtained by Sverlberg on this supposition, and used to calculate the velocities of particles. For the platinum particles in various 
solvents to which Table XXII refers he obtained the velocities given in Table XXVI. It will be seen that the velocities do not differ much from one another, and therefore do not seem to depend much on the nature of the solvent since the particles have the same diameter. The values obtained are, however, not exact, as explained before.

We have seen in Section 42 and this Section that the coefficient of diffusion depends directly on the total average velocity of a molecule and the nature of its motion. Since this also holds for other quantities, the coefficients may also be expressed in terms of them. This applies to the quantities osmotic pressure and coefficient of mobility, and their relation to the coefficient of diffusion will therefore now be investigated.

\section{The Coefficient of Diffusion in Connection} with Osmotic Pressure and the Coefficient of Molecular Mobility.

Consider a heterogeneous solution of molecules $e$ and $r$, in which case diffusion of molecules from one place to another takes place until the molecules are uniformly distributed. If a semipermeable membrane impervious tosay molecules $r$, were placed at right angles to a stream of diffusing molecules $r$, they would exert a pressure in the direction of their migration upon the membrane. Since the concentration of the molecules $r$ is different on the two sides of the membrane, this pressure is the difference between the osmotic pressures of the molecules acting on the two sides of the membrane. This pressure is therefore the force acting upon the molecules tending to move them to places of lower concentration, which manifests itself as a pressure by reaction on the membrane placed in the path of the molecules to prevent their migration. In the absence of the membrane this force is spent in overcoming 
the viscous friction exerted by the mixture on the migrating molecules. We may therefore look upon each cubic $\mathrm{cm}$. of molecules $r$ as being under a force equal to the difference in the osmotic pressures of the opposite faces of the cubic $\mathrm{cm}$., which force is spent in giving motion to the molecules against the viscous friction of the medium. This idea of looking upon diffusion is mainly due to Nernst.

In general if $\delta_{r}$ denotes the number of molecules diffusing across a square $\mathrm{cm}$. per second we have

$$
\delta_{r}=N_{r} V_{r}^{\prime}
$$

where $N_{r}$ denotes the concentration of the molecules $r$, and $V^{\prime}{ }_{r}$ the velocity with which each molecule $r$ on the average is moving in the direction of decrease of concentration gradient. Now according to the foregoing considerations we may write

$$
V_{r}^{\prime}=M_{r} \frac{d P_{s r}}{d x}
$$

where $M_{r}$ denotes the coefficient of mobility of a molecule, or its velocity under the action of unit force, and $d P_{s r} / d x$ denotes the osmotic pressure gradient. Hence the preceding equation may be written

$$
\delta_{r}=M_{r} \frac{d P_{s r}}{d x} \cdot \text {. . . . . . }
$$

Similarly in the case of the molecules $e$ diffusing in the opposite direction we have

$$
\delta_{e}=M_{e} \frac{d P_{s e}}{d x}
$$

From the preceding equations and equation we have,

$$
\vartheta_{e} M_{e} \frac{d P_{s e}}{d x}=\vartheta_{v} M_{r} \frac{d P_{s r}}{d x}
$$


an equation which expresses the relation between the osmotic pressure gradients of the molecules $r$ and $e$, their coefficients of mobility, and their molecular volumes.

In the case of a dilute solution-say of molecules $r$ in $e$, the osmotic pressure obeys the gas laws, that is we may write

$$
P_{s r}=\frac{R T}{m_{r}} \rho_{r}=\frac{R T}{\frac{m_{r}}{m_{a r}}} N_{r}=\frac{R T}{N} N_{r},
$$

where $m_{r}$ and $m_{a r}$ denote the relative and absolute molecular weights of a molecule $r$, and the ratio $m_{r} / m_{a r}$ is therefore equal to the number $N$ of molecules in a gram molecule which according to Section 3 is equal to $6.2 \times 10^{23}$. Hence

$$
\frac{d P_{s r}}{d x}=\frac{R T}{N} \frac{d N_{r}}{d x}
$$

and equation (143) becomes

$$
\delta_{r}=M_{r} \frac{R T}{N} \frac{d N_{r}}{d x} .
$$

This gives for the coefficient of diffusion

$$
D_{r}=M_{r} \frac{R T}{N} . \quad . \quad . \quad . \quad .
$$

Thus if the value of either of the two quantities $D_{r}$ and $M_{r}$ be known that of the other quantity may be immediately calculated. Table XXIII gives the values of $M$, or the mobility under unit force, of a number of different molecules in different liquid and gaseous media, calculated from the known values of $D$. Table XXIV gives the calculated values of $D$ for some electrically charged molecules whose mobilities per unit force are known. They agree fairly well with the values obtained by experiment. 


\section{TABLE XXIII}

\begin{tabular}{|c|c|c|c|c|c|c|}
\hline \multirow{2}{*}{$\begin{array}{c}\text { Molecules } \\
\text { for which } \\
D \text { is given } \\
\text { in Table } \\
\mathrm{XX} .\end{array}$} & \multirow{2}{*}{$\begin{array}{c}\begin{array}{c}\text { Gaseous } \\
\text { medium } \\
\text { of } \mathrm{CO}_{2} \mathrm{at} \\
760 \mathrm{~cm} .\end{array} \\
\text { pressure } \\
\text { and } \mathrm{O}^{\circ} \mathrm{C} .\end{array}$} & \multirow{2}{*}{ 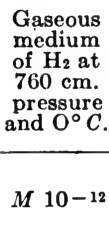 } & \multicolumn{4}{|c|}{ Liquid medium of water. } \\
\hline & & & Molecules. & $\begin{array}{l}\text { Temp. } \\
\text { C. }\end{array}$ & $D \frac{n .^{2}}{\text { day. }}$ & $M 10^{-9}$. \\
\hline $\mathrm{H}_{2} \mathrm{O}$ & 3.6 & 18.8 & Hydrogen. & 10 & 3.75 & 1.14 \\
\hline $\mathrm{CH}_{4} \mathrm{O}$ & 2.4 & 13.7 & $\left\{\begin{array}{c}\text { Nitrous } \\
\text { oxide. }\end{array}\right.$ & 14 & .63 & .19 \\
\hline $\begin{array}{c}\mathrm{C}_{6} \mathrm{H}_{6} \\
\mathrm{C}_{9} \mathrm{H}_{18} \mathrm{O}_{2}\end{array}$ & $\begin{array}{r}1.99 \\
.83\end{array}$ & $\begin{array}{l}8.02 \\
4.70\end{array}$ & $\left\{\begin{array}{r}\text { Cane } \\
\text { Sugar. }\end{array}\right.$ & 12 & .284 & .087 \\
\hline
\end{tabular}

TABLE XXIV

\begin{tabular}{|c|c|c|c|c|c|c|}
\hline $\begin{array}{c}\text { Nature of } \\
\text { gas in } \\
\text { which the } \\
\text { ions are } \\
\text { produced. }\end{array}$ & $\begin{array}{c}\text { values of } M \text { for } \\
\text { tand } \\
\text { calculated from } \\
\text { experimental } \\
\text { results obtained } \\
\text { by Wellisch.* }\end{array}$ & $\begin{array}{c}\text { Values of } D \text { for } \\
\text { and } \\
\text { calculated by } \\
\text { means of equa- } \\
\text { tion (147). }\end{array}$ & $\begin{array}{c}\text { Values of } D \\
\text { obtained di- } \\
\text { rectly by ex- } \\
\text { periment by } \\
\text { Townsend. } \dagger\end{array}$ \\
\cline { 2 - 6 } & $M-10^{12}$ & $M+10^{12}$ & $D-$. & $D+$. & $D-$ & $D+$. \\
\hline $\mathrm{H}_{2}$ & 5.13 & 4.32 & .200 & .169 & $.19 C$ & .123 \\
$\mathrm{O}_{2}$ & 1.16 & .88 & .0453 & .0343 & .0396 & .025 \\
$\mathrm{CO}_{2}$ & .55 & .52 & .0214 & .0201 & .026 & .023 \\
\hline
\end{tabular}

* Phil. Trans.; A, Vol. CXCIII, p. 129 (1900)

†Ibid., A, Vol. CCIX, «. 269 (1909).

On equating the coefficient of diffusion given by equation (146) with that given by equation (129), we have

$$
\frac{n_{r} l_{\delta r}}{N_{r}}=M_{r} \frac{R T}{N} .
$$

Since $n_{r}=\frac{V_{t r} N_{r}}{3}\left(\right.$ Section 18), and $V_{t r} t_{r}=l_{\delta r}$, where $t_{r}$ denotes 
the time the molecule passes over the path $l_{\delta r}$ with the velocity $V_{t r}$ (Section 17), the foregoing equation may be written

$$
\frac{l_{\delta r}^{2}}{t_{r}}=3 M_{r} \frac{R T}{N} . \quad . \quad . \quad . \quad . .
$$

This equation may be used to calculate the mobility under unit force of particles of a size that undergo Brownian - motion. The values of $l_{\delta r}$ and $t_{r}$, we have seen in the previous Section, can be determined by direct observation. Table XXV gives the mobility under unit force of particles

\section{TABLE XXV}

Platinum particles of average radius $.25 \times 10^{-5} \mathrm{Cm}$.

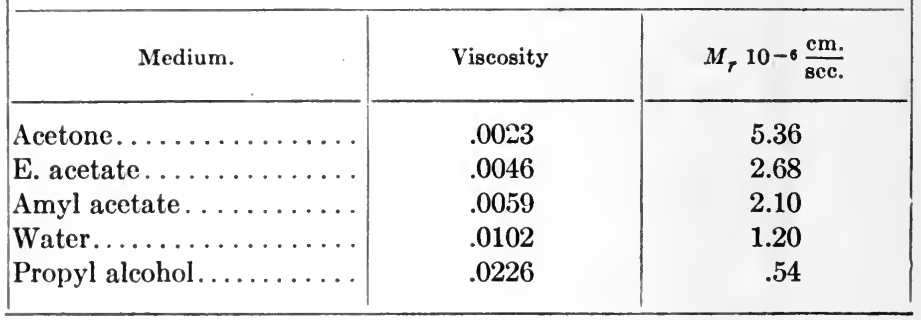

suspended in different kinds of solutions calculated from data contained in Table XXII, using for $l_{\delta r}$ the approximate value $4 A_{1}$.

An expression may be found for the force $F$ on a colloidal particle moving with the observed velocity $V_{c}$ under unit electric field. This force cannot be calculated from electrical data without the introduction of assumptions. A colloidal particle as a whole is not electrically charged, but is supposed to be surrounded by two parallel layers of electricity of equal magnitude but of opposite sign. These layers get distorted when an electric field is applied to the solution, and the tendency of the particle to move so as to 
readjust this tends to give it a continual motion. The force $F$ acting on the particle is immediately given by

$$
V_{c}=F M_{r}
$$

which may be written

$$
F=V_{c} \frac{3 R T t_{r}}{N l_{\delta r}^{2}}
$$

by means of equation (148). Each quantity on the righthand side of this equation may be determined directly and hence $F$ calculated.

Experiment shows that the observed velocity $V_{c}$ of a colloidal particle varies inversely as the viscosity of the solution, or as $1 / \eta$, but is independent of the mass of the particle. Hence for different solvents the force $F$ is inversely proportional to $\eta$, and proportional to $t_{r} / l_{\delta r}^{2}$, or inversely proportional to the mobility, according to equation (148). Since the mobility is proportional to the coefficient of diffusion $D$ according to equation (147), and $D \eta$ is constant according to Table XXII, it follows that the force acting on a colloidal particle in a solution under unit electric field is approximately independent of all conditions at constant temperature.

It will be of interest to determine the absolute value of $F$ in a special case. Thus Bredig found that $V_{c}$ in the case of platinum particles suspended in water had a value of about $.00025 \mathrm{~cm}$. per second for an electric field of one volt per $\mathrm{cm}$. According to Table XXII for platinum particles of radius $.25 \times 10^{-5} \mathrm{~cm}$. suspended in water, $t_{r}=$ .013 sec., $4 A_{1}=.000043 \mathrm{~cm}$. corresponding to $T=293^{\circ}$, while $R=8.315 \times 10^{7}$, and $N=6.2 \times 10^{23}$. Assuming that $4 A_{1}=l_{\delta r}$, which holds approximately, we obtain that

$$
F=2 \times 10^{-10} \text { dyne }
$$


for a platinum particle under the foregoing conditions. This is the force that would act on the particle if it possessed an electric charge equal approximately to $100 e$.

The formulæ for the diffusion and viscosity given in Sections 42 and 35 involve the quantities $n_{r}$ and $n_{e}$, the number of molecules $r$ and $e$ crossing respectively a square $\mathrm{cm}$. from one side to the other per second. These quantities may be expressed in terms of quantities which have not been defined previously.

\section{Partial Intrinsic Pressures.*}

In connection with the intrinsic pressures of a mixture it will be convenient to introduce the quantity partial intrinsic pressure, whereby expressions for various quantities are furnished which are of theoretical interest and often of practical use. Let us consider a mixture of molecules $r$ and $e$ cut into two parts by an imaginary plane $a b$ as shown in Fig. 7. Let $P_{n r^{2}}$ denote the attraction which the molecules $r$ in the portion $B$ exert on the molecules $r$ in a cylinder of unit cross-section and infinite length standing in the portion $A$ with one of its bases on the plane $a b$, and let $P_{n e z}$ have a similar meaning in reference to the molecules $e$. Let $P_{\text {nre }}$ denote the attraction of the molecules $r$ in the portion $B$ on the molecules $e$ in the cylinder, which is also equal to the attraction of the molecules $e$ in this portion on the molecules $r$ in the cylinder. The intrinsic pressure of the mixture in the plane $a b$, which is the sum of the foregoing forces of attraction, is therefore given by

$$
P_{n}=P_{n r^{2}}+2 P_{n r e}+P_{n e 2}, \quad . \quad . \quad .
$$

where the quantities on the right-hand side of the equation may be called the partial intrinsic pressures of the mixture.

$$
\text { * Matter published for the first time. }
$$


The molecules $r$ in one of the portions of the mixture are thus attracted by the other porsion as a whole by a force which gives rise to the pressure $P_{n r^{2}}+P_{n r e}$ per $\mathrm{cm} .^{2}$ in the plane $a b$. This force acting on the molecules $r$ can be sustained only by their expansion pressure, in other words, no part of the force can be sustained by the expansion pressure of the molecules $e$ in the plane $a b$. For suppose the part $I_{r}$ of the intrinsic pressure $P_{n r 2}+P_{n r e}$ is sustained by the part $X_{e}$ of the expansion pressure of the molecules $e$. The molecules $r$ may then be said to be under a component force $I_{r}$, and the molecules $e$ under a component force $X_{e}$, acting in the opposite directions, which react upon each other through the medium of the interaction of the molecules $r$ and $e$ of the mixture. But if two forces act upon two different sets of molecules in this way they would tend to be set in motion in opposite directions, and would react upon each other only through the viscous resistance they would exert upon each other. The two sets of molecules would therefore gradually get separated through diffusing through each other. But this does not take place since the mixture is in equilibrium. The forces in question are therefore equal to zero, or the intrinsic pressure $P_{n r 2}+P_{n r e}$ associated with the molecules $r$ is sustained by a part of their expansion pressurc.

A quantity similar to the partial intrinsic pressure may be defined in connection with the external pressure of a mixture. This pressure is exerted in part by each set of molecules, and in the case of a mixture of molecules $e$ and $r$ the total external pressure $p$ may therefore be written

$$
p=p_{r}+p_{e}, \quad \text {. . . . . . }
$$

where $p_{r}$ and $p_{e}$ denote respectively the partial external pressures of the molecules $r$ and $e$. It is obvious that the partial external pressure $p_{r}$ represents a part of the expan- 


\section{MisCELlaNEOUS APPLICATIONS, CONNECTIONS}

sion pressure of the molecules $r$, and a similar remark applies to the partial external pressure of the molecules $e$. It follows then from Section 20 that if $n_{r}$ denote the number of molecules $r$ crossing a square $\mathrm{cm}$. in the mixture from one side to the other, and $n_{e}$ has a similar meaning with respect to the molecules $e$, we have

$$
\begin{aligned}
p_{r}+P_{n r^{2}}+P_{n r e}=n_{r} \frac{v_{r}}{v_{r}-b^{\prime}{ }_{r}} & \frac{A_{r}}{2} \\
& =2.543 \times 10^{-20} n_{r} \frac{v_{r}}{v_{r}-b^{\prime}{ }_{r}} \sqrt{T m_{r}},
\end{aligned}
$$

and

$$
\begin{aligned}
p_{e}+P_{n e^{2}}+P_{n r e}=n_{e} \frac{v_{e}}{v_{e}-b^{\prime}} & \frac{A_{e}}{2} \\
& =2.543 \times 10^{-20} n_{e} \frac{v_{e}}{v_{e}-b_{e}^{\prime}} \sqrt{T m_{e}} .
\end{aligned}
$$

The partial intrinsic pressures cannot yet be expressed similarly as the quantity $P_{n}$ in terms of other quantities which can be directly measured. Approximate numerical values may, however, be obtained from the simultaneous equations (150), (152), and (153), by neglecting the partial external pressures in comparison with the partial intrinsic pressures, which may usually be done, and calculating the quantities $n_{r}, n_{e}, b_{r}^{\prime}, b^{\prime}{ }_{e}$, and $P_{n}$, by the method of Sections 21 and 29.

Approximate values may also be obtained by the following method. In the case of a pure substance the intrinsic pressure according to Section 26 may as a first approximation be taken proportional to the square of the density $\rho$ of the substance. Applying this result to the kind of mixture under consideration the quantities $P_{n r^{2}}$ and $P_{n e z}$ may evidently be written

and

$$
P_{n r 2}=B^{2}{ }_{r}{ }^{2}{ }_{r}, \text {. . . . }
$$

$$
P_{n e^{2}}=B^{2}{ }_{e}^{2}{ }^{2}, \quad \text {. . . . }
$$


where $\rho_{r}$ and $\rho_{e}$ denote the partial densities of the molecules $r$ and $e$ in the mixture, and $B_{r}$ and $B_{e}$ denote approximate constants. As a first approximation the quantity $P_{n \text { re }}$ is then given by

$$
P_{n r e}=B_{e} B_{r} \rho_{e} \rho_{r}, \quad \text {. . . . . }
$$

The values of the quantities $B_{r}$ and $B_{e}$ may be obtained from the internal heats of evaporation $L_{r}$ and $L_{e}$ of gram molecules of the substances in the pure state. Thus according to Section 21 we have

$$
L_{r}=\int P_{n} \cdot d v=-\int_{\rho_{1}}^{\rho_{2}} P_{n} \frac{m_{r}}{\rho^{2}} \cdot d \rho=m_{r} B_{r}^{2}\left(\rho_{1}-\rho_{2}\right),
$$

where $\rho_{1}$ and $\rho_{2}$ denote the densities of the pure substance $r$ in the liquid and vaporous states, and a similar equation may be obtained for $L_{e}$. These equations express $B_{r}$ and $B_{e}$ in terms of $L_{r}$ and $L_{e}$.

The values for the partial intrinsic pressures obtained by this or the preceding method may be tested by substituting them in equation (150), and determining $P_{n}$ (the intrinsic pressure of the mixture as a whole) by the method described in Section 21. If an agreement is obtained we may be fairly sure that the values of the partial intrinsic pressures obtained are very approximately correct.

If the latter method is used to determine the partial intrinsic pressures, we may use equations (152) and (153) to determine $n_{e}$ and $n_{r}$. We are thus furnished with another method of determining the latter quantities, which is comparatively simpler to use than that described in Section 29.

The latter method of obtaining the partial intrinsic pressures may evidently also be used to find the intrinsic pressure of a pure substance.

In the case of a mixture of more substances than two the corresponding partial intrinsic pressures will not bc 
difficult to define, and may be obtained in a similar way as described.

The partial intrinsic pressures of a heterogeneous mixture may also be connected with its osmotic pressures. Before discussing the connection it will be necessary to consider some general conditions of equilibrium.

47. Conditions of the Equilibrium of a Heterogeneous Mixture such as Two Phases in Contact.

The relative concentration of the constituents of two phases of a mixture is usually different and gradually changes from one to the other in the transition layer which exists at the boundary of the phases. In the case of a mixture of molecules $r$ and $e$, for example, equations (152) and (153) must hold for both phases and the transition layer, and are therefore two of the equations of equilibrium.

The partial external pressures $p_{r}$ and $p_{e}$ in these equations must have the same values everywhere, otherwise diffusion from one portion to the other would take place, and the system would not be in equilibrium. This follows at once from considering two layers of liquid in which the partial external pressures have the values $p_{r}, p_{e}$, and $p_{r}^{\prime}$, $p_{e}^{\prime}$, respectively. Since the total pressure is the same everywhere

$$
p_{e}+p_{r}=p_{e}^{\prime}+p_{r}^{\prime}
$$

and hence

$$
p_{e}-p^{\prime}{ }_{e}=p^{\prime}{ }_{r}-p_{r}
$$

From the latter equation it follows that there is an excess of pressure of the molecules $r$ in one direction which is balanced by an excess of pressure of the molecules $e$ in the opposite direction. But this would give rise to a diffusion of the two sets of molecules through each other, reasoning 
along the same lines as in the previous Section, and thus disturb the equilibrium. Hence we must have

$$
p_{r}=p_{r}^{\prime} \text { and } p_{e}=p_{e .}^{\prime} \quad . \quad . \quad .
$$

If $n_{r}$ denotes the number of molecules $r$ crossing a square cm. from one side to the other, $n_{r}^{\prime}$ the number crossing in the opposite direction, and $n_{e}$ and $n^{\prime}{ }_{e}$ have similar meanings with respect to the molecules $e$, we must also have

and

$$
\left.\begin{array}{l}
n_{e}^{\prime}=n_{e} \\
n_{r}^{\prime}=n_{r}
\end{array}\right\}, \cdot \cdot \cdot \cdot
$$

otherwise diffusion from one place to the other would take place.

The foregoing equations are of special interest in connection with the transition layer, since this has molecular concentration gradients the tendency of which is to give to $n_{r}$, the molecules crossing a square $\mathrm{cm}$. in one direction, a value different from $n_{r}^{\prime}$, the number crossing in the opposite direction, and to give also different values to $n_{e}$ and $n_{e}^{\prime}$. But there evidently exists a force in the layer at right angles to it acting in the direction of increase of density, due to the existence of molecular forces of attraction. This force is equal to the intrinsic pressure gradient. It has the effect of decreasing the velocity of the molecules moving in the direction of decrease of density and increasing the velocity of the molecules moving in the opposite direction. The magnitude of this modification of the velocity of a molecule depends on its nature, since the various molecules do not possess the same forces of attraction. Thus the effect of the intrinsic pressure gradient on the number of molecules crossing a square $\mathrm{cm}$. from one side to the other per second is opposite to that of the concentration gradient, and therefore $n_{r}$ and $n_{r}^{\prime}$, and $n_{e}$ and $n_{e}^{\prime}$, may be rendered equal, as is 


\section{MISCELLANEOUS APPLICATIONS, CONNECTIONS}

the case in practice. Besides, since the effect of the intrinsic pressure gradient on a set of molecules depends on their nature, the concentration gradient would not neoessarily be the same for each set of molecules corresponding to the equilibrium conditions (152) and (153). Thus we see why the relative concentration of the ingredients of a mixture differs in the vaporous phase from that in the liquid phase. It will also be evident that, since the attraction of a molecule increases with its mass, the relative concentration of the heavier molecule is likely to be smaller in the vaporous phase than in the liquid phase. This is exemplified in practice, though it need not, and does not, hold in every case.

Similar conditions apply to a mixture of more than two constituents.

\section{Osmotic Pressure expressed in Terms of} the Kinetic Properties of Molecules.*

If the different portions of a mixture of substances are not in equilibrium with each other, which would manifest itself by a redistribution of the ingredients by diffusion, the equations of equilibrium given in the foregoing Section will not hold. Evidently equations (157) will not hold since the vapor pressure of the molecules $r$ depends on their concentrations. A force equal to $\frac{\delta p_{r}}{\delta x}$, where $x$ is measured along a line of decrease of concentration, will therefore act on each cubic cm. of molecules $r$. Equation (152) may hold at certain parts of the mixture, though in general this would not be the case. There is therefore an additional force equal to

$$
\begin{gathered}
\pm\left\{\frac{\delta}{\delta x}\left(\frac{A_{r}}{2} \frac{v_{r} n_{r}}{v_{r}-b_{r}^{\prime}}\right)-\frac{\delta}{\delta x}\left(P_{n r 2}+P_{n e r}-p_{r}\right)\right\} \\
* \text { Matter published for the first time. }
\end{gathered}
$$


acting on the molecules $r$ per cubic $\mathrm{cm}$. The total force $F$, acting on the molecules $r$ per cubic $\mathrm{cm}$. is therefore given by

$$
\begin{aligned}
F_{r}= \pm\left\{\frac{A_{r}}{2} \frac{v_{r}}{v_{r}-b_{r}^{\prime}} \frac{\delta n_{r}}{\delta x}+\right. & \frac{A_{r}}{2} \frac{n_{r} v_{r}}{\left(v_{r}-b_{r}^{\prime}\right)^{2}} \frac{\delta b_{r}^{\prime}}{\delta x} \\
& +\frac{A_{r} n_{r}}{2}\left(\frac{1}{v_{r}-b^{\prime}{ }_{r}}-\frac{v_{r}}{\left(v_{r}-b^{\prime}\right)^{2}}\right) \frac{\delta v_{r}}{\delta x} \\
& \left.-\frac{\delta}{\delta x}\left(P_{n r^{2}}+P_{n e r}-p_{r}\right)\right\}+\frac{\delta p_{r}}{\delta x} .
\end{aligned}
$$

This force is equal to the osmotic pressure acting on the molecules $r$. It gives rise to a diffusion of molecules equal to $n_{r}-n_{r}^{\prime}$; and equations (158) therefore also do not hold. It is likely that the upper sign of the first term of the righthand side of the foregoing equation always holds in practice.

Similarly the force $F_{r}$ acting on the molecules $e$ per cubic $\mathrm{cm}$. giving rise to a diffusion of molecules $e$ in the opposite direction to the molecules $r$, is given by

$$
\begin{aligned}
F_{e}= \pm\left\{\frac{A_{e}}{2} \frac{v_{e}}{v_{e}-b_{e}^{\prime}} \frac{\delta n_{e}}{\delta x}+\right. & \frac{A_{e}}{2} \frac{n_{e} v_{e}}{\left(v_{e}-b_{e}^{\prime}\right)^{2}} \frac{\delta b^{\prime} e_{e}}{\delta x} \\
& +\frac{A_{e} n_{e}}{2}\left(\frac{1}{v_{e}-b^{\prime}{ }_{e}}-\frac{v_{e}}{\left(v_{e}-b^{\prime}\right)^{2}}\right) \frac{\delta v_{e}}{\delta x} \\
& \left.-\frac{\delta}{\delta x}\left(P_{n e}+P_{n e r}-p_{e}\right)\right\}+\frac{\delta p_{e}}{\delta x} .
\end{aligned}
$$

Since $n_{r}$ depends on the motion of translation of the molecules and molecular attraction, the foregoing equations express osmotic pressure in terms of molecular motion, molecular attraction, and the apparent molecular volume. It will be evident on reflection that osmotic pressure must arise through these properties of matter, and the equations are therefore fundamental in character. They are, however, of little use in practice to calculate osmotic pressure, since we have no means yet of determining experimentally how 
the quantities $n_{r}, b_{r}^{\prime}, P_{n r^{2}}$, and $P_{n e r}$ vary in a heterogeneous mixture from one part to another.

Osmotic pressure as exhibited in connection with semipermeable membranes will be discussed in connection with another subject.

Since the quantities $n_{e}$ and $n_{r}$ in the foregoing and other formulæ depend on the total average velocities of the molecules $e$ and $r$, it will be of importance to see if some direct information can be obtained about the values of these velocities.

49. A Method of Obtaining the Velocity of Translation of Particles undergoing Brownian Motion.

The path of such a particle we have seen in Section 44, is zigzag in shape. The average velocity of translation of the particle may therefore, according to this Section, be determined from observations of the average period $t$ of the free path $l_{\delta}$ by means of the equation

$$
V_{\imath}=\frac{l_{\delta}}{t} \text {. . . . . . . }
$$

Table XXVI contains the approximate velocities of platinum particles in different solvents determined in this way from observations of the amplitudes $A_{1}$ contained in Table XXII made by Svedberg and described in Section 44, which we have seen are approximately equal to $l_{\delta} / 4$. It is usually supposed that the average velocity of a colloidal particle is the same it would have in the perfectly gaseous state. But on calculating the average velocity $V_{a}^{\prime}$ under these conditions, which is given by $V_{a}=\cdot 922 \mathrm{~V}$ and equation (8), it is found that it does not agree with that observed, as will appear from Table XXVI, which gives the velocities of platinum particles obtained in these two ways. Now this is what we would expect from the results of Sections 16 and 
29. But it has yet to be shown why the actual velocities might be smaller in the case of a colloidal particle than corresponding to the gaseous state, when in the case of a single molecule in a liquid we have seen it has a greater value. It appeared from Section 44 that a colloidal particle is continually under the influence of the surrounding molecules in varying degrees, and continually bombarded from all sides by molecules, whose resultant effect is not zero

\section{T'ABLE XXVI}

\begin{tabular}{|c|c|c|}
\hline \multicolumn{3}{|c|}{$\begin{array}{l}\text { Platinum particles of average radius } .25 \times 10^{-5} \mathrm{CM} \text {. } \\
\qquad V_{a} \text { For GaSeOUS State }=8.72 \frac{\mathrm{CM} .}{\mathrm{sec} .}\end{array}$} \\
\hline Medium. & Viscosity. & $V_{t} 10^{5} \frac{\mathrm{cm} .}{\mathrm{sec} .}$ \\
\hline 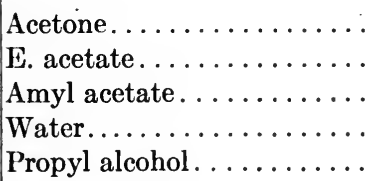 & $\begin{array}{l}.0023 \\
.0046 \\
.0059 \\
.0102 \\
.0226\end{array}$ & $\begin{array}{l}444 \\
336 \\
308 \\
324 \\
266\end{array}$ \\
\hline
\end{tabular}

but continually changes in direction and magnitude, giving the particle the well-known zigzag motion. Therefore when the particle begins to move in any new direction its motion is at once impeded by the viscous friction of the surrounding medium, due to the particle being constantly more or less under the influence of the surrounding molecules. Hence if the initial velocity is that corresponding to the gaseous state, or greater, it may be slowed down to a much smaller value before the particle receives a new impetus, and the total average velocity is therefore not likely to be the same as in the gaseous state, but may be much less. Section 60 also deals with this point. 
It is often assumed that the velocity of a colloidal particle obtained from observations of its path does not represent the true velocity, since the amplitude of the oscillatory motion may occasionally be so small that it cannot be observed by means of the ultra-microscope, and what in truth is a wavy curve of small amplitude and wave lengths would be taken for a smooth line. This is no doubt partly true. But it is highly improbable that only $\frac{1}{10}$ of the path of the particle should be possible of being observed by the ultra-microscope. Otherwise the oscillatory motion of the particle would largely correspond to amplitudes whose magnitude could not be determined by the microscope, on which are impressed amplitudes of very much greater magnitude. This does not seem to be in harmony with the distribution of the amplitudes according to Clausius' law given in Section 31. For this reason, and that the particle must always be subject to viscous friction in its motion, we conclude that the velocity need not be that corresponding to the gaseous state, but is probably much less.

Some experiments by Exner, who showed that particles of diameters $1.3 \mu, .9 \mu$, and $.4 \mu$, have the velocities 2.7, 3.3 , and 3.8 , respectively, point to the same conclusion. For the masses of the particles are proportional to the cubes of their diameters, and their velocities in the gaseous state therefore proportional to the $\frac{3}{2}$ power of their diameters. But this is evidently not realized.

If $V_{1}$ denote the velocity of a particle at any instant in passing over the distance $\delta l$, and $R$ the viscous resistance of the medium, we have

$$
R \cdot \delta l=\delta \cdot\left(\frac{1}{2} m_{a} V^{2}{ }_{1}\right)=m_{a} V_{1} \cdot \delta V_{1},
$$

where $m_{a}$ denotes the mass of the particle. The resistance $R$ is proportional to $V_{1}$, or equal to $K_{2} V_{1}$, where $K_{1}$ denotes 
a constant, and hence the preceding equation on substituting for $R$ and integrating gives

$$
l=\frac{m_{a}}{K_{2}}\left(V_{\imath}-V_{e}\right)
$$

where $V_{i}$ denotes the initial velocity of the particle when it changes its direction, $V_{e}$ the velocity when it is about to receive a new impetus, and $l$ the length of path between the corresponding points.

It follows therefore that the rate of change of $V_{1}$, or the negative acceleration, is constant when the particle is not propelled by the molecules of the medium.

The value of $K_{2}$ is probably very different from the value of the coefficient of mobility referring to the average velocity of a particle parallel to a given direction considered over a time that gives a constant velocity under the action of an external force. The latter constant has been considered in Section 45 and is the factor of $V_{c}$ in equation (149).

The result of this Section is of interest in connection with the osmotic pressure of dilute solutions considered in the next Section.

50. The Osmotic Pressure of a Dilute Solution of Molecules, and their Velocity of Translation.*

Van't Hoff has shown that it follows from thermodynamics that the osmotic pressure of a dilute solution obeys the gas equation, that is, we may write

$$
P_{s T}=\frac{R T \rho}{m}=\frac{R T N_{r}}{N}, \quad . \quad . \quad .
$$

where $N$ denotes the number of molecules in a gram mole* Not previously published. 


\section{MisCELLANEOUS APPLICATIONS, CONNECTIONS}

cule of a substance, and $N_{r}$ the concentration of the solute molecules $r$ in the solvent $e$. This result has given rise to the erroneous statement often made that the velocity of translation of each solute molecule is the same as that it would have in the perfectly gaseous state. It is obvious that if this be true for the solute molecules it must also be true for the molecules of a pure liquid. But it can be shown in many ways that this deduction cannot hold, though Van't Hoff's law be true.

If the velocity of translation of a molecule is always the same as in the gaseous state the expansion pressure of a substance would be given by the expression $\frac{R T}{v-b}$, according to equation (40) in Section 20. Now the external pressure of a substance is not equal to this expression, and therefore forces must exist between the molecules which give rise to an intrinsic pressure which, together with the external pressure, balances the expansion pressure, as expressed by equation (45). But if the molecules exert forces of attraction and repulsion upon each other these will have the effect of changing the velocity of a molecule from what it would have in the gaseous state as shown in Section 17. According to Sections 25 and 29 this total average velocity of a molecule in a liquid is many times what it would have in the gaseous state. Another method used in Section 34 gave the same result.

It can also be shown that this result is a consequence of Section 48, which gives us besides other information. Let us suppose that the vapor pressure of the solute $r$ of a solution is zero, as is the case of a salt dissolved in water. Since the molecules of the solute in the solution according to the definition of a dilute solution are so far apart that their influence upon each other is practically zero, we have $P_{n r^{2}}$ $=0$. According to Van't Hoff's law $F_{r}=\frac{\delta P_{s r}}{d x}=\frac{R T}{N} \frac{\delta N_{r}}{d x}$ along 
a concentration gradient in the mixture. Equation (159) therefore in this case becomes

$$
\begin{aligned}
\frac{R T}{N} \frac{\delta N_{r}}{\delta x}=\frac{A_{r}}{2} \frac{v_{r}}{v_{r}-b^{\prime}} \frac{\delta n_{r}}{\delta x}+\frac{A_{r}}{2} \frac{n_{r} v_{r}}{\left(v_{r}-b_{r}^{\prime}\right)^{2}} \frac{\delta b_{r}^{\prime}}{\delta x} \\
\quad+\frac{A_{r} n_{r}}{2}\left(\frac{1}{v_{r}-b_{r}^{\prime}}-\frac{v_{r}}{\left(v_{r}-b_{r}^{\prime}\right)^{2}}\right) \frac{\delta v_{r}}{\delta x}+\frac{\delta P_{n r e}}{\delta x} .
\end{aligned}
$$

Now if the velocity of translation of a molecule $r$ were the same as in the gaseous state $n_{r}$ would be given by equation (21) on adding suffixes $r$ to the symbols, and $\frac{\delta n_{r}}{\delta N_{r}}$ would be given by equations (162) and (20), in which case

$$
\frac{\delta n_{r}}{\delta N_{r}}=\frac{2 R T}{N A_{r}} .
$$

Therefore on dividing equation (163) by $\frac{\delta N_{r}}{\delta x}$, and taking into account that $N_{r} v_{r} m_{a r}=m_{r}$, it may be written

$$
\begin{aligned}
\frac{R T}{N_{J}}=\frac{v_{r}}{v_{r}-b_{r}^{\prime}}, \frac{R T}{N}+\frac{A_{r}}{2} \frac{n_{r} v_{r}}{\left(v_{r}-b_{r}^{\prime}\right)^{2}} \frac{\delta b_{r}^{\prime}}{\delta N_{r}} & \\
& \quad \frac{\delta P_{n r e}}{\delta N_{r}}-\frac{R T v_{r}}{N_{r}}\left(\frac{1}{v_{r}-b_{r}^{\prime}}-\frac{v_{r}}{\left(v_{r}-b_{r}^{\prime}\right)^{2}}\right) .
\end{aligned}
$$

But the right-hand side of this equation is not identically equal to the left-hand side, for $\frac{v_{r}-b_{r}^{\prime}}{v_{r}}$.is not approximately equal to unity, since the value of $b_{r}{ }^{\prime}$ according to Section 20 is determined by the volume of the molecules $e$ as well as that of the molecules $r$ in a gram molecule of molecules $r$, and the value of $b^{\prime}{ }_{r}$ is therefore not small in comparison with $v_{r}$; for the same reason $\frac{\delta b_{r}^{\prime}}{\delta N_{r}}$ is not zero; and $\frac{\delta P_{n r e}}{\delta N_{r}}$ is not zero since molecular forces exist. Thus 
in general the velocity of translation of the molecules $r$ would not be equal to that in the gaseous state.

We may also note the following considerations in this connection. If we suppose that the velocity of the solute molecules in a dilute solution is the same as that which they would have in the perfectly gaseous state their external pressure is given by $\frac{R T}{v_{r}-b_{r}^{\prime}}$. Now the apparent volume $b^{\prime}{ }_{r}$ of the molecules of the mixture, or the apparent volume which the molecules $e$ and $r$ appear to possess in obstructing the motion of the molecules $r$, is not small in comparison with $v_{r}$. Its value is approximately equal to $v_{r} b_{e} / v_{e}$, where $b_{\epsilon}$ refers to the solvent $e$ in the pure state. According to the above supposition Van der Waals' equation holds, and if applied to the solvent $e$ in the pure state we have

$$
p+P_{n}=\frac{R T}{v_{e}-b_{e}}
$$

(Section 26) where $P_{n}$, the intrinsic pressure, is large in comparison with the external pressure $p$, and large (Section 21 ) in comparison with the pressure $p^{\prime}$ the substance would have if it behaved as a perfect gas. It follows therefore from the equation that $b_{e} / v_{e}$ is not small in comparison with unity. Hence on substituting $v_{r} b_{\epsilon} / v_{e}$ for $b^{\prime}{ }_{r}$ in $\frac{R T}{v_{r}-b_{r}^{\prime}}$ it becomes $\frac{R T^{\cdot}}{v_{r}\left(1-b_{e} / v_{e}\right)}$, and thus the pressure exerted by the solute molecules is many times that which they would have in the perfectly gaseous state. This pressure is in part balanced by the intrinsic pressure of the mixture. We cannot, therefore, with any show of reason, say that because the osmotic pressure of the solute molecules obeys the gas laws their velocity of translation is the same as in the gaseous state. 
The values of $n_{r}$ and $P_{n r e}$ of a dilute solution are evidently proportional to $N_{r}$, while $b^{\prime}{ }_{r}$ is proportional to $v_{r}$, and we may therefore write

$$
n_{r}=a_{1} N_{r}, b^{\prime}{ }_{r}=a_{2} v_{r}, \text { and } P_{n r e}=a_{3} N_{r},
$$

where $a_{1}, a_{2}$, and $a_{3}$ are constants. Equation (163) may therefore be written

$$
a_{1}=\frac{2 R T\left(1-a_{2}\right)}{N A_{r}}-\frac{2\left(1-a_{2}\right) a_{3}}{A_{r}}, . . .
$$

on taking into account that

$$
N_{r} v_{r} m_{a r}=m_{r}
$$

which gives a relation between the foregoing constants.

A method of determining directly the number of molecules in a gram molecule which is based on equation (162) will now be described.

51. A Direct Determination of $N$, the Number of Molecules in a Gram Molecule.

Perrin* has determined directly the value of $N$ by a method of counting the number of particles in a dilute colloidal solution. The gravitational attraction of the particles tends to deposit them on to the bottom of the vessel containing the solution. The concentration gradient caused thereby gives rise to an osmotic pressure acting in the opposite direction to the gravitational attraction. Equilibrium exists when these forces balance one another, which corresponds to an increase in the concentration of the solution with increase of distance from the surface.

${ }^{*}$ C. R., 147 (1908), p. 530; 147 (1908), p. 594; Ann. Chim. Phys., 8, 18 (1909), pp. 5-174; Bull. Soc. Fr. Phys., 3 (1909), p. 155; Zs.f. Electroch., 15 (1909), p. 269. 
Since the osmotic pressure obeys the gas laws the distribution of the particles is similar to that of the molecules of a gas under the action of gravity. The differential equation of equilibrium is

$$
F \cdot \delta h=\delta P_{s},
$$

where $P_{s}$ denotes the osmotic pressure at a distance $h$ from the surface of the solution, and $F$ the force due to gravity acting on the particles in a cubic $\mathrm{cm}$. of the solution. If $v_{a}$ denote the volume of a particle, $\rho_{a}$ its density, $\rho$ the density of the liquid, we have

$$
F=v_{a}\left(\rho_{a}-\rho\right) g N_{c},
$$

where $N_{c}$ denotes the concentration of the particles. For $P_{s}$ we have

$$
P_{s}=\frac{R T}{N} N_{c}
$$

according to equation (162), and hence the equation of equilibrium becomes

$$
v_{a}\left(\rho_{a}-\rho\right) g N_{c} \cdot \delta h=\frac{R T}{N} \cdot \delta N_{c},
$$

which on integration gives

$$
\left(h_{1}-h_{2}\right) v_{a}\left(\rho_{a}-\rho\right) \frac{g N_{c} N}{R T}=\log \frac{N_{c}^{\prime}}{N_{c}^{\prime \prime},}
$$

where $N^{\prime}{ }_{c}$ and $N^{\prime \prime}{ }_{c}$ denote the concentrations corresponding to the distances $h_{1}$ and $h_{2}$ from the surface of the solution. The values of $N^{\prime}{ }_{c}$ and $N^{\prime \prime}{ }_{c}$ corresponding to given values of $h_{1}$ and $h_{2}$ were found by counting the particles in different planes in a solution placed under the ultra-microscope. Solutions of gamboge and mastic were used.

The densities of the particles in the solutions were determined by two methods. In one method it was taken the same as that of the substance in the undivided state, 
while in the other a known volume of the solution was evaporated and the residue weighed. The two methods gave concordant results.

The volume of a particle was obtained, in one of the three methods used, by counting the number of particles in a given volume of emulsion and, knowing their weight and density, the volume was immediately obtained.

In the second method the emulsion was slightly acidulated, which has the effect of making the particles stick together in little strings which adhere to the vessel's walls. On measuring the length of a string and counting the number of particles in it, their radii could be determined.

The third method depended on an application of Stokes' law to the rate of fall of the particles under the action of gravity.

These methods gave concordant results. Thus in one case the three methods gave for the same particle the diameters $.46 \mu, .455 \mu$, and $.45 \mu$. Particles of mastic and gamboge in a solution thus appear to be spherical in shape, and their motion obeys Stokes' law.

These measurements determine the various quantities in equation (165) except $N$, which is therefore expressed in terms of these quantities. In this way Perrin obtained

$$
N=7.05 \times 10^{23},
$$

which agrees as well as can be expected with the value given in Section 3.

It is very important to notice that this investigation does not depend upon the velocity of translation of the particles being the same as if they were in the perfectly gaseous state. It depends merely upon the osmotic pressure obeying the gas equation, which according to Section 50 may be the case independent of the velocity of translation of the particles. This point is of importance because these 
experiments are occasionally cited as proving, on account of the occurrence of the factor $\frac{R T}{N}$ in equation (165), that the colloidal particles in a solution have the same velocity as they would have in the gaseous state.

\section{A Free Path Formula involving Stokes' Law.}

According to Stokes' law if a spherical particle of radius $r$ is under the action of a force $F$ in a medium of viscosity $\eta$ and density $\rho$, and there is no slipping (Section 34), the velocity $V_{c}$ with which the particle moves is given by

$$
V_{c}=\frac{F}{6 \pi r \eta} .
$$

Since $V_{c}=M F$, where $M$ denotes the coefficient of mobility, the equation may be written

$$
M=\frac{1}{6 \pi r \eta} . \quad . \quad . \quad . \quad .
$$

On substituting this expression for $M$ in equation (148) it becomes*

$$
\frac{l_{\delta}^{2}}{t}=\frac{R T}{N} \frac{1}{2 \pi r \eta} . \quad . \quad . \quad . \quad .
$$

Since the value of $l_{\delta}$ differs little from four times the average amplitude $A_{1}$ of a particle observed in practice (Section 44) the equation should agree approximately with the facts provided Stokes' law holds. If for example the values of $4 A_{1}$ and $t$ (Section 44) observed by Svedberg for platinum particles are substituted in the left-hand side of the equation for $l_{\delta}$ and $t$ it does not agree even approximately with the values of the right-hand side, as is shown in Table XXVII. This may be caused by the values of $4 A_{1}$ differing more * Not previously published. 
from the values of $l_{\delta}$ than is apparent. The values of $4 A_{1}$ are probably otherwise unobjectionable since the coefficients of diffusion of platinum particles in different solvents calculated from them (Section 44) give very approximately $D_{\eta}=$ constant. It is more likely that the motion of platinum particles in a solution obtained by the sparking of platinum electrodes in a solvent does not obey Stokes' law. This is perhaps not surprising since these particles are not likely to be spherical in shape as required by this law, but more likely consist of flakes, since they are produced by portions of the electrodes being torn off through the electrical discharge.

The particles of gamboge used by Perrin in the investigation described in the previous Section are more likely to be spherical in shape since they were prepared by rubbing gamboge in distilled water giving a yellow solution containing particles of various sizes whose corners would more or less be dissolved off. Thus the motion of such particles might obey Stokes' law, as Perrin has directly observed. Values of $l_{\delta}$ and $t$ for such particles are, however, not available to test equation (167).

But whatever the geometrical configuration of the particle the velocity $V_{c}$ is likely to vary inversely as $\eta$, or

$$
V_{c}=F K_{c / \eta} / \eta \text {. . . . . }
$$

where $K_{c}$ is a constant depending on the nature of the particle; and accordingly equation (148), since $V_{c}=M F$, may be written

$$
\frac{l_{\delta}^{2}}{t}=\frac{3 R T}{N} \frac{K_{c}}{\eta}
$$

This equation appears to agree in a general way with the facts. It might be used to calculate $K_{c}$, which has been carried out for platinum particles in Table XXVII, using 
the data contained in Table XXII. The values obtained are practically constant, as we would expect that they should be if the mass of the particles is kept the same.

\section{TABLE XXVII}

\begin{tabular}{|c|c|c|c|}
\hline \multicolumn{4}{|c|}{ Platinum Particles of radius $.25 \times 10^{-5} \mathrm{cms}$. } \\
\hline Solvent. & $10^{7} l_{\delta}^{2} / t$ & $10^{7} \frac{R T}{N} \frac{1}{2 \pi r \eta}$ & $K_{c} 10^{-4}$ \\
\hline Acetone ............. & 6.45 & 10.9 & 3.688 \\
\hline E. acetate ............ & 2.51 & 5.43 & 2.934 \\
\hline Amyl acetate.... & 2.46 & 4.24 & 3.696 \\
\hline Water.......... & 1.42 & 2.45 & 3.693 \\
\hline Propyl alcohol. . . & .640 & 1.11 & 3.681 \\
\hline
\end{tabular}

Some interesting and important extensions of the viscosity, conduction of heat, and diffusion formulæ will now be given which involve an extended meaning of the molecular paths under various conditions. Following these a number of important formulæ related to the foregoing will be developed involving the projection of the motion of a molecule along an axis, instead of the molecular path. By means of these formulæ further information about the motion of a molecule and other of its properties may be obtained. The foregoing developments are new, and have not been published previously.

\subsection{Extended Forms of the Diffusion Equations.}

The most important property of the mean diffusion path of a molecule defined in Section 41 which is contained in its definition is, that the sum of the diffusion paths between two points is equal to the corresponding length of the actual path of the molecule. This property enables us to express the number of diffusion paths $n$ crossing a plane 
one square $\mathrm{cm}$. in area, a quantity which occurs in the diffusion equations, in terms of quantities which can be determined directly or indirectly. We may, however, give other definitions to the diffusion path, which, however, do not enable us to express in a simple way the corresponding value of $n$ in terms of other quantities. Thus we may take a number of points on the path of a molecule subject to any condition we please, join consecutive points by straight lines, and suppose that the molecule in its migration passes along these straight lines instead of along its actual path. The points, it should be noted, need not even lie on the actual path of the molecule. The straight lines thus obtained constitute, as usual, the representative diffusion paths of a molecule under the stated conditions.

Suppose for example that the points are taken on the path of a molecule, and are so selected that the diffusion paths are grouped about a mean path $l_{\delta r}^{\prime}$ of any chosen length according to Clausius' distribution law given in Section 31, and that any direction of a path in space is equally probable. It follows then from the investigation in Section 42 that the expression for the coefficient of diffusion of a dilute solution of molecules $r$ in $e$ is the same in form as the coefficient given by equation (129), or

$$
D_{r}=\frac{n_{r}^{\prime} l_{\delta r}^{\prime}}{N_{r}}, \quad \text {. . . . . }
$$

where $l_{\delta r}^{\prime}$ has replaced $l_{\delta r}$, and $n^{\prime}{ }_{r}$ the number of representative paths cutting a square $\mathrm{cm}$. in one direction has replaced $n_{r}$. We may give $l_{\delta r}^{\prime}$ any value we please above a limit determined later, and determine the corresponding value of $n^{\prime}{ }_{r}$ from the equation. Since $n^{\prime}{ }_{r}$ is a measure of the chance of a molecule crossing a plane one square $\mathrm{cm}$. in area in moving along its representative path, it follows from the foregoing equation that this chance is inversely proportional to $l^{\prime}{ }_{\delta}$. 
The quantity $n_{r}^{\prime}$ in equation (170) may be expressed in terms of other quantities which are of interest. Thus if $V_{\delta r}^{\prime}$ denote the average velocity a molecule would have if it passed over its representative path, instead of over its actual path, it can be shown that

$$
n_{r}^{\prime}=\frac{N_{r} V_{\delta r}^{\prime}}{3}
$$

along the same lines as equation (35) was obtained. We also have directly that

$$
V_{\delta r}^{\prime}=\frac{l_{\delta r}^{\prime}}{t_{\delta r}^{\prime}}
$$

where $t^{\prime}{ }_{\delta r}$ denotes the average time it would take the molecule to pass over the average diffusion path $l_{\delta r}^{\prime}$, if it passed over its representative paths. It should be noted that $V^{\prime}{ }_{\delta r}$ is not equal to the total average velocity $V_{t r}$ of the molecule unless $l_{\delta r}^{\prime}$ is equal to $l_{\delta r}$. By means of the foregoing two equations equation (170) may be written in the forms

$$
D_{r}=\frac{V_{\delta r}^{\prime} l_{\delta r}^{\prime}}{3}, \ldots . . . .
$$

and

$$
D_{r}=\frac{1}{3} \frac{\left(l^{\prime}{ }_{\delta r}\right)^{2}}{t^{\prime}{ }_{\delta r}} . \quad . \quad . \quad \cdot
$$

In these equations we may give $l_{\delta r}^{\prime}$ any value we please above a limit which will be obtained presently and determine from them the corresponding values of $V^{\prime}{ }_{\delta r}$ and $t^{\prime}{ }_{\delta r}$.

Since the path of a particle is undulatory, and the points locating the diffusion paths lie upon the actual path of the particle, it is evident that the smaller $l^{\prime}{ }_{\delta r}$ is taken the nearer is th" representative path equal to the actual path, and the nearer $V^{\prime}{ }_{\delta r}$ approaches $V_{t r}$ the total average velocity of the molecule. Therefore $V^{\prime}{ }_{\delta r}$ cannot be smaller than $V_{t r}$, and $l_{\delta r}^{\prime}$ therefore according to equation (171) not smaller than 
$3 D_{r} / V_{t r}$. This limiting value of $l_{\delta r}^{\prime}$ corresponds to the value of the diffusion path $l_{\delta r}$ defined in Section 41, since the representative and actual molecular paths are the same in length. But the points locating the path $l_{\delta r}$ cannot, and do not, lie on the actual path. It follows therefore that under the foregoing conditions $l_{\delta r}^{\prime}$ can have values only which are somewhat larger than the value of $l_{\delta r}$. The paths are evidently of interest only under these conditions, namely in that their points of location lie on the actual path of the molecule.

If a molecule in a substance moved along a straight line with a constant velocity, the period $t^{\prime}{ }_{\delta r}$ would be proportional to $l_{\delta r}^{\prime}$, instead of proportional to $\left(l_{\delta r}^{\prime}\right)^{2}$ as indicated by equation (172). It follows therefore that a molecule pursues a zigzag course in a substance. It is interesting to illustrate equation (172) by means of a diagram in this connection. Thus let $a b c d$ in Fig. 18 denote the actual path of a molecule and $a c$ and $a d$ two selected mean diffusion paths of which ad has double the length of $a c$. Now it follows from equation (172) that if the molecule traveled over its representative paths, the time taken in passing over the path $a d$ is $2^{2}$ or 4 times the time taken in passing over the path $a c$. And since the molecule takes the same time in passing over its actual path as over its representative path it follows from the figure that the molecule on the average takes times in the ratio of 1 to 4 in passing over the actual paths $a b c$ and $a b c d$, where $a d=2 a c$.

It is obvious that we may substitute $n_{r}^{\prime}, n_{e}^{\prime}, l_{\delta r}^{\prime}$, and $l_{\delta e}^{\prime}$ for $n_{r}, n_{e}, l_{\delta e}$, and $l_{\delta e}$ in the general diffusion equation (126), where the former symbols have meanings of the nature just considered. The equation may be given forms involving $V_{\delta r}^{\prime}, V_{\delta e}^{\prime}, t_{\delta r}^{\prime}$, and $t_{\delta e}^{\prime}$ similarly as just shown.

The points on the actual path of a molecule which indicate the location of the diffusion paths may be selected in a different way than the foregoing, which is simpler and physically more definite. Thus we may select the points 
so that each line joining two consecutive points is equal to the same length instead of being grouped about a mean path according to Clausius' law, and that any direction of a line in space is equally probable. The deduction of the diffusion equation is then simplified since Clausius' probability factor need not be introduced, and therefore no integration with respect to $z$ is necessary. The integral with respect to $\theta$ (Section 42) is the same as before, and

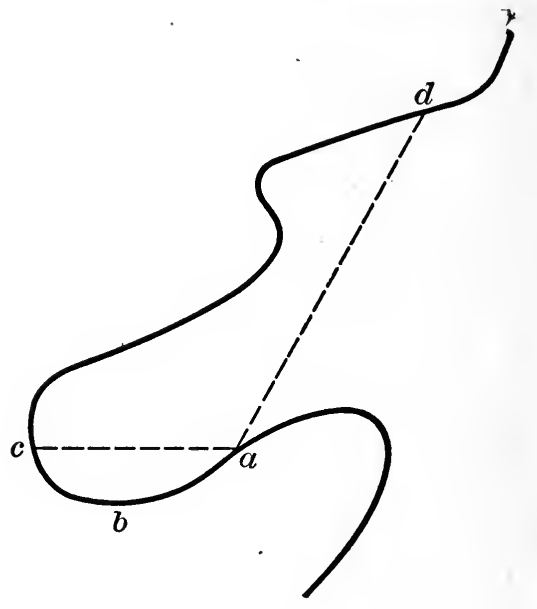

FIG. 18.

introduces the factor $\frac{1}{2}$ into the right-hand side of the diffusion equation, which otherwise would be cancelled by the foregoing integral. The coefficient of diffusion in this case for a dilute solution is therefore given by

$$
D_{r}=\frac{1}{2} \frac{n_{r}^{\prime \prime} l_{\delta r}^{\prime \prime}}{N_{r}}, \quad \text {. . . . . }
$$

where $l^{\prime \prime}{ }_{\delta r}$ denotes the selected diffusion path, and $n^{\prime \prime}{ }_{r}$ the number of diffusion paths passing through an area of 
one square $\mathrm{cm}$. in one direction per second. The foregoing equation may be written in the forms

$$
D_{r}=\frac{V^{\prime \prime}{ }_{\delta r} l^{\prime \prime}{ }_{\delta r}}{6}, \quad . \quad . \quad . .
$$

and

$$
D_{r}=\frac{1}{6} \frac{\left(l^{\prime \prime}{ }_{\delta r}\right)^{2}}{t_{\delta r}^{\prime \prime}}
$$

similarly as before, where $V^{\prime \prime}{ }_{\delta}$ denotes the average velocity the molecule would have if it passed along its diffusion paths, and $t^{\prime \prime}{ }_{\delta r}$ the average time it would take to pass over a single diffusion path. It can be shown similarly as before that $l^{\prime \prime}{ }_{\delta r}$ can have values only somewhat greater than the average diffusion path $l_{\delta r}$.

The general diffusion equation (corresponding to equation (126)) may be written in this case

$$
\delta_{r}=\frac{\vartheta_{e}}{N_{r} \vartheta_{r}+N_{e} \vartheta_{e}}\left\{\frac{\left(l^{\prime \prime}{ }_{\delta r}\right)^{2} N_{e}}{6 t^{\prime \prime}{ }_{\delta r}} \frac{d N_{r}}{d x}+\frac{\left(l^{\prime \prime}{ }_{\delta e}\right)^{2} N_{r}}{6 t^{\prime \prime}{ }_{\delta e}} \frac{d N_{e}}{d x}\right\},
$$

where the meaning of the different symbols is evident from what has gone before.

The points on the path of a molecule locating the diffusion paths may be selected in a third way which is of interest. Thus the period the molecule takes to pass from one point to the next may be taken the same. Let us suppose in this connection that of $N_{r}$ molecules $r$ per cubic $\mathrm{cm}$. $n_{\tau_{1}}$ have a diffusion path $l_{1}$ and $n_{r_{2}}$ a diffusion path $l_{2}$, and so on, corresponding to the period $t^{\prime \prime \prime}{ }_{\delta r}$. We may consider each of these sets of molecules separately and apply the preceding result to obtain expressions for the diffusion. On taking into account that the concentration gradient corresponding to the molecules $n_{r_{1}}$ is $\frac{n_{r_{1}}}{N_{\tau}} \frac{d N_{r}}{d x}$, and that corresponding to the molecules $n_{r_{2}}$ is $\frac{n_{r_{2}}}{N_{r}} \frac{d N_{r}}{d x}$, and so on, it 
follows at once that the coefficient of diffusion of a dilute solution is given by

$$
D_{r}=\frac{1}{6 t^{\prime \prime \prime}{ }_{\delta r}}\left\{\frac{n_{r_{1}} l_{1}^{2}+n_{r_{2}} l_{2}^{2}+\ldots}{N_{r}}\right\}=\frac{1}{6} \frac{\left(\overline{l^{\prime \prime \prime}{ }_{\delta r}}\right)^{2}}{t^{\prime \prime \prime}{ }_{\delta r}}, .
$$

where $\left(\overline{l^{\prime \prime \prime}{ }_{\delta r}}\right)^{2}$ denotes the mean of the squares of the diffusion paths. The corresponding general form of the diffusion equation may now be written down without any difficulty.

It will readily be recognized now that the points locating the diffusion paths may be selected in other ways, in fact according to any law we please. The foregoing three ways are, however, the only ones of special interest and importance.

An interesting and important feature of the foregoing equations is the fact that either the period or the diffusion path may be given any value we please. We may therefore immediately calculate the diffusion path corresponding to a given period, or vice versa. These values furnish interesting information about molecular motion. A set of such calculations has been carried out in the next Section in connection with similar equations involving viscosity.

The dependence of the diffusion path, or the period, in equation (175) on more fundamental quantities may be obtained by equating the coefficient of diffusion given by the equation and that given by equation (129), and substituting for $l_{\delta r}$ from equations (130), which gives

$$
t^{\prime \prime}{ }_{\delta r}=\frac{N_{r} N_{e r}\left(v_{r}-b_{r}^{\prime}\right)\left(l^{\prime \prime}{ }_{\delta r}\right)^{2}}{6 n_{r} v_{r} \kappa_{\delta r}^{\prime} N\left(1+\Phi_{\delta r}^{\prime}\right)} \text {. . . . }
$$

This equation may also be written

$$
t^{\prime \prime}{ }_{\delta r}=\frac{N_{e r}\left(v_{r}-b_{r}^{\prime}\right)\left(l^{\prime \prime}{ }_{\delta r}\right)^{2}}{2 V_{{ }_{r} v_{r} \kappa^{\prime}{ }_{\delta r} N\left(1+\Phi^{\prime}\right)_{\delta r}}}
$$

by means of equation (35). Thus for a selected constant value of $l^{\prime \prime}{ }_{\delta r}$ the period varies inversely as the total average velocity $V_{t r}$ of a molecule $r$, as we might expect. The 
existence of the apparent volume $b^{\prime}{ }_{r}$ of the molecules $r$ and $e$ has the effect of decreasing the period from what it otherwise would be. This could not be recognized directly. The existence of molecular interference has the same effect since $\Phi_{\delta r}^{\prime}$ is positive. Also an increase in $\kappa_{\delta r}^{\prime}$, which is proportional to the diffusion path in the gaseous state at standard pressure, tends to decrease the period.

The period may be expressed in terms of the partial intrinsic pressures and other quantities on eliminating $n_{r}$ from equation (178) by means of equation (152).

The variation of $l^{\prime \prime}{ }_{\delta r}$ for a given selected constant period $t^{\prime \prime}{ }_{\delta r}$ is obtained by solving the foregoing equations with respect to $l^{\prime \prime}{ }_{\delta r}$.

Equations similar to the foregoing may be obtained corresponding to a mixture which is not dilute. Thus on equating the expressions for $\delta_{r}$ given by equations (126) and (176), eliminating $l_{\delta r}$ and $l_{\delta e}$ by means of equations (130), and equating the factors of $\frac{d N_{r}}{d x}$ and $\frac{d N_{e}}{d x}$ separately to zero, which holds since the equation is an identity, we obtain in the case of the molecules $r$ an equation which is the same as equation (178) having the factor

$$
\frac{N_{r} \vartheta_{r}+N_{e} \vartheta_{e}}{N_{e} \vartheta_{e}}
$$

introduced into the right-hand side. Thus we see that in the case of a solution of molecules $r$ in $e$ which is not dilute, an increase in the number of molecules $r$ relative to the molecules $e$ increases the period $t^{\prime \prime}{ }_{\delta r}$. The existence of the external molecular volume $\vartheta_{e}$ of the molecules $e$ decreases the period from what it otherwise would be, while the external molecular volume $\vartheta_{r}$ of the molecules $r$ increases it.

The periods corresponding to the selected paths, or vice versa, may approximately be calculated from equations (178) and (179) on determining the approximate values 
of the quantities on their right-hand sides by means of the method described in Section 42.

\section{Extended Forms of the Viscosity Equations.}

The equations for the viscosity given in Sections 34 and 35 may be given extended forms similarly as the diffusion equations in the previous Section. Points may be selected on the actual path of each molecule and these joined, and the supposition made that momentum is given to the substance parallel to its motion, or abstracted from the substance, at these points only by the molecule. The line joining two consecutive points may be called a momentum transfer distance under the stated conditions. The points of location of the transfer distances may be selected according to any given law; there are three ways only, however, which are of interest, and which were used in connection with diffusion. Thus we may suppose that the transfer distances are grouped about their mean according to Clausius' law; or are equal to each other; or correspond to the same period for the molecule to pass from one point to the consecutive point, the points in each case being so selected that any direction of a line of given length is equally probable.

In the first case the form of the viscosity equation remains the same as that of equation (83), and for a pure substance $r$ may be written

$$
\eta=n_{r}^{\prime} m_{a r} l_{\eta r}^{\prime}
$$

where $l_{\eta r}^{\prime}$ denotes the mean momentum transfer distance in this case, and $n_{r}^{\prime}$ the number of representative paths crossing a square $\mathrm{cm}$. from one side to the other per second. The latter quantity is given by

$$
n_{r}^{\prime}=\frac{N_{r} V_{\eta_{r}}}{3}
$$


where $V_{\eta r}$ denotes the velocity the molecule would have if it moved along its representative path, this quantity being directly given by

$$
V_{\eta r}=\frac{l_{\eta r}^{\prime}}{t_{\eta r}^{\prime}}
$$

where $t^{\prime}{ }_{\eta r}$ denotes the period of the average transfer distance. Equation (180) may therefore be written in the more important form

$$
\eta=\frac{N_{r} m_{a r}}{3} \frac{\left(l_{\eta_{r}}^{\prime}\right)^{2}}{t^{\prime}{ }_{\eta r}} . . . . .
$$

The equation for the viscosity of a mixture of molecules $r$ and $e$ is accordingly given by

$$
\eta=\frac{N_{r} m_{a r}}{3} \frac{\left(l_{\left.\eta^{\prime}\right)^{2}}^{2}\right.}{t^{\prime}{ }_{\eta r}}+\frac{N_{e} m_{a e}}{3} \frac{\left(l_{\eta e}^{\prime}\right)^{2}}{t^{\prime}{ }_{\eta e}} . . .
$$

If the transfer distances are taken equal to each other, it can be shown similarly as in the previous Section that the corresponding viscosity equations are the same in form as equations (181) and (182) having the factor $\frac{1}{2}$ introduced into each right-hand side, that is, we may write

and

$$
\eta=\frac{N_{r} m_{a r}}{6} \frac{\left(l^{\prime \prime}{ }_{\eta r}\right)^{2}}{\overline{i^{\prime \prime}{ }_{\eta r}}}, \ldots . . .
$$

$$
\eta=\frac{N_{r} m_{a r}}{.6} \frac{\left(l^{\prime \prime}{ }_{\eta r}\right)^{2}}{\overline{t^{\prime \prime}{ }_{\eta r}}}+\frac{N_{e} m_{a e}}{6} \frac{\left(l^{\prime \prime}{ }_{\eta e}\right)^{2}}{\overline{t^{\prime \prime}{ }_{\eta e}}}
$$

where $l^{\prime \prime}{ }_{\eta r}$ denotes the selected constant momentum transfer distanoe of a molecule $r, \overline{t^{\prime \prime}}{ }_{\eta r}$ the mean of its periods, while the other symbols referring to the molecules $e$ have similar meanings.

If the average period for a pure substance in equation (183) is taken equal to the period in the case of equation (175) applied to the diffusion of a molecule in molecules of 
the same kind, and this period eliminated from the two equations, we obtain the equation

$$
\frac{l^{\prime \prime}{ }_{\delta r}}{l^{\prime \prime}{ }_{\eta \tau}}=\sqrt{\frac{N_{r} m_{a r} D_{r}}{\eta}} . \cdots .
$$

If the nature of the motion of a molecule were not altered by a shearing motion given to the substance, $l^{\prime \prime}{ }_{\eta r}$ would be equal to $l^{\prime \prime}{ }_{\delta r}$, which corresponds to the normal motion of a molecule. The foregoing equation, however, indicates that this does not hold, as we might expect, and expresses the effect of shearing motion on the value of $l^{\prime \prime}{ }_{\eta r}$ for unit velocity gradient in the substance.

Since the viscosity of a gas is independent of its pressure, or the molecular concentration, it follows from equation (183) that the value of the average period $\overline{t^{\prime \prime}{ }_{\eta r}}$ for a constant value of $l^{\prime \prime}{ }_{\eta r}$ is proportional to the molecular concentration $N_{r}$. This shows that the molecules in their encounters deflect each other from their paths, and therefore the actual path of a molecule between two points is greater than the straight line joining them. We may therefore say that the period a molecule takes to traverse its curved and zigzag path between two points a constant distance apart in a gas whose pressure is varied, is proportional to its chance of encountering another molecule.

The viscosity of a liquid is greater than that corresponding to the gaseous state, and hence according to equation (183) the average period $\overline{t^{\prime \prime}{ }_{\eta r}}$ for a constant value of $l^{\prime \prime}{ }_{\eta r}$ in the case of a liquid, is smaller than proportional to $N_{r}$, or smaller than the chance of the molecule encountering another molecule along its path. This would be the case if the average velocity of a molecule in the liquid state is greater than in the gaseous, which would decrease the time it takes the molecule to pass over its path. This fits in with results obtained previously. 
It is of interest to obtain by means of equation (183) for different substances the average periods ${\overline{t^{\prime \prime}}}_{\eta r}$ that a molecule takes to pass from one point to another one mm. apart, or corresponding to $l^{\prime \prime}{ }_{\eta r}=.1 \mathrm{~cm}$. Table XXVIII gives the values of the periods in the case of $\mathrm{CO}_{2}$ at different pres-

\section{TABLE XXVIII}

\begin{tabular}{|c|c|c|c|c|}
\hline \multicolumn{5}{|c|}{ VALUES of $\overline{t^{\prime \prime}}$ CoRresponding to $l^{\prime \prime}{ }_{\eta r}=.1 \mathrm{~cm}$. } \\
\hline \multicolumn{5}{|c|}{$\mathrm{Co}_{2}$ aT $40^{\circ} \mathrm{C}$} \\
\hline $\begin{array}{c}p \text { in } \\
\text { atmos. }\end{array}$ & $\begin{array}{l}\text { Volume } \\
\text { of a gram } \\
\text { molecule. }\end{array}$ & $\eta 10^{8}$. & $N_{r} 10^{21}$ & $\begin{array}{r}\overline{t^{\prime \prime} \eta r} \text { in } \\
\text { seconds. }\end{array}$ \\
\hline 70 & 245.7 & 200 & 2.52 & 1.49 \\
\hline 80 & 172.2 & 218 & 3.61 & 1.95 \\
\hline 85 & 130.7 & 269 & 4.74 & 2.08 \\
\hline 94 & 85.35 & 414 & 7.26 & 2.08 \\
\hline 100 & 78.96 & 483 & 7.86 & 1.92 \\
\hline 112 & 73.24 & 571 & 8.48 & 1.75 \\
\hline \multicolumn{5}{|c|}{ Ether } \\
\hline$t^{\circ} \mathrm{C}$ & p. & $\eta 10^{6}$. & $N_{r} 10^{21}$ & $\begin{array}{c}{\overline{t^{\prime \prime}}}_{\eta r} \text { in } \\
\text { seconds. }\end{array}$ \\
\hline 13.5 & .7214 & 1779 & 6.05 & .67 \\
\hline 99 & .6421 & 1133 & 5.39 & .94 \\
\hline \multicolumn{5}{|c|}{ ChLOROFORM } \\
\hline 0 & 1.5264 & 3827 & 7.93 & .66 \\
\hline 60 & 1.4108 & 2791 & 7.33 & .84 \\
\hline \multicolumn{5}{|c|}{ Benzene } \\
\hline 15.4 & .8840 & 4387 & 7.38 & .36 \\
\hline 78.8 & .8145 & 3000 & 6.65 & .45 \\
\hline
\end{tabular}




\section{MISCELLANEOUS APPLICATIONS, CONNECTIONS}

sures at the temperature $40^{\circ} \mathrm{C}$, and of three liquids at different temperatures. The period of a molecule of $\mathrm{CO}_{2}$ when it does not obey the gas laws evidently does not depend much on the molecular concentration, the effect of the increase of concentration being probably more or less balanced by the increase in molecular velocity. The order of the period is two seconds. In the case of liquids the period is increased with an increase of temperature, as we would expect, since the concentration is thereby decreased which decreases the molecular velocity. Its value appears to decrease with an increase in the molecular weight of the liquid, and is of the order of one second.

In the case of a gas $r$ at standard temperature and pressure the period is under the foregoing conditions given by the equation

$$
\overline{t^{\prime \prime}{ }_{\eta r}}=\frac{4.5 \times 10^{-7} m_{r}}{\eta}, \quad \ldots . .
$$

where $m_{r}$ denotes the relative molecular weight, and $\eta$ the viscosity which in this case is independent of the pressure. An idea of the dependence of the period on the nature of the gas may be obtained from an inspection of Table $\mathrm{X}$ which contains values of $\eta$ and $m$ for a number of different gases.

If the period is kept constant it can be shown similarly as in the previous Section that the visoosity in the case of a pure substance $r$ is given by

$$
\eta=\frac{N_{r} m_{a r}}{6} \frac{\left(\overline{l^{\prime \prime \prime}{ }^{\prime}}\right)^{2}}{t^{\prime \prime \prime}{ }_{\eta^{r}}}, \ldots . . .
$$

where $\left(\overline{l^{\prime \prime \prime}{ }_{\eta \tau}}\right)^{2}$ denotes the mean of the squares of the transfer distances corresponding to the constant period $t^{\prime \prime \prime}{ }_{\eta r}$, and an equation consisting of similar terms may be obtained for a mixture of substances. 
It can be shown in the same way as in the previous Section that the smallest admissible values of $l^{\prime}{ }_{\eta r}, l^{\prime \prime}{ }_{\eta r}$, and $\overline{l^{\prime \prime \prime} \eta_{r}}$ are somewhat larger than the value of $l_{\eta r}$ defined in Section 33.

The dependence of the extended transfer distances and periods on the nature of molecular interaction is obtained on equating the expression for the viscosity obtained in this Section with those obtained in Section 34. Thus in the case that the transfer distance is kept a constant length we obtain from equation (183) and equations (90), (91), and (92), the equations

$$
\begin{aligned}
& \overline{t^{\prime \prime}{ }_{\eta r}}=\frac{1}{6} \frac{v_{r}-b_{r}}{v_{r}} \frac{N_{r}\left(l^{\prime \prime}{ }_{\eta r}\right)^{2}}{n_{r} v_{r} \kappa_{\eta r}\left(1+\Phi_{\eta r}\right)}, . . \\
& \overline{t^{\prime \prime}{ }_{\eta r}}=\frac{1}{2} \frac{v_{r}-b_{r}}{v_{r}} \frac{\left(l^{\prime \prime}{ }_{\eta r}\right)^{2}}{V_{t r} v_{r} \kappa_{\eta r}\left(1+\Phi_{\eta r}\right)}, . . \\
& \overline{t^{\prime \prime}{ }_{\eta r}}=\frac{1}{12} \frac{N_{r} A_{r}\left(l^{\prime \prime}{ }_{\eta r}\right)^{2}}{\left(P_{n r}+p_{r}\right) v_{r} \kappa_{\eta r}\left(1+\Phi_{\eta r}\right)}, . .
\end{aligned}
$$

on adding the suffix $r$ to the symbels of the latter equations in order to bring the notation into line with the former equation, and taking into account that $v_{r} N_{r} m_{a r}=m_{r}$, where

$$
A_{r}=5.087 \times 10^{-20} \sqrt{T m_{r}} .
$$

It will be seen from these equations that the apparent molecular volume $b_{r}$, and the interference function $\Phi_{\eta r}$ (which is positive), decreases the period from what it otherwise would be. Thus the existence of molecular forces of repulsion, which prevents the molecules approaching each other within any degree of closeness, and the existence of interference of the molecules of the substance with two interacting molecules, have the effect of decreasing the period. An increase in the total average velocity $V_{t r}$ has the effect of decreasing the period, as is also directly evident. 
In the case of a substance not obeying the gas laws an increase in velocity at constant temperature, it may be noted, may be brought about by an increase in the density of the substance (Sections 17 and 29). An increase in $\kappa_{\eta r}$, which decreases with an increase of the molecular mass, has the effect of decreasing the period, and this also holds for the volume $v_{r}$ of a gram molecule. An increase in the molecular forces of attraction would give rise to an increase in the intrinsic pressure $P_{n r}$, and in the number $n_{r}$ of molecules crossing a square $\mathrm{cm}$. per second, and thus give rise to a decrease of the period. Equation (189) of the three equations (188), (189), and (190), indicates best the dependence of the period on the temperature at constant volume. It decreases with an increase of temperature, since $V_{t r}, \kappa_{\eta r}$, and $\Phi_{\eta r}$ increase with an increase of temperature at constant volume (Sections 16,17 , and 34 ), while $b_{r}$ is probably approximately independent of the temperature.

The dependence of $l^{\prime \prime}{ }_{\eta r}$ on the fundamental properties of a substance for a constant period is obtained on solving the foregoing equations with respect to $l^{\prime \prime}{ }_{\eta r}$. It is evident that the effect of the changes in the quantities considered on the quantity $l^{\prime \prime}{ }_{\eta r}$ is opposite in direction, and less (through the extraction of square roots on solving the equations as indicated) than in the case of the period.

In order to obtain the dependence of the periods on other quantities in the case of a mixture of molecules $r$ and $e$, each of the terms on the right-hand side of equation (184) is equated with one of the two terms to which it corresponds on the right-hand side of equation (98), and the resulting equation transformed by means of equations (99) and (100). Equations similar in form to equations (188), (189), and (190) will be obtained in this way.

Approximate values of the periods corresponding to given transfer distances, or vice versa, in the case of a mixture, may be obtained from these equations on obtain- 
ing approximate values of the other quantities they contain in the way described in Section 35 .

In connection with the foregoing investigation the following remarks may help to clear up any difficulties encountered. If we take a plane parallel to the motion of the substance corresponding to the velocity $V_{1}$, it follows from considerations of equilibrium that the algebraical momentum per molecule parallel to the plane on the average is equal to $V_{1} m_{a}$. Therefore on considering two planes corresponding to the velocities $V_{1}$ and $V_{2}$ of the substance, it follows that the molecules crossing each plane in the same direction will each have on the average its momentum parallel to the planes changed from $V_{1} m_{a}$ to $V_{2} m_{a}$ on passing from one plane to the other. This will, of course, not hold for each molecule considered independently. These considerations show that we may suppose that momentum is given by a molecule to the medium and abstracted from it in these planes only, which may be taken any distance apart. This may be illustrated by considering a number of similar parallel planes corresponding to the velocities $V_{1}, V_{2}, \ldots V_{e}$ of the substance. The momentum transferred from the first to the last plane by a molecule is equal to $\left\{\left(V_{1}-V_{2}\right)+\left(V_{2}-V_{3}\right)\right.$ $\left.+\ldots\left(V_{e-1}-V_{e}\right)\right\} m_{a}$, or equal to $\left(V_{1}-V_{e}\right) m_{a}$, and thus the intermediate planes have no effect on the amount of momentum transferred from the first of the planes to the other. It follows, therefore, that a molecule crossing one of the foregoing planes may, or may not, be taken to change its viscosity momentum in crossing it, just as we please. Also a molecule may recross a number of times each of two consecutive planes between the two points lying on the planes at which only it is supposed to change its viscosity momentum. The foregoing planes may therefore be taken to indicate the location of the various points defining the molecular paths used in the previous investigation; and hence it follows that these paths are permissible to use. 
It should be pointed out, however, that the representative molecular path probably cannot assume all numerically possible values between two given limits, or that it is probably a recurring discontinuous function. It can easily be shown, for example, that the values of the path $l$ for a part which is a straight line do not fit in with the relation $l^{2} / t=$ constant. But the total region of discontinuity, if not zero, is probably small in comparison with the remaining region.

\section{Extended Forms of the Heat Conduction Equations.}

Extended heat conduction equations may be obtained along the same lines as the extended viscosity and diffusion equations in the preceding two Sections. We may suppose as before that the heat transfer distances are defined by points distributed according to any given law on the paths of the molecules. The transfer distances are of interest only, however, when the points are distributed according to one of the three ways already used.

If the transfer distances are grouped about a mean according to Clausius' law the heat conductivity equation for a pure substance $r$ may immediately be written

$$
C=n^{\prime}{ }_{r} m_{a r} S_{g r} l_{c r}^{\prime}, \quad . \quad . \quad . \quad .
$$

according to Section 38, where $l_{c r}^{\prime}$ denotes the average heat transfer distance which may have any value above a certain limit to be determined presently, $n^{\prime}$ r denotes the number of transfer distances crossing a square $\mathrm{cm}$. in one direction per second, $S_{g r}$ denotes the specific heat at constant pressure per gram and $m_{a r}$ the absolute molecular weight. If $V_{c r}$ denotes the average velocity a molecule would have if it passed along its successive transfer distances, and $t_{e r}$ the time it would take to pass over the average transfer 
distance (which is the same as the time taken to pass over the actual path), we have

$$
V_{c r}=\frac{l_{c r}^{\prime}}{t_{c r}}, \text { and } n_{r}^{\prime}=\frac{N_{r} V_{c r}}{3}
$$

Hence equation (191) may be written in the form

$$
C=\frac{N_{r} m_{a r} S_{g r}}{3} \frac{\left(l_{c r}^{\prime}\right)^{2}}{t_{c r}^{\prime}}, \quad \ldots .
$$

which is more important. The heat conduction equation for a mixture of molecules $r$ and $e$ may now immediately be written down.

If the distance between each pair of consecutive points is taken the same and equal to $l^{\prime \prime}{ }_{c r}$, and $\overline{t^{\prime \prime}{ }_{c r}}$ is the average period of the transfer distance, it can be shown along the same lines of reasoning as contained in the preceding two Sections that

$$
C=\frac{N_{r} m_{a r} S_{g r}}{6} \frac{\left(l^{\prime \prime}{ }_{(r)}\right)^{2}}{\overline{t^{\prime \prime}{ }_{r}}}, \ldots \cdot \cdot \cdot \cdot \cdot \cdot
$$

for a pure substance, and

$$
C=\frac{N_{r} m_{a r} S_{g r}}{6} \frac{\left(l_{c r}^{\prime \prime}\right)^{2}}{\overline{t^{\prime \prime}}{ }_{r}}+\frac{N_{e} m_{a e} S_{0 e}}{6} \frac{\left(l^{\prime \prime}{ }_{c e}\right)^{2}}{\overline{t^{\prime \prime}{ }_{e}}}, .
$$

for a mixture of molecules $r$ and $e$.

On the other hand if the period $t^{\prime \prime}{ }_{r}{ }_{r}$ is kept the same it can be shown along similar lines as before that

$$
C=\frac{N_{r} m_{a r} S_{\theta r}}{6} \frac{\left(\overline{l^{\prime \prime \prime}{ }_{r}}\right)^{2}}{t^{\prime \prime \prime}{ }_{r}}, \ldots . . .
$$

in the case of a pure substance $r$, where $\left(\overline{l^{\prime \prime \prime}{ }_{C T}}\right)^{2}$ denotes the mean of the squares of the corresponding heat transfer dis- 
tances. An equation consisting of the sum of similar terms holds for the coefficient of conductivity in the case of a mixture.

It can be shown along the same lines as in the preceding two Sections that the least admissible values of $l^{\prime}{ }_{c r}, l^{\prime \prime}{ }_{c r}$, and $\overline{l^{\prime \prime \prime}{ }_{c r}}$ are somewhat larger than the value of $l_{c}$ as defined in Section 38.

If the period in equation (193) is taken equal to the period in equation (183) and it is eliminated from the equations, the equation

$$
\frac{l^{\prime \prime}{ }_{\eta r}}{l^{\prime \prime}{ }_{c r}}=\sqrt{\frac{\eta S_{o r}}{C}}
$$

is obtained. This equation gives some information about the effect of a shearing motion of a substance, and of a flow of heat, on the nature of the motion of a molecule. For the quantity $l^{\prime \prime}{ }_{\eta r}$ refers to the motion of a molecule in a substance undergoing a shearing motion corresponding to a unit velocity gradient, and the quantity $l^{\prime \prime}{ }_{c r}$ to the motion of a molecule in a substance through which a flow of heat takes place corresponding to a unit heat gradient. If the molecular motion were not affected by these conditions $l^{\prime \prime}{ }_{c r}$ would be equal to $l^{\prime \prime}{ }_{n e}$. But this is evidently not the case according to the foregoing equation, since the term under the radical is not equal to unity. According to Section 38 the right-hand side of the equation is larger than unity when the substance is in the liquid state, and smaller than unity when in the gaseous state. Therefore on the average the line joining two points on the path of a molecule in a liquid for a constant period is increased when a velocity gradient exists in the substance in comparison with the magnitude of the line when a heat gradient exists, and the opposite holds in the case of a gas.

The fact that the internal molecular energy of a complex molecule is several times greater than the free kinetic energy 
of translation (Section 13), gives rise to a transference of energy to the medium by the molecule in moving along a heat gradient, or vice versa, which is considerably greater than the change in kinetic energy. This will have the effect of changing the nature of the path of the molecule considerably from what it would be if the molecule possessed no energy apart from its kinetic energy of translation. The distortion of the molecular path by a heat gradient is probably in the main caused in this way.

If the periods are taken equal in equations (193) and (175) and eliminated we obtain the equation

$$
\frac{l^{\prime \prime}{ }_{\delta r}}{l^{\prime \prime}{ }_{c r}^{\prime}}=\sqrt{\frac{N_{r} m_{a r} S_{0 r} D_{r}}{C}}
$$

The quantity $l^{\prime \prime}{ }_{\delta r}$ may be taken to correspond to the diffusion of a molecule in a substance of the same kind instead of in a mixture in which molecules $r$ possess a concentration gradient. The motion of a molecule in the former case is normal. It would be interesting to test the equation under these conditions.

The heat transfer distance and its period may be connected with more fundamental quantities in the same way as similar quantities in the preceding two Sections in the case of viscosity and diffusion.

It will be seen that the diffusion, viscosity, and heat conduction expressions given in this and the preceding two Sections are each of the form $l^{2} / t$ multiplied by a factor which depends on the conditions under which the molecules are undergoing motion of translation. Each of the quantities $l$ and $t$ may be taken to a certain extent as arbitrary, but not at the same time, since one determines the other.

A set of equations for the diffusion, viscosity, and heat conduction of substances will now be developed, which are similar to the equations obtained in this and the preceding 
two Sections, and which involve the projection of the moticn of a molecule along a line.

\section{The Constant Period Displacement Dif-} fusion Equation and its Applications.

Let us take an imaginary plane for reference at right angles to the diffusion in a heterogeneous mixture and determine the number of molecules of one kind that drift across it per square $\mathrm{cm}$. per second. The motion of the molecules will be expressed in terms of their displacement at right angles to the foregoing plane for a selected period $t$. It will be evident that in each element of volume half of the molecules during the time $t$ undergo a displacement in one direction, while the other half undergoes a displacement in the opposite direction. Suppose that $n_{r_{1}}, n_{r_{2}}, n_{r_{3}}$, ..., molecules $r$ whose displacements are respectively $d_{r_{1}}, d_{r_{2}}, d_{r_{3}}, \ldots$, during the period $t_{r}$ cross the plane per square $\mathrm{cm}$. in the direction of their diffusion-say from top to bottom. The molecules of displacement $d_{r_{1}}$ come from a cylinder of height $d_{r_{1}}$ and unit cross-section standing on the plane, while the molecules of displacement $d_{r_{2}}$ come from a cylinder of height $d_{r_{2}}$ standing on the plane, etc. Therefore if $n_{r_{1}}^{\prime}, n_{r_{2}}^{\prime}, n_{r_{3}}^{\prime} \ldots$, molecules $r$ of displacements $d_{r_{1}}, d_{r_{2}}, d_{r_{3}}, \ldots$, respectively come from each cubic $\mathrm{cm}$. of the mixture we have

We also have

$$
n_{r_{1}}=n_{r_{1}}^{\prime} d_{r_{1}}, n_{r_{2}}=n_{r_{2}}^{\prime} d_{r_{2}} \text {, etc. }
$$

$$
2\left(n_{r_{1}}^{\prime}+n_{r_{2}}^{\prime}+\ldots\right)=N_{r}, \quad \text {. . . }
$$

since each molecule undergoes a displacement during the period $t_{r}$, one-half of the molecules undergoing a displacement in one direction and the other half undergoing a displacement in the other direction, where $N_{r}$ denotes the con- 
centration of the molecules $r$. The total number of molecules $r$ which cross the plane in the direction of diffusion, or from top to bottom, during the time $t_{r}$ is therefore equal to

$$
n_{r_{1}}+n_{r_{2}}+n_{r_{3}}+\ldots=n_{r_{1}}^{\prime} d_{r_{1}}+n_{r_{2}}^{\prime} d_{r_{2}}+n_{r_{3}} d_{r_{3}}+\ldots
$$

But molecules $r$ also cross the plane in the opposite direction. Similarly as before the molecules of displacement $d_{r_{1}}$ come from a cylinder of height $d_{r_{1}}$ and unit cross-section standing on the plane, and the molecules of displacement $d_{r_{2}}$ come from a cylinder of height $d_{r_{2}}$ standing on the plane, etc. We may suppose, to simplify matters, that the molecules $r$ of displacement $d_{r_{1}}$ which cross the plane of reference in the direction of the diffusion, or from top to bottom, come from a plane at a distance $d_{r_{1}} / 2$ from the plane of reference, while the molecules of displacement $d_{r_{1}}$ crossing in the opposite direction come from a plane at the distance $d_{r_{1}} / 2$ on the opposite side of the plane of reference. The numbers of these two sets of molecules are proportional to the concentrations of the molecules at the starting planes. If the concentration at the upper starting plane is $N_{r}$, that at the lower plane is $N_{r}-d_{r_{1}} \frac{d N_{r}}{d x}$, where $\frac{d N_{r}}{d x}$ is the concentration gradient of the molecules $r$ measured in the direction of increase of concentration. We may therefore write $K N_{r}$ and $K\left(N_{r}-d_{r_{1}} \frac{d N_{r}}{d x}\right)$ for the numbers of these two sets for migrating molecules, where $K$ denotes an appropriate factor. Since $n_{r_{1}}=K N_{r}$ these numbers may also be written

$$
n_{r_{1}} \text { and } n_{r_{1}}-\frac{n_{r_{1}} d_{r_{1}}}{N_{r}} \frac{d N_{r}}{d x}
$$

or

$$
n_{r_{1}}^{\prime} d_{r_{1}} \text { and } n_{r_{1}}^{\prime} d_{r_{1}}-\frac{n_{r_{1}}^{\prime} d_{r_{1}}^{2}}{N_{r}} \frac{d N_{r}}{d x}
$$




\section{MisCELLANEOUS APPLICATIONS, CONNECTIONS}

since $n_{r_{1}}=n_{r_{1}}{ }_{1} d_{r_{1}}$. Therefore the excess of molecules $r$ of displacement $d_{r_{1}}$ crossing the plane of reference in the direction of diffusion over the molecules $r$ of displacement $d_{r_{1}}$ crossing in the opposite direction in the time $t_{r}$, is equal to

$$
\frac{n_{r_{r}}^{\prime} d_{r_{1}}^{2}}{N_{r}} \frac{d N_{r}}{d x}
$$

Similarly it can be shown that the excess of molecules $r$ of displacement $d_{r_{2}}$ crossing the plane is equal to

$$
\frac{n_{r_{2}}^{\prime} d_{r_{2}}^{2}}{N_{r}} \frac{d N_{r}}{d x}
$$

and so on. Therefore the total excess of molecules $r$ that cross the plane in one second due to the displacements they undergo, is equal to

$$
\frac{\left(2 n_{r_{1}}^{\prime} d_{r_{1}}^{2}+2 n_{r_{2}}^{\prime} d_{r_{2}}^{2}+\ldots\right)}{2\left(2 n_{r_{1}}^{\prime}+2 n_{r_{2}}^{\prime}+\ldots\right)} \frac{1}{t_{r}} \frac{d N_{r}}{d x}=\frac{\overline{d_{r}^{2}}}{2 t_{r}} \frac{d N_{r}}{d x},
$$

by the help of equation (198), where

$$
\frac{\left(2 n_{r_{1}}^{\prime} d_{r_{1}}^{2}+2 n_{r_{2}}^{\prime} d_{r_{2}}^{2}+\ldots\right)}{\left(2 n_{r_{1}}^{\prime}+2 n_{r_{2}}^{\prime}+\ldots\right)}=\overline{d_{r}^{2}}
$$

the mean of the squares of the displacements. Similarly it can be shown that the excess of molecules $e$ that cross the plane in the opposite direction per second due to the displacements they undergo is equal to

$$
\overline{d_{e}^{2}} \frac{d N_{e}}{2 t_{e}},
$$

where $\overline{d^{2}}{ }_{e}$ denotes the mean of the squares of the displacements corresponding to the period $t_{e}$, and $\frac{d N_{e}}{d x}$ the concentration gradient of the molecules $e$ measured in the 
direction of increase of concentration, and thus in the opposite direction that the gradient of the molecules $r$ is measured. Thus on the whole there is a gain of molecules $r$ and $e$ per second below the plane equal to

$$
\frac{\overline{d_{r}^{2}}}{2 t_{r}} \frac{d N_{r}}{d x}-\frac{\overline{d^{2}}}{2 t_{e}} \frac{d N_{e}}{d x}
$$

But the space occupied by these molecules is not zero. A portion of the mixture must therefore be transported bodily across the plane to make room for the foregoing molecules. This may be taken into account in exactly the same way as in Section 42. It will appear from that investigation that the total migration $\delta_{r}$ of molecules $r$ across the plane per second is accordingly given by

$$
\delta_{r}=\frac{\vartheta_{e}}{N_{r} \vartheta_{r}+N_{e} \vartheta_{e}}\left\{\frac{\overline{d_{r}^{2}} N_{e}}{2 t_{r}} \frac{d N_{r}}{d x}+\frac{\overline{d^{2}} N_{r}}{2 t_{e}} \frac{d N_{e}}{d x}\right\},
$$

where $\vartheta_{r}$ and $\vartheta_{e}$ denote the external molecular volumes of gram molecules of molecules $r$ and $e$ in the mixture. The diffusion of the molecules $e$ is obtained by interchanging the suffixes $r$ and $e$ in the foregoing equation.

In the case of a dilute solution of molecules $r$ in $e$ the foregoing equation becomes

$$
\delta_{r}=\frac{\overline{d_{r}^{2}}}{2 t_{r}} \frac{d N_{r}}{d x} \cdot \ldots . \quad . \quad .
$$

The coefficient of diffusion $D_{r}$ in this case is therefore given by

$$
D_{r}=\frac{\overline{d_{r}^{2}}}{2 t_{r}} . \quad \cdot \quad \cdot \quad \cdot \quad \cdot \quad . \quad \cdot
$$

Thus if the molecular displacements in a mixture for a given period could be measured the diffusion could be calculated. 
This is of course impossible in the case of molecules, but can be carried out in the case of Brownian particles. Examples of such investigations will be considered further on.

The foregoing equations are limited according to the following considerations: Suppose that the displacements of the molecules of a cubic $\mathrm{cm}$. of matter were observed for several consecutive periods each equal to $t_{r}$. The value of $\overline{d^{2}}{ }_{r}$ for each set of observations should be the same, since the equations are independent of the time of beginning of a set of observations. Now this is the less likely to be realized the smaller the value of $t_{r}$. Hence there is a limiting value of $t_{r}$ for lower values of which the equations do not hold. This limiting value can definitely be determined as will now be shown. If the path of each molecule consisted of a number of straight lines joined at various angles, and their lengths were grouped about a mean length according to Clausius' probability law given in Section 31, while each inclination of a line in space were equally probable, each line or molecular free path would be independent of the preceding path. The corresponding displacements, or projections of the free paths on to an axis, would therefore also be independent of each other. But this would evidently not hold for displacements corresponding to a fraction of a free path. Now the average of the displacements $d_{r}$, which is given by

$$
\overline{d_{r}}=\left(\frac{2 n_{r_{1}}^{\prime} d_{r_{1}}+2 n_{r_{2}}^{\prime} d_{r_{2}}+\ldots .}{2 n_{r_{1}}^{\prime}+2 n_{r_{2}}+\ldots}\right),
$$

and the average free path $l_{\delta r}$ are connected by the equation

$$
4 \overline{d_{r}}=l_{\delta r} \cdot . \quad . \quad . \quad . \quad .
$$

according to Section 44. Hence it follows that a period $t_{r}$ is inadmissible if the corresponding average displacement has a smaller value than given by the foregoing equation. 
This would be indicated in practice by the values of $t_{r}$ and $\overline{d^{2}}$ not fitting in with equations (200) and (199). But in practice the molecules do not move along a series of lines joined together, especially in a liquid. We may, however, (Sections 41 and 42) find a series of rectilinear paths lying close to the actual path equal to it in length, which indicate the average directions of different portions of the actual path as shown in Fig. 14, and whose lengths are grouped about a mean length according to Clausius' law, and whose directions in space are unrestricted. The parallel planes passing through the points of connection of these paths divide the actual path into a number of parts which we may take as being independent of each other as the corresponding rectilinear paths. The smallest period $t_{r}$ admissible in equations (199) and (200) would then be that corresponding to the average displacement which satisfies equation (202), where the symbol $l_{\delta r}$ now denotes the average diffusion path as defined and used in Sections 41 and 42 . Smaller values of $t_{r}$ and the corresponding values of $\overline{d^{2}}$, will therefore not satisfy equations (199) and (200).

This result may be obtained in a somewhat different way. Let us suppose as before that a molecule moves along a series of rectilinear paths. Consider the displacement corresponding to a portion of a rectilinear path for a period $t_{1}$ which is much smaller than the period of the path. The corresponding displacement $d_{1}$ is then proportional to $t_{1}$, or equal to $t_{1} C_{1}$, where $C_{1}$ is a constant. The value of $\overline{d^{2}}{ }_{r} / t_{r}$ in equation (200) under these conditions is therefore equal to $t_{r} C_{2}$, where $C_{2}$ is a constant. But this is obviously impossible, and the period $t_{r}$ can therefore be given values only equal to or greater than the period of the average path. Since the actual path of a molecule may be on the average represented by such a series of rectilinear paths, the same result holds in reference to the period of the average representative path. The deviation of the ratio from satis- 
fying equation (200) on decreasing $t_{r}$ would, however, be more gradual in this case.

If $t^{\prime}{ }_{r}$ denote the smallest admissible period that satisfies equation (200) and $\overline{d_{r}^{\prime}}$ the corresponding average of the displacements, the total average velocity $V_{t r}$ of a molecule $r$ is given by

$$
V_{t r}=\frac{l_{\delta r}}{t_{r}^{\prime}}=\frac{4 \overline{d_{r}^{\prime}}}{t_{r}^{\prime}}, \ldots . . . .
$$

by the help of equation (202); since $t^{\prime}{ }_{r}$ is then the period of the average diffusion path $l_{\delta r}$, and the representative and actual path of a molecule are equal in length (Section 41). This equation may be used to find the velocity of a colloidal particle, as will be described in Section 60 . The number of times $n_{r}$ per second that a square $\mathrm{cm}$. is crossed by molecules $r$ in one direction is given by

$$
n_{r}=\frac{N_{r} V_{t r}}{3}=\frac{4 N_{r} \overline{d_{r}^{\prime}}}{3 t_{r}^{\prime}},
$$

according to equations (203) and (35).

The foregoing remarks, and equations (203) and (204), apply also to a mixture of molecules $r$ in $e$ which is not a dilute solution.

The largeness of the value that may be taken for the period $t_{r}$ is limited somewhat by the fact that in the deduction of equation (199) it is assumed that the number of molecules undergoing a displacement $d$ at each of two equal clements of volume in a heterogeneous mixture at a distance $d$ apart is proportional to the concentration of the molecules. This will of course not hold when the difference between the concentrations in the two volumes is large. This difference evidently depends on the concentration gradient of the mixture. The admissible values of the displacement and period therefore depend on the concen- 
tration gradient, and increase with decrease of the gradient. The same remarks apply to the limits of $l_{\delta r}^{\prime}$ in Section 53.

On equating the eoefficients of diffusion given by equations (201) and (175), and eliminating $\left(l^{\prime \prime}{ }_{\delta r}\right)^{2} / t^{\prime \prime}{ }_{\delta r}$ from the resultant equation by means of equation (178), or (179), we obtain equations similar in form to the latter equations, which express $\overline{d^{2}}{ }_{r} / t_{r}$ in terms of fundamental quantities. The same remarks apply to these equations as were made in connection with equations (178) and (179).

Equation (201), which applies to a dilute solution, has previously been deduced * by Einstein by a different method, without obtaining, however, its exact limitations. The method that I have developed is more general and fundamental since it gives diffusion formula for any relative concentration of the constituents of the mixture without referring to its state, and gives the exact limitations of the formulæ. It should be noted that no restrictions whatever are made in the investigation in connection with the molecular velocities. By means of this method other diffusion formulæ, and formulæ for the viscosity and conduction of heat, can be obtained in terms of molecular displacements. These are given in subsequent Sections.

An application of the foregoing results to the Brownian motion of colloidal particles will now be given.

(a) On equating the values of the coefficients of diffusion given by equations (201) and (147) the equation

$$
\frac{\overline{R T}}{N} \dot{M}_{r}=\frac{d_{r}^{2}}{2 t_{r}}, . \quad . \quad \text {. . . . }
$$

is obtained, which expresses the coefficient of mobility of a particle, or the velocity under the action of unit force, in terms of other quantities. If the particle is spherical in shape and its size is so large that its mobility obeys Stokes'

* Ann. der Phys., 19, pp. 371-381 (1906). 
law, the coefficient of mobility given by the foregoing equation and equation (166) may be equated giving

$$
\frac{\overline{d_{r}^{2}}}{t_{r}}=\frac{R T}{N} \frac{1}{3 \pi r \eta}, \quad \text {. . . . . . . . }
$$

where $r$ denotes the radius of the particle, and $\eta$ the viscosity of the medium. This equation has been applied to the Brownian motion of colloidal particles of different substances in different solvents, the values of $d_{r}$ for a selected value of $t_{r}$ being directly observed by means of the ultramicroscope as described in Section 44, or recorded photographically by means of an appropriate arrangement. Some of the earlier observations did not fit in well with the equation. This was probably due to some extent that the period was taken below the least admissible value, the limitations of the equation not being known. Later experiments happen to have been carried out for larger periods and consequently gave a better agreement. It is also hardly likely that the colloidal particles obtained by sparking between various metal electrodes placed in a liquid should in all cases be exactly, or approximately, spherical in shape as required by the equation. We would expect that the geometrical shape of the particles should depend a good deal on the conditions of the sparking. A good confirmation of Einstein's equation was recently obtained by Nordlund,* who used particles obtained by sparking mercury in a liquid. These particles are bound to be spherical in shape since they would be in the liquid state, and hence Stokes' law would hold. Nordlund determined $N$, the number of molecules in a gram molecule, by means of the equation, and obtained the value

$$
5.91 \times 10^{23} \text {, }
$$

*Zs. f. Phy. Chem., 87, pp. 40-62 (1914). 
which is in close agreement with the values obtained by other methods.

Einstein's formula has been exhaustedly tested by Millikan* in the case of particles suspended in a gas, and found to be in complete agreement with the facts.

According to equation (201) the value of $\frac{\overline{d_{2 r}}}{t_{r}}$ is constant for different values of $d_{r}$ and $t_{r}$. This is necessary to test before using equation (206). Table XXIX shows the

TABLE XXIX

\begin{tabular}{|c|c|c|}
\hline Periods. & $z \overline{d^{2} r} \cdot$ & $z \overline{\bar{d}_{r}}$ \\
\hline $1 t$ & 1.039 & 1.039 \\
$2 t$ & 2.458 & 1.229 \\
$3 t$ & 3.957 & 1.319 \\
$4 t$ & 5.264 & 1.416 \\
$5 t$ & 6.685 & 1.337 \\
$6 t$ & 7.963 & 1.327 \\
$10 t$ & 13.528 & 1.353 \\
\hline
\end{tabular}

agreement obtained by Nordlund in the investigations just described. It will be seen that for periods of the displacement $d_{r}$ from $3 t$ to $10 t$ inclusive $Z \frac{\overline{d_{r}^{2}}}{t_{r}}$ is practically constant where $Z$ is a constant. The values for the periods $1 t$ and $2 t$ do not fit in well with the others, and they are therefore probably inadmissible values along the lines previously explained. (See also Section 60.) The result is of interest and importance since it is evidence of the soundness of the deduction of equation (199), and incidentally of the deduction of the equations in Sections 57 and 58, and of the

* The Electron, by R. A. Millikan, the University of Chicago Science Series. 
equations in Sections 53, 54, and 55, each of which involves an indeterminate linear magnitude connected with the actual path of a molecule, and its corresponding indeterminate period.

\section{5\%. The Constant Displacement Diffusion Equa-} tion.

Instead of considering the displacement of the molecules for a selected period $t$, we may consider the different periods of the molecules corresponding to a selected displacement $d$. This case may be treated along similar lines as that in the previous Section. Suppose that $n_{r_{1}}^{\prime}$ molecules $r$ per cubic $\mathrm{cm}$. have a period $t_{r_{1}}$ in undergoing a displacement $d_{r}$ in one direction at right angles to the plane of reference, and that $n_{r_{2}}^{\prime}$ molecules have a period $t_{r_{2}}$, etc. Each of these sets of molecules may be treated independently along the same lines as the investigation in the preceding Section. It follows immediately then from the previous Section that the gain per second in molecules $r$ having the period $t_{r_{1}}$ below the plane of reference is equal to

$$
\frac{n_{r_{n}}^{\prime} d_{r}^{2}}{t_{r 1} N_{r}} \frac{d N_{r}}{d x}
$$

and the gain in molecules $r$ having a period $t_{r_{2}}$ is equal to

$$
\frac{n_{r_{2}}^{\prime} d_{r}^{2}}{t_{r_{2}} N_{r}} \frac{d N_{r}}{d x}
$$

and so on. The total gain in molecules $r$ is therefore

where

$$
\left\{\frac{n_{r_{1}}^{\prime}}{t_{n_{1}}}+\frac{n_{r_{2}}^{\prime}}{t_{r_{2}}}+\ldots\right\} \frac{d_{r}^{2}}{N_{r}} \frac{d N_{r}}{d x}=\frac{\overline{t_{r}-1} d^{2}}{2} \frac{d N_{r}}{d x}
$$

$$
\overline{t_{r}-1}=\left\{\frac{2 n_{r_{1}}^{\prime}}{t_{r_{1}}}+\frac{2 n_{r_{2}}}{t_{r_{2}}}+\ldots\right\} \frac{1}{N_{r}}
$$


the mean of the reciprocals of the periods. Similarly it can be shown that the gain in molecules $e$ below the plane of reference is

$$
-\frac{\overline{t_{e}-1} d_{e}^{2}}{2} \frac{d N_{e}}{d x}
$$

where $d_{e}$ is a selected displacement and $\overline{t_{e}^{-1}}$ the mean of the reciprocals of the corresponding periods. The gain in molecules $r$ and $e$ due to their displacements is therefore

$$
\frac{\overline{t_{r}-1} d_{r}^{2}}{2} \frac{d N_{r}}{d x}-\frac{\overline{t_{e}^{-1}} d_{e}^{2}}{2} \frac{d N_{e}}{d x}
$$

But the volume of these molecules is not zero, and if this is taken into account similarly as in the previous Section we obtain that the diffusion $\delta_{r}$ of the molecules $r$ is given by

$$
\delta_{r}=\frac{1}{2} \frac{\vartheta_{e}}{N_{r} \vartheta_{r}+N_{e} \vartheta_{e}}\left\{\overline{t_{r}-1} d^{2} N_{e} \frac{d N_{r}}{d x}+\overline{t_{e}-1} d^{2} N_{r} \frac{d N_{e}}{d x} .\right\},
$$

where $\vartheta_{e}$ and $\vartheta_{r}$ denote the external molecular volumes of gram molecules of molecules $e$ and $r$ respectively in the mixture.

In the case of a dilute solution of molecules $r$ in molecules $e$ the foregoing equation becomes

$$
\delta_{r}=\frac{\overline{t_{r}-1} d^{2}}{2} \frac{d N_{r}}{d x}, \ldots . . . . .
$$

and hence the coefficient of diffusion is given by

$$
D_{r}=\frac{\overline{t_{r}^{-1}} d^{2}}{2} \text {. }
$$

It can be shown along the same lines as in the previous Section that the smallest admissible value of $d_{r}$ in equation (209) corresponds to its value in the equation

$$
4 d_{r}=l_{\delta r}, \quad . \quad . \quad \text {. . . }
$$




\section{MICSELLANEOUS APPLICATIONS, CONNECTIONS}

where $l_{\delta r}$ denotes the average diffusion path according to Section 41. If $d^{\prime}{ }_{r}$ denotes the smallest admissible value of $d_{r}$ and $\overline{t_{r}^{\prime}}$ the average of the corresponding periods, the total average velocity $V_{t r}$ of a molecule $r$ is given by

$$
V_{t_{r}}=\frac{l_{\delta r}}{\overline{t_{t}^{\prime}}}=\frac{4 d_{r}^{\prime}}{\overline{t_{r}^{\prime}}}, \ldots . . .
$$

by means of the foregoing equation, since the average diffusion path is equal to the corresponding actual path. The number of times $n_{r}$ a square $\mathrm{cm}$. is crossed per second by molecules $r$ in one direction is given by .

$$
n_{r}=\frac{N_{r} V_{t r}}{3}=\frac{4}{3} \frac{N_{r} d_{r}^{\prime}}{\overline{t_{r}^{\prime}}{ }_{r}}, \ldots . . .
$$

according to equations (211) and (35). The foregoing remarks and equations also hold when the mixture is not a dilute solution of molecules $r$ in $e$.

On equating the expressions for $D_{r}$ given by equations (209) and (147) we obtain the equation

$$
\frac{R T}{N} M_{r}=\frac{\overline{t_{r}-1} d_{r}^{2}}{2}
$$

which may be used to determine the coefficient of mobility $M_{\tau}$ of a colloidal particle. This equation and equation (166) give the equation

$$
\overline{t_{r}-1} d_{r}^{2}=\frac{R T}{N} \frac{1}{3 \pi r \eta}
$$

which depends on Stokes' law, and may be used in the same way as equation (206).

It will now be recognized that an expression for the diffusion may be obtained along the same lines corresponding to any law of selection of the displacements, or of the periods. 
58. The Constant Period and Constant Displacement Viscosity Equations.

The viscosity of a substance also may be expressed in terms of molecular displacements at right angles to a plane. Let us first obtain a formula for the viscosity in terms of displacements corresponding to a constant period.

(a) Let the plane of reference be taken parallel to the motion of the substance, and let us suppose that the velocity gradient is unity, and for convenience of reference from top to bottom. We will adopt the same notation as in Section 56 , and suppose that we are dealing with a pure substance of molecules $r$ instead of with a solution of molecules $r$ in molecules $e$. Let us suppose that a molecule at the beginning of a displacement (which takes place at right angles to the motion of the medium) abstracts the momentum $V_{1} m_{a}$ from the medium, where $V_{1}$ denotes the velocity of the medium in a plane parallel to its motion passing through the molecule whose absolute mass is $m_{a r}$. This momentum, we will suppose, is transferred to the medium at the end of the displacement. Now it follows from considerations of equilibrium that the displacement of a molecule in one direction from one plane to another, both of which are parallel to the motion of the medium, is accompanied by the displacement of another molecule from one plane to the other in the opposite direction. Therefore if $V_{1}$ and $V_{2}$ denote the velocities of the medium at two planes taken a distance $d$ from each other, the momentum transferred from one plane to the other by a molecule and its associate molecule is $\left(V_{1}-V_{2}\right) m_{a}$, or $d m_{a}$, since the velocity gradient of the medium is unity, or $\left(V_{1}-V_{2}\right) / d=1$. Since $n_{r_{1}}$ molecules of displacement $d_{\tau_{1}}$ cross the plane of reference per square $\mathrm{cm}$. in each direction during the period $t_{r}$, or $n_{r_{1}}^{\prime} d_{r_{1}}$ molecules, where $n_{r_{1}}^{\prime}$ denotes the number of molecules in a cubic $\mathrm{cm}$. undergoing a displacement $d_{r_{1}}$ in one direction, 
it follows that these molecules transfer the momentum $n_{r_{1}} d_{r_{1}}{ }^{2} m_{a r}$ aoross the plane. Similarly it follows that the molecules undergoing a displacement $d_{r_{2}}$ transfer the momentum $n_{r_{2}}^{\prime} d_{r_{2}}^{2} m_{a r}$ during the period $t_{r}$ across the plane per square $\mathrm{cm}$. and so on. The total momentum transferred per second per square $\mathrm{cm}$. of the plane, which is the coefficient of viscosity $\eta$, is therefore given by

$$
\eta=\frac{N_{r} m_{u r}}{2 t_{r}}\left\{\frac{2 n_{r_{1}}^{\prime} d_{r_{1}}^{2}+2 n_{r_{2}}^{\prime} d_{r_{2}}+\ldots}{N_{r}}\right\}
$$

or

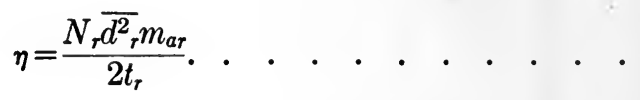

where $\overline{d^{2}}$ denotes the mean of the squares of the displacements of the molecules of a cubic $\mathrm{cm}$. during the period $t_{r}$.

It will not be difficult to see that in the case of a mixture of molecules $r$ and $e$ the coefficient of viscosity is given by the equation

$$
\eta=\frac{N_{r} \overline{d^{2}}{ }_{r} m_{a r}}{2 t_{r}}+\frac{N_{e} \overline{d^{2}}{ }_{\epsilon} m_{a e}}{2 t_{e}} . . . .
$$

where $\overline{d^{2}}{ }_{e}$ denotes the mean of the squares of the displacements of the molecules $e$ in a cubic $\mathrm{cm}$. during the period $t_{e}$.

It can be shown in a similar way as in Section 56 in connection with diffusion that the smallest admissible value of $d^{2}{ }_{r}$ in equations (215) and (216) corresponds to the value of the mean of the displacements $\bar{d}_{r}$ which satisfies the equation.

$$
4 \bar{d}_{r}=l_{\eta r}, \quad . \quad . \quad . \quad . \quad \text {. }
$$

where $l_{\eta r}$ denotes the mean momentum transfer distance of a molecule $r$ as defined in Section 33 . 
If $\overline{d^{\prime}}$ denotes the smallest admissible mean of the displacements and $t^{\prime}{ }_{r}$ the corresponding period, the total average velocity is given by

$$
V_{t_{r}}=\frac{l_{l_{r}}}{t_{r}^{\prime}}=\frac{4 \overline{d_{r}^{\prime}}}{t_{r}^{\prime}}, \quad \ldots . . .
$$

while the number of molecules $n_{r}$ crossing a square $\mathrm{cm}$. in one direction is given by

$$
n_{r}=\frac{N_{r} V_{t r}}{3}=\frac{4}{3} \frac{N_{r} \overline{d^{\prime \prime}} r}{t_{r}^{\prime}}, \quad . . . .
$$

obtained along the same lines as similar equations in Section 56.

If equation (201) is supposed to apply to the diffusion of a molecule in a substance of the same kind and the period is taken the same as the period in equation (215) and eliminated from the equations we obtain the equation

$$
\frac{\overline{d^{2}{ }_{\eta r}}}{\overline{d^{2}{ }_{\delta r}}}=\frac{\eta}{D_{r} N_{r} m_{a r}}, \quad . \quad . \quad . \quad . \quad .
$$

where the suffixes $\delta$ and $\eta$ have been added to the displacements to indicate what each refers to. According to this equation the ratio on the left-hand side is not in general equal to unity. Thus the displacement of a molecule along an axis is affected by a shearing motion of the substance at right angles to the axis.

In the case of a dilute solution of molecules $e$ in $r$ the value of $\overline{d^{2}}{ }_{r}$ will very approximately be the same as for the solvent in the pure state, and may accordingly be obtained for any selected period $t_{r}$ from equation (215). The value of $\overline{d^{2}}{ }_{e}$ may then be obtained for any selected period $t_{e}$ from equation (216). It would furnish interesting information to compare this value of $\overline{d^{2}}{ }_{e}$ with that of a pure substance 


\section{MISCELLANEOUS APPLICATIONS, CONNECTIONS}

of molecules $e$, or if the solute consists of colloidal particles, to compare the value with that observed.

If $\eta$ is eliminated from equation (215) by means of one of the equations (90), (91), and (92), an equation is obtained which expresses the ratio $\overline{d^{2}} / t_{r}$ in terms of more fundamental quantities, the equation being similar to one of the equations (188), (189), and (190).

If we write $t_{e}=t_{r}$ and $\overline{d^{2}{ }_{e}}=\overline{d^{2}}$ in equation (216) it gives for any selected value of $t_{r}$ a value of $\overline{d^{2}}{ }_{r}$ which in a sense is a mean value of $\overline{d^{2}}$ and $\overline{d^{2}}$. It would be interesting to compare this value with that of the substance $r$ in the pure state.

The values of $\overline{d^{2}}$ and $\overline{d^{2}}$ in equation (216) may be determined for any selected values of $t_{r}$ and $t_{e}$ by equating each of the two terms on the right-hand side of the equation with the corresponding term on the right-hand side of equation (98), and calculating the other quantities involved in the way described in Section 35.

It can easily be proved along the same lines as the preceding investigation that if the periods of the molecules in a cubic $\mathrm{cm}$. for a selected displacement $d_{r}$ are observed, the coefficient of viscosity is given by

$$
\eta=? N_{r} d_{r}^{2} \overline{t_{r}-1} m_{a r}, \ldots . . . .
$$

where $\overline{t_{r}-1}$ denotes the mean of the reciprocals of the periods corresponding to the displacement $d_{r}$ and is given by

$$
\overline{t_{r}-1}=\left\{\frac{2 n_{r_{1}}^{\prime}}{t_{r_{1}}}+\frac{2 n_{r_{2}}^{\prime}}{t_{r_{2}}}+\ldots\right\} \frac{1}{\left(2 n_{r_{1}}^{\prime}+2 n_{r_{2}}^{\prime}+\ldots\right)}
$$

In the case of a mixture of molecules $r$ and $e$ we have

$$
\eta=\frac{1}{2} N_{r} d^{2}{ }_{r} \overline{t_{r}^{-1}} m_{a r}+\frac{1}{2} N_{e} d^{2} \bar{t}_{e^{-1}} m_{a e} . \quad .
$$

The smallest admissible value of $d_{r}$ in equation satisfies the equation

$$
4 d_{r}=l_{\eta r}, \quad . \quad . \quad . \quad . \quad \text {. }
$$


which follows similarly as before. The formulæ for the total average velocity and the number of molecules $r$ crossing per second a square $\mathrm{cm}$. from one side to the other in terms of the smallest admissible displacement $d^{\prime}{ }_{r}$ and the corresponding mean of the periods $\overline{t_{r}^{\prime}}$ are the same in form as equations (218) and (219). Similar relations apply in the case of the molecules in a mixture of molecules $r$ and $e$.

\section{The Constant Period and Constant Dis-} placement Heat Conduction Equations.

We will now find an expression for the coefficient of conduction of heat in terms of molecular displacements, using the same notation as before, and taking the plane of reference at right angles to the flow of heat, which we will suppose takes place from top to bottom. A formula involving the displacements for a selected constant period will be first obtained. Let us suppose that a molecule $r$ at the beginning of a displacement, (which takes place parallel to the flow of heat at right angles to the plane of reference) abstracts the energy $T_{1} S_{m r}$ from the medium and transfers it to the medium at the end of the displacement, where $T_{1}$ denotes the absolute temperature of the medium at the beginning of the displacement, and $S_{m r}$ the corresponding internal specific heat at constant pressure per molecule. Since no matter is transferred from one plane to another, each of which is at right angles to the flow of heat, it follows that the displacement of the foregoing molecule is accompanied by the displacement of another molecule in the opposite direction, both displacements lying between the same two parallel planes. Therefore if $T_{1}$ and $T_{2}$ are the absolute temperatures in descending order of magnitude of the medium in these planes the energy $T_{1} S_{m r}-T_{2} S_{m r}$ is transferred to the lower plane by a pair of corresponding molecules during the period $t_{r}$. If the distance between 


\section{MISCELLANEOUS APPLICATIONS, CONNECTIONS}

the planes is $d$ and the heat gradient is equal to unity $T_{1}-T_{2} / d=1$, and the amount of heat transferred may be written $d S_{m r}$. Since $n_{r_{1}}$ molecules of displacement $d_{r_{1}}$ cross the plane of reference in both directions during the period $t_{r}$, which number (Section 56) is equal to $n_{r_{1}}^{\prime} d_{r_{1}}$, it follows that these molecules transfer the energy $n_{r_{1}}^{\prime} d_{r_{1}} S_{\text {wrr }}$ per square $\mathrm{cm}$. across the plane. Similarly it follows that the molecules of displacement $d_{2 r}$ transfer the energy $n_{r_{2}}^{\prime} d_{r_{2}}{ }^{2} S_{m_{\tau}}$ per square $\mathrm{cm}$. across the plane, and so on. The total energy transferred per square $\mathrm{cm}$. per second across the plane, or the heat conductivity $C$, is therefore given by

$$
C=\frac{N_{\tau} S_{m}}{2 t_{r}}\left\{\frac{2 n_{r_{1}}^{\prime} d_{r_{1}}^{2}+2 n_{r_{2}}^{\prime} d_{r_{2}}^{2}+\ldots}{N_{r}}\right\}
$$

or

$$
C=\frac{N_{r} S_{m r}}{2} \frac{\overline{d^{2}}}{t_{r}}, \quad . \quad . \quad . \quad . \quad . \quad . \quad . \quad .
$$

where $\overline{d^{2}}{ }_{r}$ denotes the mean of the squares of the displacements of the molecules in a cubic cm. If $S_{g r}$ denote the internal specific heat at constant pressure per gram we have $S_{m r}=S_{\text { }} m_{a r}$, where $m_{a r}$ denotes the absolute molecular weight of a molecule $r$, and the foregoing equation may therefore be written

$$
C=\frac{N_{r} m_{a r} S_{g r}}{2} \frac{\overline{d_{r}^{2}}}{t_{r}} \cdot \ldots . . .
$$

In the case of a mixture of molecules $r$ and $e$ we have

$$
C=\frac{N_{r} m_{a r} S_{g r}}{2} \frac{\overline{d^{2}}}{t_{r}}+\frac{N_{e} m_{a e} S_{a e}}{2} \frac{\overline{d_{e}^{2}}}{t_{e}} . . .
$$

It can similarly be shown that if the displacement $d_{r}$ is kept constant we have

$$
C=\frac{N_{r} m_{a r} S_{o r}}{2} d_{r}^{2} \overline{t_{e}-1}, \ldots . . . . . .
$$


and

$$
C=\frac{N_{r} m_{a r} S_{o r}}{2} d^{2}{ }_{r} \overline{t_{r}-1}+\frac{N_{e} m_{a e} S_{o e}}{2} d^{2}{ }_{e} \overline{t_{e}-1}
$$

where $\overline{t^{-1}}$ denotes the mean of the reciprocals of the periods of the molecules in a cubic $\mathrm{cm}$. corresponding to the displacement $d_{r_{1}}$ and $\overline{t_{e}^{-1}}$ has a similar meaning.

The least admissible value of the average displacement $d_{r}$ in the foregoing equations can be shown, similarly as in the preceding Sections, to be the value which satisfies the equation

$$
4 d_{r}=l_{c r}
$$

where $l_{c r}$ denotes the heat transfer distance as defined in Section 37. The magnitude that may be given to the value of $d_{r}$ is limited somewhat by the fact that in the deduction of the equations we have assumed that the specific heat along the heat gradient at two points is independent of their distance apart. But this cannot hold unless the heat gradient is taken infinitely small. The formulæ for the heat conductivity may be given forms taking the variation of $S_{m r}$ and $N_{r}$ along the heat gradient into account, but on account of their complexity they possess no particular interest or importance.

If the periods are taken the same in equations (225) and (215) and eliminated we obtain the equation

$$
\frac{\overline{d^{2}{ }^{2}}}{\overline{d^{2}{ }_{\eta r}}}=\frac{C}{\eta S_{g r}}, . . . . . \cdot
$$

where the suffixes $c$ and $\eta$ have been added to the displacements to indicate to what they refer. The right-hand side of the equation is evidently in general not equal to unity, and hence the nature of the motion of a molecule is influenced in different ways by a heat gradient and a shearing motion in a substance. It should be noted that $\overline{d^{2}{ }_{c r}}$ 
refers to unit heat gradient, and $\overline{d^{2}{ }_{\eta}}$ to unit velocity gradient of the substance. According to Section 38 the right-hand side of the foregoing equation is greater than unity when the substance is in the gaseous state, and smaller than unity when in the liquid state. Thus the displacement of a molecule for a given period in a heat gradient is relatively greater than in a velocity gradient of a substance. when it is gaseous, while the opposite holds when it is in the liquid state. This appears to indicate that the path of a particle in a gas is rendered relatively more undulatory in character by a velocity gradient than by a heat gradient, while the opposite holds in the case of a liquid.

The displacements and periods may, be expressed in terms of more fundamental quantities similarly as in the previous Section. The results may be used to find approximate values of the former quantities in the case of a mixture by the help of Section 38 .

60. Another Method of Determining the Total Average Velocity of Translation of a Colloidal Particle.

We have seen in Section 56 that equation (201) holds only when the period $t_{r}$ is equal to, or larger, than that corresponding to the average displacement which satisfies equation (202). The smallest value of $t_{r}$ admissible may be determined by using successively values of $t_{r}$ in decreasing order of magnitude and observing the corresponding values of the displacements, till values are obtained which do not satisfy equation (201), or equation (206) if Stokes' law holds. The value of $t_{r}$ corresponding to the transition point is the smallest admissible value. On substituting this value and the value of the corresponding average displacement in equation (203) the value obtained for $V_{t r}$ is the total average velocity of the particle. 
Since $d_{r}$ on the average is proportional to $t_{r}$ when its value is inadmissible according to the Section cited, it follows from equations (201) and (206) that in that case

$$
\overline{d_{r}^{2}} t_{r} D_{r} \quad . \quad . \quad . \quad . \quad . \quad . \quad . .
$$

and

$$
\overline{\frac{d^{2}}{t_{r}}}<\frac{R T}{N} \frac{1}{3 \pi r \eta} . \quad . \quad . \quad . \quad . \quad . \quad .
$$

Thus as the value of the period is decreased a point is ultimately reached when the ratio $\overline{d^{2}}{ }_{r} / t_{r}$ ceases to be constant, and decreases proportionally to $t_{r}$ for further decreases in $t_{r}$. Therefore on plotting $\overline{d_{r}^{2}} / t_{r}$ against $t_{r}$, and drawing a mean straight line through the points which indicate that the ratio is constant, and a mean straight line through the other points, the intersection of the two lines gives the period $t^{\prime}{ }_{r}$ and displacement $d^{\prime}{ }_{r}$ which on being substituted in equation (203) give the total average velocity of the colloidal particle.

No experiments have of course yet been carried out with the object of determining the total average velocity of a colloidal particle in this way. It seems probable, however, that Nordlund in testing the constancy of $\overline{d_{r}^{2}} / t_{r}$ in the experiments described in Section 56 advanced into a region in which the values of $\overline{d^{2}}{ }_{r}$ and $t_{r}$ are inadmissible in equation (206). Thus an inspection of Table XXIX shows that the ratio in one set of experiments, though constant for periods of $3 t$ and $10 t$ inclusive where $t=1.481$ sec., decreases considerably for smaller periods. If this deviation is genuine, as it seems to be, it will be possible to determine from Nordlund's experiments whether the velocity of a colloidal particle is the same as if it were in the perfectly gaseous state. The radius of the mercury particle corresponding to the values in the Table cited was $2.66 \times 10^{-5} \mathrm{~cm}$. The 
velocity that it would have if it behaved as if it were in the perfectly gaseous state is $3.33 \times 10^{-1} \mathrm{~cm}$. $/ \mathrm{sec}$. The average value of $\overline{d^{2}}$ obtained by experiment corresponding to periods which were multiples of 1.481 sec. was $1.813 \times 10^{-8}$ $\mathrm{cm}$., and $t_{r}$ is therefore equal to $1.481 \mathrm{sec}$. The average displacement is probably very approximately given by the square root of $\overline{d^{2}}{ }_{r}$, and hence the apparent velocity of the particle according to equation (203) is

$$
3.64 \times 10^{-4} \mathrm{~cm} . / \mathrm{sec} \text {. }
$$

If this represents approximately the total average velocity of the particle, as is rendered probable on account of the deviations mentioned, it follows that this velocity is about $1 / 1000$ of that the particle would have in the perfectly gaseous state. This result fits in with those of Section 49 .

Some experiments by Henri* should be mentioned in this connection. He obtained cinematograph photographs of the Brownian motion of colloidal particles of rubber of $1 \mu$ radius in water. The displacements observed, which corresponded to a period of $\frac{1}{20}$ of a second, were four times smaller than those calculated from Einstein's equation. This deviation might have been caused by the period being smaller than the least admissible value of the period, since the deviations are in the right direction. It is true that Henri tested whether $\overline{d^{2}} / t_{r}$ is constant, though this point was not specially investigated, and found the ratio approximately constant. But the deviation of this ratio from a constant when it occurs is probably very gradual, and may therefore be approximately constant in the inadmissible region for values lying between limits of considerable magnitude. It is difficult to see how in Henri's experiments an error could have come in to produce a deviation of the magnitude mentioned.

* Compt. Rend., 146, 1024-1026, (1908). 
It is highly desirable that experiments be carried out

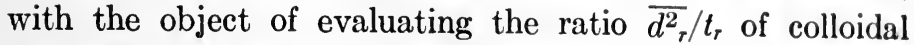
particles for as small periods as possible in order to obtain in one or more cases with certainty the smallest period that will satisfy equation (206), and to calculate from that the total average velocity of the particle concerned. It is probably not accidental that in all cases that have come under my notice the above ratio when not fitting in with equation (206) fitted in with the inequality $(A)$ which corresponds to periods not admissible in equation (206).

\section{The Distribution of the Molecular Velocities} in a Substance not Obeying the Gas Laws.

It does not seem possible as yet to obtain any definite theoretical information about this distribution. The provf of Maxwell's law given in Section 7 applies only to gases, and the law need not therefore hold for a substance not obeying the gas laws. It is very probable, however, that the distribution of molecular velocities obeys Maxwell's law in all cases. This may be tested directly for colloidal particles in solution. Thus according to Section 7 the probability that the velocity of a molecule has values lying between $V_{1}$ and $V_{1}+d V_{1}$ and is

$$
\frac{4 V_{1}^{2}}{V_{D}^{3} \sqrt{\pi}} e^{-\frac{V_{1}^{2}}{V_{\nu}^{2}}} \cdot d V_{1} .
$$

This expression may be converted into one involving displacements (Section 56) as follows: The projection of the path of a particle between two points on to an axis is the same as if the points were joined by a straight line. Now if the path of each particle were divided into parts corresponding to the same period $t_{t}$, these joined by straight lines, and the particle supposed to move along them, the law 
of distribution of velocities would probably not be altered if $t_{r}$ is below a certain limit, where $V_{1}$ and $V_{p}$ would now refer to the new conditions. Instead of directly transforming the foregoing expression into one involving displacements, it will be more convenient to derive the required expression from one used in the deduction of the foregoing probability expression. Thus we have seen in Section 7 that the probability that a molecule has a component velocity lying between $a$ and $a+d a$ is

$$
\frac{1}{V_{p} \sqrt{\pi}} e^{-\frac{a^{2}}{V_{p^{2}}} \cdot d a}
$$

The quantity $V_{p}$ in the expression may be expressed in terms of the average $\bar{d}_{r}$ of the displacements corresponding to the period $t_{r}$. According to Section 7 we have

$$
V_{t}=2 \frac{V_{p}}{\sqrt{\pi}}
$$

where $V_{t}$, the total average velocity of a molecule in any state, now takes the place of $V_{a}$, the average velocity in the gaseous state. On applying equation $2 p=l_{\delta}$ in Section 44 to this case by considering the different molecular velocities individually and adding up the results, we obtain

$$
V_{t}=4 \overline{d_{r}} / t_{r} .
$$

The foregoing two equations give the equation $V_{p}=2 \sqrt{\pi} \bar{d}_{r} / t_{r}$, which expresses $V_{p}$ in terms of $\bar{d}_{r}$. Therefore on substituting this expression for $V_{p}$, and writing $\overline{d_{r}} / t_{r}$ for $a$, in the foregoing probability expression, and considering a number of displacements $N_{d}$, we obtain

$$
\frac{N_{d}}{\bar{d}_{r} \sqrt{\pi}} e^{\frac{d_{r}^{2}}{4 \pi\left(\overline{d_{r}}\right)^{2}}} \cdot d\left(d_{r}\right)
$$


for the number of the displacements $N_{d}$ which have values lying between $d_{r}$ and $d+d\left(d_{r}\right)$ corresponding to the period $t_{r}$. The values of the observed displacements appear to agree with the foregoing law of distribution, and Maxwell's law for the distribution of molecular velocities holds therefore, at least approximately for colloidal particles.

The most probable displacement, which is obtained by dividing the foregoing expression by $N_{d}$, differentiating it with respect to $d_{r}$, and equating the result to zero, is equal to zero. The reason for this is not difficult to see. Consider the molecular paths of the same length per period $t_{r}$ passing in all directions through any given point. It is evident then that the number of molecules associated with a given projection increases with a decrease in its value. It is of importance to point out that the average displacement obtained by direct observation will therefore tend to be rather too large than too small. The total average velocity of a colloidal deduced according to Section 56 will therefore tend to be too large rather than too small, a point which is of importance in determining whether this velocity is the same as that corresponding to the gaseous state.

The value of the most probable velocity $V_{p}$ in Maxwell's distribution law will depend, according to what has gone before, on the solvent in which the particles are suspended besides on their mass, and in the case of a molecule the value of $V_{p}$ will increase with increase of density of the substance at constant temperature, since we have seen that its total average velocity increases with the density. 



\section{SUBJECT INDEX}

Absolute temperature: Deduction of, 12; correction of scale of, 14; zero of, 14.

Amplitude: Of motion of colloidal particle, 9, 189, 190.

Atom: Absolute mass of hydrogen, 7.

Attraction: Forces of, surrounding molecules and atoms, 46, 47.

Brownian motion: Of particles in liquids, 9, 189, 190, 217-219, 250, 251 ; of particles in gases, $10,251$.

Calorie: Definition of, 38 .

Colloidal particles: Preparation of, 10.

Conduction of heat: Coefficient of, 148; characteristic function of, $154,159,161$; displacement formulæ for, 259-262; extended forms of formulæ for, 238-241; formula for, of pure gas, 157; formula for, of gaseous mixture, 165; general formulæ for, of pure substance, 155; general formulæ for, of mixtures, 164; interference function of, 154,158,162,163; mean transfer distance in, 148150 ; nature of 147; of gaseous mixtures, 165; of liquids, 162, 163; of pure gases, 158, 159; of solids, 166, 167, 184-186.

Crookes' radiometer: Action of, 10.

Diffusion: Cause of, 168; characteristic function of, 174, 177, 179181 ; coefficient of, 169; connection of coefficient of, with mobility, 198; displacement formulæ for, 242-249, 252-254; formula for, of gases, 179; general formula for, 173; interference function of, 174, 175, 178; Maxwell's expression for, of gases, 187; mean path of colloidal particle, 192, 193; tables of various coefficients of, 180, 199.

Displacement and its period: Constancy of ratio of square of first to second, 251; table of values of, 251.

Effect: Joule-Thomson, 46, 47.

Electric conductivity: Formulæ for, 182-186; ratio of, to heat conductivity, 184-186. 
Electrons: Charge carried by 7 ; concentration of, in metals, 182, 183; dissociation of atoms into, 166; number of, crossing a square $\mathrm{cm}$. of a substance per second, 167; variation of concentration of, with change of temperature, 183.

Energy: Equipartition of molecular kinetic, in gaseous mixture, 31; internal molecular, 41, 103; kinetic, per molecule of a gas, 29; potential, of attraction, 103; total internal, of a substance, 103.

Factor: For converting calories into ergs, 38 .

Gases: Equation of pure, 12; equation of mixture of, 13; equation of, from dynamical considerations, 29, 30; value of $R$ in equation of, 14 .

Heat: Latent of evaporation, 46, 205.

Heat gradient: Effect of, on molecular path, 240, 241.

Interaction: Of molecules and atoms, 48-50.

Intrinsic pressure: Cause of 69 ; comparison with other quantities, 73; formulæ for, 70, 71, 104; from equation of state, 84; partial intrinsic, 202-206; tables of values of, 72, 139.

Linear diameter law, 79.

Matter: Atomic nature of, 3-5.

Maxwell's law of distribution of molecular velocities: Consideration of, 20-27.

Molecules: Formulx for number of, per c.c. in a gas, 33, 34; nature of motion of, 225, 226; number of, per gram molecule, from charge on the electron and electrolysis, 7; from observed displacements and Stokes' law, 250; from counting and Van't Hoff's law, 217-219.

Mobility: Connection of coefficient of, with osmotic pressure, 197; coefficient of, expressed in terms of molecular displacements, 249254; of a colloidal particle in an electric field, 200, 201; of a colloidal particle in terms of its path and period, 199, 200; table of coefficients of, 199.

Number of molecules $n$ crossing a square $\mathrm{cm}$. in one direction: Determination of, by an equation of equilibrium, 95-105; formula for, corresponding to any state, 56; in a gas, 34-37; inferior and superior limits of, 74, 75; inferior limit, of 80 ; probability series for, 96 . 
Osmotic pressure: Cause of, in heterogeneous mixtures, 208, 209; connection of, with molecular motion in a dilute solution, 213-217; constants of, in heterogeneous dilute solution, 217; in connection with diffusion, 197; permanent nature of, 21 .

Path satisfying given conditions: Mean and mean of squares of, 109; probability of, lying between given limits, 107; probability of, cutting a plane, 108.

Polymerization: Of mercury, 105.

Pressure: External, given by equation of state, 81-86.

Pressure of expansion: Average, per single molecule in any state, $35-37$; nature of, in a substance in any state, $62-64$; of mixtures, 67,68 ; total, in terms of other quantities, 65,66 .

Repulsion: Forces of, surrounding atoms and molecules, 47.

Shearing motion: In viscosity, 110, 111; effect of, on path of particle, 232.

Sound: Formula for velocity of, 41.

Specific heats: Of gases, 39, 40; of liquids and dense gases, 42-44, 103,104 ; ratio of the two, of gases, $40,41$.

State, corresponding: Nature of, 91-94.

State, equations of: Conditions that they have to satisfy, at critical point, 88 ; at absolute zero, 89 ; thermodynamical, 90,91 ; various forms, 81-86.

Stokes' law: Formulæ involving, 220, 250; nature of, 220.

Thermodynamics: First law of, 37, 38.

Volume, external molecular: Definition of, 172, 173.

Volume, internal molecular, or $b$ : Cause of, 78, 79; determination of, 95-105; mathematical definition of, 58; properties of, 58, 59, 61; superior limit of, 78, 79 .

Volume, real and apparent: Discussion of, 77, 78.

Velocity of a molecule: Average kinetic energy, in a gas, 28; average, in a gas, 20; determination of total average, 95-103, 139; inferior limit of total average, 80 ; most probable, in a gas, 20; variation of, with time in a gas, 20; variation of, with time in a substance in any state, 265-267; when not under the action of a force in a gas or liquid, 52,53 .

Velocity of colloidal particle: Determination of, 195, 210, 262-265. 
Viscosity: Cause of, 110, 111; characteristic function of, 121, 128, $129,130,144,145$; definition of coefficient of, 111; definition of momentum transfer distance of, 113-116; displacement formula for, 255-258; extended formulæ for, 230-238; formula for, of a gas, 125, 126, 131; general formulx for, 122, 142, 143; interference function of, $121,131,138,140,141,145-147$; measurement of coefficient of, 112; various factors influencing it, 135-136. 




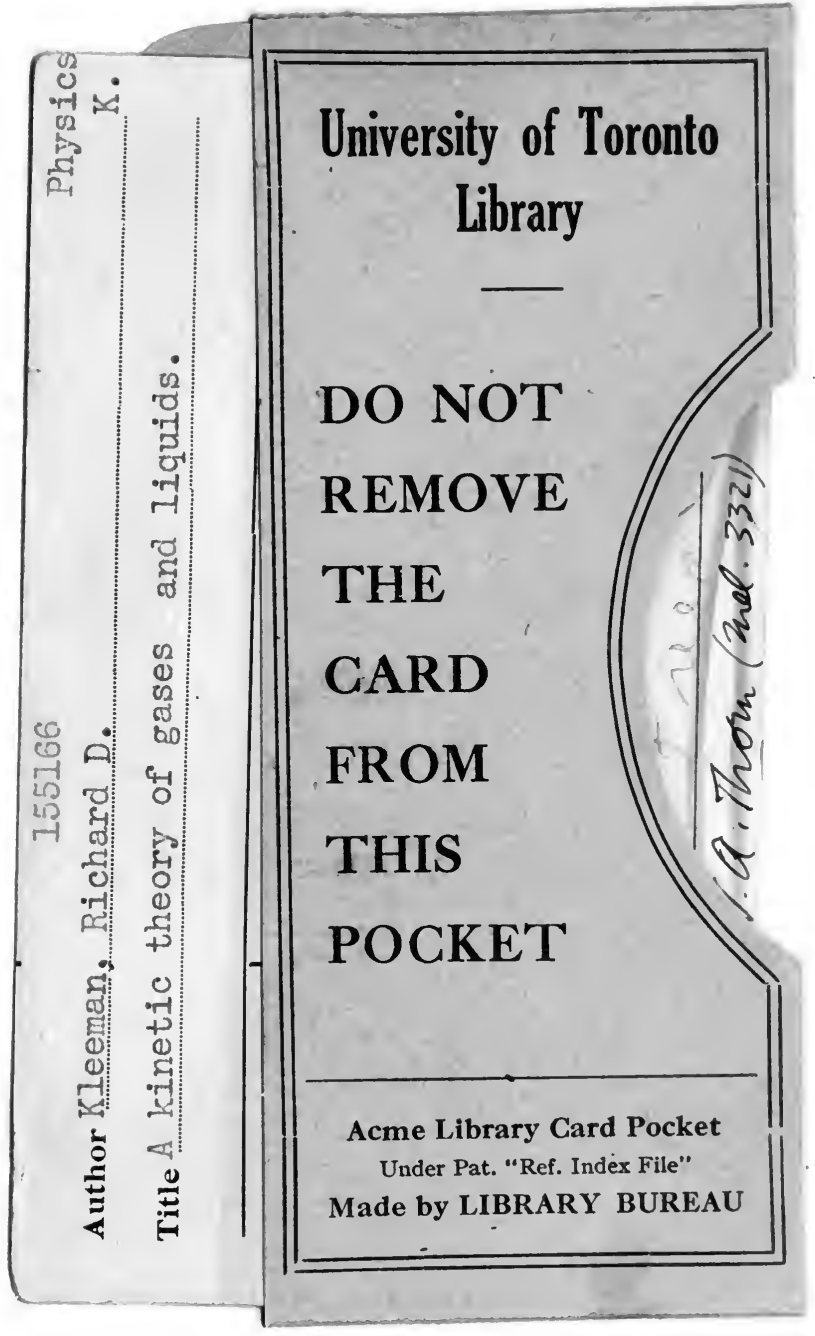


